\title{
NOVEL ECONOMICAL EMISSION MONITORING TECHNOLOGY FOR \\ LIQUID STORAGE TANKS (LST)
}

by

\section{Clifton Pereira}

A thesis submitted to The Faculty of Graduate Studies and Research

in partial fulfilment of the degree requirements of

Master of Applied Science in Mechanical Engineering

Ottawa-Carleton Institute for

Mechanical and Aerospace Engineering

Department of Mechanical and Aerospace Engineering

Carleton University

Ottawa, Ontario, Canada

July 2012

Copyright $@ 2012$ - Clifton Pereira 
Library and Archives

Canada

Published Heritage

Branch

395 Wellington Street

Ottawa ON K1A ON4

Canada
Bibliothèque et

Archives Canada

Direction du

Patrimoine de l'édition

395 , rue Wellington

Ottawa ON K1A ON4

Canada
Your file Votre référence

ISBN: $978-0-494-93511-8$

Our file Notre référence

ISBN: $978-0-494-93511-8$
NOTICE:

The author has granted a nonexclusive license allowing Library and Archives Canada to reproduce, publish, archive, preserve, conserve, communicate to the public by telecommunication or on the Internet, loan, distrbute and sell theses worldwide, for commercial or noncommercial purposes, in microform, paper, electronic and/or any other formats.

The author retains copyright ownership and moral rights in this thesis. Neither the thesis nor substantial extracts from it may be printed or otherwise reproduced without the author's permission.
AVIS:

L'auteur a accordé une licence non exclusive permettant à la Bibliothèque et Archives Canada de reproduire, publier, archiver, sauvegarder, conserver, transmettre au public par télécommunication ou par l'Internet, prêter, distribuer et vendre des thèses partout dans le monde, à des fins commerciales ou autres, sur support microforme, papier, électronique et/ou autres formats.

L'auteur conserve la propriété du droit d'auteur et des droits moraux qui protege cette thèse. $\mathrm{Ni}$ la thèse ni des extraits substantiels de celle-ci ne doivent être imprimés ou autrement reproduits sans son autorisation.
In compliance with the Canadian Privacy Act some supporting forms may have been removed from this thesis.

While these forms may be included in the document page count, their removal does not represent any loss of content from the thesis.
Conformément à la loi canadienne sur la protection de la vie privée, quelques formulaires secondaires ont été enlevés de cette thèse.

Bien que ces formulaires aient inclus dans la pagination, il n'y aura aucun contenu manquant. 
The undersigned recommend to

the Faculty of Graduate Studies and Research

acceptance of the thesis

NOVEL ECONOMICAL EMISSION MONITORING TECHNOLOGY FOR

LIQUID STORAGE TANKS (LST)

\author{
Submitted by Clifton Pereira \\ in partial fulfilment of the requirements for the degree of \\ Master of Applied Science in Mechanical Engineering
}

Dr. Matthew Johnson, Thesis Supervisor

Dr. Metin Yaras, Chair, Department of Mechanical and Aerospace Engineering

Carleton University

2012

ii 


\section{Abstract}

A low-cost Mid Infrared (IR) direct absorption spectroscopic cross correlation velocimetry (DAS-CCV) technique for hydrocarbon line flux measurements has been developed and tested experimentally. This research was conducted as the proof-ofconcept stage in the development of an economical sensor system to quantify volatile organic compound (VOC) emissions from liquid storage tanks (LST). A Mid-IR $(3.392 \mu \mathrm{m})$ Helium Neon laser was used to create two vertically displaced laser lines that were used to measure instantaneous, path integrated concentrations through a buoyant hydrocarbon (methane) plume. From these measurements, the fluctuating component of the concentration signals, corresponding to the eddy flow within the plume, were compared using a statistical technique known as $\mathrm{CCV}$ to estimate the velocity of the hydrocarbon plume. From experiments using a flow-through spectroscopic cell (with a controlled path length) filled with methane diluted in air, the sensor was able to achieve accurate measurements of $300-900 \mathrm{ppm} \cdot \mathrm{m}$ at a maximum uncertainty of $1.35 \%$. The analysis of the noise performance of the in-lab experimental apparatus demonstrated a theoretical sensor detectivity limit of 2.22 parts per million-meter $(\mathrm{ppm} \cdot \mathrm{m})$. DAS-CCV experiments were conducted using both air-methane and helium-methane mixtures. Results showed that line flux measurements were achieved with uncertainties of $2-7.5 \%$ (air- $\mathrm{CH}_{4}$ ) and 5-19\% (helium- $\mathrm{CH}_{4}$ ). From Schlieren imaging, it was determined that the higher uncertainties were attributable to the $39.1 \mathrm{~mm}$ beam spacing which allowed for noticeable diffusion of the eddies (particularly for the helium mixtures) as they traversed the two beams. Overall, the proof-of-concept system demonstrated that the DAS-CCV technique is a viable approach and the traverse tests demonstrated the potential of a full scale multiline, gridded sensor to measure the emissions released by LSTs with good accuracy and for a relatively low monetary investment. 


\section{Acknowledgements}

This thesis would not have been possible without the support of my supervisor, Dr. Matthew Johnson from Carleton University. His encouragement and patience has kept me going throughout this journey and I would like to formally thank him for this research opportunity, where I gained skills and experience that will be invaluable throughout my career.

I would like to thank my fellow co-workers of Dr. Johnson's research group that have helped me with my research and made the lab environment bearable. I would like to specifically thank, in no particular order, Graham Ballachey, Stephen Schoonbaert, Brian Crosland, David Tyner, Jan Gorski, Ian Joynes, Carol Bereton, and Darcy Corbin. I would also like to thank the members of the $\mathrm{ABL}$ lab, for being my second home away home, for cheering me up during the darker days, lending me an idea or two when I had hit a wall and celebrating with me during the lighter days. I would specifically like to thank, in no particular order, Kyle Klumper, Kyle Chisholm, Aliasgar Morbi, Ahmed Sabry, Richard Berenek, Angela Schuegraf, Jennifer Baba and Dr. Ahmadi.

I would like to thank the staff of the department of mechanical and aerospace engineering, specifically, Marlene Groves, Christie Egbert, Nancy Powell, Neil McFadyen, Bruce Johnston, Alex Proctor and Kevin Sangster for all their help and their boundless patience during my graduate career.

I would also like to thank my friends in Ottawa and Toronto, who kept me sane throughout my graduate studies and reminded me there was a life outside of the lab. I would like to specifically thank, Omer Lari, Xiong Mai, Natsuki Okabe, Kanako Oshima,

John Dahms, Peter Berg, Tina Su, Becky Yuen and Michael Troung. Finally, I would like to thank my parents and my sisters, for their understanding and support throughout my graduate studies. 


\section{Table of Contents}

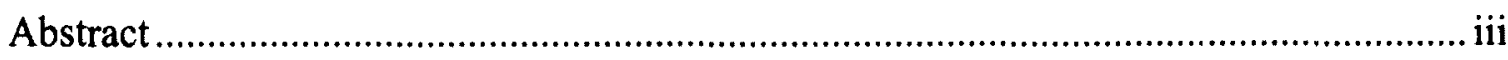

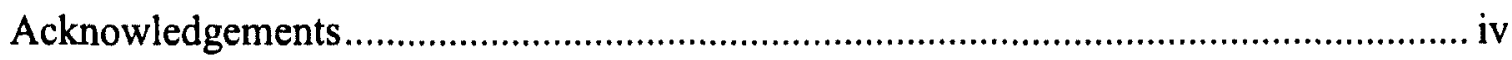

List of Tables ...................................................................................................... ix

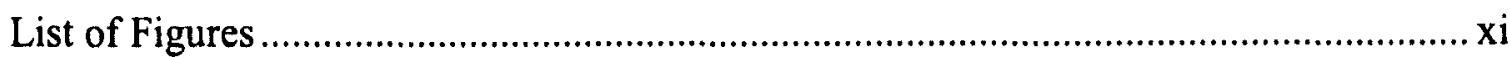

Nomenclature ............................................................................................ Xvii

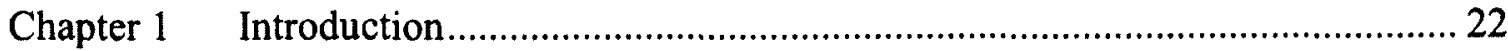

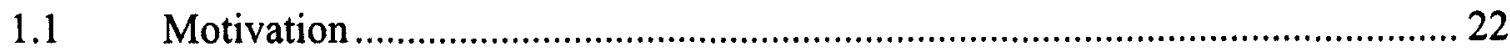

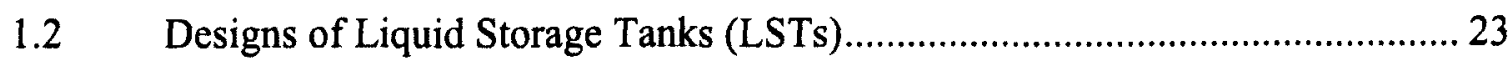

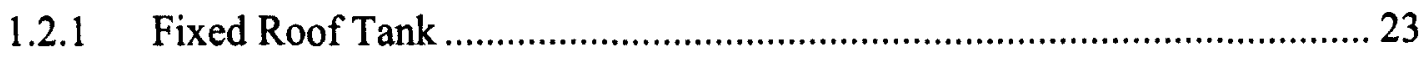

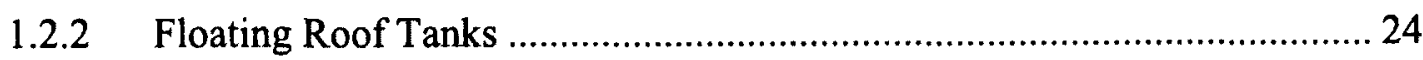

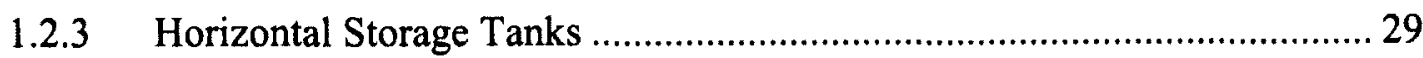

1.3 Previous Comparisons of Estimated Emissions from Liquid Storage Tanks with

Field Measurements using Differential Absorption LIDAR (DIAL) ............................ 30

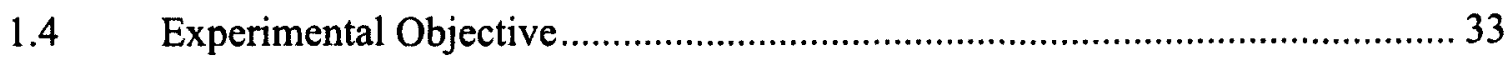

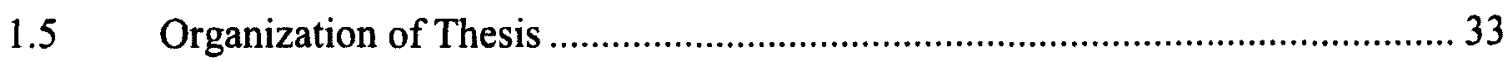

Chapter 2 Remote Sensing of Evaporative Losses from Liquid Storage Tanks ......... 35

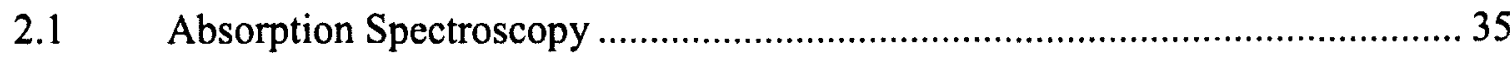

2.1.1 Direct Absorption Spectroscopy (DAS) …....................................... 36

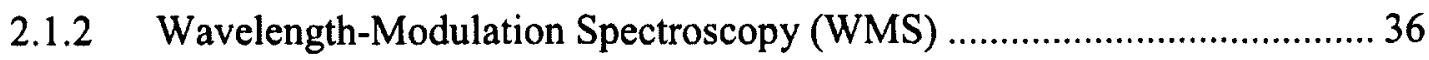

2.1.3 Differential Absorption Light Detection and Ranging (DIAL) .................. 37

2.1.4 Solar Occultation Flux (SOF) Method............................................... 38

2.2 Velocity Measurement Techniques using Absorption Spectroscopy............... 39

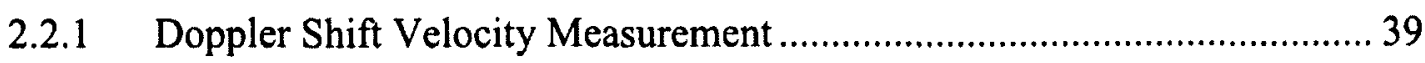


2.2.2 Cross Correlation Velocity Measurement.................................................. 40

2.3 Selection of Absorption Spectroscopic and Velocity Techniques ..................... 41

2.3.1 Selection of Absorption Spectroscopy Wavelength ................................. 42

Chapter 3 Background on MID-IR Absorption Spectroscopy and Technology ......... 47

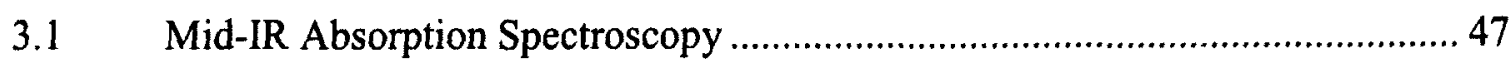

3.1.1 Measurement of Volume Fraction using DAS........................................... 49

3.1.2 Measurement of a Plume's Velocity through CCV with Mid-IR DAS ...... 51

3.1.3 Mid-IR Laser Source Survey ……………………................................... 53

3.1.4 Helium Neon Lasers .............................................................................. 54

3.1.5 Lead Salt Diode Lasers ........................................................................ 55

3.1.6 Quantum Cascade Lasers ....................................................................... 56

3.1.7 Difference Frequency Generation (DFG) Laser .......................................... 57

3.1.8 Optical Parametric Oscillators (OPO) ....................................................... 58

3.1.9 Motivation and Selection of the Mid IR Lasing Source ............................. 59

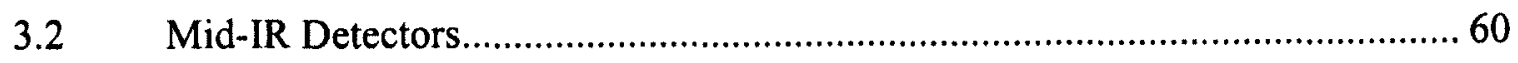

3.2.1 Motivation and Selection of the Mid IR detector ....................................... 63

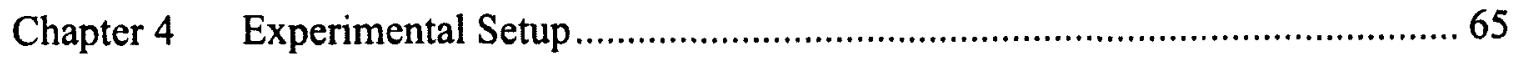

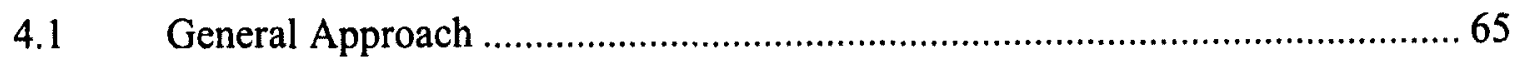

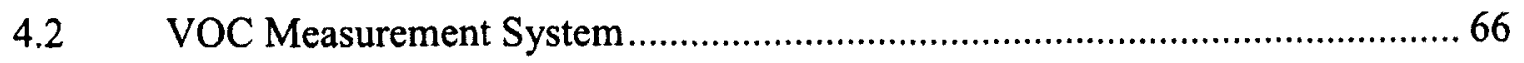

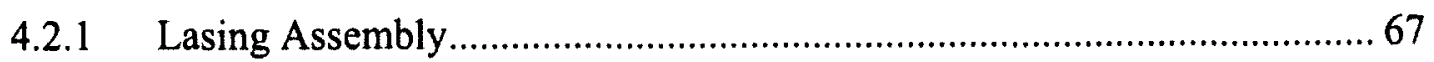

4.2.2 Test GasDelivery System....................................................................... 72

4.2.3 Detector Assembly .............................................................................. 75

4.3 Optical Power Conversion Electronics .............................................................. 76

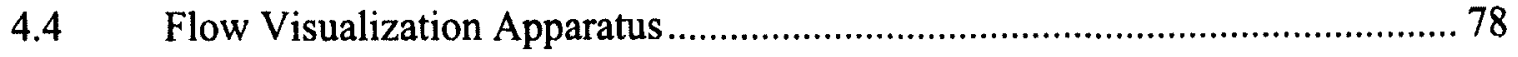




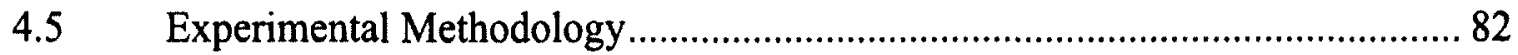

4.5.1 Line-Averaged Concentration Measurements …………………………...... 82

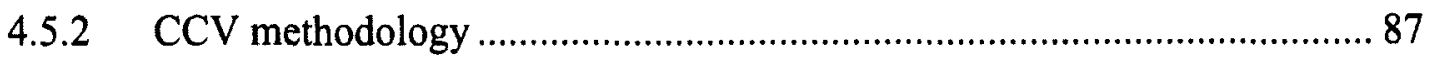

4.5.3 Mass Flow Transverse test................................................................ 91

4.5.4 Schlieren Imaging Methodology................................................................ 91

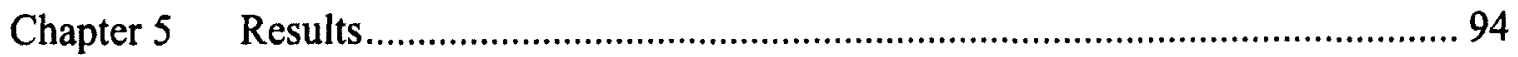

$5.1 \quad$ VOC Sensor Response .............................................................................. 94

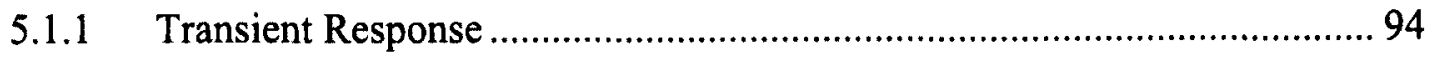

5.1.2 Random Noise Distribution ................................................................ 95

5.2 Concentration Measurement Sensitivity ........................................................... 98

5.3 CCV and Methane Flux Measurements ……….......................................... 102

5.3.1 Path integrated concentration measurements............................................. 103

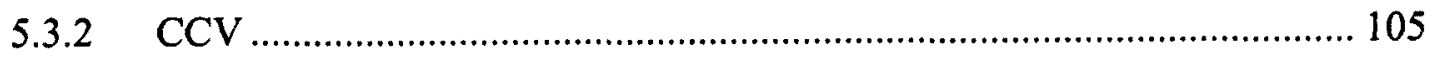

5.3.3 Line Flux Measurements....................................................................... 112

5.3.4 Mass flow measurements from traverse tests ......................................... 114

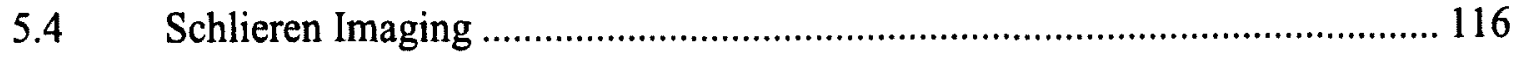

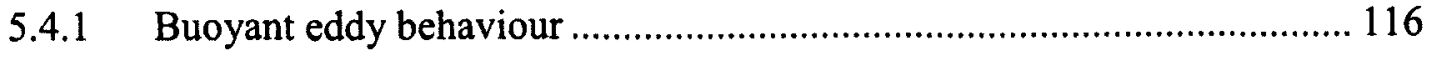

5.4.2 Plume path length ............................................................................ 119

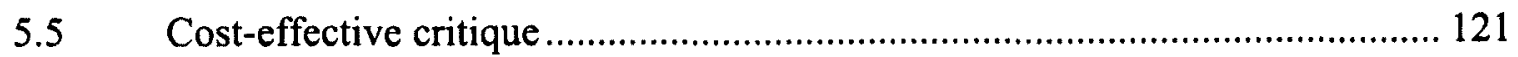

Chapter 6 Conclusions and Future Work ……………........................................... 123

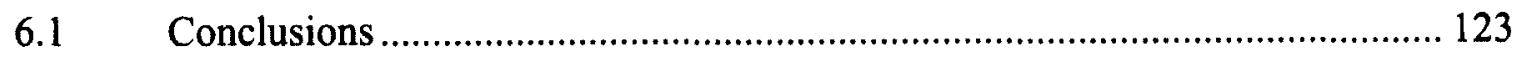

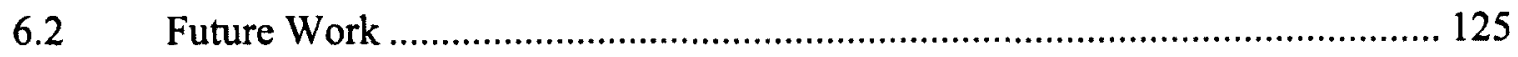

6.2.1 Detector-Amplifier Noise Performance ................................................... 125

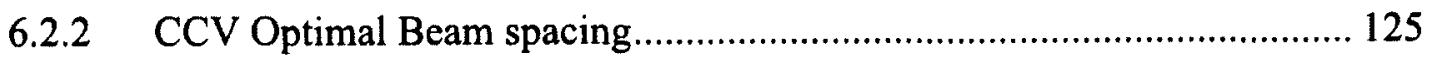




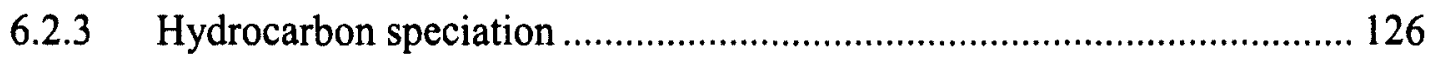

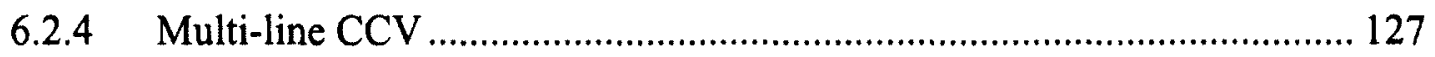

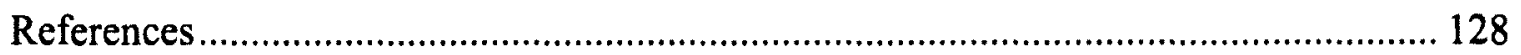

Appendix A Mechanisms of Evaporative Losses from Liquid Storage Tank .............. 133

A.1 Working Losses of Liquid Storage Tanks...................................................... 133

A.2 Standing Losses of Liquid Storage Tanks.................................................... 133

A.2.1 Standing Losses of Floating Roof Storage Tanks ................................... 134

A.2.2 Standing Losses of Fixed Roof and Horizontal Tanks ............................... 145

Appendix B Current Models for Estimating Emissions from Liquid Storage Tank....144

B.1 Emission Factors of Floating Roof Tanks....................................................144

B.1.1 Standing loses of Floating Roof Tanks ...............................................145

B.1.2 Working loses of Floating Roof Tanks ................................................151

B.1.3 Fugitive Emission Factors of "Landed" Floating Roof Tanks................152

B.2 Emission Factors of Fixed Roof Tanks and Horizontal Tanks ..........................157

B.2.1 Standing losses of Fixed Roof and Horizontal Tanks ............................157

B.2.2 Working losses of Fixed Roof and Horizontal Tanks............................159

Appendix C Amplifier PCB Design ……………................................................161

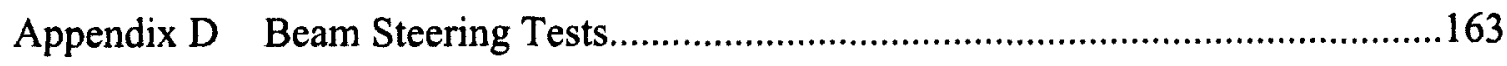




\section{List of Tables}

Table 1.1: Summary of Fugitive Emissions at Alberta Gas Plants as Measured with DIAL (Chambers, 2004). 31

Table 1.2: Measured vs. Estimated VOC Emissions a sweet gas plant (Chambers, 2004)

Table 1.3: Measured vs. Estimated VOC Emissions a sour gas plant (Chambers, 2004) 31

Table 2.1: Canister Sample from Condensate Tank Area at the Sour Gas Plant

(Chambers, 2004).

Table 2.2: The Optimal Spectral Regions (OSR) for the VOC emissions from an EFRT (AIST, 2010; Pouchert, 1981).

Table 3.1: Summary of Commercially Available Mid-IR Laser Source Survey (Pashotta, 2008; Tittel, Richter, \& Fried, 2003; Klingbeil, 2007; Cockburn, 2006; Kosterev, et al., 2008; Springholz, Schwarzl, \& Heiss, 2006; Weber, 1999)............................................. 60

Table 3.2: Theoretical SNR's for PV HgCgTe

Table 4.1: Relative uncertainties of the measured/calculated parameters ........................ 87

Table 5.1: Detector Assemblies transient response characteristics ................................... 94

Table 5.2: Time averaged concentration sensitivity ........................................................ 98

Table 5.3: Relative uncertainties of the measured/calculated parameters ..................... 101

Table 5.4: CCV Experimental Study Breakdown........................................................ 102

Table 5.5: The relative uncertainties of the mean path integrated concentration of

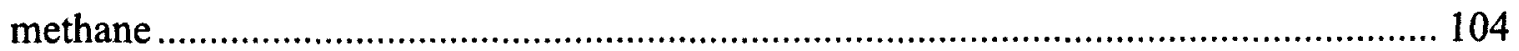

Table 5.6: The relative uncertainties of the measured line flux of $\mathrm{CH}_{4} \ldots \ldots \ldots \ldots \ldots \ldots \ldots \ldots . . . . .114$

Table 5.7: Methane mass flow measurements using a traverse …………....................... 116 
Table 5.8: The average plume path length measured from the post-processed Schlieren images (Units are in metres) ...................................................................................... 120

Table 5.9: Detailed Cost Breakdown of the Developed Sensor System.......................... 122

Table B.1: Rim seal loss factors (EPA, 1995) ……….............................................. 147

Table B.2: Clingage factors (EPA, 1995) ………………..................................... 152 


\section{List of Figures}

Figure 1.1: Typical Fixed Roof Tank (EPA, 1995) ...................................................... 24

Figure 1.2: External Floating Roof (EFRT) - Double-deck Type (Pasley\& Clark, 2000) 26

Figure 1.3: External Floating Roof Tank (EFRT) - Pontoon Type Roof (EPA, 1995) .... 27

Figure 1.4: Internal Floating Roof Tank (IFRT) (Land and Marine Project Engineering

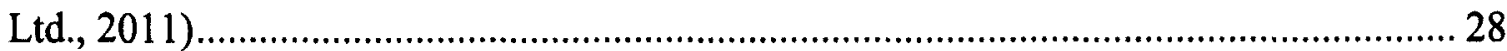

Figure 1.5: The honeycomb type roof construction (Long \& Garner, 2004) ................... 28

Figure 1.6: Typical underground horizontal storage tank (EPA, 1995) .......................... 29

Figure 1.7: Typical aboveground horizontal storage tank (EPA, 1995) ........................... 30

Figure 2.1: (left) FFT of the detector's signal with the $2 \mathrm{f}$ bandpass filter window; (right) the $2 \mathrm{f}$ component of the detectors signal that is proportional to target gas's concentration (Svensson et al., 2008) ............................................................................................... 37

Figure 2.2: DIAL Operational Methodology (Spectrasyne Ltd., 2010) ........................... 38

Figure 2.3: Illustration of the SOF method (Mellqvist et al., 2006) .................................. 39

Figure 2.4: General methodology of Velocity and Density measuring techniques (Mohamed \& Lefebvre, 2009)

Figure 2.5: Example of measuring the velocity of a turbulent jet with a CCV Probe. Two thermocouples placed d (cm) apart (Rockwell, Rangwala, \& Klein, 2009) ...................... 41

Figure 2.6: EFRT Headspace vapour composition and concentrations (Picard, 2009) .... 44

Figure 2.7: Transmission spectrum of Pentane (AIST, 2010) ………………………..... 45

Figure 2.8: Transmission spectrum of Hexane (AIST, 2010).......................................... 46

Figure 3.1: Variation of transmittance of $0.2 \%$ methane in air for different pressures at a fixed temperature of $300 \mathrm{~K}$ (Adapted from HITRAN 2008). 
Figure 3.2: Variation of transmittance of $0.2 \%$ methane in air for different temperatures at a fixed pressure of $101.3 \mathrm{kPa}$ (Adapted from HITRAN 2008).

Figure 3.3: A breakdown of individual maximum atmospheric absorption across the OSR for an $80 \mathrm{~cm}$ path length at STP. 51

Figure 3.4: Example of CCV on plume fluctuation; (a) Example of two transient signals; (b) The Cross correlation parameter as a function of time delays, seen maximized at 2 seconds. 52

Figure 3.5: Mid-IR Spectroscopic Sources and their operating ranges (Tittel et al., 2003)

Figure 3.6: Schematic of HeNe laser (Thorlabs, 2011) ..................................................5

Figure 3.7: Schematic of DFG laser source (Tittel, Richter, \& Fried, 2003) .................. 57

Figure 3.8: Optical Parametric Oscillation Lasing Methodology (Tittle et al., 2003)...... 59

Figure 3.9: The Operation Wavelength (A) and the Frequency Bandwidth (B) of commercially available Mid-IR detectors; the Blue region indicates (see A) the operational wavelength of interest for VOCs(Klingbeil, 2007)

Figure 3.10: Comparison of the $\mathrm{D}^{*}$ of various commercially available IR detectors (Piotrowski \& Rogalsk, 2007) 63

Figure 3.11: Schematic of simplified plume 64

Figure 4.1: Schematic of Proposed Experimental Apparatus to Measure a Line Flux through cross-correlation techniques; BS - Beam splitter, $\mathrm{M}$ - Turning mirror, CL Collimating lens.

Figure 4.2: Schematic of proposed experimental apparatus to determine concentration sensitivity; BS - Beam splitter, M - Turning mirror, CS - Collimating lens .66

Figure 4.3: Experimental apparatus (with plume generator) ............................................ 66

Figure 4.4: Lasing assembly of the VOC measurement sensor ......................................... 68

Figure 4.5: HeNe Laser - $30 \mathrm{~mm}$ cage alignment adapter ............................................. 68 
Figure 4.6: Spectral transmission curve of (Left) IR Neutral Density Filter ( $O D=0.3)$;

(Right) Specialized pellicle beam splitter

Figure 4.7: (Left) Spectral transmission of $\mathrm{f}=40 \mathrm{~mm} \mathrm{CaFl}_{2}$ Plano-convex lens; (Right) Spectral responsivity of the PVI-3.4 detector. 70

Figure 4.8: Spectral Reflectance curve for the gold plated mirror (Thorlabs Ltd., 2011) 71

Figure 4.9: (Left) Thermistor's electrical circuit; (Right) Calibrated temperature response

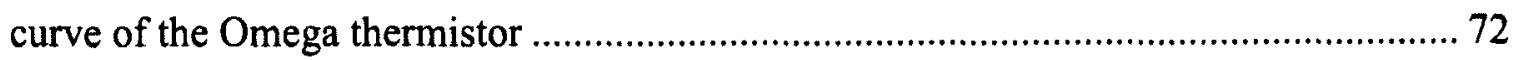

Figure 4.10: Schematic test gas delivery system and its components ............................ 72

Figure 4.11: Example of a calibration curve for Brooks MFC ......................................... 73

Figure 4.12: Plume generator for CCV spectroscopic study ........................................ 74

Figure 4.13: Spectroscopic flow-through cell................................................................ 74

Figure 4.14: Detector Assembly of the VOC measurement Sensor ................................. 75

Figure 4.15: Stage 1- Transimpedance amplifier with variable gain................................. 76

Figure 4.16: Stage 2 -ACextraction circuit with fixed (X2) gain.................................... 77

Figure 4.17: Stage 3 - AC amplification with variable gain........................................... 78

Figure 4.18: Custom "in-house built" detector amplifier .................................................. 78

Figure 4.19: A simple lens-based Schlieren setup (Atcheson, 2007) ............................... 79

Figure 4.20: Z-type Schlieren imaging system (Top-view) .............................................. 80

Figure 4.21: Parabolic mirrors used for Schlieren imaging system.................................. 81

Figure 4.22: (Left) Knife-edge assembly; (Right) Schlieren Image and acquisition camera .82

Figure 4.23: Mid-IR DAS methodology 82

Figure 4.24: Molar Absorption coefficient of $\mathrm{CH}_{4}$ distribution across the mid-IR OSR; the approximate location of the HeNe laser beams FWHM is highlighted in dashed red lines (HITRAN, 2008) 
Figure 4.25: The methodology of the experimental validation of CCV via direct

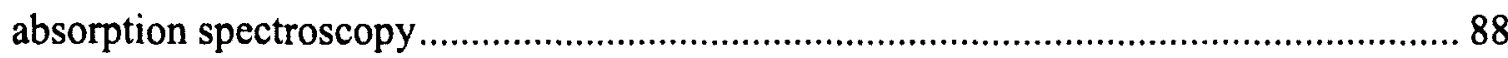

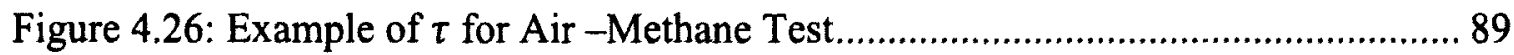

Figure 4.27: Example of calculated CL for both laser/detector lines (Left) and the $R_{x y}$

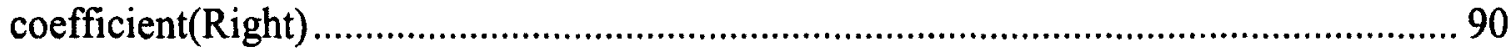

Figure 4.28: Schematic of the traverse test to measure the total mass flow of the plume 91 Figure 4.29: Example of the image processing used to gather spatial information of the plume. 92

Figure 4.30: Example of the combined binary image used to estimate length scales in the plume. 93

Figure 5.1: Detector response to a step change in incident laser radiation from 0 to $100 \%$ of the laser power. 95

Figure 5.2: Power spectrum distribution of the raw signals of(a) top detector and (b) bottom detector; The $\mathrm{AC}(60 \mathrm{~Hz})$ noise and its $n$ order harmonics are highlighted in dashed red boxes 96

Figure 5.3: (a) Power spectrum of the detector signal; (b) Power spectrum of the normalized detector signal. The AC $(60 \mathrm{~Hz})$ noise and its $n$ order harmonics are highlighted in dashed red boxes. 97

Figure 5.4: Measurements of the $\chi \mathrm{CH} 4$ for step inputs of 0 to (a) 0.05 and (b) $0.1 \ldots \ldots .99$ Figure 5.5: Measurements of the $\chi \mathrm{CH} 4$ of a Multi-Step concentration tests from 0.05 0.95 100

Figure 5.6: Measured vs. Set $\chi \mathrm{CH} 4$ along with the associated measurement uncertainty 101

Figure 5.7: Relative measured uncertainty as a function of set $\chi \mathrm{CH} 4$ 102

Figure 5.8: Measured path integrated concentration of methane for $\mathrm{Air}-\mathrm{CH}_{4}$ case study 104 
Figure 5.9: Measured path integrated concentration of methane for the Helium- $\mathrm{CH}_{4}$ case study 105

Figure 5.10: Standard deviation of the measured velocity vs. the CCV sample period for Air- $\mathrm{CH}_{4}$ mixtures at the (a) minimum and (b) maximum of experimental air flow range

Figure 5.11: Standard deviation of the measured velocity vs. the CCV sample period for Helium- $\mathrm{CH}_{4}$ flows at the (a) minimum and (b) maximum of experimental helium flow range 108

Figure 5.12: Example of a histogram of CCV extracted time delay for a test point. .... 109

Figure 5.13: (Left) the path integrated concentration of the (red) lower and (blue) upper detectors and the (Right) $R_{x y}$ time lag distribution for the corresponding 4000 samples for a Helium-Methane (1.5/0.1 SLPM) test run

Figure 5.14: Measured eddy velocities of the Air- $\mathrm{CH}_{4}$ plume as a function of methane mass flux

Figure 5.15: Measured eddy velocities for the Helium- $\mathrm{CH}_{4}$ case study as a function of the methane mass flux. 111

Figure 5.16: Measured eddy velocities of the Helium- $\mathrm{CH}_{4}$ case study as a function of the total mass flux 112

Figure 5.17: Measured Line Flux of Methane for the Air- $\mathrm{CH}_{4}$ mixtures. 113

Figure 5.18: Measured Line Flux of Methane for the Helium- $\mathrm{CH}_{4}$ mixtures. 113 Figure 5.19: Path integrated concentration measurements across the traverse path length 115

Figure 5.20: Plume eddy velocity measurements across the traverse path length 116 Figure 5.21: Time lapsed images ( $\Delta t=0.1 \mathrm{~s}$ ) of air (at $0.5 \mathrm{SLPM}$ ) and $\mathrm{CH}_{4}($ at $0.5 \mathrm{SLPM})$ 
Figure 5.22: Time lapsed images $(\Delta \mathrm{t}=0.1 \mathrm{~s})$ of air (at $1.5 \mathrm{SLPM})$ and $\mathrm{CH}_{4}$ (at $\left.0.5 \mathrm{SLPM}\right)$

Figure 5.23: Time lapsed images $(\Delta t=0.1 \mathrm{~s})$ of helium (at $1.5 \mathrm{SLPM}$ ) and $\mathrm{CH}_{4}$ (at 0.5 SLPM). 119

Figure 5.24 : Time lapsed images $\left(\Delta t=0.1 \mathrm{~s}\right.$ ) of helium (at 4.5 SLPM) and $\mathrm{CH}_{4}$ (at 0.5 SLPM).

Figure 5.25: Plume-Laser path length measurement of helium (at 4.5 SLPM) and $\mathrm{CH}_{4}$ (at 0.5 SLPM); (left) the intensity distribution across the laser beam spacing mid-point $(y=155, x=0$ - 424); (right) Average of a 100 post-processed Schlieren images 120

Figure A.1: Deck rim seals (EPA, 1995) ……………........................................... 135

Figure A.2: Typical Floating Roof Deck Fittings(EPA, 1995)...................................... 138

Figure A.3: Typical Floating Roof Deck Fittings (EPA, 1995)..................................... 140

Figure A.4: Slotted guide pole deck fitting (EPA, 1995) ............................................. 141

Figure A.5: Different configurations of a "landed" internal roof (EPA, 1995) .............. 142

Figure A.6: Section view of a typical underground horizontal storage tank (EPA, 1995) 143

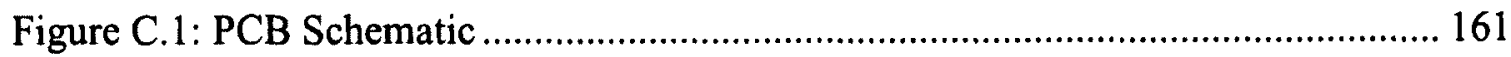

Figure C.2: PCB Layout ...................................................................................... 162 


\section{Nomenclature}

Symbol

$A_{D} \quad$ Active detector area $\left[\mathrm{cm}^{2}\right]$

$B_{i} \quad$ Systematic uncertainty of variable $X_{i}$

C Concentration $\left[\mathrm{mol} / \mathrm{m}^{3}\right]$

Path integrated concentration for the $\mathrm{n}$ detector

$C L_{n}(t)$

$\left[\mathrm{mol} / \mathrm{m}^{2}\right]$

$C_{i} \quad$ Concentration of the of the $\mathrm{i}^{\text {th }}$ gas $\left[\mathrm{mol} / \mathrm{m}^{3}\right]$

$D^{*}$

$D_{\text {beam }} \quad$ Laser Beam separation [m]

I Power of the transmitted radiation [W]

$I_{0} \quad$ Power of the incident radiation [W]

Detector signal that has normalized using the reference

$I_{N} / I_{\text {ref }}$

detector $[-]$

$I_{N} /\left.I_{\text {ref }}\right|_{0}$

The base detector signal that has normalized using the reference detector with no absorption [-]

$L \quad$ Path length through the target gas [m]

$m_{\mathrm{CH}_{4}}{ }^{\prime} \quad$ Line flux of methane $[\mathrm{g} / \mathrm{s}-\mathrm{m}]$

$M W_{\mathrm{CH}_{4}} \quad$ Molecular weight of methane $[\mathrm{g} / \mathrm{mol}]$

$N$

The total number of discrete samples i.e. the sample
First usage

Equation (3.6)

Equation (4.4)

Equation (3.1)

Equation (3.4)

Equation (3.2)

Equation (3.6)

Equation (4.19)

Equation (3.1)

Equation (3.1)

Equation (4.1)

Equation (4.1)

Equation (3.1)

Equation (4.21)

Equation (4.21)

Equation (3.5) 
period $[-]$

$\begin{array}{lll}P_{A} & \text { Barometric pressure }[\mathrm{Pa}] & \text { Equation (3.1) }\end{array}$

$P_{I} \quad$ Power of the incident radiation [W]

Equation (3.8)

$P_{i} \quad$ Precision uncertainty of variable $X_{i}$

Equation (4.4)

$Q_{L P M} \quad$ Volumetric flow rate [LPM]

Equation (4.12)

$Q_{S L P M} \quad$ Volumetric flow rate in STP conditions [SLPM]

Equation (4.12)

$R_{x y}$

Cross correlation parameter [-]

Equation (3.5)

$R \quad$ Universal gas constant [J/K-mol]

Equation (3.3)

$R$

Result

Equation (4.3)

$T \quad$ Temperature [K]

Equation (3.1)

$t_{v, 95 \%}$

$95 \%$ confidence t- value from the student's distribution

Equation (4.5)

$U_{X_{i}} \quad$ Uncertainty associated with variable $X_{i}$

Equation (4.3)

$V_{C C V} \quad$ Velocity from the CCV algorithm $[\mathrm{m} / \mathrm{s}]$

Equation (4.19)

$x$-axis

Defined as orthogonal to the laser light axis and the

plume flow direction

$y$-axis

Defined as parallel to the laser light axis and orthogonal to the plume flow direction

Defined as orthogonal to the laser light axis and parallel z-axis to the plume flow direction

$\Delta f \quad$ Frequency bandwidth of the detector $[\mathrm{Hz}]$

Equation (3.6)

$\Delta t_{C c V} \quad$ Time delay from CCV algorithm [s]

Equation (4.19) 


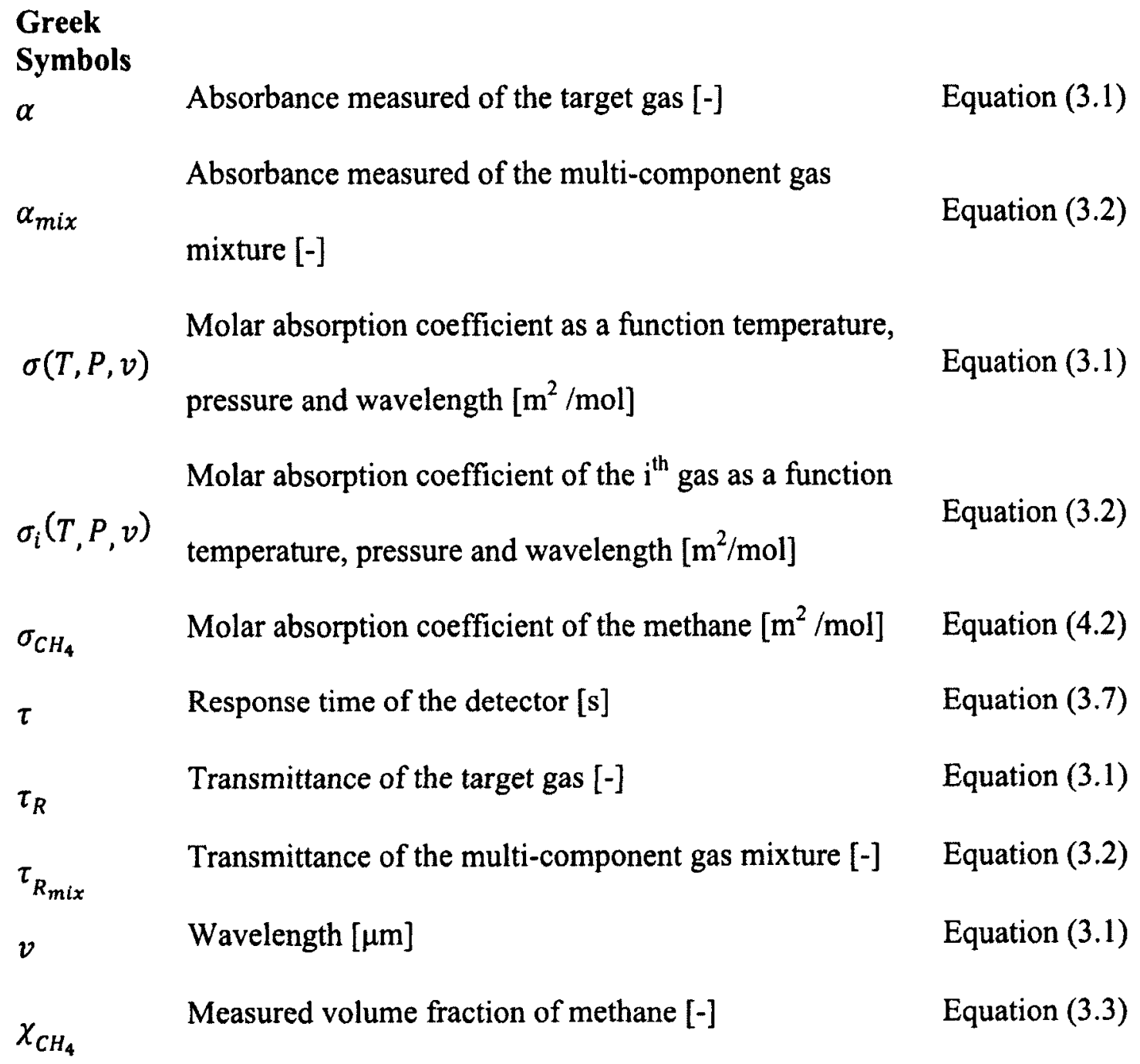

Molar absorption coefficient as a function temperature, $\sigma(T, P, v)$ pressure and wavelength $\left[\mathrm{m}^{2} / \mathrm{mol}\right]$

Equation (3.1)

Molar absorption coefficient of the $\mathrm{i}^{\text {th }}$ gas as a function

$\sigma_{i}(T, P, v) \quad$ temperature, pressure and wavelength $\left[\mathrm{m}^{2} / \mathrm{mol}\right]$

$\sigma_{\mathrm{CH}_{4}} \quad$ Molar absorption coefficient of the methane $\left[\mathrm{m}^{2} / \mathrm{mol}\right] \quad$ Equation (4.2)

$\tau \quad$ Response time of the detector [s] Equation (3.7)

$\tau_{R} \quad$ Transmittance of the target gas [-] Equation (3.1)

$\tau_{R_{m i x}} \quad$ Transmittance of the multi-component gas mixture [-] Equation (3.2)

$v \quad$ Wavelength $[\mu \mathrm{m}] \quad$ Equation (3.1)

$\chi_{\mathrm{CH}_{4}} \quad$ Measured volume fraction of methane [-] Equation (3.3)

Equation (3.2)

Acronyms

A/D Analog to digital Section (5.4.1)

$\begin{array}{lll}\text { AC } & \text { Alternating Current } & \text { Section (3.3.1) }\end{array}$

AIST Advanced Industrial Science and Technology $\quad$ Section (2.3.1)

API American Petroleum Institute Section (1.1)

$\begin{array}{lll}\text { AR Anti-reflective } & \text { Section (4.2.1) }\end{array}$ 


\begin{tabular}{|c|c|c|}
\hline BS & Beam splitter & Section (4.1) \\
\hline $\mathrm{CCV}$ & Cross correlation velocimetry & Section (2.2.2) \\
\hline $\mathrm{CL}$ & Collimating lens & Section (4.1) \\
\hline $\mathrm{CNC}$ & Computer numerical control & Section (4.2.1) \\
\hline DAS & Direct Absorption Spectroscopy & Section (2.1.1) \\
\hline DAQ & Data acquisition & Section (4.3) \\
\hline DC & Direct Current & Section (3.2.1) \\
\hline DFG & Difference Frequency Generation & Section (3.2.4) \\
\hline DIAL & Differential Absorption Lidar & Section (1.1) \\
\hline EFRT & External Floating Roof Tanks & Section (1.2) \\
\hline EPA & Environmental Protection Agency & Section (1.1) \\
\hline ET & étalon & Section (3.2.5) \\
\hline FTIR & Fourier Transform InfraRed (spectroscopy) & Section (2.3.1) \\
\hline GHG & Greenhouse Gas & Section (1.1) \\
\hline $\mathrm{HeNe}$ & Helium Neon & Section (3.2.1) \\
\hline HIPPO & HIAPER Pole-to-Pole Observations & Section (3.2.3) \\
\hline HITRAN & $\begin{array}{l}\text { High- resolution TRANsmission molecular absorption } \\
\text { database }\end{array}$ & Section $(2.1)$ \\
\hline ID & Inner diameter & Section (4.1) \\
\hline IFRT & Internal Floating Roof Tank & Section (1.2) \\
\hline IR & Infrared & Section (2.0) \\
\hline LST & Liquid Storage Tank & Section (1.1) \\
\hline
\end{tabular}




\begin{tabular}{|c|c|c|}
\hline NEP & Noise Equivalent Power & Section (3.3) \\
\hline NI & National Instruments & Section (4.3) \\
\hline NPT & National Physical Laboratory & Section (1.4) \\
\hline OD & Optical density & Section (4.2.1) \\
\hline OPO & Optical Parametric Oscillators & Section (3.2.5) \\
\hline OPAMPS & Operational amplifiers & Section (4.3) \\
\hline OSR & Optimal Spectral Region & Section (2.1) \\
\hline $\mathrm{PC}$ & Photoconductive & Section (3.3) \\
\hline PPLN & Periodically Poled Lithium Niobate & Section (3.2.4) \\
\hline PV & Photovoltaic & Section (3.3) \\
\hline QCL & Quantum Cascade Laser & Section $(3.2 .3)$ \\
\hline SLPM & Standard litres per minute & Section (4.2.2) \\
\hline SM & Semitransparent mirrors & Section (3.2.5) \\
\hline SNR & Signal to Noise Ratio & Section (2.1.2) \\
\hline TCEQ & Texas Commission on Environmental Quality & Section (1.4) \\
\hline TDL & Tunable Diode Laser & Section (2.1.1) \\
\hline TSM & Taylor series method & Section (4.5.1.1) \\
\hline UNC & Unified coarse & Section (4.2.1) \\
\hline VOC & Volatile Organic Carbon & Section (1.1) \\
\hline WMS & Wavelength Modulation Spectroscopy & Section (2.1.2) \\
\hline
\end{tabular}




\section{Chapter 1}

\section{Introduction}

\subsection{Motivation}

Liquid storage tanks (LST) are cylindrical metallic containers that operate at or very near atmospheric pressure (typical pressure difference of no more than a few inches of water). They are ubiquitous in the petrochemical industry where they are used to store a variety of organic products (hydrocarbon-based) throughout the production, refining, and distribution process. The American Petroleum Institute (API) and the United States Environmental Protection Agency (EPA) estimate that there are on the order of 700,000 petroleum storage tanks and about 1.3 million underground storage tanks in use in the United States alone (Myers, 1997).

Liquid storage tanks come in various designs and sizes, but are distinguished apart from other storage devices (such as pressure vessels) due to their interaction with the environment through operational fittings (e.g. open vents or pressure relief vents) and moveable seals. Environment Canada estimates that the emissions generated by liquid oil storage tanks contribute 2.2 percent of Greenhouse Gas (GHG) emissions and 31.5 percent of the Volatile Organic Carbon (VOC) emissions generated by the Canadian upstream oil and gas industry (Picard, 2009); amounting to $0.4 \%$ of national GHG emissions (Env. Can., 2008) and 9.97\% of national VOC emissions (Env. Can. 2010).

As detailed in Appendix A, the emissions from liquid storage tanks generally occur via three main mechanisms: working losses, standing losses and flashing losses. Working losses are the emissions that are generated by changes in the liquid level of the tank during the filling and emptying process, which push out or draw in gases. Standing losses (also known as breathing losses) are the emissions that are continually generated by varying ambient atmospheric conditions (i.e. fluctuations in ambient temperature, 
pressure, and insolent solar radiation causing expansion and contraction of the vapours within the tank, or changes in crosswind flow). These are further amplified through fitting leaks caused by operational wear. Flashing losses occur when organic liquid experiences a pressure drop causing previously dissolved gasses to be released, as most commonly occurs when pressurized transmission lines direct multi-component liquids into an atmospheric pressure storage tank.

Most current methods of estimating liquid storage tank emissions are based on a series of semi-empirical algorithms developed by the API and published by the EPA (1995). These models are widely used due to the lack of alternative models and the absence of economical sensor technology for measurement or monitoring. However, from a brief study conducted by Chambers et al. (2006) on five gas plants in Alberta using a Differential Absorption Lidar (DIAL), a laser-based optical method that can remotely measure the concentration of gases in the atmosphere, it was found that the measured daily emissions of methane and VOC's were four to eight times higher than the emission factor estimates. Unfortunately the DIAL technique is not an ecomically viable technology and cannot be utilized to measure emissions over longer time-scales (more than a day) without significant financial investment. Given the significance of VOC and GHG emissions from liquid storage tanks, there is an obvious need for quantitative models and measurement techniques that could be employed in reducing these important sources of fugitive emissions.

\subsection{Designs of Liquid Storage Tanks (LSTs)}

Liquid storage tank (LST) designs are generally classified based on their roof type and orientation. There are four main types of storage tanks used in the petrochemical industry. These are: fixed-roof tanks, Internal Floating Roof Tanks (IFRT), External Floating Roof Tanks (EFRT), and horizontal tanks.

\subsubsection{Fixed Roof Tank}

According to the EPA (1995), the most commonly used above ground LST is the fixed roof tank, which are the least expensive and the minimum acceptable device for the storage of hydrocarbon-based organic liquids. Figure 1.1 shows a typical fixed-roof tank 
design, which comprises a cylindrical steel shell with a cone or dome shaped roof that is permanently affixed to the tank walls, either by vapour tight weld seals or bolt/riveted seals (as seen with the older tanks)(EPA, 1995). Breather vents that allow gas exchange with the surrounding atmosphere are implemented to maintain pressure equalization during the filling and emptying process. Improvements such as pressure/vacuum valves have been implemented to prevent open flow circuits with the environment thereby mitigating some of the evaporative losses (Myers, 1997). However, Myers (1997) indicates that the large volume of air directly exposed to the organic liquid in the vapour space promotes high evaporative losses, especially for organic liquids with high saturation vapour pressures.

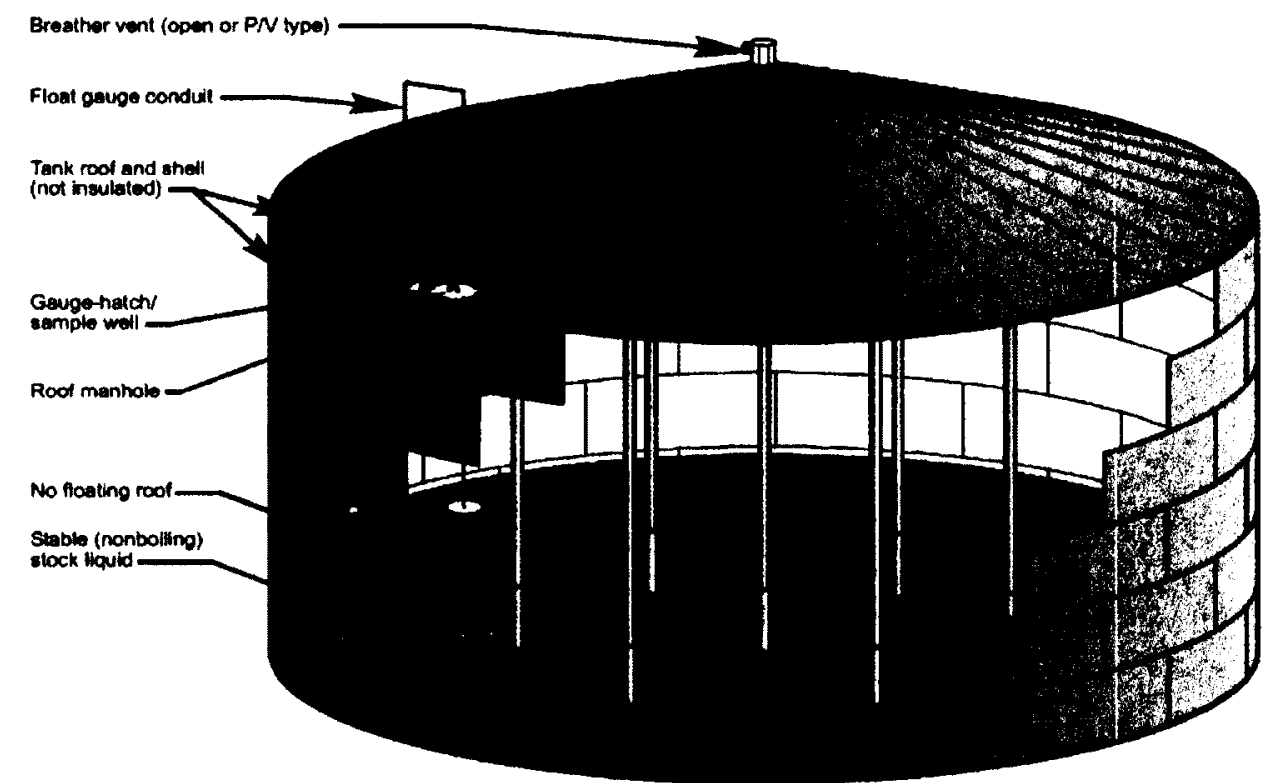

Figure 1.1: Typical Fixed Roof Tank (EPA, 1995)

\subsubsection{Floating Roof Tanks}

Floating roof tanks feature a moveable roof that floats on the surface of the liquid in the tank, thus reducing the vapour space above the liquid and minimizing gaseous exchange with the ambient environment. Since the 1920 s, these have been extensively used in the oil and gas industry (Myers, 1995). The implementation of floating roofs not only helped 
to reduce the fugitive emissions generated by storage tanks, but also helped to prevent vapour head space explosions that were quite common for fixed roof storage tanks (Myers, 1997).

There are two common implementations of the floating roof design: an open top configuration known as the External Floating Roof storage Tank (EFRT), and a fixed roof configuration known as the Internal Floating Roof storage Tank (IFRT). The designs of the EFRT and IFRT can be seen in Figure 1.2, Figure 1.3 and Figure 1.4. Although floating roofs have helped reduce the emissions generated by storage tanks, the emissions are still significant (EPA, 1995).

\subsubsection{External Floating Roof Tank (EFRT)}

An External Floating Roof Tank (EFRT) consists of an open-topped cylindrical steel shell that surrounds a floating shell (roof). The floating roof is generally manufactured from steel and rests on the liquid surface, rising and falling along with liquid level in the tank. There are two widely implemented floating roof designs in these tanks: the pontoon floating roof and the double-deck floating roof. Myers (1997) indicates that the pontoon deck design, shown in Figure 1.3 is the most commonly used roof design used in tanks up to about 65 metres in diameter. As described by Long \& Garner (2004), the roof can be broken down into two main parts, the deck and outer annular pontoon. The deck is generally manufactured from $3 / 16$ inch steel plates that are seal welded on the weather exposed side and stitch welded on the fluid exposed side. Depending on the fluid being stored, crevice corrosion can be expected to occur on the lower surface, which can be mitigated with seam welding. The outer annular pontoon ensures proper buoyancy and provides the majority of the structure's rigidity. However, due to the inherent flexibility of plate steel, the pontoon design is prone to the creation of vapour space at the center of the roof and experiences fatigue cracking from wind excited deflections.

The double-deck design shown in Figure 1.3 is the heaviest and the most durable of the external roof designs (due to its relatively high rigidity), and is the most economical choice for smaller tanks ( $\sim 10$ metres) and very large tanks (60 to over 90 metres) (Myers, 1997). The roof is constructed from two seam welded decks that are separated by a series of circumferential and radial bulkheads. The bulkheads are seal 
welded to create vapour tight compartments. The double-deck roof's rigid design prevents any vapour space creation due to deck deformation and limits the possibility of wind excited fatigue cracking (Long \& Garner, 2004). Additionally, the separation space between decks also acts like an insulation layer, inhibiting solar heat transfer to the stored organic fluid, which prevents large temperature variations in the storage fluid leading to a reduction in emissions (Myers, 1997; Long \& Garner, 2004).

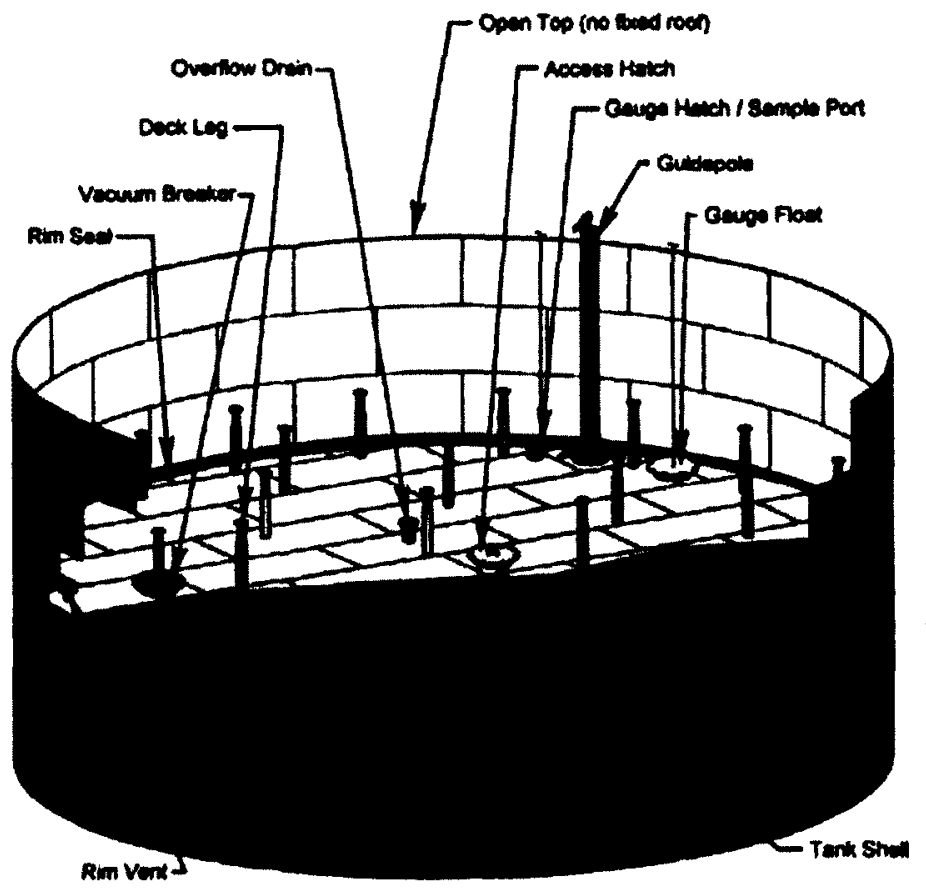

Figure 1.2: External Floating Roof (EFRT) - Double-deck Type (Pasley \& Clark, 2000) 


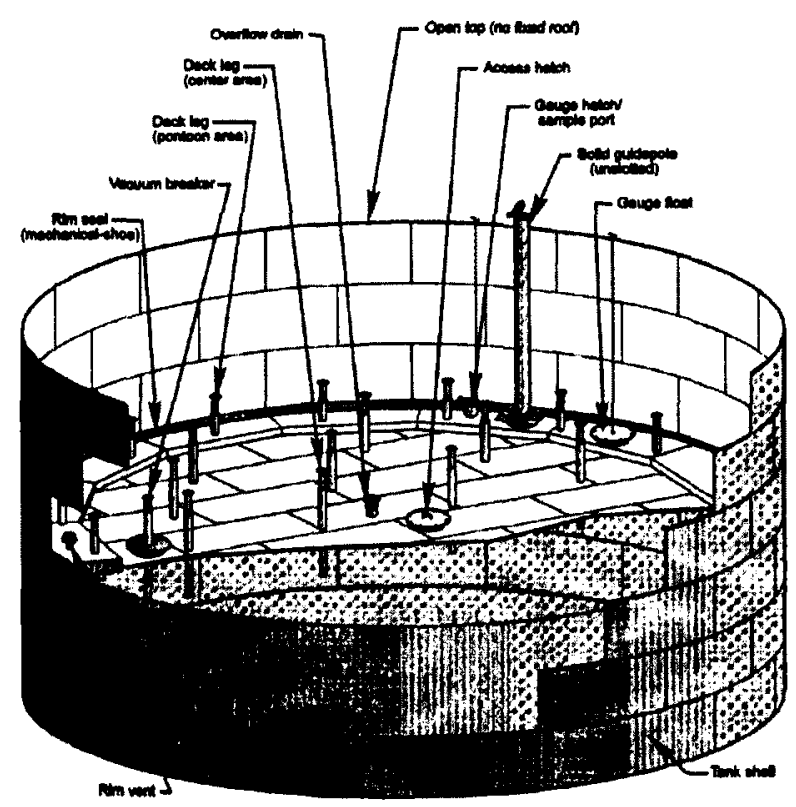

Figure 1.3: External Floating Roof Tank (EFRT) - Pontoon Type Roof (EPA, 1995)

\subsubsection{Internal Floating Roof Tank (IFRT)}

The typical Internal Floating Roof Tanks (IFRT) design is a combination of an internal buoyant deck and a fixed outer roof structure. The buoyant deck is generally made of either steel or aluminum, and rests directly on the liquid surface limiting the available vapour space. The enclosed nature of the roof allows for significant relaxation of the roof's design requirements under the API standards (Myers, 1997; Long \& Garner, 2004). This is mainly due to the minimal loading effect on the floating roof from the surroundings (i.e. wind, precipitation etc.). Myers (1997) stated that most of steel IFRTs were converted from EFRTs, by simply constructing a fixed roof above the tank.

Aluminum internal floating roofs are generally manufactured in two fashions: skin/pontoon and honeycomb (Myers, 1997). The skin and pontoon roof is constructed from closed end aluminum pipes that are mounted in a fixed frame and covered with a skin of aluminum sheets. Due to the pipe pontoon design, there is approximately a 6 inch gap between the deck and liquid surface, which leads to the creation of a vapour space that promotes emissions from degraded deck fittings (Myers, 1997).

The internal honeycomb panel roof is constructed from extruded aluminum sections that are sealed off on either end by aluminum sheet metal. The deck design 
employs a frame constructed from extruded aluminum to house the individual honeycomb panels, as shown in Figure 1.5. This allows the deck to lie on the organic liquid surface preventing the formation of vapour space under the deck (Myers, 1997).

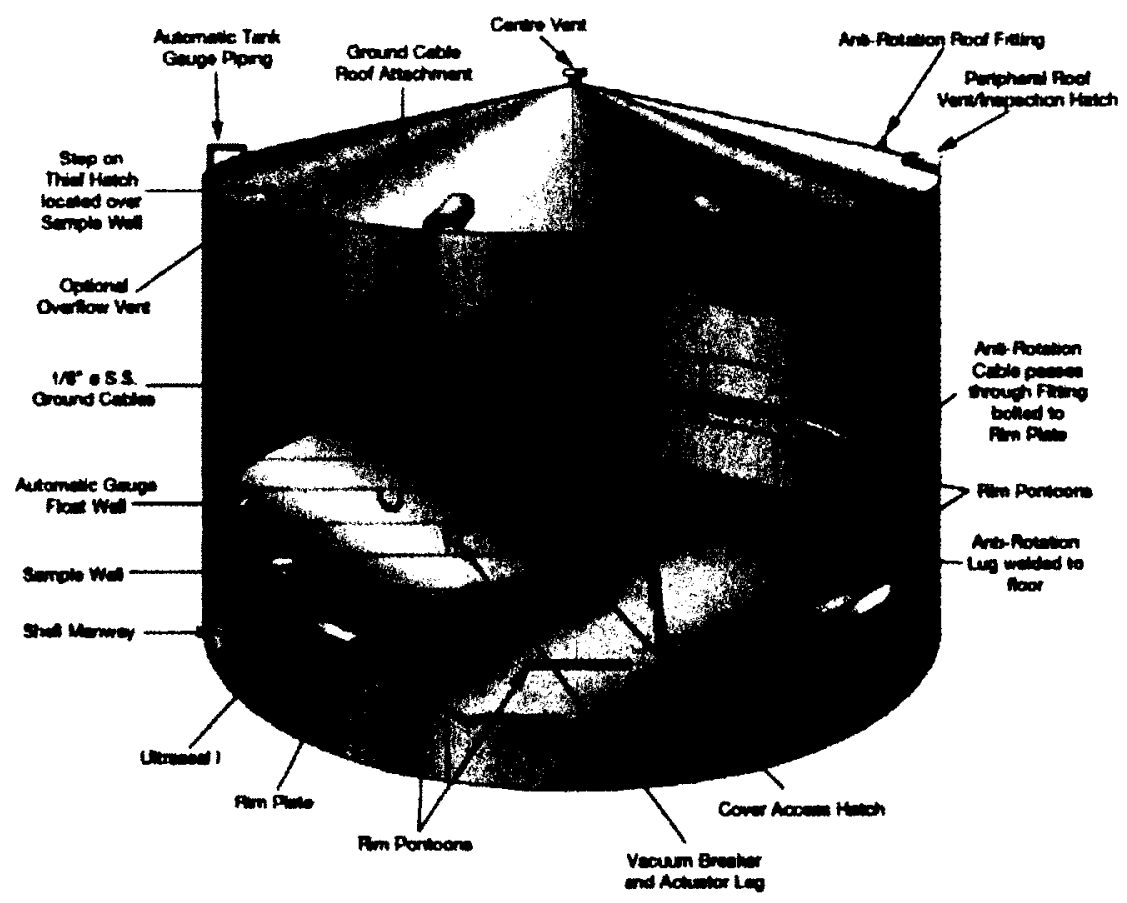

Figure 1.4: Internal Floating Roof Tank (IFRT) (Land and Marine Project Engineering Ltd., 2011)

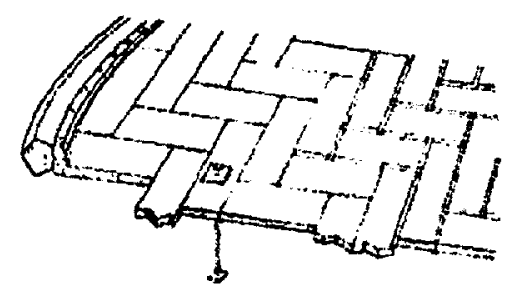

Panel arrangement

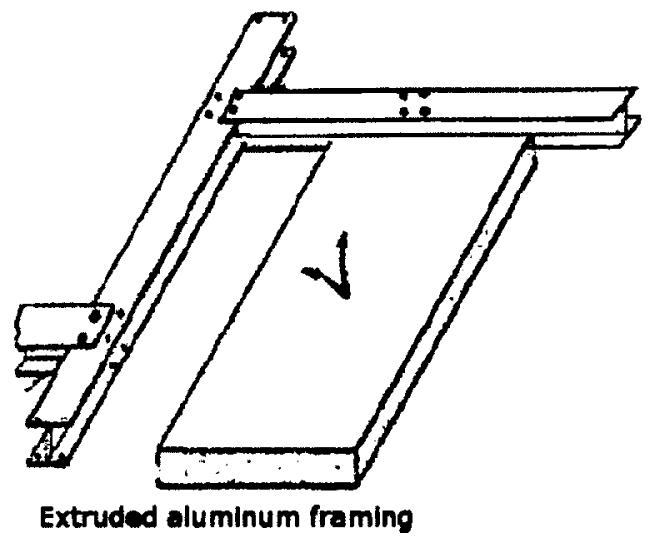

Figure 1.5: The honeycomb type roof construction (Long \& Garner, 2004) 


\subsubsection{Horizontal Storage Tanks}

Horizontal tanks, shown in Figure 1.6 and Figure 1.7, are usually manufactured from steel and are used in both above ground and underground service. Theses tanks are used for smaller-volume storage, such as for commercial distribution at gasoline stations. The horizontal tank's interaction with its surroundings is quite similar to fixed roof tanks, where pressure/vacuum vents allow bi-directional flow between the vapour space and the atmosphere to equalize the pressure during filling-emptying cycles, diurnal temperatures and barometric changes. In addition to pressure vents, horizontal tanks are typically also fitted with gauge hatches, access holes, and sample wells and allow for day-to-day operation and maintenance (EPA, 1995).

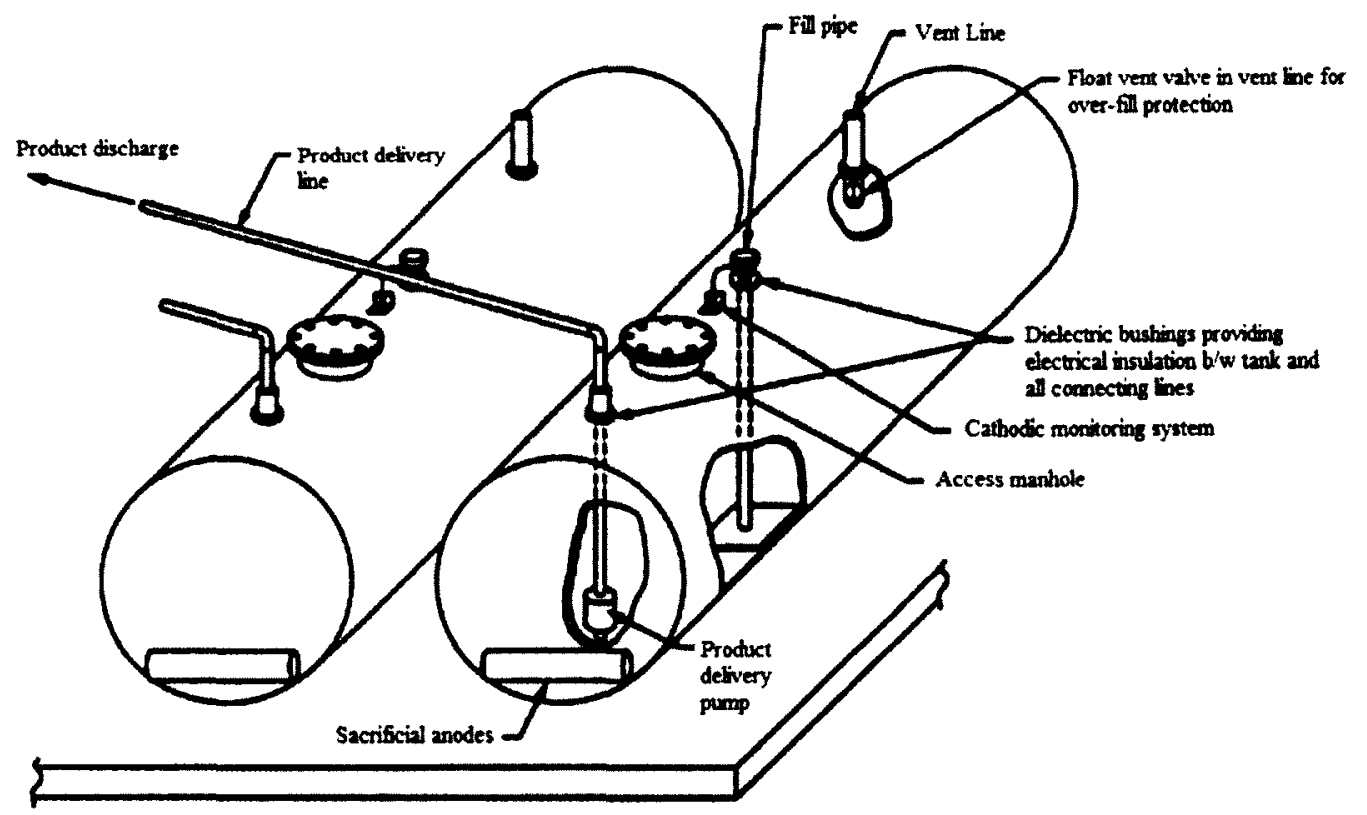

Figure 1.6: Typical underground horizontal storage tank (EPA, 1995) 


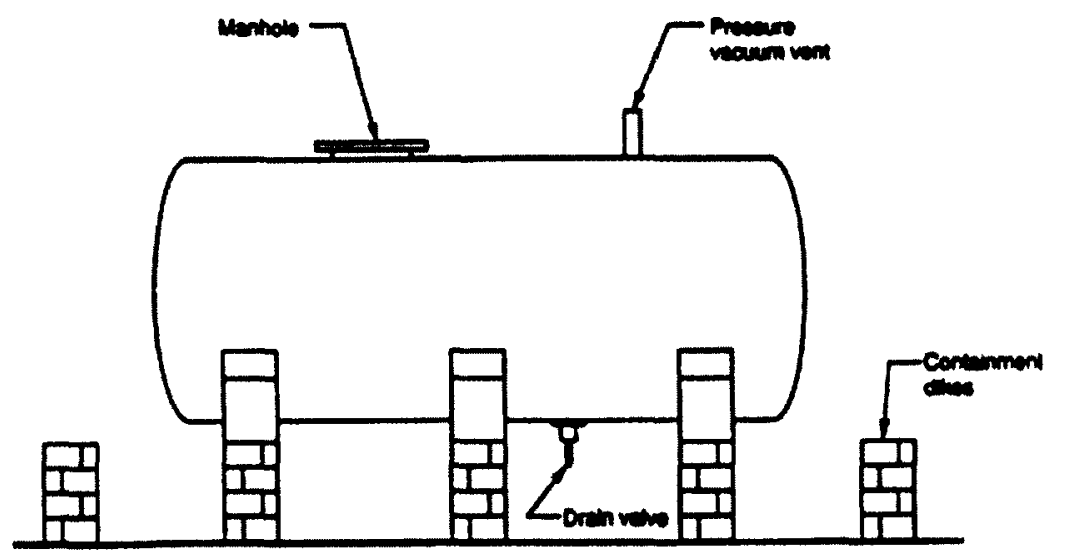

Figure 1.7: Typical aboveground horizontal storage tank (EPA, 1995)

\subsection{Previous Comparisons of Estimated Emissions from Liquid Storage Tanks with Field Measurements using Differential Absorption LIDAR (DIAL)}

In 2003 and 2004, Spectrasyne Ltd. conducted a survey of fugitive emissions of five gas processing plants in Alberta using Differential Absorption LIDAR (DIAL) (Chambers A. K., 2004). The DIAL technique allows concentration data for a target species to be measured along an open-path laser line as describe more completely in Section 2.1.3. By scanning along multiple lines and combining spatially resolved concentration data with wind measurements, emissions flux through a plane (typically at a facility boundary) can be estimated. Using DIAL at five sites in Alberta, (compared to the current estimates) significant levels of fugitive emissions were observed as summarized in Table 1.1.

It should be noted that the DIAL measurements were found to have reasonable accuracy following two validation studies conducted in Alberta (Chambers A. K., 2004), and one performed by the National Physical Laboratory (NPT)(Robinson et al., 2011). The two studies conducted in Alberta, one of sulphur dioxide from a tail gas incinerator at a gas processing facility and the other of nitric oxide plume from a gas turbine plant demonstrated that the emission mass flux measured by DIAL were found to lie between $-11 \%$ and $1 \%$ of the mass flux rate determined by in-stack monitoring. The NPL validation tests were conducted similarly, where the DIAL measured flux was compared 
with controlled stack releases. They measurements were found to agree within $\pm 15 \%$ and $\pm 22 \%$ of the controlled release rates of methane and benzene respectively.

Table 1.1: Summary of Fugitive Emissions at Alberta Gas Plants as Measured with DIAL (Chambers, 2004)

\begin{tabular}{|c|c|c|c|c|c|}
\hline $\begin{array}{c}\text { Gas } \\
\text { Plant }\end{array}$ & $\begin{array}{c}\text { Year } \\
\text { Surveyed }\end{array}$ & $\begin{array}{c}\text { Plant Nominal } \\
\text { Flow Rate } \\
\left(\mathbf{1 0}^{\mathbf{6}} \mathbf{~ m}^{\mathbf{3}} / \mathbf{d}\right)\end{array}$ & $\begin{array}{c}\mathbf{C H}_{\mathbf{4}} \\
\text { Emissions } \\
(\mathbf{k g} / \mathbf{h r})\end{array}$ & $\begin{array}{c}\mathbf{C}_{\mathbf{2}} \\
\text { Emissions } \\
\mathbf{( k g / h r})\end{array}$ & $\begin{array}{c}\text { Benzene } \\
\text { Emissions } \\
\mathbf{( k g / h r})\end{array}$ \\
\hline A & 2003 & 1.45 & $\begin{array}{c}8 \\
(\text { tanks only) }\end{array}$ & 38 & - \\
\hline B & 2003 & 3.5 & 104 & 42 & - \\
\hline C & 2003 & 10 & 146 & 342 & - \\
\hline C & 2004 & 10 & 100 & 58.4 & 0.24 \\
\hline D & 2003 & 6 & 124 & 86 & - \\
\hline E & 2004 & & 144 & 41 & 0.06 \\
\hline
\end{tabular}

Table 1.2: Measured vs. Estimated VOC Emissions a sweet gas plant (Chambers, 2004)

\begin{tabular}{|c|c|c|}
\hline Item & $\begin{array}{c}\text { Estimated Emissions } \\
\text { (tonnes/yr) }\end{array}$ & $\begin{array}{c}\text { DIAL Measured } \\
\text { (tonnes/yr) }\end{array}$ \\
\hline Tanks & 4.91 & 11.0 \\
\hline Flare & 0.72 & 8.5 \\
\hline $\begin{array}{c}\text { Combustion } \\
\text { sources }\end{array}$ & 4.93 & -- \\
\hline Plant fugitives & 2.69 & 107.1 \\
\hline Other & 1.63 & 2.6 \\
\hline Total & 14.28 & 129.2 \\
\hline
\end{tabular}

Table 1.3: Measured vs. Estimated VOC Emissions a sour gas plant (Chambers, 2004)

\begin{tabular}{|c|c|c|}
\hline Item & $\begin{array}{c}\text { Estimated Emissions } \\
\text { (tonnes/yr) }\end{array}$ & $\begin{array}{c}\text { DIAL Measured } \\
\text { (tonnes/yr) }\end{array}$ \\
\hline Tanks & 0.69 & 309 \\
\hline Flare & 1.26 & 42 \\
\hline $\begin{array}{c}\text { Combustion } \\
\text { sources }\end{array}$ & 8.38 & \\
\hline Plant fugitives & 84.03 & 193 \\
\hline Total & 94.36 & 545 \\
\hline
\end{tabular}


Measured fugitive emission rates obtained using DIAL were generally much higher than predicted values using standard emission factor approaches, detailed in Appendix B (Chambers, 2004). Table 1.2 and Table 1.3 show a direct comparison of measured and estimated emission rates for specific plant components. For liquid storage tanks specifically, DIAL measured emission rates were 2.2 and 450 times greater than estimated emission rates (11.0 vs. 4.91 and 309 vs. 0.69 tonnes/yr respectively). More generally, evaporative losses from liquid storage tanks contributed to about $36 \%$ methane and $57 \%$ VOC of the total site emissions, notably one of the largest contributors to the total plant emissions (Chambers, 2004). This trend has also been observed overseas by experimental findings published by the IMPEL Network, where DIAL studies indicated that $\sim 42 \%$ of the total VOCs released at an oil refinery were attributable to liquid storage tanks (IMPEL Network, 2000).

While the DIAL studies did not present losses specific to the evaporative losses from landed roofs, recent data published by the Texas Commission on Environmental Quality (TCEQ) states the landing losses from tanks have been seriously under-reported, amounting up to 7250 tons per year in the Houston-Galveston area alone (Schanbacher, 2007).

From the comparisons of the DIAL measurements to the current EPA estimates, the need for further improvement in the estimation models can be seen; however, the floating roof storage problem, due a significant number of factors (wind speed, weather degradation, etc.) is an immensely complex modeling problem. In addition, while DIAL measurements indicate that the emissions are significantly higher than predicted, the tests were necessarily conducted over a short periods of time (relative to a full operating year), and thereby cannot fully represent the evaporative loss distribution over the annual operating period; due to the fact that there is no statistical basis for correlating annual emissions from a single instance measurements. This argument, presented by Ferry (Bosch Jr. \& Logan, 2006), while holding merit in a statistical sense does not answer the doubt of the EPA estimates presented by several snap-shot studies of fugitive emissions of storage tanks. It is from these opposing arguments, that the need for an economical 
measurement system that can quantify the instantaneous emissions from liquid storage tanks over the annual working period is required.

\subsection{Experimental Objective}

The goal of this thesis was to design and develop a cost-effective measurement system that uses Mid-IR absorption to measure the velocity of a transient hydrocarbon plume in a lab-setting through cross correlative techniques. The proposed research is a stepping stone to creating a real-time measurement system that uses a multi-line Mid-IR absorption technology to measure in-field fugitive emissions mass flow rates from storage tanks in a quantitative manner. This research will ultimately provide the experimental findings that could be used to update the API correlations currently used by industry. Having better models and diagnostics could reduce emissions by enabling faster identification and repair of leaks and seals, improved operating procedures to minimize activities associated with unintended emissions and better data from which to justify economics of more aggressive mitigation strategies (e.g. large scale vapour recovery systems).

\subsection{Organization of Thesis}

The preceding discussion in Chapter 1 contains background information on liquid storage tanks and their corresponding hydrocarbon evaporative losses. The official estimates for liquid storage tanks are presented along with experimental findings from DIAL that demonstrate their shortcomings and the need for monitoring technologies to improve the accuracy of the estimates and allow for shorter leak detection timescales. In Chapter 2, the background of various remote sensing techniques that implement absorption spectroscopy to quantify background hydrocarbon concentrations in the atmosphere is presented in the context of an economical in-field implementation. The latter half of the chapter discusses motivation and selection of the technique to measure both the concentration and velocity for the proposed in-lab VOC plume measurement sensor. In Chapter 3, the background and theory of the Mid-IR spectroscopic technique selected for the VOC measurement sensor is presented. The theory of commercially available Mid-IR spectroscopic components is presented along with the motivation and selection for the in- 
lab measurement sensor in the latter part of the chapter. Chapter 4 presents detailed makeup of the two experimental setups used to benchmark the sensor and the selected technique for a future full-scale VOC mass flux measurement sensor. Chapter 5 presents the results and the overall performance of the in-lab setup and the sensor's concentration sensitivity and associated uncertainty. The performance of the cross-correlative technique is discussed in the latter part of Chapter 5. Finally, the conclusions of this research project and future work is presented in Chapter 6. 


\section{Chapter 2}

\section{Remote Sensing of Evaporative Losses from}

\section{Liquid Storage Tanks}

To the author's knowledge, there are no specific direct measurement techniques available for liquid storage tanks. However, there is a range of different diagnostics that are relevant. These are briefly reviewed here. In addition, qualitative techniques such as IR camera surveying or downwind sampling can be utilized to detect or monitor emissions without quantifying magnitudes (Chambers, 2004). While infrared cameras offer a fast technique to detect leaks, they are labour intensive and are adversely affected by complex backgrounds, lighting conditions and their own inherent detection limits. Because these approaches are non-quantitative they are not considered further.

\subsection{Absorption Spectroscopy}

Absorption spectroscopy uses measured absorption of electromagnetic radiation passing through a sample to calculate mean concentration of a target species along the optical path. Every molecule exhibits its own absorption spectrum which is based on the quantum mechanical change induced by the absorbed electromagnetic radiation. Spectral absorption strength data as a function of optical frequency, temperature, and pressure are available for many different molecules in databases such as HITRAN (2008). The absorption of infrared (IR) electromagnetic energy by molecules is restricted to the energy changes between the rotational and vibrational (ro-vibration) quantum states. If the frequency of the incoming electromagnetic radiation matches the ro-vibrational frequency of the target molecule, the electromagnetic energy will be absorbed (So et al. 2009). By measuring the intensity of the radiation before and after passing through the 
test sample, the absorption spectrum can be experimentally ascertained. The spectral region with the highest absorbance values defines the Optimal Spectral Region (OSR), which for a given molecule can be targeted to measure the concentration of the molecule with high accuracy (Hollas, 2004). The following sections briefly describe various types of absorption spectroscopy that have the capacity to be implemented in the field

\subsubsection{Direct Absorption Spectroscopy (DAS)}

Direct Absorption Spectroscopy (DAS) is one of the simpler implementations of absorption spectroscopy. There are two main types of DAS: monochromatic DAS and scanned-wavelength DAS. Monochromatic DAS uses a fixed-wavelength source to probe a single spectral absorption peak. Scanned-wavelength DAS is generally accomplished using a Tunable Diode Laser (TDL), which allows for rapid scanning of the optical wavelength across an absorption feature to obtain both baseline and peak intensity measurements that are proportional to the concentration of the target gas. The wavelength scan is achieved by varying the injection current to the laser diode. Scannedwavelength DAS permits discrimination of the target gas from background absorption from other species (such a dust particulates). However, DAS is highly susceptible to noise introduced through the laser sources and/or the optical system. In general, reference detectors are utilized to measure the laser's power before absorption by the target gas to establish a proper base datum (which can drift over time). The use of a reference detector also enables removal of excess laser noise from the measured signals (Rieker, 2009).

\subsubsection{Wavelength-Modulation Spectroscopy (WMS)}

Wavelength Modulation Spectroscopy (WMS) is similar to scanned-wavelength DAS where the absorption feature of the target gas is scanned across a (wavelength) bandwidth; however, WMS superimposes a secondary high modulation frequency, low amplitude sinusoid signal in addition to the (relatively) slower ramp signal. The interaction between a rapidly alternating wavelength and a nonlinear absorption feature creates a series of $\mathrm{n}^{\text {th }}$ order harmonics of the absorption feature at periods of the modulation frequency (Svensson et al., 2008). By measuring the $2^{\text {nd }}$ order harmonic, it is 
possible to obtain a Signal to Noise Ratio (SNR) 2 - 100 times greater than DAS, because the signal of interest lies far beyond the region that is degraded by $1 / \mathrm{f}$ noise generated by the detectors, seen in the Figure 2.1 (Svensson et al., 2008).
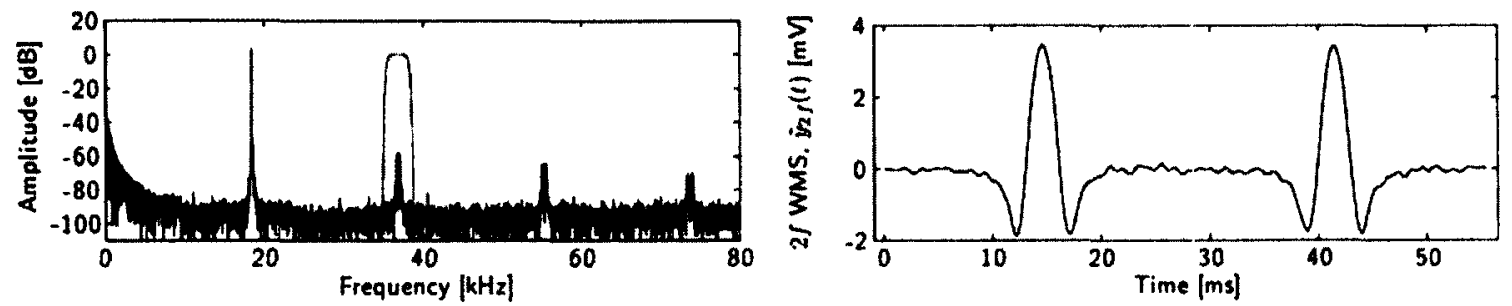

Figure 2.1: (Left) FFT of the detector's signal with the $2 \mathrm{f}$ bandpass filter window; (Right) the $2 \mathrm{f}$ component of the detectors signal that is proportional to target gas's concentration (Svensson et al., 2008).

\subsubsection{Differential Absorption Light Detection and Ranging (DIAL)}

Differential Absorption Light Detection and Ranging (DIAL) is an optical measurement technique derived from absorption spectroscopy that uses laser pulses at two different wavelengths to remotely measure the concentration of gases present in the atmosphere up to a range of $2 \mathrm{~km}$ with part per billion accuracy (Chambers, 2004). The system uses a series of collection mirrors and lenses to direct the laser pulses at a specific target area and to collect the back scattered light from particles or aerosols in the atmosphere. One wavelength is selected where the target species has high absorbance and one wavelength is chosen where the target species has little or no absorbance. This allows a baseline (no absorbance) intensity measurement to be incorporated directly into calculations so that measured concentrations are not influenced by absorption by particulate (i.e. dust) along the optical path. By measuring the intensity and the time delay of the collected light (time interval between the emitted pulse and received pulse), the concentration profile over the beam path can be measured. By employing a scanning telescope/mirror system, the laser pathway can be angularly tuned (within the vertical plane) which allows for generation of high accuracy concentration maps (Spectrasyne Ltd., 2010)

From the concentration maps, the mass flux can be estimated by multiplying by wind speed (measured separately). By tuning the laser wavelength, the DIAL system can be adjusted to measure specific species, such as methane or benzene, at accuracies of \pm 15 
and \pm 22 percent respectively or an entire group of species (Bosch Jr., J., \& Logan, T., 2006). When measurements are conducted on a mixture of gases, such as hydrocarbons, sorption tubes are placed within the targeted plume to sample the gases, which are separately analyzed to determine the detailed species compositions and their average molecular weight which allows for mass flux measurements of multi-component species (Spectrasyne Ltd., 2010).

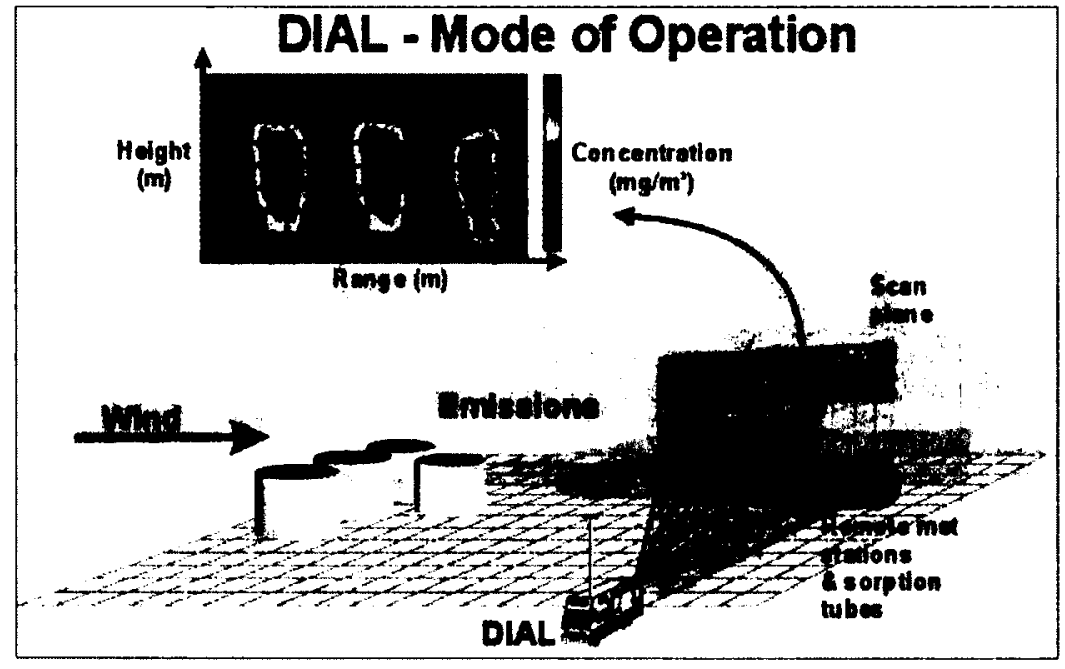

Figure 2.2: DIAL Operational Methodology (Spectrasyne Ltd., 2010)

Spectrasyne Ltd. has commercially operated a mobile DIAL system known as the Environmental Surveying System (ESS) across Europe for the past 15 years and shipped to other countries for surveying contracts. The technological investment required for the construction of ESS systems is estimated to cost \$3-4 million. The monetary investment required for this technology limits its wider use.

\subsubsection{Solar Occultation Flux (SOF) Method}

Solar Occultation Flux is an optical measurement technique that utilizes the sun as the light source for quantitative measurements. The broadband infrared spectrum of the sun is recorded by a commercially built spectrometer mounted on the roof of a vehicle. To obtain emission flux data, the vehicle is driven so that the detected solar light moves across the actual emission plume (a function of sun position and wind direction). The 
emission flux is determined as the integrated sum of the concentration measurements (from the spectrometer) multiplied by the wind speed. This technique was developed to satisfy the need for a cost effective emission measuring technology due to the fact that the DIAL technique is generally cost-prohibitive. The SOF method was demonstrated to provide quick, cost effective measurements of alkanes as well as ammonia, carbon monoxide, formaldehyde, hydrogen chloride, by a study conducted (2002-2004) on three oil refineries in Sweden (Mellqvist et al., 2006). In this same study, crude-oil storage tanks and liquid product tanks were found to contribute to $31 \%$ and $32 \%$ respectively of the total site emissions. However, the SOF method has been documented to have measurement errors up to $25 \%$ in some cases, caused mainly by uncertainties in the wind field.

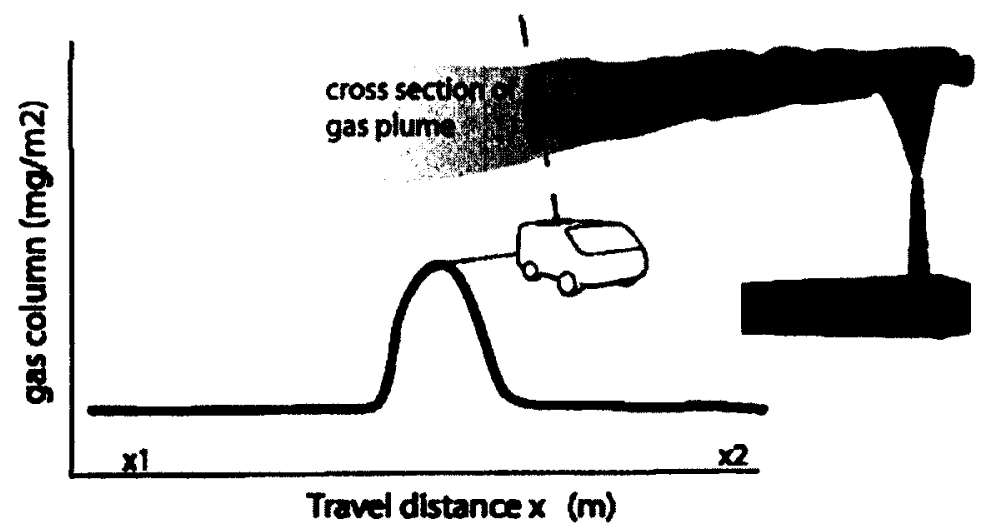

Figure 2.3: Illustration of the SOF method (Mellqvist et al., 2006)

\subsection{Velocity Measurement Techniques using Absorption Spectroscopy}

\subsubsection{Doppler Shift Velocity Measurement}

Researchers such as Cheng et al. (2010), Phillip \& Hanson (1991), Gieseller et al. (2007) and Lyle (2005) have demonstrated the ability to estimate the velocity of trace gases by measuring the Doppler shift created in the laser beam that traverses a flow (of an absorbent species). The general methodology, as seen in Figure 2.4, utilizes the differential Doppler shifted wavelength to quantify the bulk velocity. Other implementations of this measuring technique employ two laser beams, which penetrate the flow at different angles (relative to the direction of flow), resulting in Doppler shifted 
beams. However, to date this technology has only been applied to high speed flows because of the limits of the wavelength (and frequency) resolution of the detectors. As seen by the Doppler shift relation included in Figure 2.4, small velocities would create very small Doppler shifts in the absorption peak measurement. For velocities of $1 \mathrm{~m} / \mathrm{s}$ or less, depending on the wavelength, this shift is on the order of $1.135 \times 10^{-5} \mathrm{~nm}$. Accurately resolving subtle shifts of this magnitude challenges the limits of modern detector hardware. Additionally, when considering this technique and the full scale VOC mounted sensor, knowing the ideal location of the sensor would place it at the top rim (of the EFRT) or the breather vents (of the IFRT, Fixed Roof tanks and Horizontal tanks), there are additional challenges in creating sufficiently large angles between the differential beam setup without losing velocity/spatial resolution.

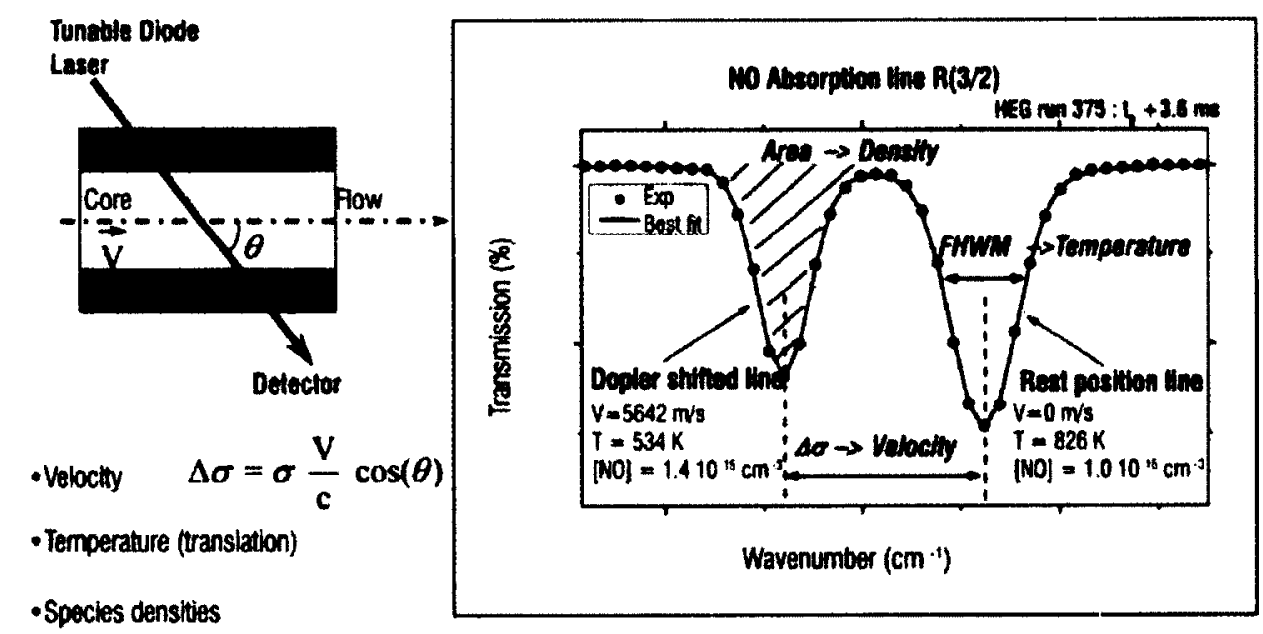

Figure 2.4: General methodology of Velocity and Density measuring techniques (Mohamed \& Lefebvre, 2009)

\subsubsection{Cross Correlation Velocity Measurement}

Cross-correlation velocity (CCV) measurement has been widely demonstrated by Herrmann et al. (1987), Cox et al. (1980), Morgan et al. (1968) and Rockwell et al. (2009) to provide precise measurements with good temporal resolution by using the time delay of a transient property (such as temperature) at two locations (vertically inline) to measure the velocity of the perturbations within the plume flow. In contrast to Doppler 
Shifted/WMS velocimetry, the technological and monetary investment required to perform this technique (computational/electronic) hardware is significantly reduced.

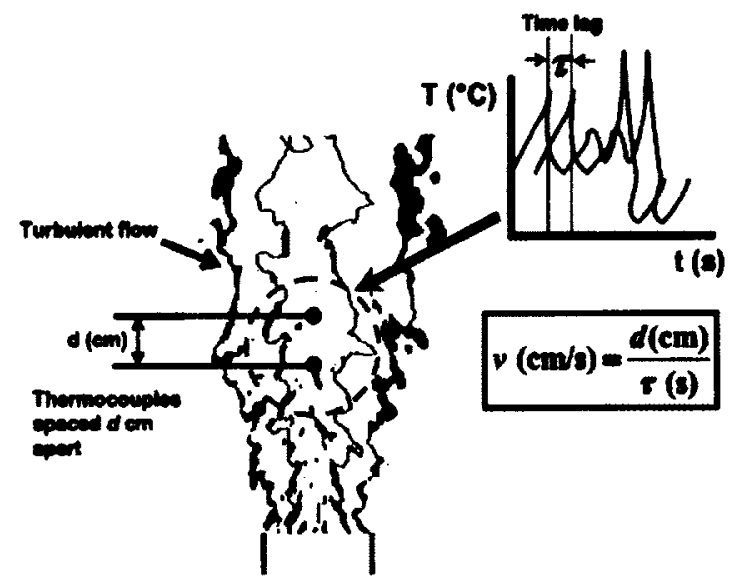

Figure 2.5: Example of measuring the velocity of a turbulent jet with a CCV Probe. Two thermocouples placed d (cm) apart (Rockwell, Rangwala, \& Klein, 2009)

\subsection{Selection of Absorption Spectroscopic and Velocity Techniques}

The possible methods of in-situ measurement of LST emissions vary with tank design and fall under two categories: vent-stack measurements and large surface area crosssection measurements. For fixed roof, internal floating roof and horizontal tanks, the evaporative losses flow through breather vents, which may be open or include a pressure/vacuum valve. Therefore, a sensor mounted on the vent stack could potentially be used to measure the hydrocarbon flux with either a single line or multi-line laserdetector setup. For EFRT, due the geometry of the storage tank, there is no vent stack to monitor and emissions are released non-homogeneously over the large cross-sectional area of the tank top. A sensor system to measure these emissions would necessarily be a multi-line (grid like) laser-detector implementation, creating an optical measurement plane over top of the tank shell. For a multi-line sensor implementation, the proven technique of tomographic reconstruction could be utilized to generate concentration maps of the evaporative losses (Wright et al., 2005). From tomographic studies, researchers such as Daun (2010), Pal et al. (2008), and Hindle et al. (2001) have demonstrated the ability of a 32- laser beam setup to provide sufficiently constrained data with which to 
reconstruct the spatially resolved concentration of the absorbing species. However, these studies were performed over relatively small control space (on the order of 50-84 millimetres) where the spatial resolution is documented to be inversely proportionally to the number of beams. This implies that a future full scale sensor would require a significant number of laser-detector pairs to provide reasonable spatial resolution above the EFRT. To satisfy these design criteria, DAS appears to be the most economical viable technique of the presented spectroscopic techniques. Considering the sizable monetary investment ( $\$ 50000$ and up) required for a laser-detector pair that utilizes WMS (required for Doppler shift velocimetry) with reasonable sensitivity, the economic feasibility of utilizing the Doppler shift technique is very poor for the single line setup let a alone the multi-line setup required for EFRTs.

\subsubsection{Selection of Absorption Spectroscopy Wavelength}

The design of a DAS system requires selection of an appropriate optical wavelength corresponding to the absorption region of the target species. From the DIAL study conducted by Chambers (2004), species-resolved fugitive emission concentrations were measured through canister sampling downwind of the storage tanks as tabulated in Table 2.1. From an overview of the field measurements of fugitive emissions, the lowest expected concentrations (i.e. values tabulated by Chambers), will be at minimum 2-3 times background ambient methane concentrations.

From a study conducted by Picard (2009), the composition of the evaporative losses from crude oil stock were ascertained from sampling the headspace of an EFRT, as plotted in Figure 2.6. The expected composition of evaporative losses from LSTs would have a similar molecular makeup as the gases in the vapour head space but concentration magnitudes in the fugitive emission plumes above the tank would be much lower. 
Table 2.1: Canister Sample from Condensate Tank Area at the Sour Gas Plant (Chambers, 2004)

\begin{tabular}{|l|l|r|r|}
\hline Compound Name & Formula & $\begin{array}{c}\text { Concentration } \\
(\mathbf{p p b})\end{array}$ & $\begin{array}{c}\text { Concentration } \\
\left(\mathbf{u g}^{3} / \mathbf{m}^{3}\right)\end{array}$ \\
\hline Methane & $\mathrm{CH} 4$ & $2.41 \mathrm{ppm}$ & 1576.45 \\
\hline C2 compounds & & $<50$ & \\
\hline C3 compounds & & $<50$ & \\
\hline Isobutane & $\mathrm{C} 4 \mathrm{H} 10$ & 2.98 & 7.07 \\
\hline Butane & $\mathrm{C} 4 \mathrm{H} 10$ & 11.73 & 27.81 \\
\hline Cyclopentane & $\mathrm{C} 5 \mathrm{H} 10$ & 1.49 & 4.26 \\
\hline Isopentane & $\mathrm{C} 5 \mathrm{H} 12$ & 4.01 & 11.79 \\
\hline Pentane & $\mathrm{C} 5 \mathrm{H} 12$ & 12.84 & 37.79 \\
\hline Isoprene & $\mathrm{C} 5 \mathrm{H} 8$ & 0.58 & 1.61 \\
\hline Cyclohexane & $\mathrm{C} 6 \mathrm{H} 12$ & 0.94 & 3.24 \\
\hline Methylcyclopentane & $\mathrm{C} 6 \mathrm{H} 12$ & 1.22 & 4.17 \\
\hline 3-Methylpentane & $\mathrm{C} 6 \mathrm{H} 14$ & 1.10 & 3.86 \\
\hline 2-Methylpentane & $\mathrm{C} 6 \mathrm{H} 14$ & 2.02 & 7.09 \\
\hline Hexane & $\mathrm{C} 6 \mathrm{H} 14$ & 6.70 & 23.55 \\
\hline Benzene & $\mathrm{C} 6 \mathrm{H} 6$ & 1.55 & 4.94 \\
\hline Cyclopentane, 1,3-dimethyl-, cis- & $\mathrm{C} 7 \mathrm{H} 14$ & 0.19 & 0.74 \\
\hline Cyclopentane, 1,2-dimethyl-, trans- & $\mathrm{C} 7 \mathrm{H} 14$ & 0.22 & 0.87 \\
\hline 1-Hexene, 5-methyl- & $\mathrm{C} 7 \mathrm{H} 14$ & 0.44 & 1.75 \\
\hline Methylcyclohexane & $\mathrm{C} 7 \mathrm{H} 14$ & 0.67 & 2.67 \\
\hline 2-Methylhexane & $\mathrm{C} 7 \mathrm{H} 16$ & 0.24 & 0.98 \\
\hline Heptane & $\mathrm{C} 7 \mathrm{H} 16$ & 0.85 & 3.48 \\
\hline Toluene & $\mathrm{C} 7 \mathrm{H} 8$ & 1.48 & 5.55 \\
\hline o-Xylene & $\mathrm{C} 8 \mathrm{H} 10$ & 0.20 & 0.86 \\
\hline m,p-Xylene & $\mathrm{C} 8 \mathrm{H} 10$ & 0.70 & 3.03 \\
\hline Cyclohexane, 1,3-dimethyl-, trans- & $\mathrm{C} 8 \mathrm{H} 16$ & 0.18 & 0.82 \\
\hline 2-Methylheptane & $\mathrm{C} 8 \mathrm{H} 18$ & 0.12 & 0.57 \\
\hline Hexane, 2,4-dimethyl- & $\mathrm{C} 8 \mathrm{H} 18$ & 0.20 & 0.95 \\
\hline Octane & $\mathrm{C} 8 \mathrm{H} 18$ & 0.31 & 1.44 \\
\hline Benzene, 1,2,4-trimethyl- & $\mathrm{C} 9 \mathrm{H} 12$ & 0.36 & 1.77 \\
\hline Nonanal & $\mathrm{C} 9 \mathrm{H} 180$ & 0.87 & 5.04 \\
\hline Decanal & $\mathrm{C} 10 \mathrm{H} 200$ & 1.02 & 6.50 \\
\hline & & & \\
\hline
\end{tabular}




\section{EFRT Headspace Vapour Composition}

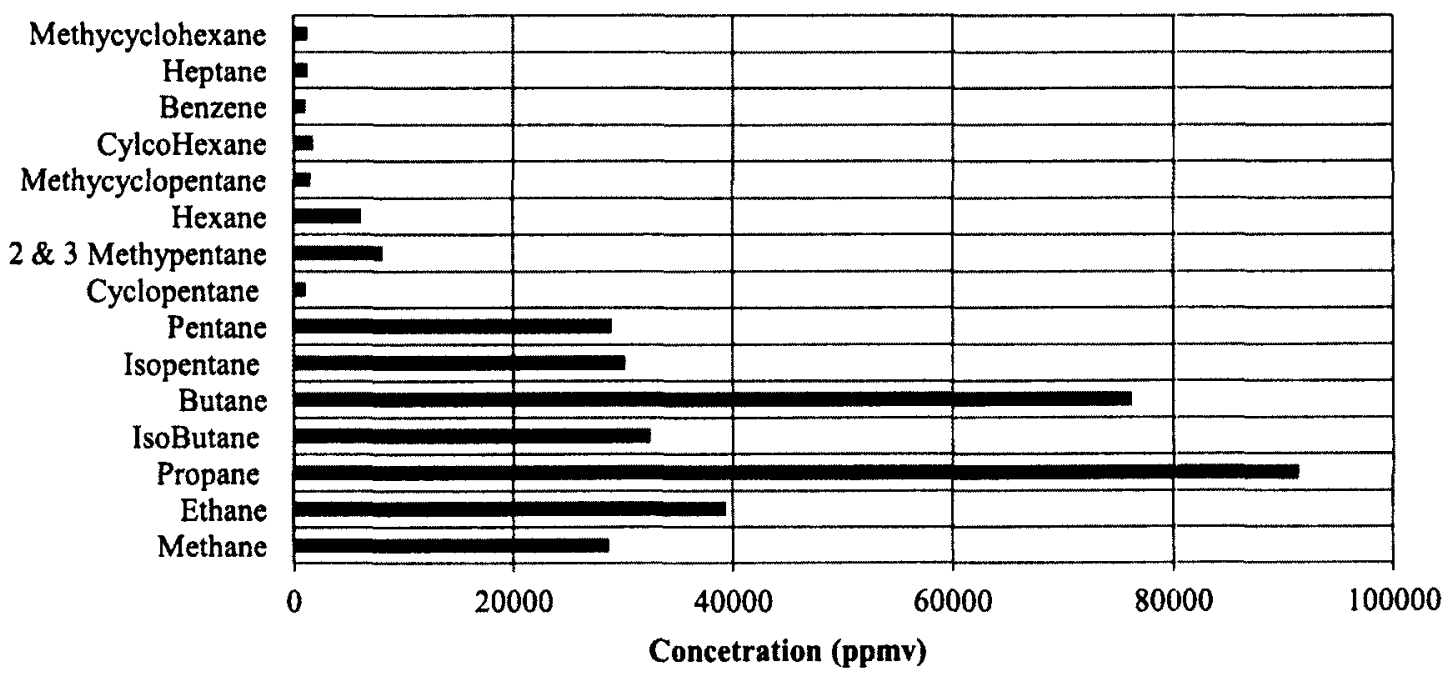

Figure 2.6: EFRT Headspace vapour composition and concentrations (Picard, 2009)

From the compositional breakdown of the fugitive emission surveyed at the tank site, the OSR for each molecule was found through an extensive survey of the following spectral databases.

- Aldrich Library of FTIR (Pouchert, 1981)

- $\quad$ AIST Spectral Database (AIST, 2010)

- $\quad$ HITRAN (HITRAN, 2008)

From the survey, it was ascertained that the OSRs for the range of anticipated hydrocarbon molecules lay within a wavenumber range of $2830-3000 \mathrm{~cm}^{-1}(3.125$ to $3.533 \mu \mathrm{m}$ ) (as tabulated in Table 2.2), with a secondary absorption spectral band around $1450 \mathrm{~cm}^{-1}(6.897 \mu \mathrm{m})$ (see Fig. 2.7 and 2.8 ). Formally known as the I and II fundamental Rotation-vibrational bands, these spectral ranges are the characteristic frequencies where the $-\mathrm{CH}_{3},-\mathrm{CH}_{2-}$, $\equiv \mathrm{C}-\mathrm{H}$ partial bonds and the $\mathrm{CH}_{4}$ molecule have their highest absorption strengths (HITRAN, 2008). In recent years, near-IR diagnostics that utilize the first overtone of the $\mathrm{C}$-H bond around the $6000 \mathrm{~cm}^{-1}$ have been preferred in "trace gas detection" research, primarily due to the wider availability and affordability of commercialized, high quality telecommunication diode lasers at these wavelengths. However, the near-IR absorption feature's relative strength is $\sim 100$ times lower than the 
Mid-IR's absorption feature, resulting in poor signal to noise ratios (Kllingbeil, 2007). Additionally, the expected atmospheric interferences that will absorb the electromagnetic radiation (mainly $\mathrm{H}_{2} \mathrm{O}$ ) plague the Near-IR region, requiring very high wavelength resolution from the Near-IR lasing source to selectively measure the hydrocarbon peak absorption without degradation from atmospheric absorption (HITRAN, 2008). Therefore, to design an economical VOC measurement system while maintaining good sensitivity, Mid-IR diagnostics will be explored.

Table 2.2: The Optimal Spectral Regions (OSR) for the VOC emissions from an EFRT (AIST, 2010; Pouchert, 1981)

\begin{tabular}{|c|c|c|c|c|c|}
\hline VOC & OSR $\left(\mathbf{c m}^{-1}\right)$ & OSR $(\boldsymbol{\mu m})$ & VOC & OSR $\left(\mathbf{c m}^{-1}\right)$ & OSR $(\boldsymbol{\mu m})$ \\
\hline Methane & $2850-3200$ & $3.125-3.508$ & 2-Methypentane & $2870-2990$ & $3.344-3.484$ \\
\hline Ethane & $2970-3000$ & $3.333-3.367$ & 3-Methypentane & $2850-2970$ & $3.367-3.509$ \\
\hline Butane & $2830-3020$ & $3.311-3.533$ & Hexane & $2840-3010$ & $3.322-3.521$ \\
\hline Isobutane & $2840-3010$ & $3.322-3.521$ & Cyclohexane & $2840-2970$ & $3.367-3.521$ \\
\hline Pentane & $2850-2970$ & $3.367-3.509$ & Methycyclohexane & $2830-2980$ & $3.355-3.533$ \\
\hline Isopentane & $2800-3000$ & $3.333-3.357$ & Benzene & $3000-3130$ & $3.194-3.333$ \\
\hline Cyclopentane & $2830-3020$ & $3.311-3.533$ & Heptane & $2840-3010$ & $3.322-3.521$ \\
\hline
\end{tabular}

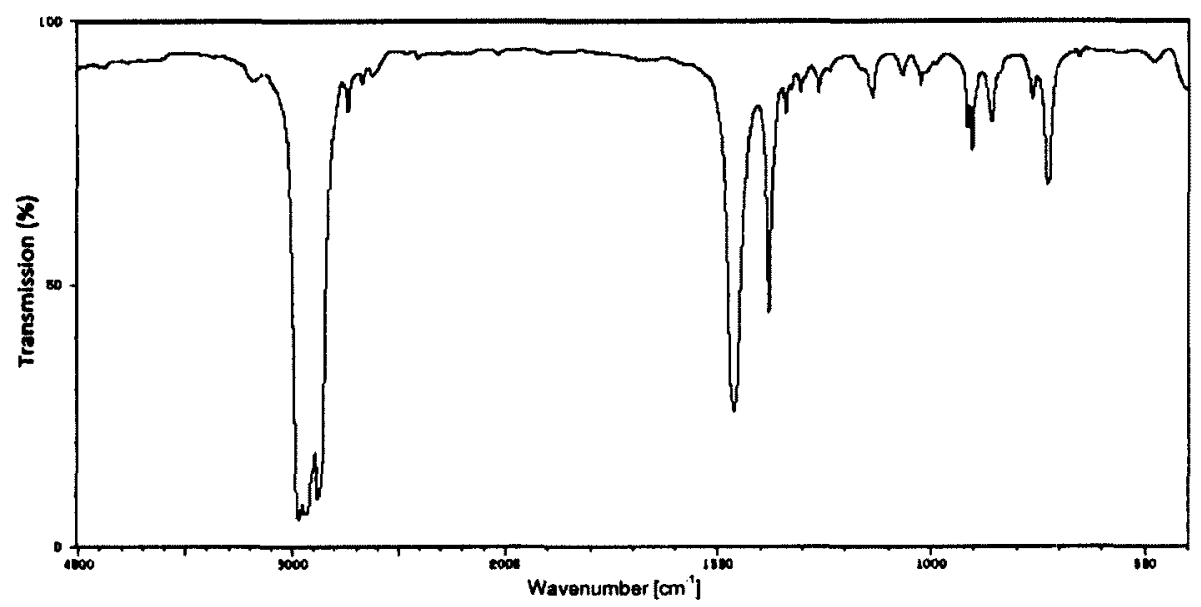

Figure 2.7: Transmission spectrum of Pentane (AIST, 2010) 


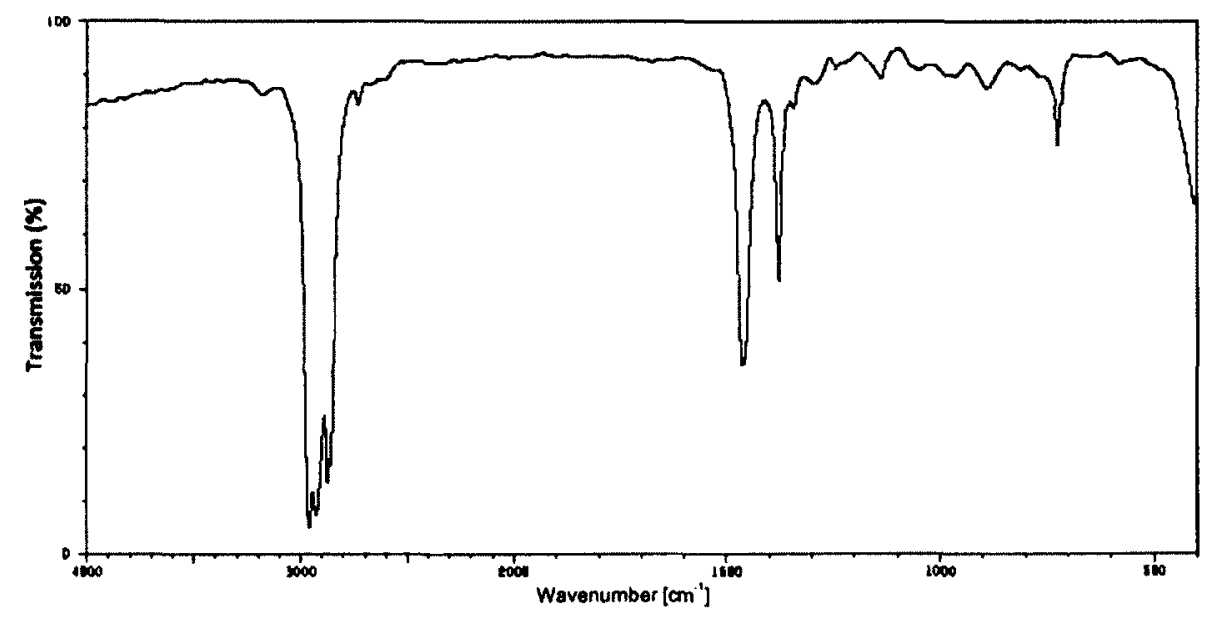

Figure 2.8: Transmission spectrum of Hexane (AIST, 2010)

\subsubsection{Mid-IR hydrocarbon selectivity}

The Mid-IR absorption spectroscopy provides the highest SNRs for hydrocarbon molecules. However, the OSR $\left(2850-3000 \mathrm{~cm}^{-1}\right)$ coresponding to the $\mathrm{C}-\mathrm{H}_{\mathrm{n}}$ bond is present in all hydrocarbon chains, such that multiple hydrocarbon absorption peaks overlap across this bandwidth. This leads to significant challenges in determining individual component concentrations from an absorption measurement for a multihydrocarbon mixture. Researchers such as Klingbeil (2007) have been able to use expected molecular composition to model and compare measured values of hydrocarbon mixtures (such as gasoline) with reasonable accuracy. However, the molecular composition of crude stock varies significantly as documented by Burruss \& Ryder (2003) which lowers the feasibility of using expected molecular compositions to measure mutlicomponent emission plumes. A potential solution for a field implementation would be to collect gas samples for speciation using a field deployable micro-gas chromatograph system in parallel with the emissions flux measurements of the laser absorption system. For the purposes of this thesis however, the speciation measurement problem was not considered further although it is recognized that this issue would need to be addressed prior to attempting full-scale measurements in a field setting. 


\section{Chapter 3}

\section{Background on MID-IR Absorption}

\section{Spectroscopy and Technology}

\subsection{Mid-IR Absorption Spectroscopy}

The Beer-Lambert law relates the absorption of electromagnetic radiation (at a known frequency) to the concentration of the species along the optical path as shown in Eq. 3.1 (Hollas, 2004),

$$
\alpha=-\ln \frac{1}{l_{o}}=-\ln \left(\tau_{R}\right)=\sigma(T, P, v) * C * L
$$

where:

$\tau_{R}$ is the transmittance of the target gas [-],

$\alpha$ is the absorbance of the target gas [-],

$I_{0}$ is the power of the incident radiation [W],

$I$ is the power of the transmitted radiation [W],

$\sigma(T, P, v)$ is the molar absorption coefficient as a function temperature, pressure and wavelength $\left[\mathrm{m}^{2} / \mathrm{mol}\right]$,

$C$ is the molar concentration of the target gas $\left[\mathrm{mol} / \mathrm{m}^{3}\right]$,

$L$ is the path length through the target gas traversed by the electromagnetic radiation $[\mathrm{m}]$,

$P$ is the barometric pressure $[\mathrm{Pa}]$

$v$ is the wavelength $[\mathrm{m}]$, and

$T$ is the temperature of the gas mixture $[\mathrm{K}]$. 
The Beer-Lambert law can be modified to measure the absorption of multi-component gases; the individual contributions are linearly cumulative as seen in Eq. 3.2 (Hollas, 2004; Kllingbeil, 2007).

$$
\alpha_{m i x}=-\ln \frac{I}{I_{o}}=-\ln \left(\tau_{R_{\text {mix }}}\right)=\sum_{i} \sigma_{i}(T, P, v) * C_{i} * L
$$

where:

$\tau_{R_{m i x}}$ is the transmittance of the multi-component gas mixture [-],

$\alpha_{m i x}$ is the absorbance measured of the multi-component gas mixture [-],

$\sigma_{i}(T, P, v)$ is molar absorption coefficient of the $\mathrm{i}^{\text {th }}$ gas as a function temperature, pressure and wavelength $\left[\mathrm{m}^{2} / \mathrm{mol}\right]$, and

$C_{i}$ is the molar concentration of the of the $\mathrm{i}^{\text {th }}$ gas $\left[\mathrm{mol} / \mathrm{m}^{3}\right]$.

The molar absorption coefficient varies with changes in gas sample temperature, barometric pressure, and the wavelength of electromagnetic radiation. While a change in pressure affects the peak strength of the molar absorption coefficient, the low variability of ambient pressure means that drastic changes to the molar absorption coefficient are not expected. Therefore, it is feasible to determine concentration by measuring the ambient pressure and directly interpolating between expected molar absorption constants calculated at different pressures (Hollas, 2004; D'Amato, 2006).

Figure 3.1 and Figure 3.2 show mid-IR absorption spectrum of methane near the target OSR $(3.392 \mu \mathrm{m})$ selected for the VOC sensor as a function of temperature and pressure. 


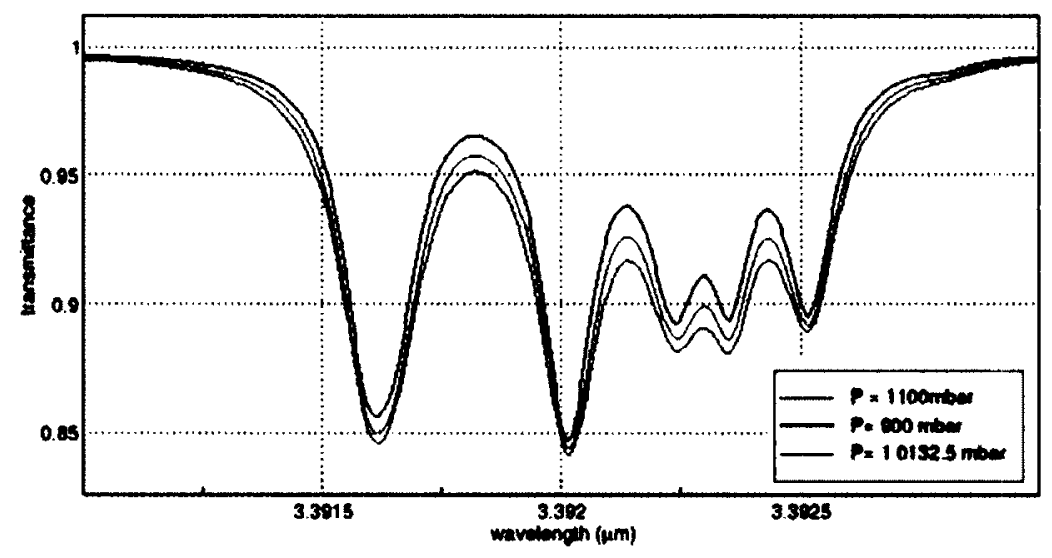

Figure 3.1: Variation of transmittance of $0.2 \%$ methane in air for different pressures at a fixed temperature of $300 \mathrm{~K}$ (Adapted from HITRAN 2008)

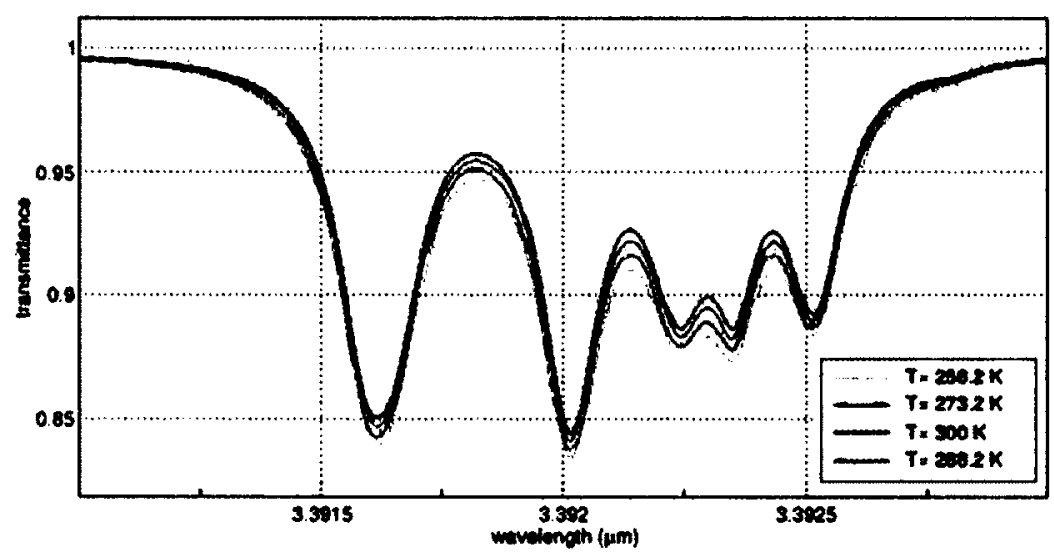

Figure 3.2: Variation of transmittance of $0.2 \%$ methane in air for different temperatures at a fixed pressure of $101.3 \mathrm{kPa}$ (Adapted from HITRAN 2008)

\subsubsection{Measurement of Volume Fraction using DAS}

By rearranging the Beer-Lambert relation from Eq. 3.1, it is possible to directly calculate the mean volume fraction of the target species (methane in this case) along the optical path as shown in Eq. 3.3,

$$
\chi_{C_{4}}=\frac{-R \cdot T}{P \cdot L \cdot \sigma\{T, P, v\}} \ln \left(\tau_{R}\right)
$$

where, $\chi_{\mathrm{CH}_{4}}$ is the measured volume fraction of methane averaged along the optical path, $L[\mathrm{~m}]$, and $R$ is the universal gas constant $[\mathrm{J} / \mathrm{K}-\mathrm{mol}]$. Because the instantaneous crosssection of the plume varies, it is generally more convenient to consider the product of the 
mean molar concentration along the optical path and the optical path length between the source and the detector as a single term. In this case, Eq. 3.1 may be conveniently expressed as

$$
C L_{n}=\frac{-1}{\sigma\{P, T, v\}} \ln \left(\tau_{R}\right)
$$

where $C L_{n}$ is the path integrated concentration $\left[\mathrm{mol} / \mathrm{m}^{2}\right]$ and the subscript $n$ identifies the relevant physical laser line (i.e. laser 1 or 2 in the present case). For the experiments presented in this thesis, the absorption cross-section strength was determined from the inlab calibration at the ambient temperature and pressure in the laboratory.

\subsubsection{Mid-IR DAS background interferences}

The natural logarithm of the fractional transmission of laser light, $\tau_{R}$, is a summation of the absorption strengths of all absorbing components of the gas mixture, including potential interfering species such as $\mathrm{H}_{2} \mathrm{O}, \mathrm{CO}_{2}$, and $\mathrm{CH}_{4}$ that are present in air. However, for the OSR centered at $3.392 \mu \mathrm{m}$, the contributions to the overall absorption from these background interferences is insignificant at anticipated concentrations as shown in Figure 3.3. Aside from ambient $\mathrm{CH}_{4}$ in the atmosphere (at typical volume fraction of $1.7 \times 10^{-6}$ ), the only contributor to background absorption is $\mathrm{H}_{2} \mathrm{O}$ at a typical volume fraction of $\mathrm{H}_{2} \mathrm{O}$ in the atmosphere of 0.0077 . While these interferences result in insignificant absorption, the variability of atmospheric background concentrations will correspond to a slight bias uncertainty. The measurement model is a relative measurement of the baseline $\left(I_{o}\right)$, taking into account the absorption caused by $\mathrm{H}_{2} \mathrm{O}$ and ambient $\mathrm{CH}_{4}$. However, if the ambient background concentrations varied significantly, this would result in a transient baseline and would introduce a variable bias uncertainty. For the in-lab setup this interference does not pose a significant problem due to relatively small laser path lengths (of $80 \mathrm{~cm}$ ) and atmospheric controlled environment, but might require further consideration for a future full scale sensor where laser path lengths would scale with the LST diameter $(\sim 5-90 \mathrm{~m})$. 


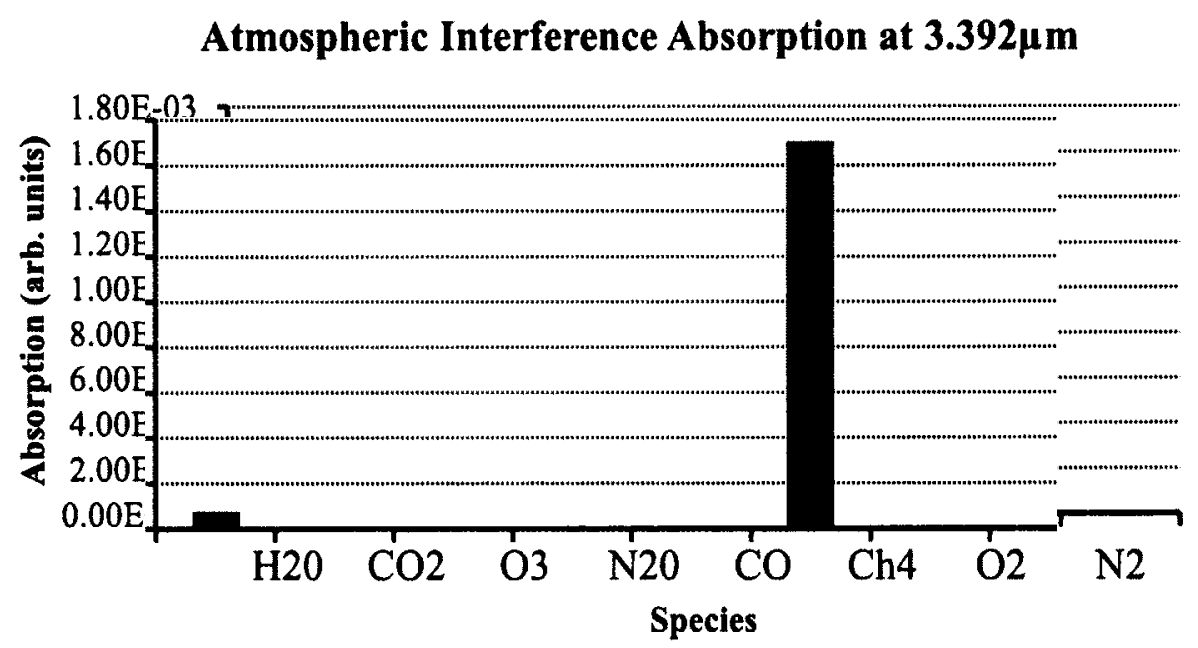

Figure 3.3: $\mathrm{A}$ breakdown of individual maximum atmospheric absorption across the OSR for an $80 \mathrm{~cm}$ path length at STP.

\subsubsection{Measurement of a Plume's Velocity through CCV with Mid-IR DAS}

Since the separation distance between the laser interrogation lines is fixed, by measuring the dominant time delay between fluctuations in the detector signal that correspond to movement of the eddies through the flow, the bulk flow velocity can be estimated. The time required for the turbulent eddy to pass between the laser lines is determined by cross-correlation of the time-resolved path-integrated concentration data obtained for the two, physically separated measurements lines. The cross-correlation coefficient is calculated according to Eq. 3.5,

$$
R_{x y}=\sum_{n=0}^{N-h-1} \frac{C L_{1}(n+h)}{\left[C L_{1}(n+h)\right]_{\text {Mean }}} \cdot \frac{C L_{2}(n)}{\left.\left[C L_{2}(n)\right)\right]_{\text {Mean }}} \quad 0 \leq h \leq N-1
$$

where:

$R_{x y}$ is the cross correlation parameter [-],

$C L_{1}$ is the path length integrated concentration of methane of the lower laser line $\left[\mathrm{mol} / \mathrm{m}^{2}\right]$,

$C L_{2}$ is the path length integrated concentration of methane of the upper laser line $\left[\mathrm{mol} / \mathrm{m}^{2}\right]$,

$h$ is the variable time lag (in samples) $[-]$, and

$N$ is the total number of discrete samples [-]. 
The time delay required for the flow to traverse the laser interrogation lines is measured by determining the sample offset, $h$, where the cross correlation function is maximized. The cross correlation parameter is calculated for the specified time period (corresponding to $N$ samples) for a range of time lags (corresponding to a range of sample offsets, $h$ ), to infer the time delay between the signals. An example of measuring the time delay using $\mathrm{CCV}$ can be seen below where two sample fluctuating signals (with no noise) are cross correlated to yield a distribution of possible time delays. As seen in the figure the cross-correlation function is maximized at the specified signal time delay of 2 seconds.

a)
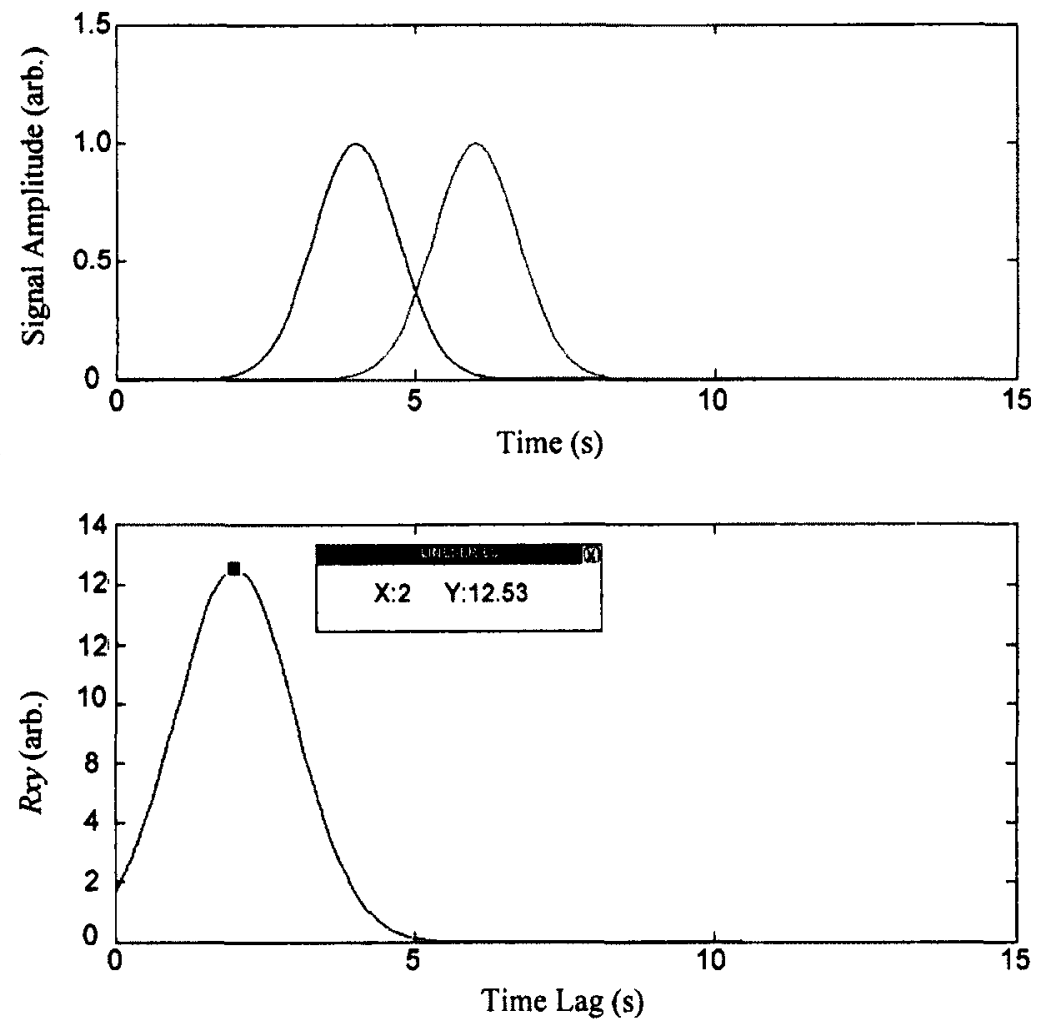

Figure 3.4: Example of CCV on plume fluctuation; (a) Example of two transient signals; (b) The Cross correlation parameter as a function of time delays, seen maximized at 2 seconds. 
The main parameters that affect the cross correlation function are the sampling frequency, sampling period, turbulent eddy behaviour, laser beam separation, measurement noise and the measurement signal's correlative properties (Rockwell et al. 2009). The sampling frequency determines the minimum turbulent fluctuation that can be resolved. In practice, the maximum sampling frequency is limited by the detector response time. To ensure proper transient resolution (i.e. no distortion due to low sample rates), a high sampling frequency is required, but the improvement in accuracy exponentially decays beyond a critical sampling frequency. The critical frequency is determined by the dynamics of the plume flow and the transient response of the detector. The sampling period must be long enough to identify a lag, but short enough so that the sensor can provide velocity readings at a reasonable rate. The turbulent eddy-size and the beam separation determine the sensor's ability to capture a time delay from a transient flow. For example, if the plume's eddy dissipation/generation is high and the beam spacing is relatively large, then the accuracy of the correlation function is reduced because there are less correlative properties within the signal. In order to maintain good correlative properties, the "frozen eddy" model must be satisfied, which states that the

eddy traversing through the measurement probes maintains its shape and size, thereby allowing robust correlation of the frozen eddy. Conversely, if the beam spacing is low such that the typical eddy size spans both detectors, the cross-correlation peak broadens and precision is reduced.

\subsubsection{Mid-IR Laser Source Survey}

To design a cost effective yet robust DAS system, it was critical to select an appropriate laser source. The following section briefly reviews currently available commercial mid-IR laser technologies and compares their relative advantages and disadvantages. Figure 3.5 as presented by Tittel et al. (2003) summarizes all the commericially avalible laser sources (over their operating wavelength). The fundamental rotational-vibrational bands that represent the OSRs of hydrocarbons are highligted in blue. The following sections summarizes the technology of the most prominentmid-IR laser sources and their corresponding advantages and disadvantages. 


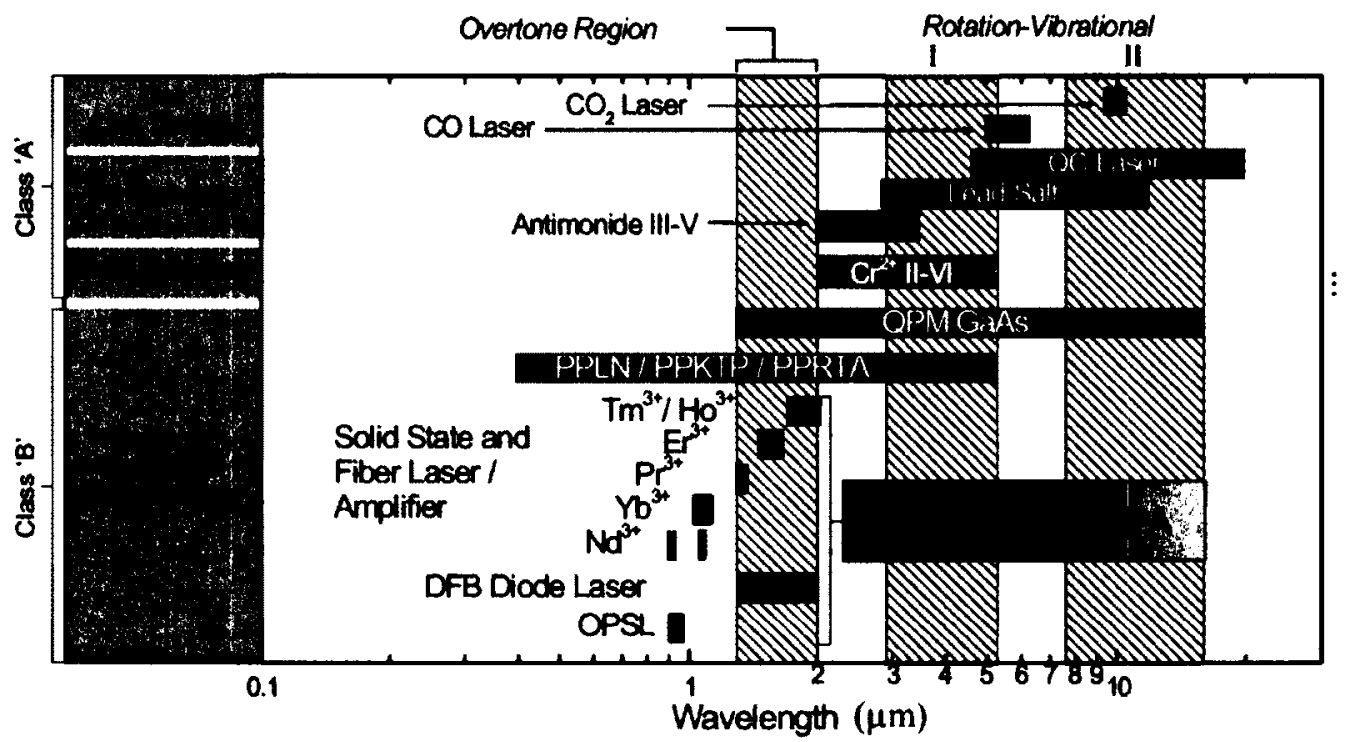

Figure 3.5: Mid-IR Spectroscopic Sources and their operating ranges (Tittel et al., 2003)

\subsubsection{Helium Neon Lasers}

First demonstrated by Chu \& Hogg (1966) in a study to quantify the absorption of atmospheric background concentrations of methane, the Helium Neon ( $\mathrm{HeNe})$ laser has become a valuable tool for spectral measurements. Grant (1986) demonstrated the sensitivity of $\mathrm{HeNe}$ based measurements, producing a trace gas measurement system that delivered a SNR of 200, an equivalent sensitivity (i.e. the detectivity limit) of $3 \mathrm{ppm}$. McManus et al. (1989) demonstrated a measurement system that employed a Zeemansplit HeNe laser to deliver SNR $\sim 5 \times 10^{5}$, an equivalent sensitivity of $20 \mathrm{ppb}$. The emission of HeNe lasers in the $3.39 \mu \mathrm{m}$ region of the infrared makes it especially useful for probing spectroscopic absorption of hydrocarbons. Klingbeil et al. (2006) published spectroscopic data and temperature/pressure dependencies for a wide variety of hydrocarbons and multi-component fuel tested using a HeNe laser.

The makeup of a typical HeNe laser can be seen below in Figure 3.6. The gain medium of the laser is a mixture of helium and neon held at a constant molar ratio at low pressure. Excitation comes from a high-voltage, typically DC electrical discharge between an anode and cathode. The photon pathway is held between a reflector and an output coupler with a transmission of 1 percent as specified by Thorlabs (2011). The typical wavelengths achieved by $\mathrm{HeNe}$ gas lasers are $0.543,0.594,0.633,1.150,1.520$ 
and $3392 \mu \mathrm{m}$. As shown in Figure 3.5 , the $3.392 \mu \mathrm{m}$ band overlaps the fundamental (rotational-vibrational) absorption region of hydrocarbons, which has the capacity to provide the highest signal to noise ratios in absorption spectroscopy. However, these lasers suffer from noise intensities of $1-5 \%$, requiring additional computational effort in signal processing or addition of reference beams for noise cancelation (Mielenz \& Nefflen, 1965; Kllingbeil, 2007). In addition, due the narrow bandwidth of the gain medium, these lasers generally only offer single wavelength operation.

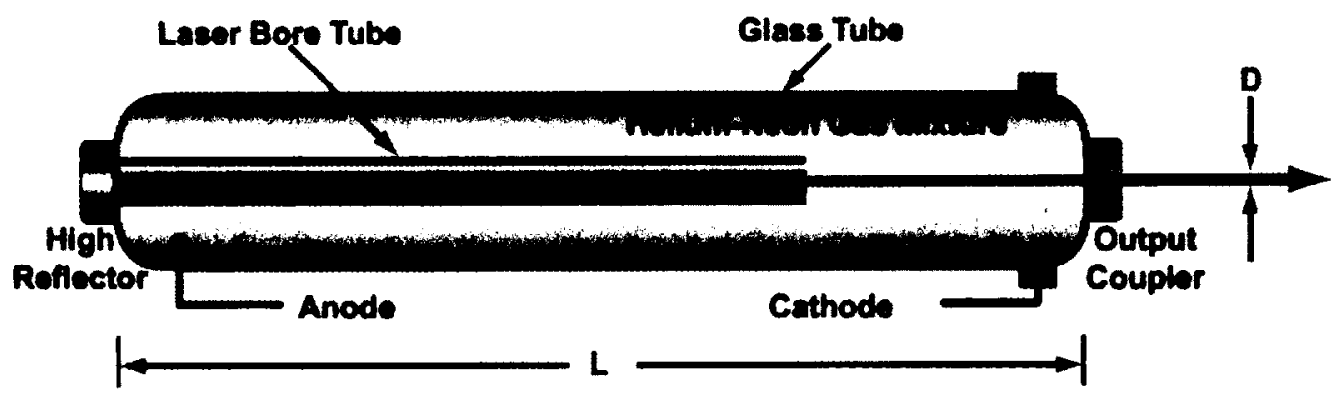

Figure 3.6: Schematic of HeNe laser (Thorlabs, 2011)

\subsubsection{Lead Salt Diode Lasers}

Lead salt lasers have been used for the last 40 years in a variety of applications, such as pollution monitoring, medical diagnostics, and space applications (Springholz et al., 2006). Their popularity in diagnostics is mainly due to the fact that they cover such a wide spectral range of 3 to $30 \mu \mathrm{m}$ and outperform (only) other band gap lasers due to their relatively higher operating temperatures (Springholz et al., 2006). Lubken et al. (1999) demonstrated the utility of lead salt laser when constructing the first rocket-borne TDL spectrometer to conduct in-situ trace gas measurement in the middle atmosphere with sensitivities ranging from $10^{-4}-10^{-5}$. In addition to atmospheric trace gas analysis, lead salt lasers have been demonstrated in medically motivated trace gas analysis. Lachish et al. (1987) developed a lead salt laser-based spectroscopic measurement system that measured levels of ammonia exhaled in human breath with 1ppm accuracy. Skeldon et al. (2006) similarly used lead salt lasers to quantify ethane in human breath (a potential marker for disease) with $0.1 \mathrm{ppb}$ accuracy. 
The lead salt laser is comprised of a single crystal of semiconductor material that is shaped into an optical cavity (Tittel et al., 2003). These materials are generally $\mathrm{PbTe}$, $\mathrm{PbSe}$ and $\mathrm{PbS}$ and various alloys of these compounds. To achieve lasing action, a forward bias current is applied to the $p-n$ junction of the semiconductor, which populates the nearly empty conduction band, which in turn stimulates emission across the band gap (between the conduction and nearly full valence band). Tunability can be achieved by varying the device temperature or the injection current. The wide tunability and narrow line width are some of the major advantages of the lead salt laser (Springholz et al., 2006). While many modifications have been made over the years to improve the beam spatial quality (such as the addition of $\mathrm{PbEuSeTe}$ or $\mathrm{PbSnTe}$ layers), the main disadvantage of the lead salt laser lies in the required operating temperatures of below $200 \mathrm{~K}$ in continuous wave mode, limiting the applicability of this laser in the field (Tittel et al., 2003).

\subsubsection{Quantum Cascade Lasers}

While Quantum Cascade Laser (QCL) technology is only a decade old, it has been noted as a revolutionary mid-IR source of unprecedented sophistication. Recent notable applications include the HIAPER Pole-to-Pole Observations (HIPPO) experiment, where trace gases (more specifically $\mathrm{CO}_{2}, \mathrm{CH}_{4}, \mathrm{CO}, \mathrm{N}_{2} \mathrm{O}$ ) in the atmosphere were measured pole to pole over the Pacific/ North America at sub ppb accuracy. This study indicated several keys areas of pollution, and provided data that demonstrated global atmospheric transport modes and their driving mechanisms (Wofsy, 2011). QCL technology has also been utilized in the medical field to improve the trace gas analysis of human breath, as demonstrated by Shorter et al. (2010), where a fully automated QCL spectrometer was employed to deliver sub-ppb accuracy of trace gases exhaled through human breath.

While semiconductor lasers employ the band gap between the conduction and valence bands, QCL's employ the subbands that exist within the conduction band quantum wells (Cockburn, 2006). The nature of this configuration limits radiative losses and allows for significant optical gains, providing key advantages over conventional MidIR diode lasers (Cockburn, 2006). Tunability of a QCL is achieved by varying its 
operating temperature. By altering the temperature set point of the QCL chip's heat sink or by altering the QCL's injection current (which induces an even faster response), it is possible to efficiently and rapidly vary the QCL output wavelength. The capability of fast wavelength alteration allows this technology to be easily implemented in absorption spectroscopy using modulation techniques (Kosterev et al., 2008). Even though QCLs have been found to be significantly superior to traditional semiconducting lasers, their required operating temperatures still require cryogenic cooling which limits their field applicability (Tittel et al., 2003).

\subsubsection{Difference Frequency Generation (DFG) Laser}

Another approach in achieving lasing sources in the mid-IR region is the nonlinear frequency mixing conversion process known as Difference Frequency Generation (DFG). DFG technology has been well demonstrated in mid-IR spectroscopy of hydrocarbons in ambient conditions by researchers such as Tittel et al. (1999), Töpfer et al. (1997), and Armstrong et al. (2010), by achieving spectroscopic measurements with the lower detection limits of $15 \mathrm{ppb}, 1 \mathrm{ppb}$ and $54 \mathrm{ppm}$ respectively.

The technique involves using two near-IR laser sources at different frequencies and a unique crystal to mix the frequencies to produce the desired Mid-IR wavelength (known as the "idler") (Tittel et al. 2003). Generally the crystal used in the conversion process is made of Periodically Poled Lithium Niobate (PPLN). The use of a PPLN as the nonlinear mixer allows for the use of near-IR sources instead of much larger laser sources such as Ti:Sahpphire laser sources. A general makeup of a DFG source can be seen in (Tittel et al., 2003; Kllingbeil, 2007).

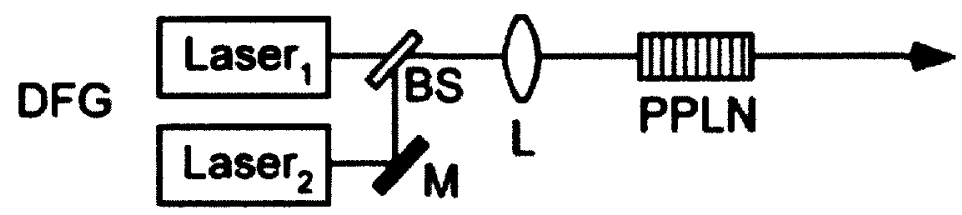

Figure 3.7: Schematic of DFG laser source (Tittel, Richter, \& Fried, 2003) 
Tunability of DFG laser sources is achieved by tuning either the "pump" laser (highest frequency) or the "signal" laser (lower frequency) or simultaneously tuning both laser sources. The main operating condition of the DFG is that all three light waves lie in phase, which is most often satisfied by using the refractive indices of each wave through the nonlinear mixing crystal. If three waves are not in-phase, they will not propagate in the same direction and instead bleed off, limiting the overlap region and the DFG power (Tittel et al., 2003). To ensure correct phase, the refractive indices must be tuned as the difference frequency is tuned. This can be achieved by altering the temperature of the crystal or by tuning the pump and signal simultaneously. This limits the use of DFG in harsh environments, due to the temperature effects on the PPLN crystal (Tittel, Richter, \& Fried, 2003; Klingbeil, 2007).

\subsubsection{Optical Parametric Oscillators (OPO)}

Optical Parametric Oscillator (OPO) sources employ parametric amplification through a nonlinear crystal to achieve lasing action at the Mid-IR range (Tittel et al., 2003). OPOs have the advantage of being able to generate two output beams (signal ( $v 1)$ and idler (v2)) from a single laser source (operating at a frequency $v 3$ ) under the energy conservation principle $(v 1+v 2=v 3)$. The frequency of the output beams is determined by a phase matching criteria, which can be varied over wide ranges. This is seen to be a significant advantage because it allows access to the mid-IR, far-IR and terahertz spectral region which is otherwise difficult to achieve while maintaining such wide wavelength tunability. OPOs have also been utilized in spectroscopy trace gas measurement systems to produce capable sensors that have very low detection limits, as demonstrated by Kühnemann et al (2004) who used an OPO-based spectroscopic sensor to measure ethane at sub-ppt levels.

The OPO system is generally comprised of a near-IR laser source and a single resonant cavity, as shown in Figure 3.8 (Tittel et al, 2003). Within the cavity, a resonant light circuit is created through the implementation of a series of mirror (M) and semitransparent mirrors (SM) in order to force laser emission continuously through the PPLN to achieve higher quality of the output beams. Tunability is achieved with a piezo 
driven cavity mirror that alters the phase, thereby changing the output beam frequencies. An étalon (ET) is synchronously tuned to maintain single mode operation. As mentioned earlier the use of PPLN crystal limits the field implementation due to adverse control effects of temperature variations (Pashotta, 2008; Tittel, Richter, \& Fried, 2003).

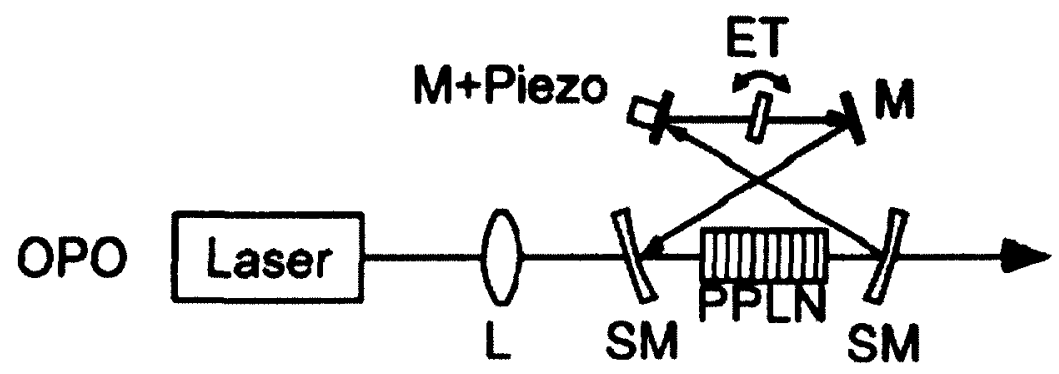

Figure 3.8: Optical Parametric Oscillation Lasing Methodology (Tittel et al., 2003)

\subsubsection{Motivation and Selection of the Mid IR Lasing Source}

Table 3.1 summarizes key observations from the brief survey of potential laser sources presented above. For the envisioned application to measure VOC emissions from EFRTs, the selected mid-IR lasing source requires low manufacturing cost coupled with the versatility to be deployed in the field. As discussed earlier in Section 2.3, the future ideal sensor layout would likely require greater than 32 laser detector pairs (potentially up to 64 if cross-correlative techniques are implemented) to create a bounded measurement of the hydrocarbon mass flux emanating from the EFRT. Therefore, the design of the in-Lab testing apparatus must take into account the cost per laser interrogation line and field implementabilty as dominant selection criteria.

The HeNe laser was selected as the optical sensor's lasing source for both the inlab testing apparatus and (if justified by the former) the future full scale sensor. The large power output of the HeNe allows for the construction of multiple interrogation laser lines per source. Its operating requirements also allow for easier field implementation when comparing to the other commercially available lasing sources, which require cryogenic cooling and controlled environments. 
Table 3.1: Summary of Commercially Available Mid-IR Laser Source Survey (Pashotta, 2008; Tittel, Richter, \& Fried, 2003; Klingbeil, 2007; Cockburn, 2006;

Kosterev, et al., 2008; Springholz, Schwarzl, \& Heiss, 2006; Weber, 1999)

\begin{tabular}{|l|c|c|c|c|c|c|c|}
\hline Type & $\begin{array}{c}\text { Wavelength } \\
(\mu \mathrm{m})\end{array}$ & $\begin{array}{c}\text { Tunability } \\
\left(\mathbf{c m}^{-1}\right)\end{array}$ & $\begin{array}{c}\text { Power } \\
(\mathbf{m W})\end{array}$ & Linewidth & $\begin{array}{c}\text { Beam } \\
\text { Profile }\end{array}$ & $\begin{array}{c}\text { Operating } \\
\text { Requirements }\end{array}$ & Cost \\
\hline HeNe & $\begin{array}{c}0.543,0.594, \\
0.633,1.150, \\
1.5203 .392\end{array}$ & None & $0.5-50$ & $\begin{array}{c}200 \mathrm{MHz} \text { to } \\
1.5 \mathrm{GHz}\end{array}$ & $\begin{array}{c}\text { Divergent } \\
\text { Tem00 }\end{array}$ & -- & $\$$ \\
\hline Pbsalt & $3-30$ & 100 & $0.1-0.5$ & $1-1000 \mathrm{MHz}$ & $\begin{array}{c}\text { Elliptical } \\
\text { Highly } \\
\text { astigmatic } \\
\text { and } \\
\text { divergent }\end{array}$ & $\begin{array}{c}\text { Cryogenic } \\
\text { cooling }\end{array}$ & $\$ \$$ \\
\hline QCL & $3.5-24$ & 35 & $1-100$ & $0.001-$ & $\begin{array}{c}\text { Elliptical } \\
\text { astigmatic } \\
\text { highly } \\
\text { divergent }\end{array}$ & $\begin{array}{c}\text { Cryogenic } \\
\text { cooling } \\
\text { Low noise } \\
\text { driving } \\
\text { electronics }\end{array}$ & $\$ \$ \$$ \\
\hline DFG & $2.3-4.6$ & 15 & $0.1-1$ & $1 \mathrm{MHz}$ & $\begin{array}{c}\text { Near } \\
\text { Gaussian }\end{array}$ & $\begin{array}{c}\text { Telecom } \\
\text { Env. }\end{array}$ & $\$ \$ \$ \$$ \\
\hline OPO & $2.3-4$ & 1900 & $10-100$ & $0.15 \mathrm{MHz}$ & Tem00 & $\begin{array}{c}\text { Low noise } \\
\text { environment }\end{array}$ & $\$ \$ \$ \$$ \\
\hline
\end{tabular}

\subsection{Mid-IR Detectors}

Mid-IR detectors are classified into two main groups, photoconductive (PC) and photovoltaic (PV) detectors, which are differentiated based on the conversion process of received photons to a measureable output signal (Dereniak \& Boreman, 1996; Kllingbeil, 2007). The PC detector is comprised of a semi-conductive material as the detection element whose electrical resistance is a function of the amount of incident light striking the element. The semi-conductive material band-gap energy level (as discussed in Section 3.1.5) determines the threshold wavelength that the element can detect. If the incident radiation energy is higher than the band-gap energy level, the current produced will be proportional to the number of incident photons. Therefore, for a fixed-power lasing source, the detection sensitivity varies linearly with wavelength and will drop significantly once the band-gap energy threshold is reached (Dereniak \& Boreman, 1996; Klingbeil, 2007). 
PV detectors similarly generate a current or voltage that is proportional to the amount of incident radiation on the detector element and are also limited by a semiconductive threshold that is determined by the semi-conductors band-gap energy level. The key advantage of PV detectors is that, unlike PC detectors, they are not prone to $1 / \mathrm{f}$ noise (Dereniak \& Boreman, 1996; Klingbeil, 2007). 1/f noise (also known as pink noise) is a noise profile that has a power spectral density that is inversely related to the frequency. Since $1 / \mathrm{f}$ noise levels are inversely proportional to frequency, at low frequencies the Signal to Noise Ratio (SNR) is severely affected. However, if the overall amplitude of the signal is sufficiently higher than the expected noise levels, a PC detector is the more cost-effective choice

To determine the most appropriate Mid-IR detector selection, the operational wavelength, frequency bandwidth, noise characteristics and cost needed to be considered. The operational wavelength and frequency bandwidth for commercially available detectors is summarized in Figure 3.9 (Klingbeil, 2007). These plots suggest that the $\mathrm{InSb}, \mathrm{HgCdTe}, \mathrm{PbSe}$ detectors are candidates for the proposed Mid-IR optical sensor system and have good working frequency response bandwidths $(\sim 1 \mathrm{MHz})$.
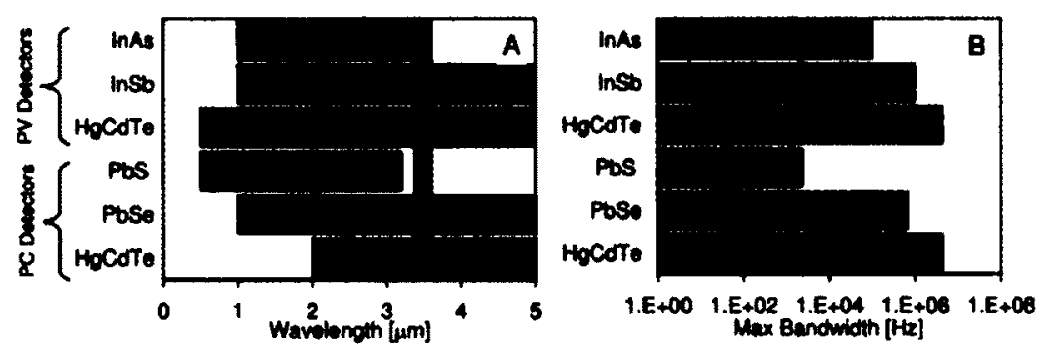

Figure 3.9: The Operation Wavelength (A) and the Frequency Bandwidth (B) of commercially available Mid-IR detectors; the Blue region indicates (see $A$ ) the operational wavelength of interest for VOCs (Klingbeil, 2007)

To characterize the noise from each detector, the detectivity $\left(D^{*}\right)$ needs to be quantified, where $D^{*}$ is defined as:

$$
D^{*}=\frac{\sqrt{A_{D} \cdot \Delta f}}{N E P}
$$

where:

$$
D^{*} \text { is the detectivity }\left[\mathrm{cm}-\mathrm{Hz} \mathrm{z}^{1 / 2}-\mathrm{W}^{-1}\right] \text {, }
$$


$A_{D}$ is the active detector area $\left[\mathrm{cm}^{2}\right]$,

NEP is the noise equivalent power [W], and

$\Delta f$ is the frequency bandwidth of the detector $[\mathrm{Hz}]$; determined by

$$
\Delta f=\frac{1}{2 \pi \tau}
$$

where:

$\tau$ is the response time of the detector $[\mathrm{s}]$

In addition, the Signal to Noise Ratio (SNR) of the detector is determined by the following relation:

$$
S N R=\frac{P_{I}}{N E P}=\frac{P_{I^{\prime} \cdot D^{*}}}{\sqrt{A_{D^{*}} \cdot \Delta f}}
$$

where:

$P_{I}$ is the power of the incident radiation $[\mathrm{W}]$, and $N E P$ is the Noise Equivalent Power [W].

The detectivity of commercially available detectors as a function of operating wavelength and temperature is summarized in Figure 3.9 (Piotrowski \& Rogalsk, 2007). The plotted detectivity curves illustrate that for the given Mid-IR wavelength bandwidth, the highest detectivity detectors (InAs, InSb) require low operating temperatures, generally achieved though cryogenic cooling or by using 3 stage thermoelectric heat sinks. This adds to the operational cost and limits their field applicability (Dereniak \& Boreman, 1996)

Eq. 3.8 shows that to achieve good SNRs, it is important to have high detectivity values. However, the detector selection must primarily satisfy the cost and room temperature operating criteria. The future full-scale sensor will require on the order of 32 laser line-detector pairs and while the ample power of the HeNe laser provides the capability to create multiple laser lines per lasing source, the manufacturing cost of the future full scale sensor will grow proportional to the number of detectors. 


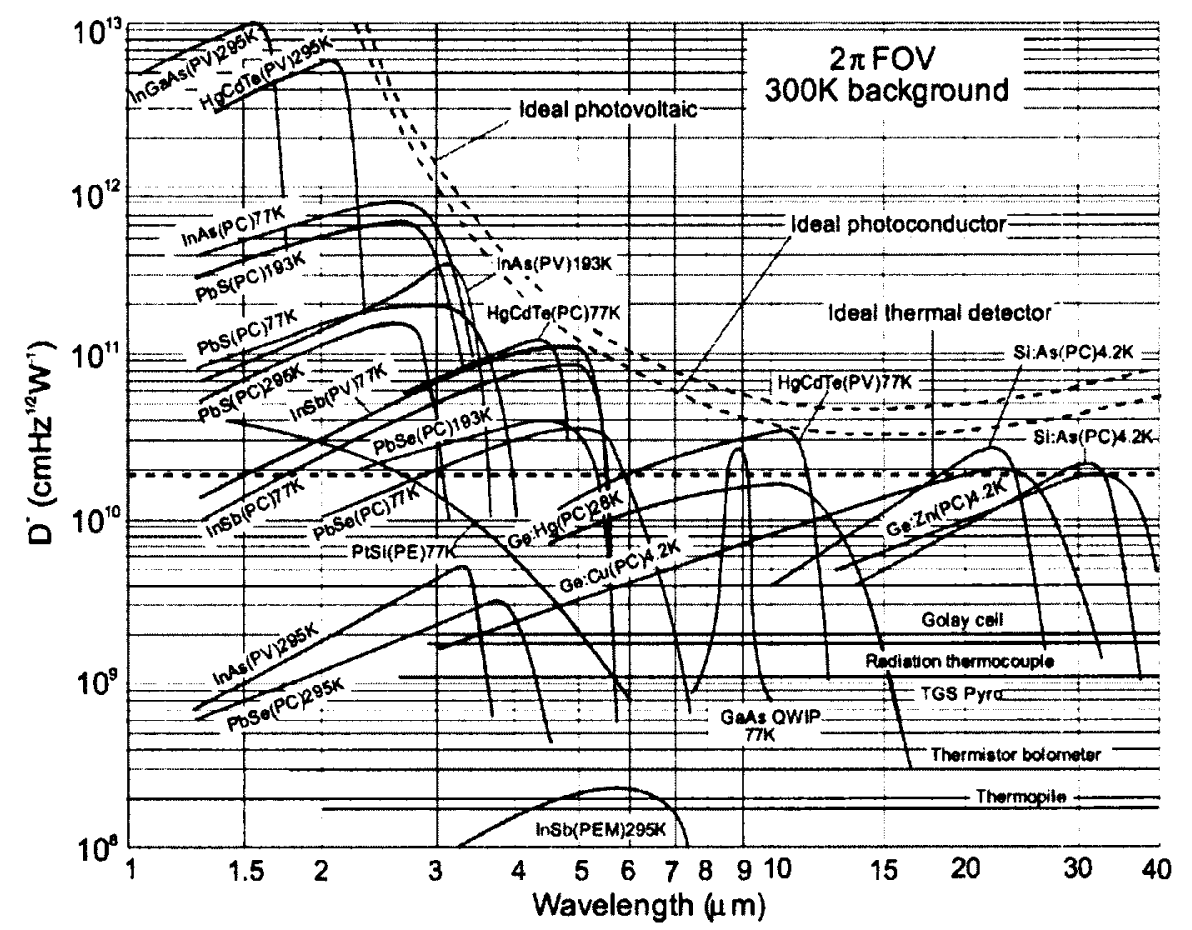

Figure 3.10: Comparison of the $D^{*}$ of various commercially available IR detectors (Piotrowski \& Rogalsk, 2007)

\subsubsection{Motivation and Selection of the Mid IR detector}

The preferred choice to satisfy the cost and operating temperature criteria is a Mid-IR $\mathrm{PbSe}$ detector, which costs in the range of $\$ 300-400$ (Thorlabs, 2011). However, the $\mathrm{PbSe}$ detectors commercially available were PC detectors, only suitable for high frequency applications due to their $1 / \mathrm{f}$ noise characteristics that degrade the lower frequency bandwidth severely. For spectroscopic sensors employing low WMS (higher that $1 / \mathrm{f}$ degraded bandwidth, but lower that the max response frequency of the detector), $\mathrm{PbSe}$ detectors could provide a cost effective detector selection while still providing reasonably good detectivity.

The alternate detector choice that satisfies the cost and operating temperature criteria was the HgCdTe detector. From the detector survey, a HgCdTe detector that provided good detectivity while operating at room temperature was found to require roughly double the monetary investment (\$800-900) (Viggo System, 2011). However, 
PV HgCdTe detectors provide significantly better noise characteristics (with respect to frequency), allowing implementation for both DC and high frequency sensor applications. The DC (and low frequency) noise performance allows for continuous wave operation eliminating the need for noise compensation techniques (like optical chopping or modulation) which allow the signal of interest to be sensed as a higher frequency signal (which generally has better noise).

The theoretical SNRs for a $10 \mathrm{~cm}$ plume (of $300 \mathrm{ppm}$ methane in air) with $10 \%$ fluctuations (tabulated in Table 3.2) as illustrated in Figure 3.11, inferred a lower detection limit (for detector noise only) of $0.1 \mathrm{ppb}$ at room temperature, making the PV $\mathrm{HgCdTe}$ the ideal candidate for proposed sensing application. It should be noted that the DC component represents the average (steady) absorption signal and $\mathrm{AC}$ component represents the transient fluctuations in path length in the flow.

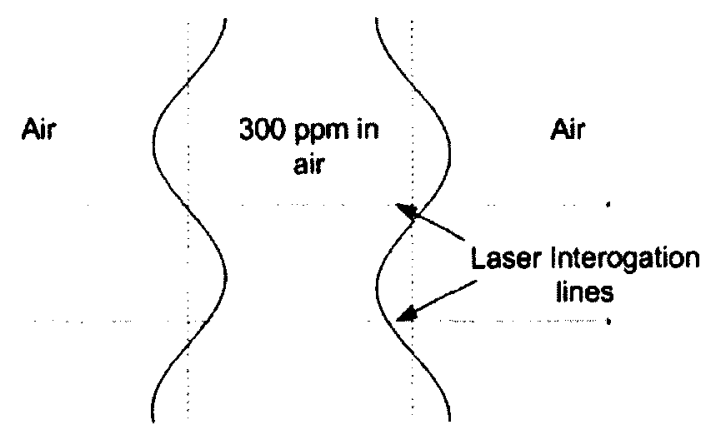

Figure 3.11: Schematic of simplified plume

Table 3.2: Theoretical SNR's for PV HgCgTe

\begin{tabular}{|c|c|c|}
\hline & SNR (DC) & SNR (AC) \\
\hline Max & 3177.5 & 313.3 \\
\hline Min & 2210.4 & 220.3 \\
\hline
\end{tabular}




\section{Chapter 4}

\section{Experimental Setup}

\subsection{General Approach}

To research the potential of the Mid-IR CCV technique measurement system, a simplified experiment was designed that comprised a pair of offset laser lines and detectors displaced vertically above an $50.4 \mathrm{ID} \mathrm{mm}$ diameter test plume, as shown schematically in Figure 4.1. This setup provided a means of developing and validating the experimental technique to determine the response and accuracy of the proposed sensor arrangement.

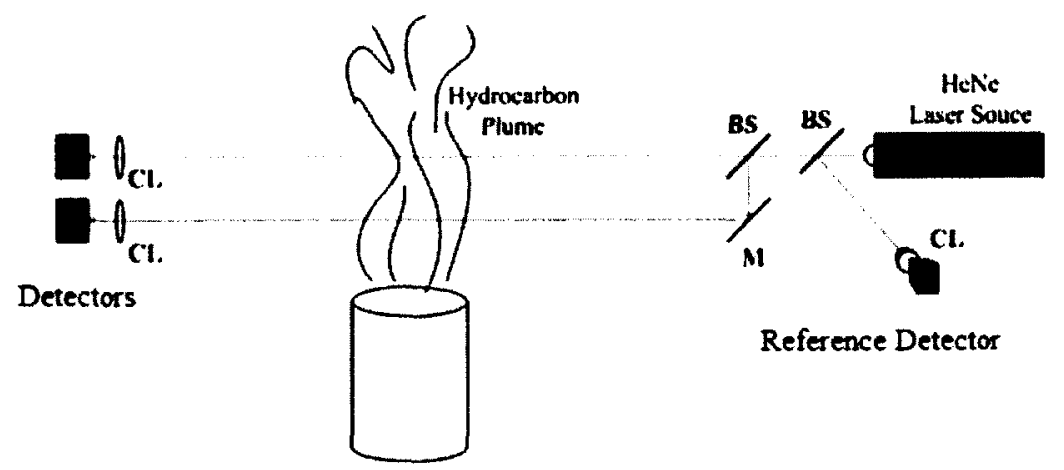

Figure 4.1: Schematic of Proposed Experimental Apparatus to Measure a Line Flux through cross-correlation techniques; BS - Beam splitter, M - Turning mirror, CL Collimating lens

Because the instantaneous laser path length varies within the unconfined hydrocarbon plume, a secondary experimental setup using an IR spectroscopic flow through cell in place of the plume generator was used to quantify sensitivity and accuracy of the system for concentration measurements, as shown schematically in Figure 4.2. This fixed the path length of the laser through the absorbing gas medium allowing quantitative comparison between the known concentration in the flow-through cell and the measured concentration from the laser system. 


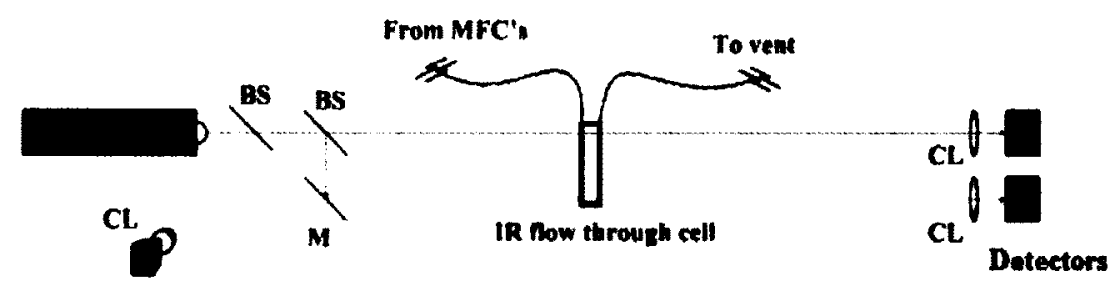

Refereace

Detector

Figure 4.2: Schematic of proposed experimental apparatus to determine concentration sensitivity; BS - Beam splitter, M - Turning mirror, CS - Collimating lens

In the following sections, the design and construction of the experimental apparatus is broken down into three main sections: the VOC optical measurement system, the driving electronic/computational hardware, and the Schlieren imaging system used to track the buoyant plume.

\subsection{VOC Measurement System}

The VOC measurement system includes three main components: the laser emission apparatus, the detector assembly, and the plume generator, as shown in the Figure 4.3. The entire apparatus was set up on an optical granite table manufactured by Tru Stone Corp. (S/N 30737), to aid optical alignment and reduce vibrations.

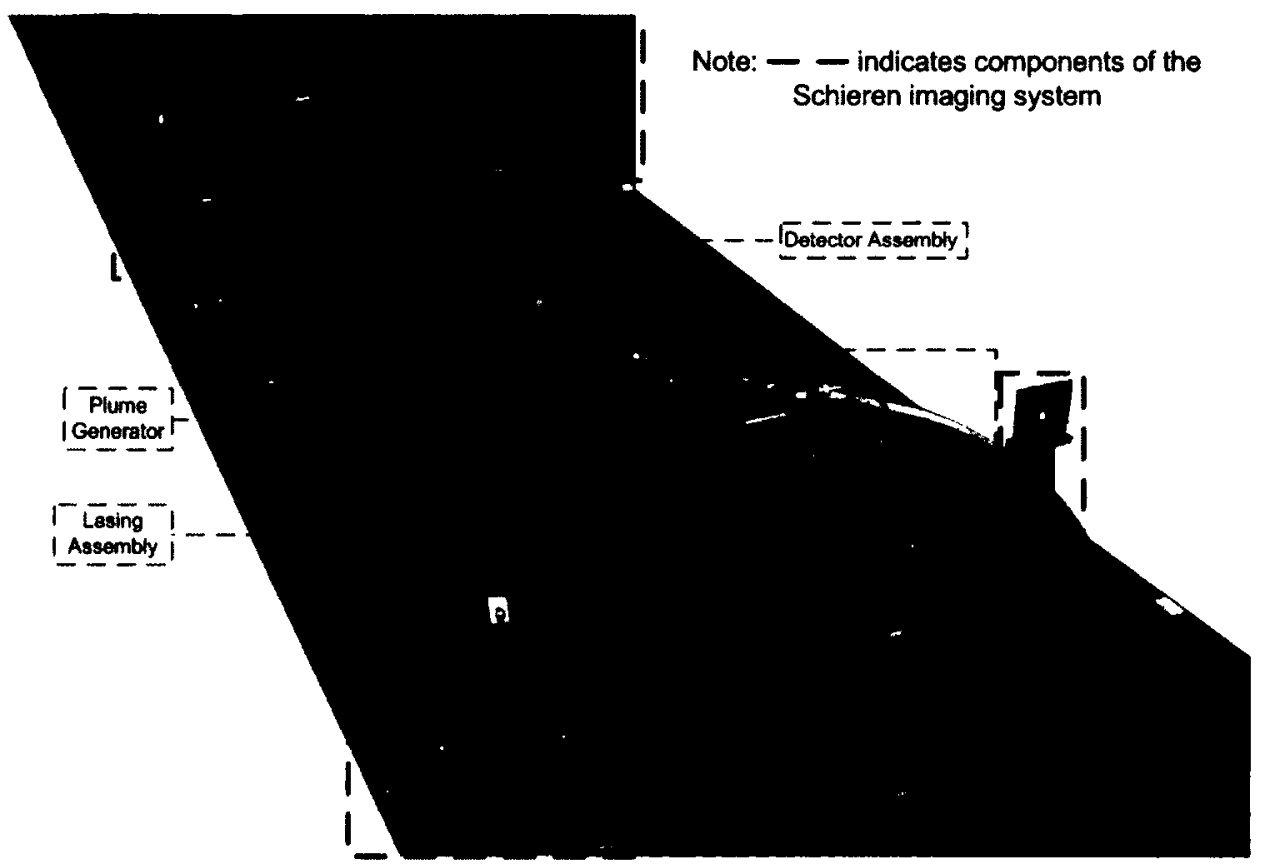

Figure 4.3: Experimental apparatus (with plume generator) 


\subsubsection{Lasing Assembly}

As shown in Figure 4.4, a HeNe laser (REO-2911-355) was mounted onto a CNC machined aluminum block via a V-mount base (Edmund Optics; NT03-665). The $v$-mount allowed precise centering of the laser and constrained the HeNe laser to two degrees of freedom relative to the spacer block (i.e. rotation and translation along the laser emission axis). The aluminum spacer was machined to ensure parallel mounting surfaces ( \pm 0.001 "), thereby limiting the introduction of additional alignment error. The $\mathrm{HeNe} / \mathrm{v}$-mount/spacer block assembly was mounted onto the optical table via a Thorlabs linear translational stage (LT101/M); however, the translational stage was implemented primarily as a mounting medium between the optical table and the spacer block and therefore was held fixed at all times. Alignment control between the laser and detectors was constrained to the detector side (See Section 4.2.3 for further detail).

The laser emission optics assembly was aligned with the $\mathrm{HeNe}$ laser via a mounting adaptor shown in Figure 4.5. The mounting adaptor was CNC machined to provide precise alignment between the HeNe laser front face (4 eq. spc. Ø36 mm 4-40 UNC) and the $30 \mathrm{~mm}$ cage system. The $30 \mathrm{~mm}$ cage system supplied by Thorlabs was a modular system that allows accurate and efficient alignment between $30 \mathrm{~mm}$ cage components (i.e. filter holder, optic cubes etc.). The lasing optics comprised a neutral density filter mounted within a quick release 1" filter holder, two pellicle beam splitters, a gold turning mirror, a $30 \mathrm{~mm}$ cage $\mathrm{V}$-mount, a $\mathrm{CaFl}_{2}$ lens mounted within a cage mount 1" filter holder, and a PVI-3.4 Mid-IR detector. 


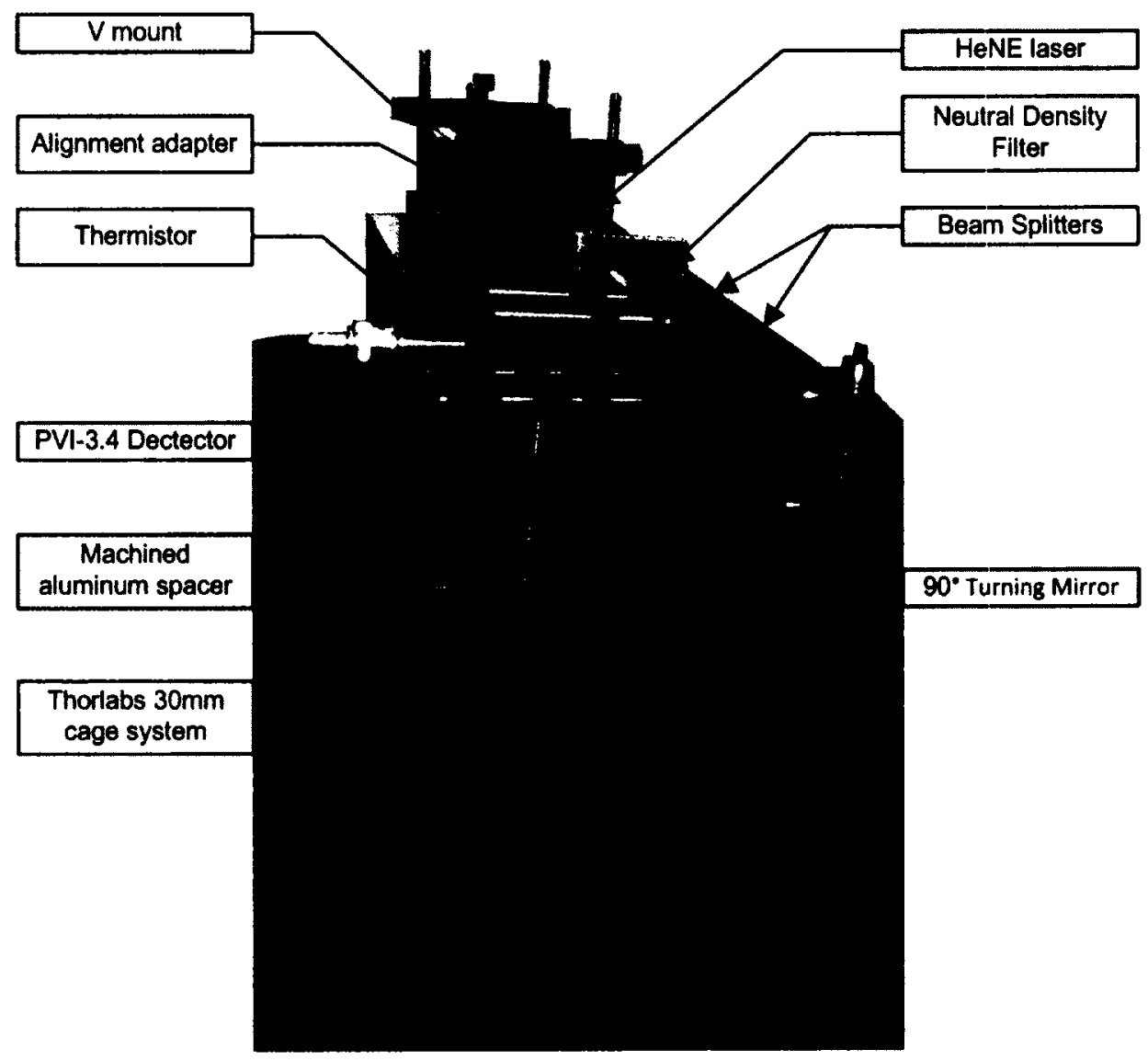

Figure 4.4: Lasing assembly of the VOC measurement sensor

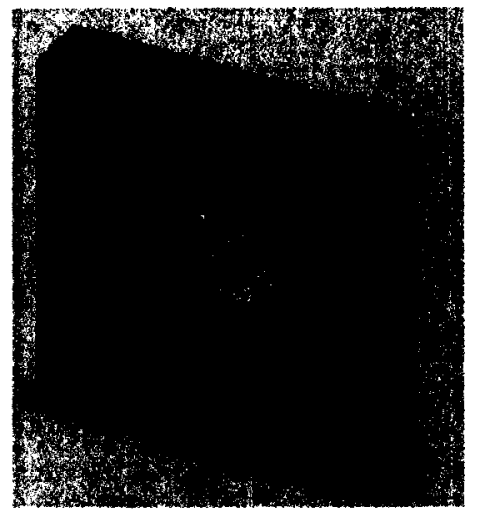

Figure 4.5: HeNe Laser $-30 \mathrm{~mm}$ cage alignment adapter

The 0.3 Optical Density (OD) neutral density filter (Edmund optics; NT64-353) is mounted onto the $30 \mathrm{~mm}$ cage system (Thorlabs) between the He-Ne laser and the first pellicle beam splitter via a quick release 1" optic holder (Thorlabs; QRC1A). The filter 
has a flat transmission ( $46 \%)$ curve across the experimental spectral bandwidth, as shown in Figure 4.6. This ensures that all three detectors (Vigo System; PVI-3.4) operate below their saturation limits (of $1 \mathrm{~mW} / \mathrm{mm}^{2}$ ) even at the HeNe's max operating power of $5 \mathrm{~mW}$.

Pellicle beam splitters were used to split the incoming laser intensity among the reference detector and the two detection lines. They are documented by Thorlabs (2010) and mentioned by Lyle (2005) to be the best choice when dispersion must be kept to a minimum. They virtually eliminate multiple reflections that are commonly seen with the use of thicker glass beam splitters known as ghosting. This phenomenon develops due to the secondary reflection within the optic and causes increased optical power losses and a secondary beam that is displaced from the original beam by the thickness of the optic. However, they are immensely fragile and can be easily destroyed or damaged through any surface contact. Through the use of Thorlabs beam splitter $30 \mathrm{~mm}$ cage cubes, the pellicle beam splitter can be protected and implemented into the in-lab sensor design without risk of damage. The pellicle beam splitters are also a viable choice for the future field sensor so long as additional precautions are taken to ensure safety from the atmospheric particulates.

The first pellicle beam splitter (Thorlabs; CM1-BP145B4) directs a portion of the laser intensity onto the reference detector. As noted earlier, the Mid-IR detector, v-block mounting platform, $\mathrm{CaFl}_{2}$ lens and pellicle beam splitter cube were held rigidly together using the $30 \mathrm{~mm}$ cage system, ensuring proper alignment the optical components along the laser propagation axis. To ensure proper focusing of the laser light onto the detector's active area, an $f=40 \mathrm{~mm} \mathrm{CaFl}{ }_{2}$ Plano-convex lens (Thorlabs; LA5370-E) with a specialized Anti-Reflective (AR) coating was utilized. The AR coating acts as a weak band pass filter across the (3-5 $\mu \mathrm{m})$ Mid-IR region. The detector V-mount platform (Thorlabs; ARV1) was used to align and centre the detector with the focal point of the $\mathrm{CaFl}_{2}$ lens within the $30 \mathrm{~mm}$ cage, preventing power losses through misalignment. The spectral response curves for the $\mathrm{AR}$ coated $\mathrm{CaFl}_{2}$ lens and the PVI-3.4 ( $\left.\mathrm{HgCdTe}\right)$ detector can be seen in Figure 4.7. 

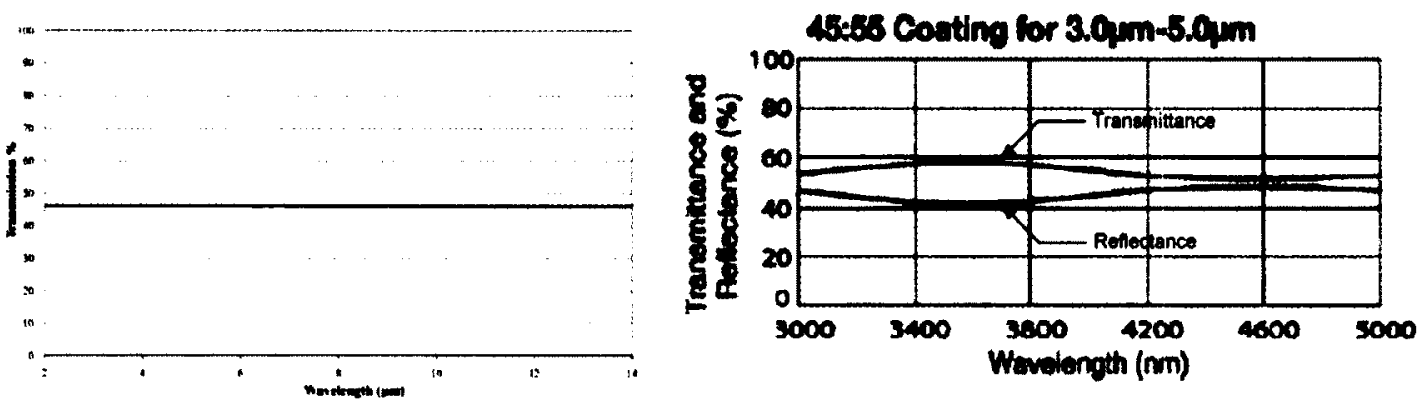

Figure 4.6: Spectral transmission curve of (Left) IR Neutral Density Filter $(O D=0.3)$; (Right) Specialized pellicle beam splitter
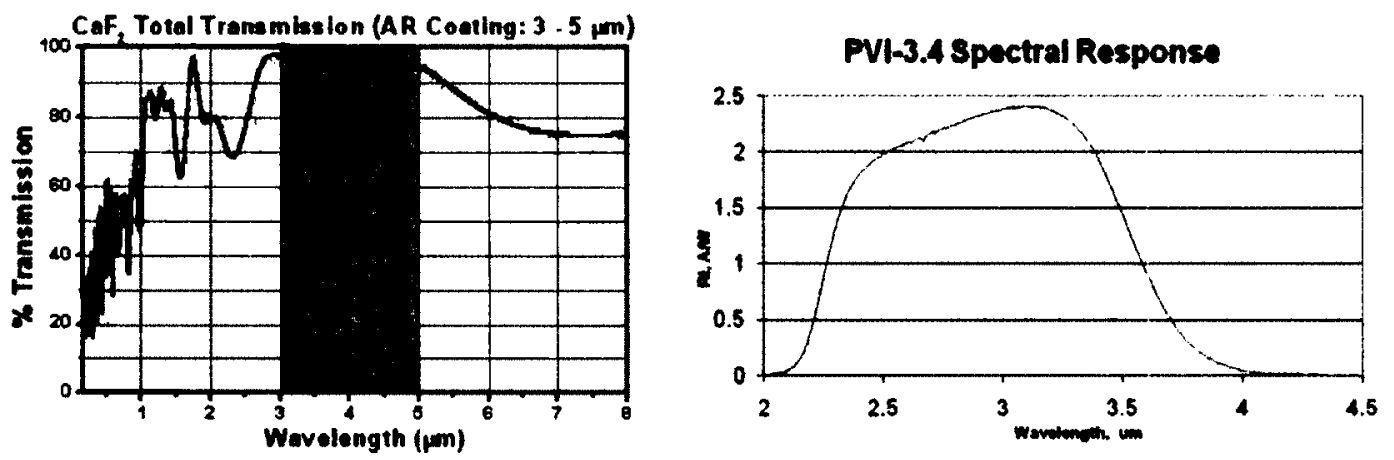

Figure 4.7: (Left) Spectral transmission of $\mathrm{f}=40 \mathrm{~mm} \mathrm{CaFl}_{2} \mathrm{Plano}$-convex lens; (Right) Spectral responsivity of the PVI-3.4 detector.

The final section of the lasing assembly comprised of a second pellicle beam splitter (Thorlabs; CM1-BP145B4) and a gold plated mirror (Thorlabs; CM1-M01) were utilized to create the two laser interrogation lines required for the $\mathrm{CCV}$ technique. See Figure 4.8 for the spectral response curve of the $90^{\circ}$ gold turning mirror. Due to the fact that $30 \mathrm{~mm}$ cage cubes were used to house the fragile optics, their physical makeup also determined the minimum achievable beam separation distance of $39.1 \mathrm{~mm}$. The distance between the measurements (i.e. laser beam separation) is a critical parameter that requires optimization (Rockwell, Rangwala, \& Klein, 2009), due to the flow's turbulent behaviour. Essentially, if there is high eddy dissipation, the magnitude of the fluctuation being measured by the first sensor will have no secondary fluctuation in the second sensor reading, precluding reliable cross-correlation. However, the current setup did not allow for the separation distance to be easily varied and it remained fixed at $39.1 \mathrm{~mm}$ for all experiments. The implications of this are discussed in the results and discussion. 


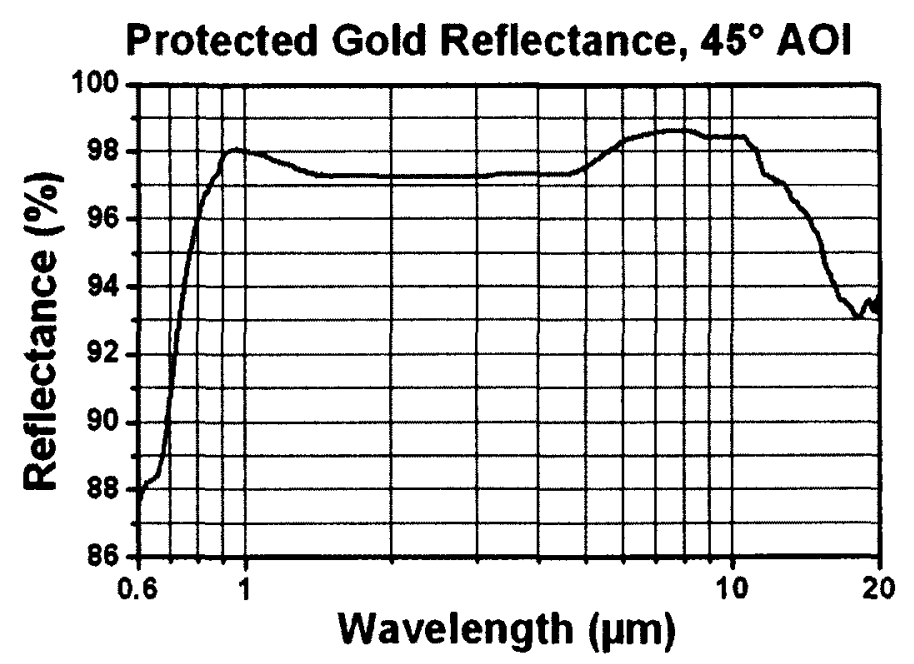

Figure 4.8: Spectral Reflectance curve for the gold plated mirror (Thorlabs Ltd., 2011)

The detectors specified for the experiment operate at room temperature (i.e. no temperature control) and have responsivity that fluctuates as a function of temperature. Therefore, thermistors (Omega; ON-930-44004) were mounted onto the detector's aluminum body using thermo-conductive adhesive, to monitor the operating temperature of the detector during experiments. The thermistors were connected via a simple voltage divider circuit to create useable voltage readings. The schematic of the thermistor circuit and its response/calibration curve is provided in Figure 4.9. The true zero positions of the thermistors were calibrated using an ice-water bath within a thermally insulated container. The deviations were found to be within the maximum range of $\pm 0.2^{\circ} \mathrm{C}$ from the true zero, which is the reported uncertainty of the sensor. For cross-correlation velocimentry tests and concentration tests, the thermistors voltage were sampled at frequencies of $15 \mathrm{~Hz}$ and $1000 \mathrm{~Hz}$ respectively, at 16 bit resolution using a National Instruments data acquisition card (NI PCI-6259). The thermistors monitored the operating temperatures of the PVI-3.4 detectors to ensure that the detectors responsivities were steady during experimentation. During the experiments, the detector temperatures remained within $\pm 0.3^{\circ}$ Celsius. 

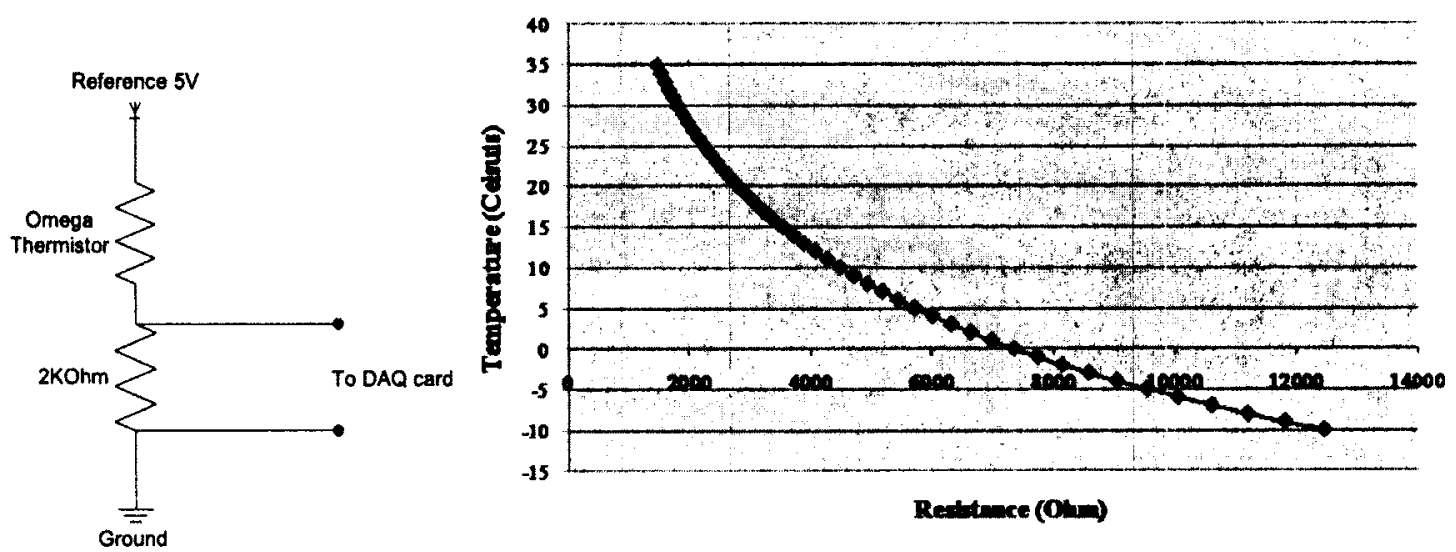

Figure 4.9: (Left) Thermistor's electrical circuit; (Right) Calibrated temperature response curve of the Omega thermistor

\subsubsection{Test Gas Delivery System}

The schematic of the test gas delivery system can be seen in the figure below.

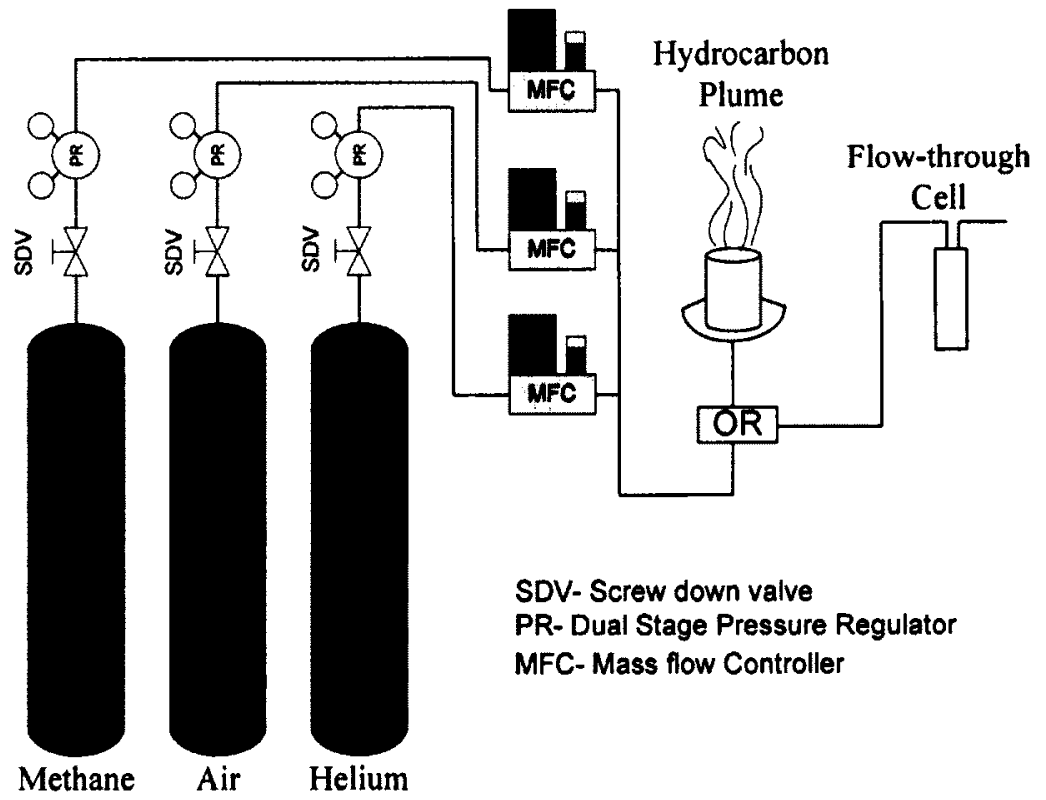

Figure 4.10: Schematic test gas delivery system and its components

The experimental studies were completed using two sets of gas mixtures, methane in air and methane in helium. The motivation of the second gas mixture was to enhance the contrast of the flow visualization created by the Schlieren imaging system discussed in Section 4.4, at higher flow rates. The delivery system includes Brooks mass flow controllers (Smart Link Advantage (SLA) models 5850S (2), 5851S (1)) that regulate the flow of each gas component from their respective pressurized cylinders. Experiments 
were conducted at total flow rates ranging from 0.3 to 10 Standard Litres per Minute (SLPM; where standard is defined at a temperature of $21^{\circ} \mathrm{Celsius}$ and $101,325 \mathrm{~Pa}$ ). For the $50.4 \mathrm{~mm}$ diameter plume generator, these flow rates translated to average velocities of 0.02 to $0.84 \mathrm{~m} / \mathrm{s}$.

The MFC's were calibrated one of two ways, using a bubble flow calibrator (Gilibrator, model 800271) for the small flow controllers (SLA 5850S), or using mass displacement for the larger flow controller (SLA 5851S). The bubble flow calibrator operates by measuring the time required for a bubble to pass between two infrared sensors in a vertical tube. Since the volume between the two infrared sensors is known, the volumetric flow rate can be determined from the extracted time delay. The mass displacement method employs a high-precision weight scale to measure the mass depletion rate of the pressurized ('Q'-size) bottle of the calibration gas, by which the volumetric flow rate can be determine for the given ambient conditions. Both methods determine the steady state volumetric flow rate for specific set point voltages $(0.25,0.5$, $0.75,1.0,1.75,2.5,3.75,4.25,4.75$, and 4.95 volts; max being 5 volts) from which calibration curves are generated for the operating ranges (SLPM vs. set point voltage) of each MFC, as seen in Figure 4.11. The calibrated MFCs were found to perform with an uncertainty of \pm 1.2 percent of the set flow rate.

\section{Brooks (Methane)SLA5050S}

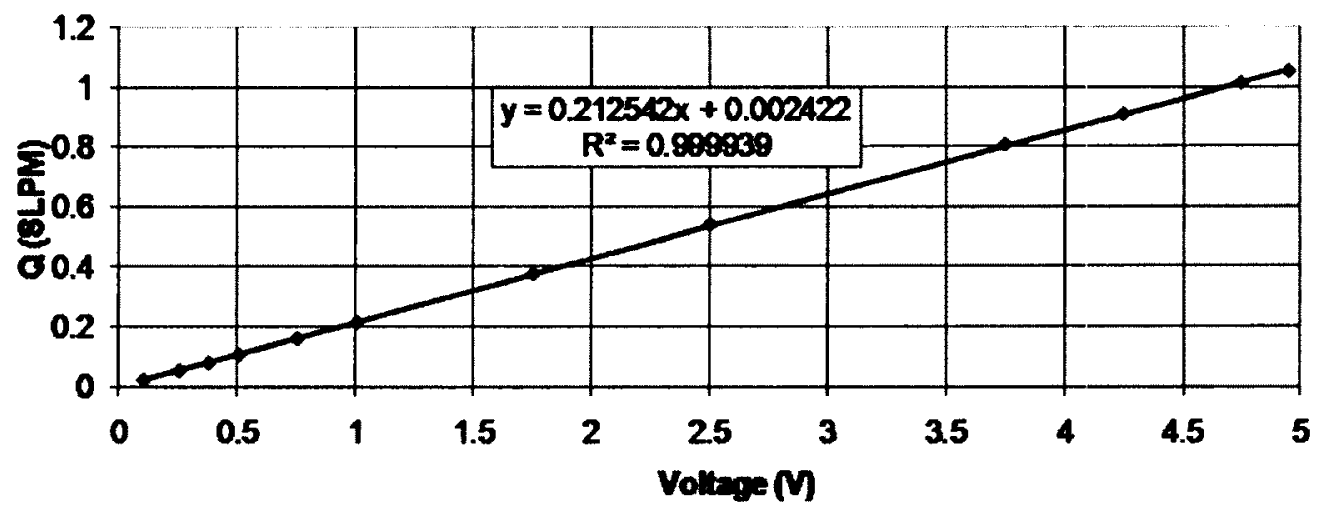

Figure 4.11: Example of a calibration curve for Brooks MFC 


\subsubsection{Plume generator}

The plume generator, seen in Figure 4.12, is designed to act as settling chamber for the incoming gas jets while promoting quasi-uniform, vertical flow through the use of a perforated metal outlet.
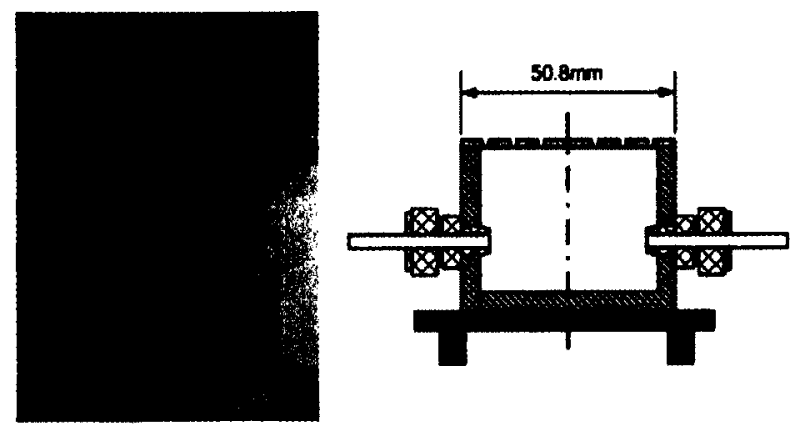

Figure 4.12: Plume generator for CCV spectroscopic study.

\subsubsection{Spectroscopic flow-through cell}

A flow-through cell (Mandel Scientific; SN-71B-Q-10, see Figure 4.13) was used to determine the VOC sensor concentration sensitivity. The cell had Quartz windows that allowed $\sim 70 \%$ transmission across the desired spectral bandwidth $(3.392 \mu \mathrm{m})$. The reported tolerance on the $10 \mathrm{~mm}$ path length through the cell volume $(3.0 \mathrm{~mL})$ is $\pm 0.01 \mathrm{~mm}$, resulting in an uncertainty contribution of $0.1 \%$ in path length.
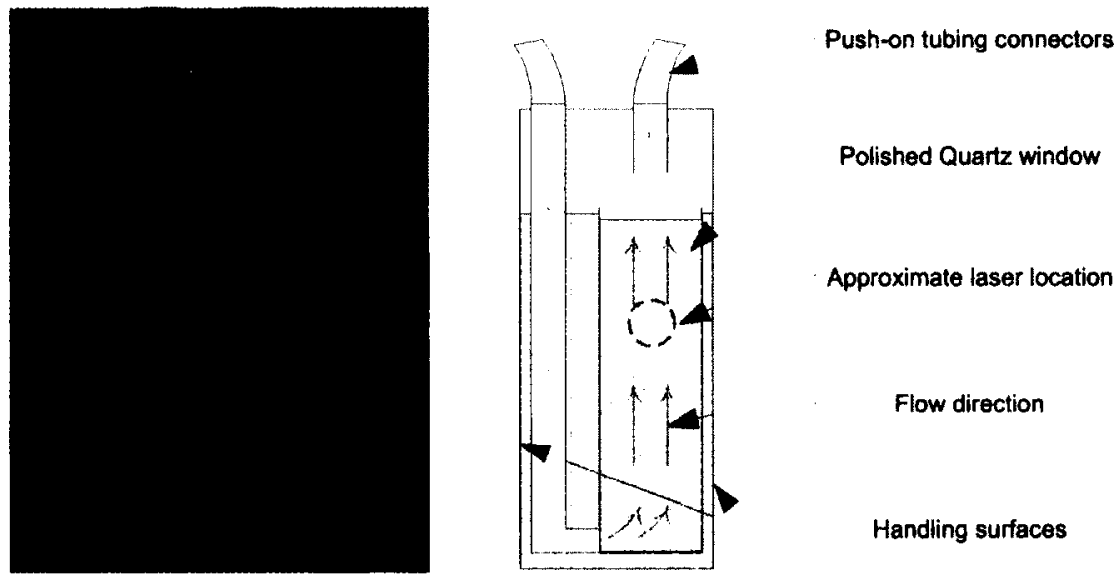

Figure 4.13: Spectroscopic flow-through cell 


\subsubsection{Detector Assembly}

As shown in Figure 4.14, the detector assembly at the end of the two laser interrogation lines was designed similarly to the reference detector section, except that each detector has its own means of translation to aid alignment. There were two means of $x$-direction translational control for each detector assembly: 'coarse' alignment was achieved through the translation of the $30 \mathrm{~mm}$ cage within the cage holder attached to the post, while 'fine' control was achieved using a translation stage beneath the cage assembly (Newport; M433). A vertical translation post (Edmund optics; NT84-350) allowed for precise (1 mm travel for $45^{\circ}$ rotation; $10 \mathrm{~mm}$ total travel length) alignment of each detector cage. The segmented nature of each detector assembly provided a greater degree of freedom, enabling compensation for any errors in alignment in the lasing section. Due to the physical spacing restrictions caused by the V-block (Thorlabs; ARV1) detector mounting platform, gold plated turning mirrors (Thorlabs; CM1-M01) were utilized to divert the laser beams by $45^{\circ}$ in opposite directions. Each laser beam was then focussed onto a PVI ( $\mathrm{HgCdTe}$ ) detector using a specially coated $f=40 \mathrm{~mm} \mathrm{CaFl} \mathrm{plano}$-convex lens. Since beam steering caused by the hydrocarbon plume was found to be insignificant, detailed in Appendix D; thus larger focusing optics for the full-scale sensor will not be required. An Omega thermistor was mounted onto each detector to monitor the operating temperature.

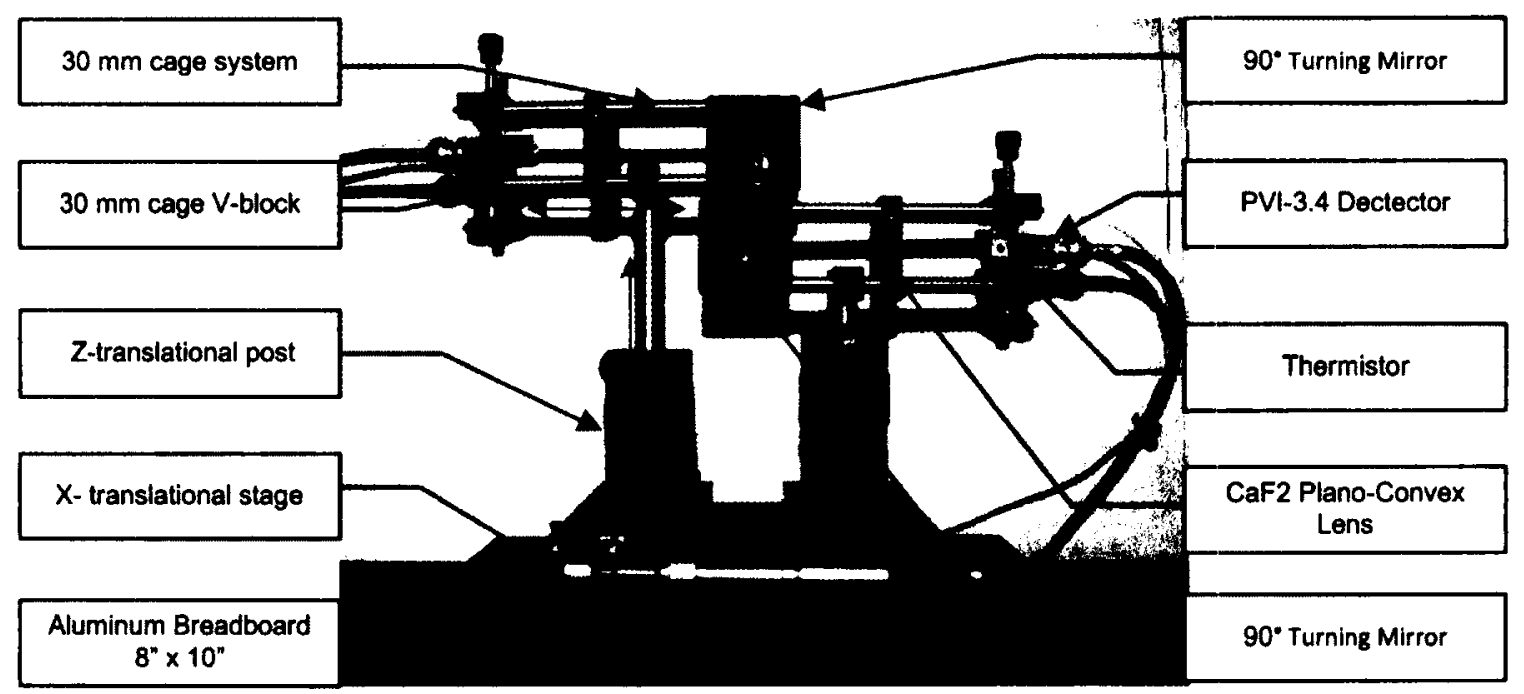

Figure 4.14: Detector Assembly of the VOC measurement Sensor 


\subsection{Optical Power Conversion Electronics}

Since the HgCdTe PV detector generates current in the $\mu \mathrm{A}$ range proportional to the incident power, direct monitoring would require an expensive current acquisition card. A common solution to this dilemma is to use a transimpedance amplifier to convert the smaller current signal into a more readily usable voltage signal. To minimize the costs and to provide a greater degree of control, custom detector amplifiers were designed and constructed. The custom amplifier was built at $1 / 6^{\text {th }}$ the commercial cost of standard amplifier, while still providing the desired 3-stage control: raw signal conversion/amplification, DC subtraction, and AC (only) amplification.

To design a transimpedance stage that did not degrade the raw detector signal with ' $1 / \mathrm{f}$ ' noise (common to most OPAMPs), specialized OPAMPS (OPA380A) were utilized. The OP380A noise frequency distribution was found to have a flat spectral response of $110 \mathrm{nV} / \mathrm{J}(\mathrm{Hz})$ for the frequency bandwidth of interest (DC-1 KHz). However, the voltage output range of the OPA380 is relatively small $(0.02-4.5 \mathrm{~V})$. To prevent amplifier saturation, it was determined that a variable gain stage should be incorporated. The design of stage 1 (raw signal conversion/ amplification) is shown in Figure 4.15 below. The gain resistors were selected based on the current $\mathrm{min} / \mathrm{max}$ output range of the detector and the output voltage capacity of the OPA380A operational amplifier.

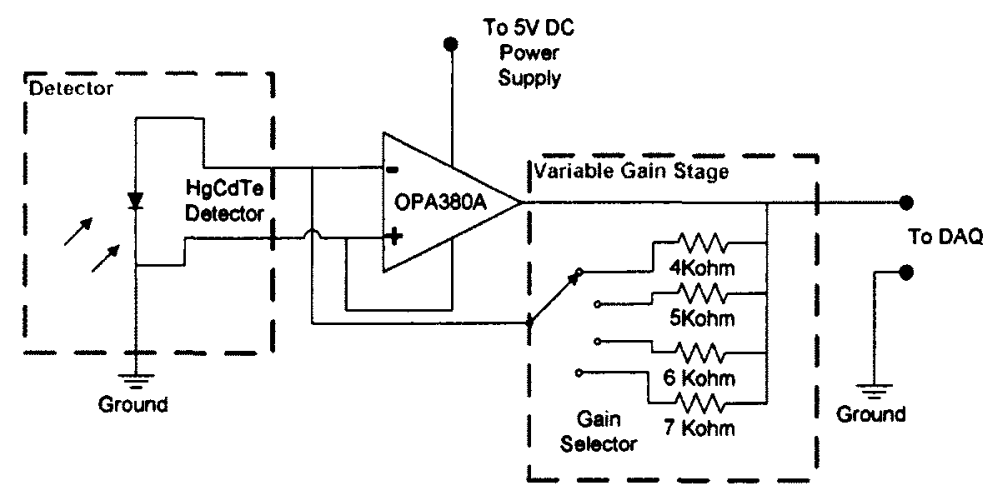

Figure 4.15: Stage 1- Transimpedance amplifier with variable gain

For measurements in buoyant plumes, two main parts of the "plume absorption" signal were expected: a DC signal corresponding to the mean absorption and an AC 
signal that is created from the fluctuations (i.e. eddies) in the flow. To effectively capture the velocity of the flow from low concentration measurements, it was determined that the cross correlation algorithms would provide better results if implemented on the $\mathrm{AC}$ component of the signal for low concentration measurements (i.e. the DC \& AC signal can be used to measure the path integrated concentration across the plume-laser line and the AC component can be utilized to measure the velocity of the plume's transient fluctuations). However, to maintain good sampling resolution (from the DAQ) over the $\mathrm{AC}$ component of the signal, it was deemed that an analog $\mathrm{AC}$ extraction and amplification circuit should be constructed to allow the user to manually set the DC offset. The stage 2 gain was set to two (2) because it is the lowest possible achievable gain in the non-inverting operational amplifier circuit due to the circuit configuration. Since the main function of this stage was AC extraction and not amplification, the lowest possible gain was selected to prevent OPAMP saturation. The amplification was segmented into a separate stage to allow precise control of the AC signal and to prevent amplifier/DAQ voltage saturation. A schematic of stage 2 is shown in Figure 4.16.

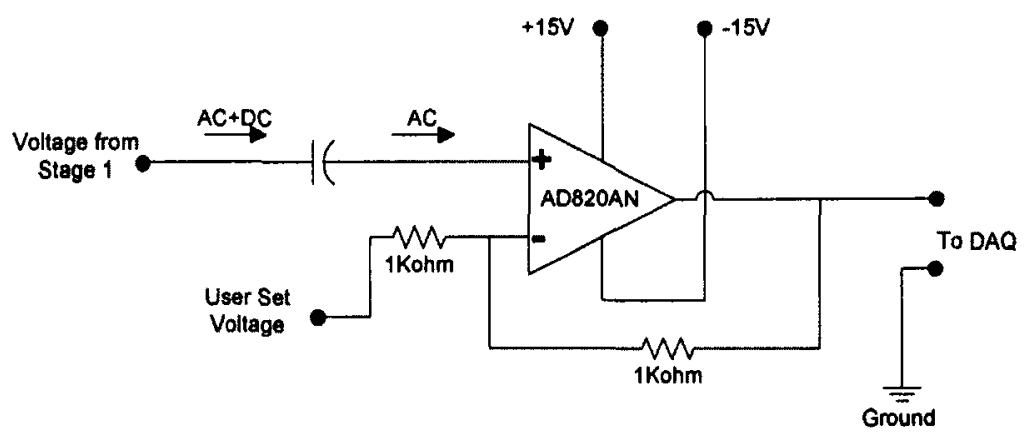

Figure 4.16: Stage 2-AC extraction circuit with fixed (X2) gain

The final AC amplification stage is shown in Figure 4.17, where the range of AC amplification (i.e. amplification) was selected based on the minimum expected fluctuation intensity of $(0.02 \%)$.The amplifier design was finalized through testing and verification in National instruments (NI) Multisim 11.0, using a simulated signal of 10 percent fluctuations in the detector signal. It was found that the operational amplifiers provided the desired conversion/ amplification while maintaining good noise characteristics. 


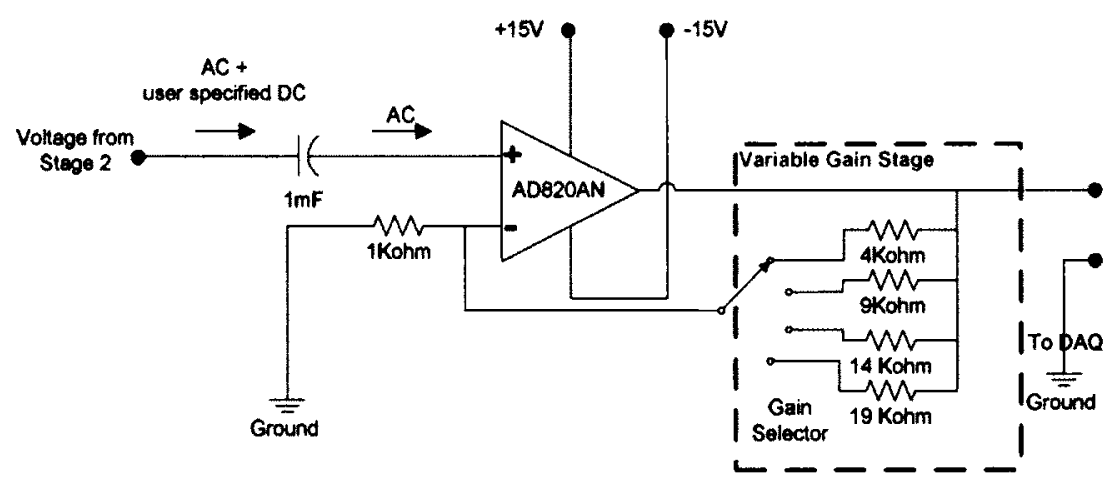

Figure 4.17: Stage 3 - AC amplification with variable gain.

To provide added shielding to the amplifier circuits, the final circuit was constructed on a Printed Circuit Board (PCB) and mounted within a metal enclosure, as shown in Figure 4.18. The PCB design was completed using EAGLE ver. 5.6 and printed by BatchPCB located in Boulder, CO. The completed PCB design can be seen in Appendix B

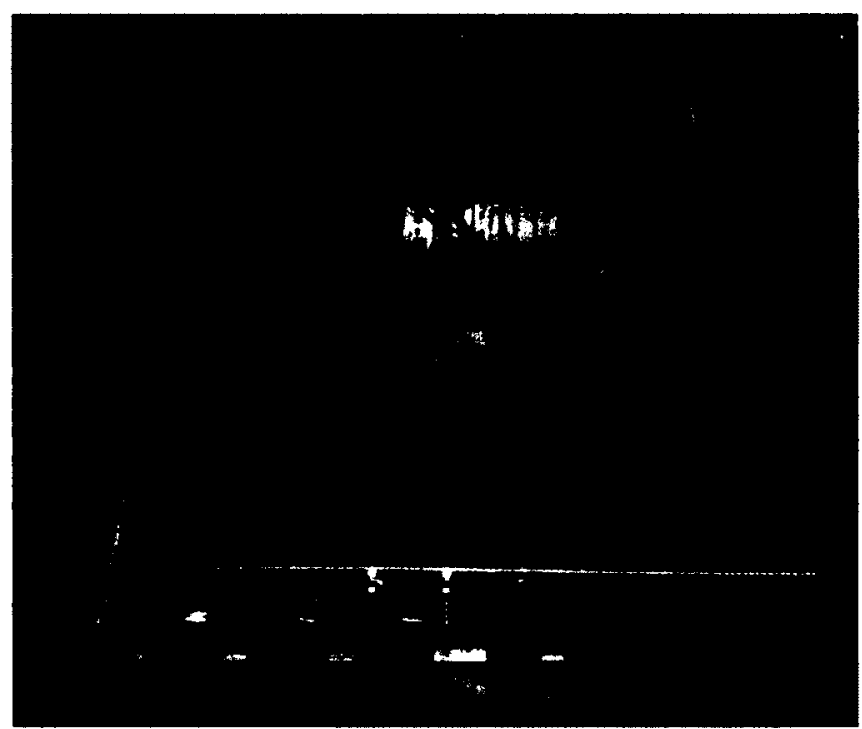

Figure 4.18: Custom "in-house built" detector amplifier

\subsection{Flow Visualization Apparatus}

To qualitatively estimate/corroborate the velocity extracted from the cross correlation technique and to elucidate the perceived trends of the line flux measurements, a Schlieren imaging system was constructed to create $2 \mathrm{D}$ visualizations of the generated plume. The 
Schlieren imaging technique utilizes the fundamental linear relationship between the refractive index and the gas density (e.g. Settles (2001), to produce contrast images that are proportional to the spatial density gradients. The methodology requires parallel light rays to pass through the 'test section' (of different gas density than air) which in turn causes some light rays to bend away from their original path. As seen in Figure 4.19, the light passes through a (non-air) gas pocket which causes it to refract onto a new path ' $B$ '. Then by focusing the light onto an opaque filter (more commonly known as a knife-edge) that lies just outside the focal point, an image with a dark spot corresponding to the 'blocked' light can be generated. However, a Schlieren object refracts many such rays in many directions, the downward rays are blocked off and the top rays are not and propagate to the image plane. The overlay of the blocked light and the refracted light yields a gradient of the refraction that is also proportional to the gradient of density (Atcheson, 2007; Settles, 2001). The percent of light cut-off by the knife yields Schlieren images with proportional contrast and by that effect allows discerning of smaller density gradients. By using a vertical knife edge, a horizontal density gradient image can be generated because all the light refracted onto new angles on the horizontal plane will be blocked by the knife edge and vice-versa.

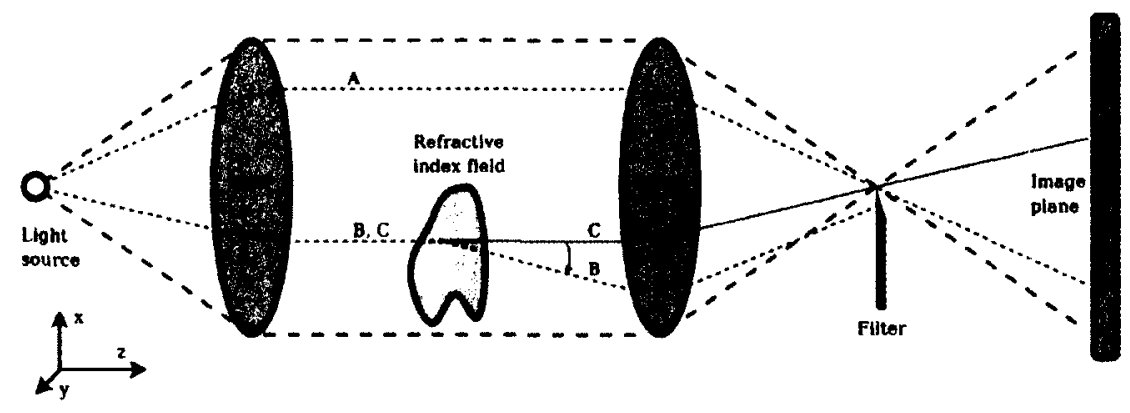

Figure 4.19: A simple lens-based Schlieren setup (Atcheson, 2007) 


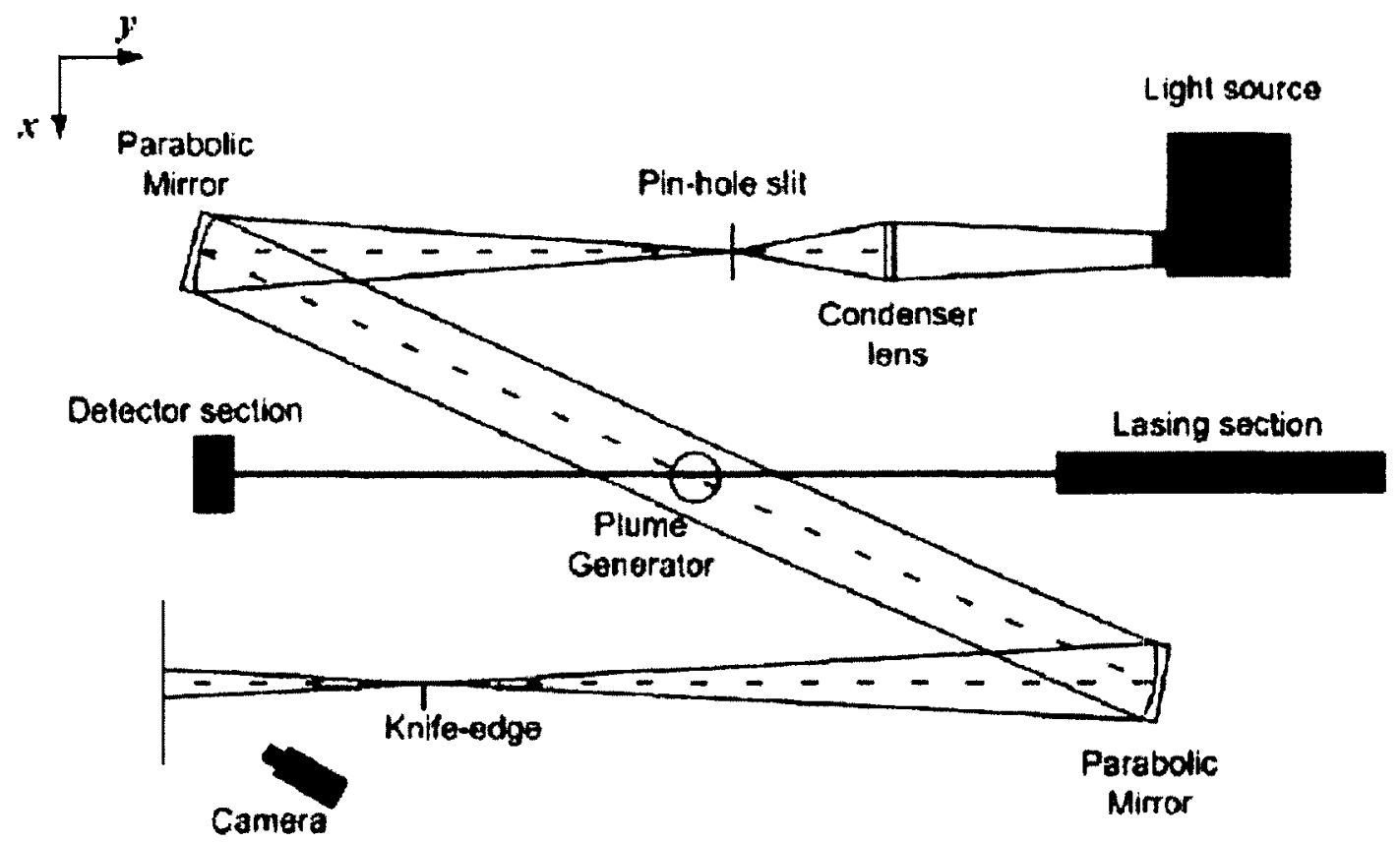

Figure 4.20: Z-type Schlieren imaging system (Top-view)

The Schlieren imaging system employed for the in-lab experimental was arranged in the common Z-configuration, as seen in Figure 4.20. A 2500 Lumens light source (Dell; 1900X), a $f=500 \mathrm{~mm}$ double convex 'condenser' lens and a 'pin-hole slit' filter were utilized to create a uniform point light source, required to create parallel light rays that transverse the plume generator. The light source and the condenser lens were mounted onto 2 tripods that allowed proper alignment through their respective 4 stage control (z-axis translation and pitch, roll and yaw). The pin-hole filter was mounted onto the optical granite table via an $x$-translational stage (Edmund optics; NT36-447) and a $z$ axis translation post (Edmund optics; NT84-350) to provide alignment control in the $x-z$ plane (perpendicular to the light path). Using the translation stages, the pinhole was traversed through the focal point of the collimated light until an optimal position was ascertained, which provided the most uniform light source (as determined by visually inspecting the "pre-cut-off" light projected onto the light screen). The two parabolic mirrors $(f=1524 \mathrm{~mm}$, Edmund optics; NT32-084) were mounted onto the granite table using dovetail translation stages (Thorlabs; DT25) and rotation platforms (Thorlabs; 
RP01) to provide coarse positional control. Finer mirror adjustments were achieved through the $3^{\circ}$ tilt-yaw control of the parabolic mirror mount (refer to Figure 4.21).

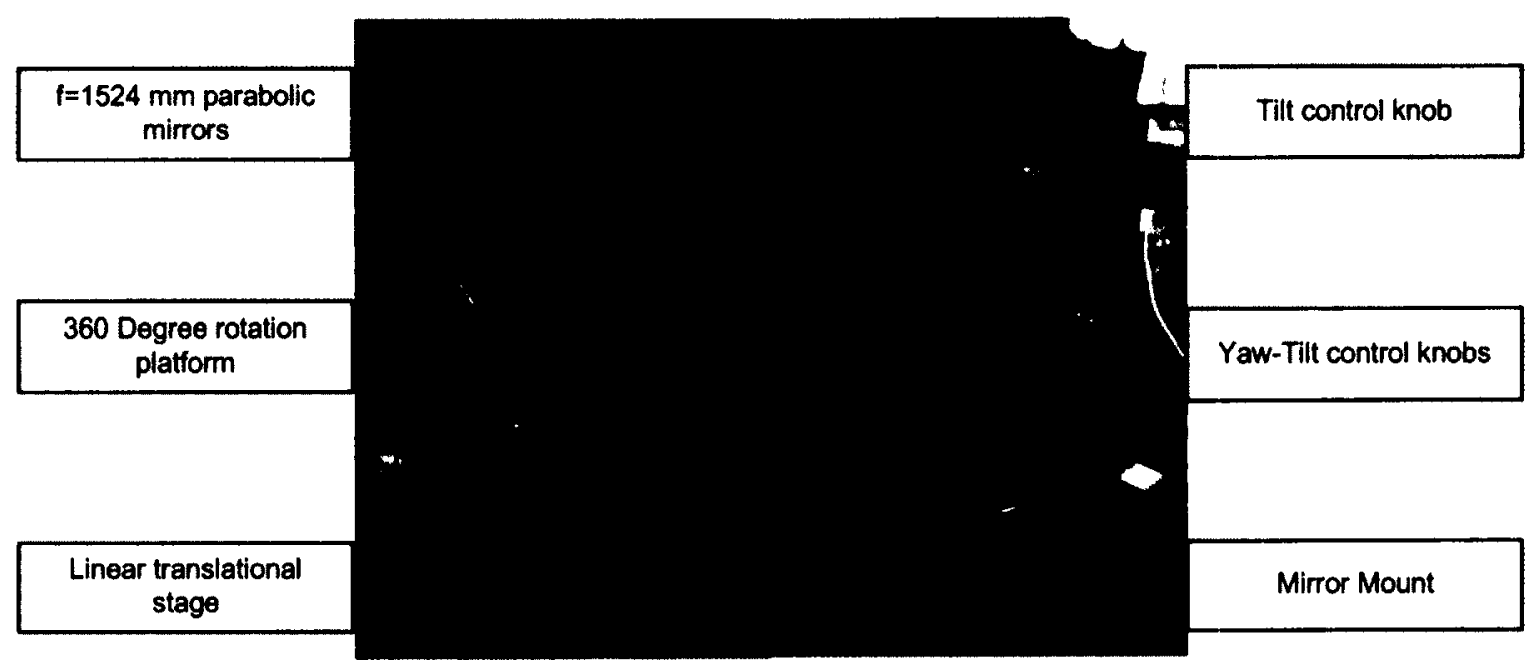

Figure 4.21: Parabolic mirrors used for Schlieren imaging system

After the light is reflected off the $2^{\text {nd }}$ parabolic mirror, the light beam is collimated at the focal length of the mirror. The knife-edge is used to incrementally segment the light at the focal point until a proper balance between contrast and luminance is achieved. To capture images at a reasonable frame rate ( $>10 \mathrm{fps})$, the maximum light cut-off was kept below $50 \%$. The knife-edge (see Figure 4.22) was mounted onto the optical table via an $\mathrm{x}$-axis translation stage (Newport; M-26) and an aluminum spacer block. The camera utilized to capture the Schlieren images was an IEEE 1394a machine vision camera (Pixelink; PL-B762F) with an $8.5 \mathrm{~mm}$ fixed focal length lens (Edmund optics; NT58-000). The camera was controlled via Pixelink capture OEM, a user friendly Graphical user interface (GUI) that allows direct control of all the desired settings (exposure time, fps, gain etc.). 


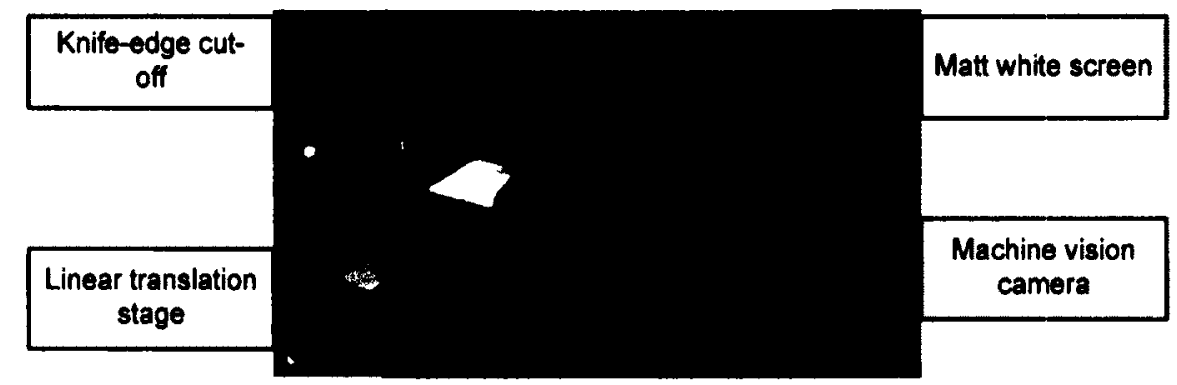

Figure 4.22: (Left) Knife-edge assembly; (Right) Schlieren Image and acquisition camera

\subsection{Experimental Methodology}

\subsubsection{Line-Averaged Concentration Measurements}

Figure 4.23 shows a schematic of the methodology used to determine the volumetric fraction of methane within the plume. The detector signal gains specified via the custom amplifier were kept at the minimum setting to gauge the worst case scenario SNRs and sensitivities. The analog detectors and thermistor were then sampled at $1000 \mathrm{~Hz}$ and 16 bits, and the pressure transducer was sampled at $1 \mathrm{~Hz}$ at 14 bits via Omega's built in Ethernet API.

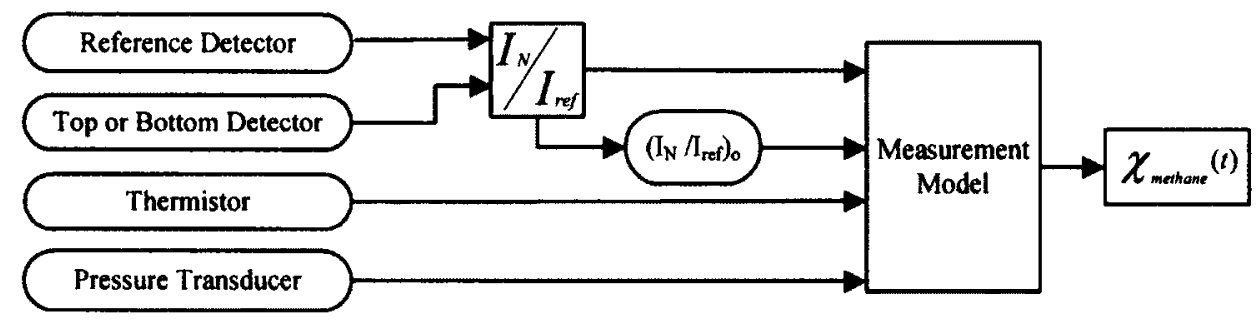

Figure 4.23: Mid-IR DAS methodology

As mentioned earlier, to benchmark the new cost-effective Mid-IR VOC sensor's concentration sensitivity, a flow through cell was utilized to conduct controlled (fixed path-length) tests with different mixtures of methane in air. The sensitivity tests were conducted on a range of $0-10 \%$ methane in air mixtures, which was equivalent to $0-$ $1000 \mathrm{ppm}-\mathrm{m}$ when considering the $10 \mathrm{~mm}$ path-length of the spectroscopic flow through cell. Following laser noise rejection, the volumetric fraction of methane was determined via the Beer-Lambert law. The laser noise rejection was achieved by normalizing the 
'absorption' signal with the reference detector signal. From the normalized detector readings, the base power (i.e. with no absorption) was recorded to calculate the fractional transmission of laser light $\tau_{R}$, via Eq. 4.1.

$$
\tau_{R}=\frac{I_{N} / I_{\text {ref }}}{I_{N} /\left.I_{\text {ref }}\right|_{o}}
$$

where:

$I_{N} / I_{\text {ref }}$ is the normalized detector signal [-], and

$I_{N} /\left.I_{r e f}\right|_{O}$ is the normalized detector signal with no absorption[-].

Next, the absorption coefficient was determined via Eq. 4.2 considering the gas temperature(measured with an Omega ON-930-44004 thermistor) and barometric pressure (measured with an Omega; iBTX-SD pressure transducer). The absorption coefficient was determined before every test run to limit the error introduced by its temperature/pressure dependencies. The calibration tests were conducted with $10 \%$ methane in air, at $90 \%$ of the MFC's full range to limit any added uncertainty in the measurements from the MFC. From the set volumetric fraction of methane, as well as the measured transmission of laser light $\tau_{R}$, temperature and barometric pressure, the absorption coefficient could be determined via Eq. 4.2,

$$
\sigma_{C H_{4}}=\frac{-R \cdot T}{P_{A^{\prime}} \cdot \chi_{C H_{4}}} \ln \left(\tau_{R}\right)
$$

where:

$\sigma_{\mathrm{CH}_{4}}$ is the molar absorption coefficient of the methane $\left[\mathrm{m}^{2} / \mathrm{mol}\right]$,

$\chi_{\mathrm{CH}_{4}}$ is the set volume fraction of methane in air[-],

$R$ is the universal gas constant [ $\mathrm{J} / \mathrm{K}-\mathrm{mol}]$,

$P$ is the barometric pressure $[\mathrm{Pa}]$,

$T$ is the temperature of the gas mixture $[\mathrm{K}]$,

$L$ is the traverse laser path length through the gas mixture [m], and

$\tau_{R}$ is the fractional transmission of the laser light [-]. 


\subsubsection{Uncertainty analysis of $\sigma_{\mathrm{CH}_{4}}$ and $\chi_{\mathrm{CH}_{4}}$}

The total uncertainties of the measured absorption coefficient $\sigma_{\mathrm{CH}_{4}}$ and volumetric fraction $\chi_{\mathrm{CH}_{4}}$ were calculated in accordance with the ANSI/ASME Measurement uncertainty standard [1985]. The standard utilizes the Taylor Series Method (TSM) method to combine the systematic and precision errors of each measured variable to estimate a final total uncertainty. The systematic error (more commonly known as the bias error) is a measure of an instrument's inability to measure the true value. Precision error on the other hand is the random error or scatter of a repeated measurement. It should be noted that this approach is only directly applicable to measurements with Gaussian error distributions. The calculation of total uncertainty using the TSM method of combining component uncertainties can be seen in Eq. 4.3. (Coleman, 2009)

$$
\begin{gathered}
U_{r}^{2}=\left(\frac{\partial r}{\partial X_{1}}\right)^{2} U_{X_{1}}^{2}+\left(\frac{\partial r}{\partial X_{2}}\right)^{2} U_{X_{2}}^{2}+\cdots+\left(\frac{\partial r}{\partial X_{N}}\right)^{2} U_{X_{N}}^{2}=\sum_{i=1}^{N}\left(\frac{\partial r}{\partial X_{i}}\right)^{2} U_{X_{i}}^{2} \\
U_{X_{i}}^{2}=B_{i}^{2}+P_{i}^{2}
\end{gathered}
$$

where:

$U_{X_{i}}$ is the uncertainty associated with variable $X_{i}$,

$r$ is the result,

$B_{i}$ is the systematic uncertainty, and

$P_{i}$ is the precision uncertainty.

The precision uncertainty is determined through the following relation seen below.

$$
P_{i}=t_{v, 95 \%} \frac{s}{\sqrt{N}}
$$

where:

$t_{v, 95 \%}$ is the $95 \%$ confidence $t$ value from the student's distribution, $S$ is the standard deviation of the variable, and

$N$ is the number of samples.

The partial derivatives used to calculate the total uncertainty of the absorption coefficient $\sigma_{\mathrm{CH}_{4}}$ are listed below. It should be noted that the pressure drop that occurs from the spectroscopic cell to the end of the vent pipe was found to be negligible 
considering the fact that the highest calculated pressure drop was below the reported error $(<200 \mathrm{~Pa})$ of the pressure transducer. The uncertainties for each dependent variable and the total uncertainty are listed Table 4.1.

$$
\begin{aligned}
& \frac{\partial \sigma_{C H_{4}}}{\partial T}=\frac{-R \cdot T}{P_{A} \cdot L \cdot \chi_{C H_{4}}} \ln \left(\tau_{R}\right) \\
& \frac{\partial \sigma_{C H_{4}}}{\partial P_{A}}=\frac{R}{P_{A} \cdot L \cdot \chi_{C H_{4}}} \ln \left(\tau_{R}\right) \\
& \frac{\partial \sigma_{C H_{4}}}{\partial L}=\frac{R}{P_{A} \cdot L^{2} \cdot \chi_{C H_{4}}} \ln \left(\tau_{R}\right) \\
& \frac{\partial \sigma_{C H_{4}}}{\partial \tau}=\frac{-R \cdot T}{P_{A} \cdot L \cdot \chi_{C H_{4}}} \cdot \frac{1}{\tau_{R}} \\
& \frac{\partial \sigma_{C H_{4}}}{\partial \chi_{C H_{4}}}=\frac{R \cdot T}{P_{A} \cdot L \cdot \chi_{C H_{4}}{ }^{2}} \ln \left(\tau_{R}\right) \\
& \sqrt{\left(\frac{\partial \sigma_{C H_{4}}}{\partial T}\right)^{2}\left(U_{T}\right)^{2}+\left(\frac{\partial \sigma_{C H_{4}}}{\partial P_{A}}\right)^{2}\left(U_{P_{A}}\right)^{2}+\left(\frac{\partial \sigma_{C H_{4}}}{\partial L}\right)^{2}\left(U_{L}\right)^{2}+\left(\frac{\partial \sigma_{C H_{4}}}{\partial \tau_{R}}\right)^{2}\left(U_{\tau_{R}}\right)^{2}+\left(\frac{\partial \sigma_{C H_{4}}}{\partial \chi_{C H_{4}}}\right)^{2}\left(U_{\chi_{C H_{4}}}\right)^{2}}
\end{aligned}
$$

The uncertainty of $\tau_{R}$, was calculated from the precision uncertainty as seen in Eq. 4.5, where the number of samples was 60,000 and a t-value of 1.98 was used (as a conservative estimate) for student's t-distribution. The uncertainty of set $\chi_{\mathrm{CH}_{4}}$ was calculated from the uncertainties of the MFCs. To convert the standard flow rate (which is calculated based on standard temperature of $294 \mathrm{~K}$ and pressure of $101325 \mathrm{~Pa}$ ) to the actual flow rate for the given ambient conditions, Eq. 4.12 was utilized. This also results in an increase in relative uncertainty on the set flow rates, determined by Eq. 4.13. After calculating the uncertainty of the set flow rates, the uncertainty of the set $\chi_{\mathrm{CH}_{4}}$ can be calculated from the same TSM method seen in Eq. 4.14

$$
\begin{gathered}
Q_{L P M}=\left(\frac{T_{1}}{T_{0}}\right)\left(\frac{P_{0}}{P_{1}}\right) * Q_{S L P M} \\
\frac{U_{Q_{L P M}}}{Q_{L P M}}=\sqrt{\left(\frac{U_{T_{1}}}{T_{1}}\right)^{2}+\left(\frac{U_{P_{1}}}{P_{1}}\right)^{2}+\left(\frac{U_{Q_{S L P M}}}{Q_{S L P M}}\right)^{2}}
\end{gathered}
$$


$U_{\chi_{C H_{4}}}=\sqrt{\left(\frac{Q_{a i r, L P M}}{\left(Q_{C H 4, L P M}+Q_{a i r, L P M}\right)^{2}}\right)^{2}\left(U_{Q_{C H 4, L P M}}\right)^{2}+\left(\frac{Q_{C H 4, L P M}}{\left(Q_{C H 4, L P M}+Q_{a i r, L P M}\right)^{2}}\right)^{2}\left(U_{Q_{a i r, L P M}}\right)^{2}}$

From these uncertainties, the total uncertainty of the absorption coefficient of $1.3 \%$ was calculated where $\sigma_{\mathrm{CH}_{4}}$ was determined through calibration to be 16.831 $\pm 0.223 \mathrm{~m}^{2} / \mathrm{mol}$. From the absorption coefficient calculated from HITRAN (2008), seen in Figure 4.24, the absorption coefficient determined through the in lab calibration shows good agreement with the theoretical values. The approximate location of the $\mathrm{HeNe}$ laser's FWHM can be seen highlighted in red dash lines. Since the exact centre wavelength and the beam profile were not available it was deemed that in-lab calibration was sufficient to determine the molar absorption coefficient of methane.

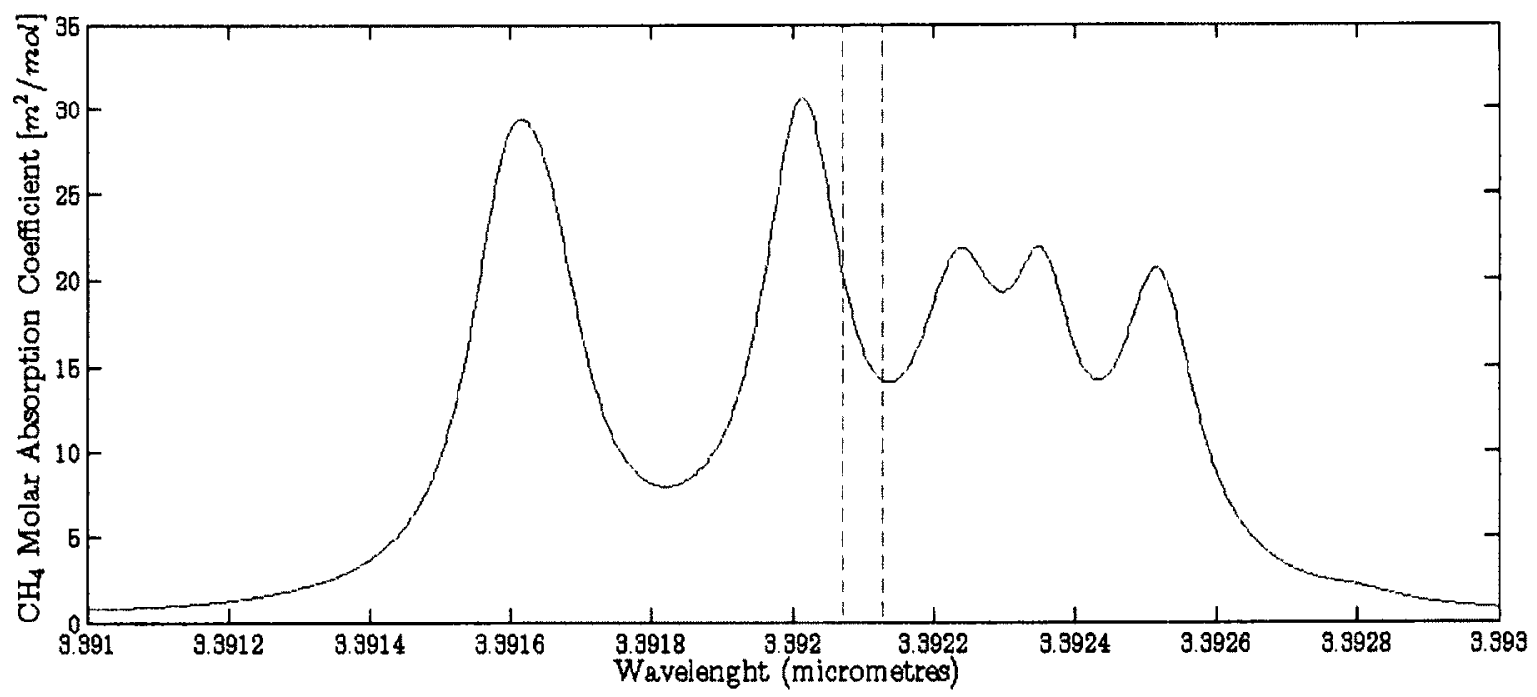

Figure 4.24: Molar Absorption coefficient of $\mathrm{CH}_{4}$ distribution across the mid-IR OSR; the approximate location of the HeNe laser beams FWHM is highlighted in dashed red lines (HITRAN, 2008)

From the uncertainty analysis, it can be seen that the majority of the uncertainty in the measured absorption coefficient comes from the uncertainty in the volumetric fraction of the gas mixture, seen in Table 4.1. It stands to reason that further improvement in the uncertainty can be achieved by using MFCs with higher accuracy. 
Table 4.1: Relative uncertainties of the measured/calculated parameters

\begin{tabular}{|c|c|}
\hline Constituent & Relative Uncertainty \\
\hline Temperature & $0.06 \%$ \\
\hline Pressure & $0.20 \%$ \\
\hline Path length & $0.10 \%$ \\
\hline Volumetric fraction & $1.26 \%$ \\
\hline Fractional transmission of laser light & $0.17 \%$ \\
\hline Absorption coefficient & $1.3 \%$ \\
\hline
\end{tabular}

From the experimentally determined absorption coefficient, the volumetric fractions for the various concentration tests could be determined via Eq. 4.15. The uncertainty of the measured volumetric fraction was determined using the same ANSV/ASME standard as previously mentioned. The partial derivatives used to calculate the total uncertainty of the volumetric fraction are listed in Eq. 4.6 to 4.9 and Eq. 4.16. The uncertainties for the concentration measurements are presented in Chapter 5 along with the results.

$$
\begin{aligned}
\chi_{C H_{4}} & =\frac{-R \cdot T}{P_{A} \cdot L \cdot \sigma_{C H_{4}}} \ln \left(\tau_{R}\right) \\
\frac{\partial \chi_{C H_{4}}}{\partial \sigma_{C H_{4}}} & =\frac{R \cdot T}{P_{A} \cdot L \cdot \sigma_{C H_{4}}{ }^{2}} \ln \left(\tau_{R}\right)
\end{aligned}
$$

$U_{\chi_{\mathrm{CH}_{4}}}=$

$\sqrt{\left(\frac{\partial \chi_{C H_{4}}}{\partial T}\right)^{2}\left(U_{T}\right)^{2}+\left(\frac{\partial \chi_{C H_{4}}}{\partial P_{A}}\right)^{2}\left(U_{P_{A}}\right)^{2}+\left(\frac{\partial \chi_{C H_{4}}}{\partial L}\right)^{2}\left(U_{L}\right)^{2}+\left(\frac{\partial \chi_{C H_{4}}}{\partial \tau_{R}}\right)^{2}\left(U_{\tau_{R}}\right)^{2}+\left(\frac{\partial \chi_{C H_{4}}}{\partial \sigma_{C H_{4}}}\right)^{2}\left(U_{\sigma_{C H_{4}}}\right)^{2}}$

\subsubsection{CCV methodology}

Cross-correlation velocimetry experiments were conducted on two types of gas mixtures, methane in air and methane in helium. The air mixture set flow range was 0.1 to 0.5 SLPM for methane and 0.5 to 1.5 SLPM for air. This corresponded to an exit velocity of 0.005 to $0.17 \mathrm{~m} / \mathrm{s}$. The helium mixture set flow range was 0.1 to 0.5 SLPM for methane and 1.5 to 4.5 SLPM for air. This corresponded to an exit velocity of 0.013 to $0.42 \mathrm{~m} / \mathrm{s}$. Helium was used in some experiments to increase the contrast for Schlieren imaging 
(especially at the low volumetric fractions of methane) and to alter the structural characteristics of the plume.

During data acquisition, the detector analog gains were set manually to $4 \mathrm{~K}$ via the custom built amplifier. The detector signals were sampled (via DAQ PCI 6259) at $1000 \mathrm{~Hz}$ with 16 bit resolution. From simulation and preliminary experimental data, it was found that the sampling frequency of $1000 \mathrm{~Hz}$ was roughly 200 times greater than the highest fluctuation frequency of the plume as seen in the detector signal.

Figure 4.25 shows a schematic of the methodology used to estimate the line flux of methane in the test plumes based on cross-correlation of the path-integrated concentration measurements. The flow chart can be broken into two main sections: measuring the path integrated concentrations for each laser line, and the cross correlation of these two measurement signals to yield a velocity measurement. The raw detector signals $\left(I_{l}\right.$ or $\left.I_{2}\right)$ were normalized using the reference detector signal $\left(I_{\text {ref }}\right)$ to remove laser noise common to each detector and the base power (no absorption) values $\left(I_{1} \text { or } 2 / I_{\text {ref }}\right)_{0}$ were recorded for each detector. From this, the fractional transmission of laser light $\tau$ was calculated and the path length integrated concentration was determined, and cross correlated to extract a time delay.

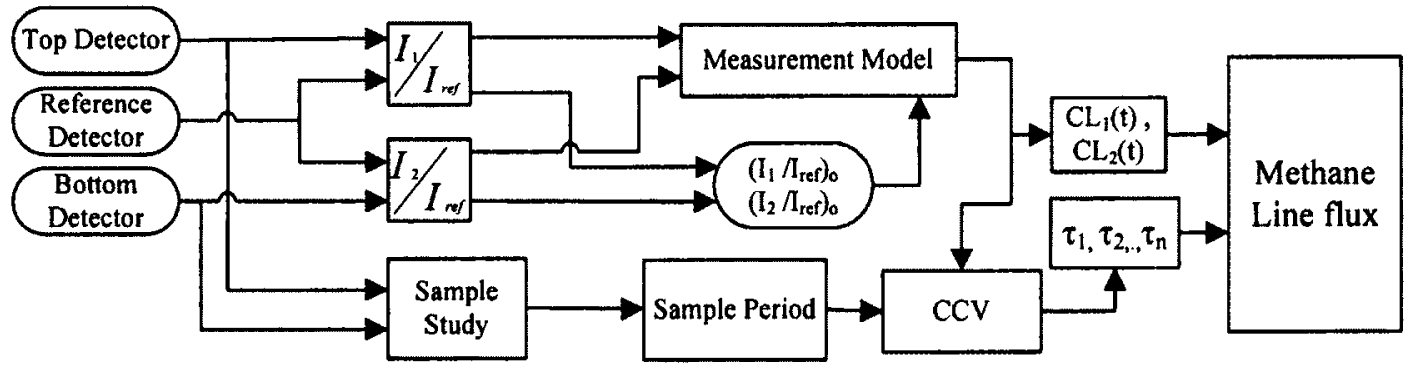

Figure 4.25: The methodology of the experimental validation of $\mathrm{CCV}$ via direct absorption spectroscopy

The measurement model used to infer the path integrated concentration can be seen below.

$$
C L_{C H_{4}}=\frac{-1}{\sigma_{C H_{4}}} \ln \left(\tau_{R}\right)
$$

where

$C L_{\mathrm{CH}_{4}}$ is the path integrated concentration measurement $\left[\mathrm{mol} / \mathrm{m}^{2}\right]$. 
Figure 4.26 and Figure 4.27 shows an example of the cross-correlation of the two time-resolved path integrated concentration signals. The dominant time lag between the two signals occurs where $R_{x y}$ is maximized, corresponding to a time delay of 0.937 seconds (i.e. 937 samples at a sample frequency of $1000 \mathrm{~Hz}$ ). Given the fixed beam separation distance of $39.1 \mathrm{~mm}$, the $0.937 \mathrm{~s}$ time delay equates to an average flow velocity of $0.042 \mathrm{~m} / \mathrm{s}$ (see Eq. 4.19).

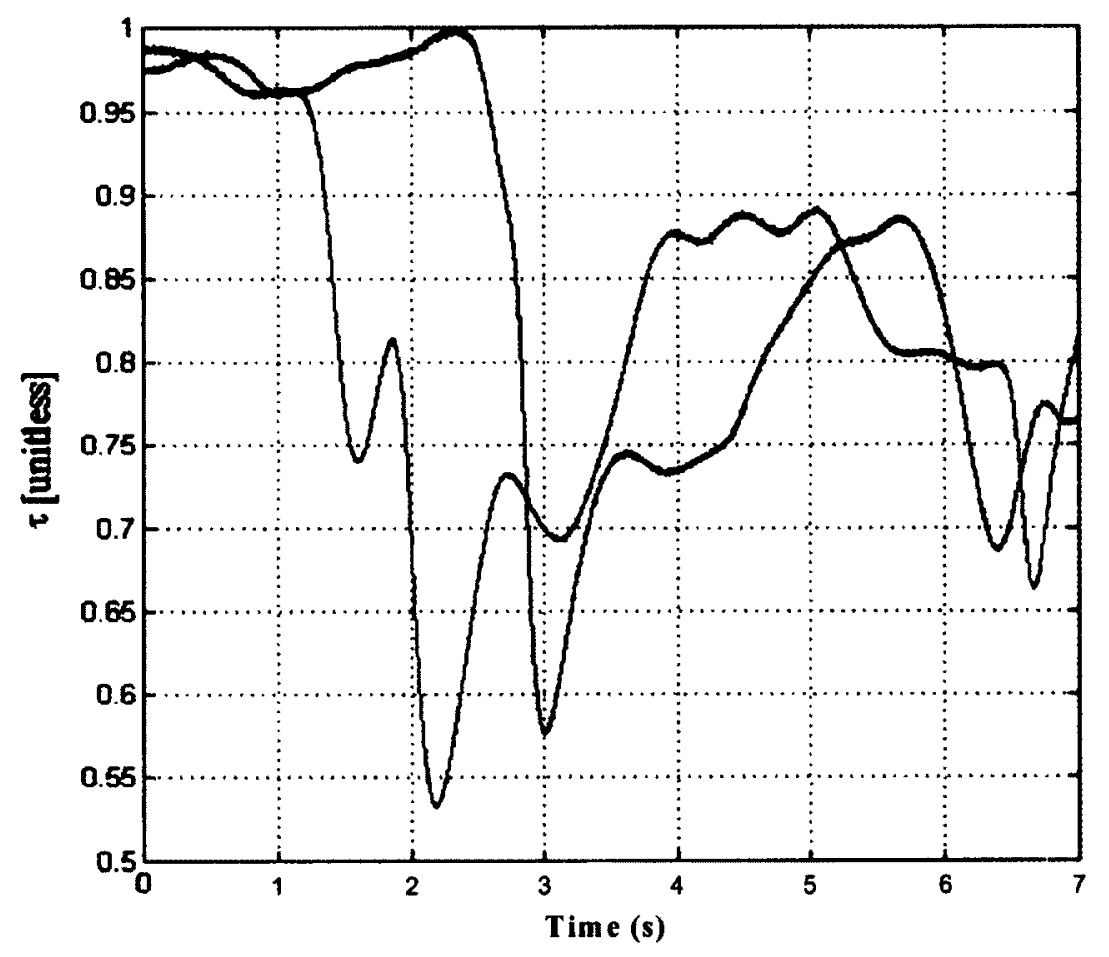

Figure 4.26: Example of $\tau$ for Air -Methane Test 

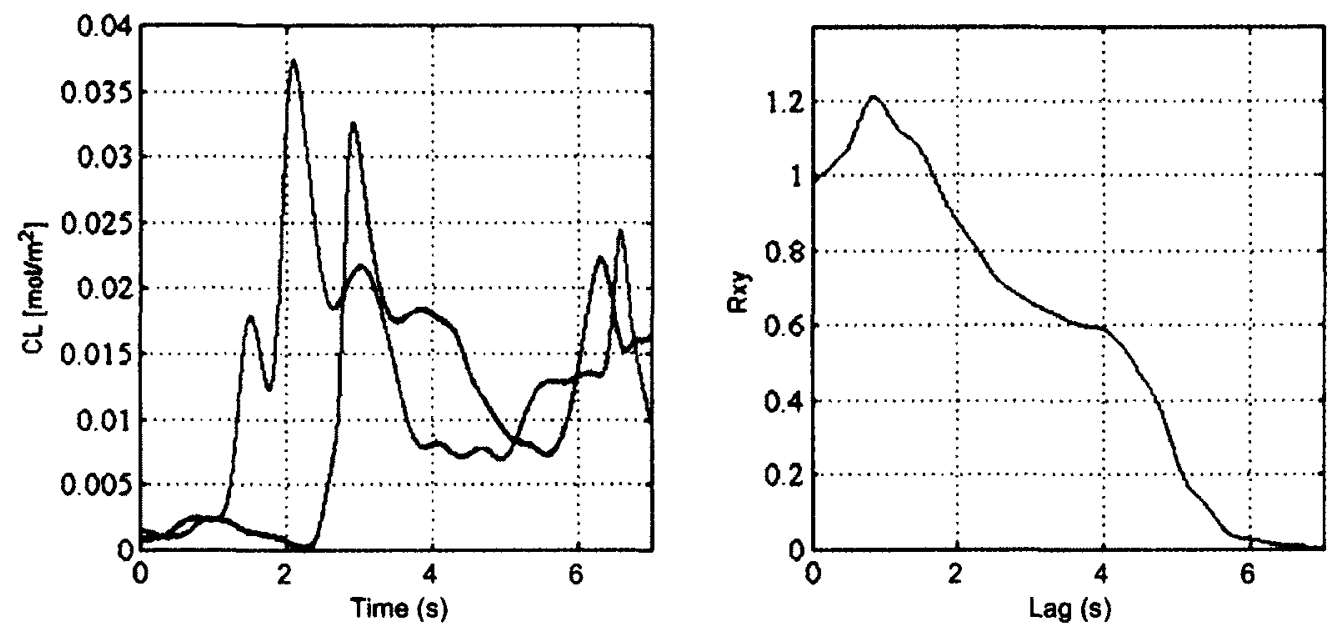

Figure 4.27: Example of calculated CL for both laser/detector lines (Left) and the $R_{x y}$ coefficient (Right)

The precision uncertainty of the velocity was calculated using the TSM method seen in Eq. 4.20. From the measured velocity and path integrated concentration of methane, the measured line flux of methane was calculated using the relation seen in Eq. 4.21, and its associated uncertainty by Eq. 4.22.

$$
\begin{aligned}
& V_{C C V}=\frac{D_{\text {beam }}}{\Delta t_{C C V}} \\
& U_{V_{C C V}}=\sqrt{\left(\frac{1}{\Delta t_{C C V}}\right)^{2}\left(U_{D_{\text {beam }}}\right)^{2}+\left(\frac{D_{\text {beam }}}{\Delta t_{C C V}{ }^{2}}\right)^{2}\left(U_{\Delta t_{C C V}}\right)^{2}} \\
& \dot{m}_{\mathrm{CH}_{4}}{ }^{\prime}=C L_{\mathrm{avg}} \cdot M W_{\mathrm{CH}_{4}} \cdot V_{C \mathrm{CV}} \\
& U_{\dot{m}_{C H_{4}}}=\sqrt{\left(M W_{C H_{4}} \cdot V_{C C V}\right)^{2}\left(U_{C L_{a v g}}\right)^{2}+\left(C L_{a v g} \cdot M W_{C H_{4}}\right)^{2}\left(U_{V_{C C V}}\right)^{2}}
\end{aligned}
$$

where:

$D_{\text {beam }}$ is beam separation distance $[\mathrm{m}]$

$\Delta t_{C C V}$ is the extracted time delay [s]

$m_{\mathrm{CH}_{4}}{ }^{\prime}$ is the line flux of methane $[\mathrm{g} / \mathrm{s}-\mathrm{m}]$

$C L_{\text {avg }}$ is the path integrated concentration measurement $\left[\mathrm{mol} / \mathrm{m}^{2}\right]$

$M W_{C H_{4}}$ is the molecular weight of methane $[\mathrm{g} / \mathrm{mol}]$

$V_{C C V}$ is the plume velocity $[\mathrm{m} / \mathrm{s}]$

$L$ is the plume's characteristic length [m] 


\subsubsection{Mass Flow Transverse test}

To validate the line flux measurements conducted using the novel DAS-CCV technique, a transverse test was conducted to measure the total mass flow rate of the hydrocarbon plume. The plume generator was traversed perpendicularly to the HeNe laser beams in $1 \mathrm{~cm}$ increments over a total path length of $24 \mathrm{~cm}$. A schematic of the traverse test can be seen in Figure 4.28.

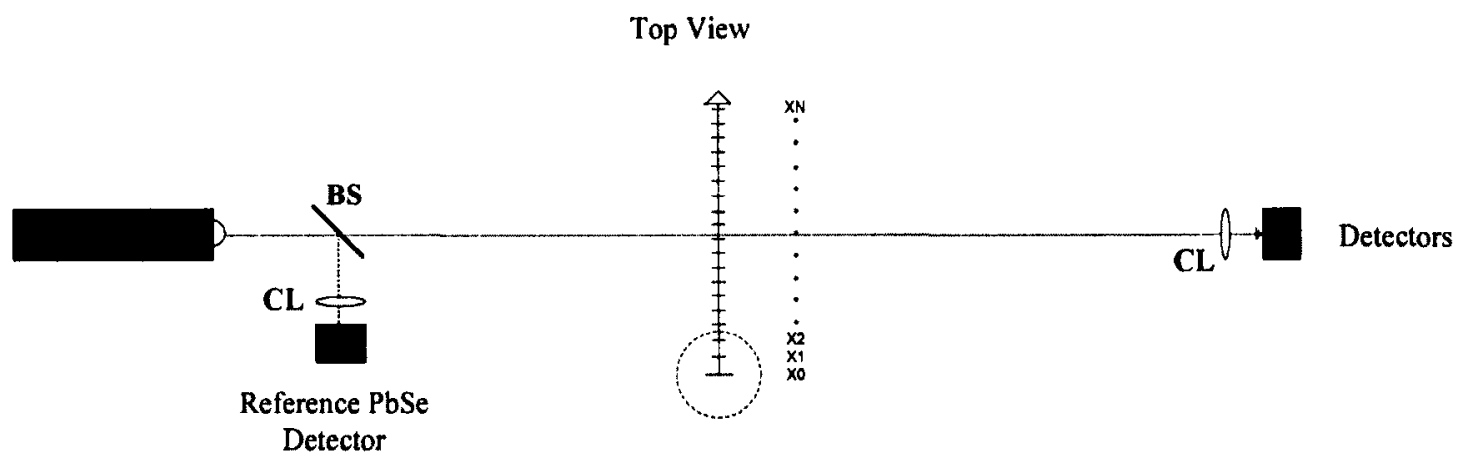

Figure 4.28: Schematic of the traverse test to measure the total mass flow of the plume

From the traversed path integrated concentration measurements, the total mass flow of methane was calculated using Eq. 4.23 seen below.

$$
\dot{m}_{C H_{4}}=\int_{0}^{X} \dot{m}_{C H_{4}}{ }^{\prime} d x=\int_{0}^{X} C L_{\text {avg }} \cdot M W_{C H_{4}} \cdot V_{C C V} d x
$$

where:

$\dot{m}_{\mathrm{CH}_{4}}$ is mass flow of methane $[\mathrm{g} / \mathrm{s}]$

$X$ is the traverse path length [m]

\subsubsection{Schlieren Imaging Methodology}

As stated the earlier the Schlieren imaging system was implemented to gather information of the plume's dynamics and eddy length scales. Measurements were performed using a 100-image data set for each mixture set point. The minimal image pre-processing used was the background removal and the application of a threshold. The background removal was achieved by a simple difference technique that requires a "no plume" image to remove the background and non-uniform lighting from the lighting source. However, due to the innate nature of (a 50\% cut-off) Schlieren image, two forms 
of the differenced image were combined to create the overall plume image. The "differenced" image was converted to binary format by the application of a threshold. This created added ease for batch processing (due to binary format) and edge detection of the plume. An example of the image processing can be seen below in Figure 4.29. The two difference techniques are seen in the two leftmost columns and their corresponding result in the $3^{\text {rd }}$ column. The $4^{\text {th }}$ column is the final image after the binary threshold has been applied, which is then combined as seen in Figure 4.30. The 100 combined binary images were averaged over the set and used to measure the pixel path length of the plume. The conversion to real-world coordinates was done by standard control object calibration technique, where an image of an object of known length was acquired to determine the $x-y$ axis pixel to $\mathrm{mm}$ transformation factor ( $x$-axis: $0.35 \mathrm{~mm} / \mathrm{pixel} ; y$-axis: $0.44 \mathrm{~mm} / \mathrm{pixel}$ ).
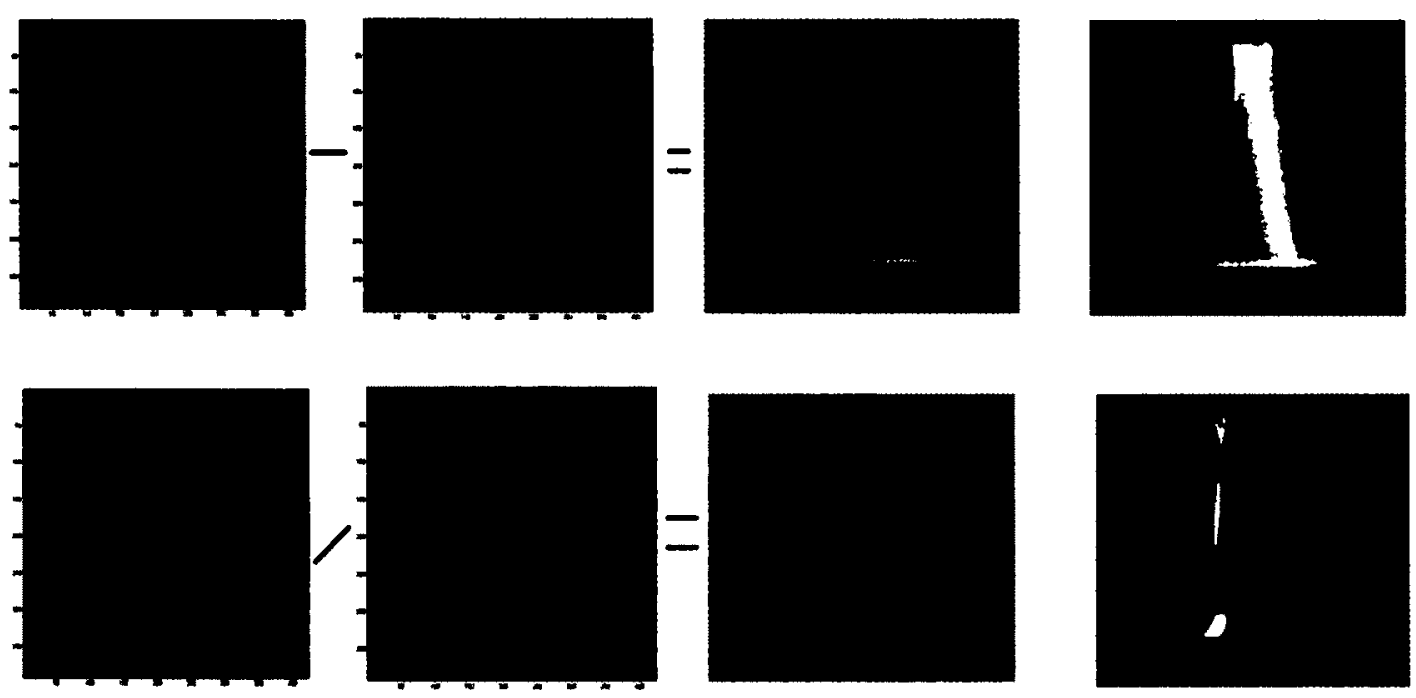

Figure 4.29: Example of the image processing used to gather spatial information of the plume. 


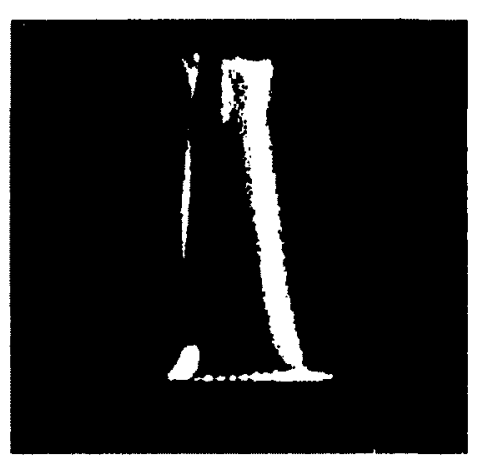

Figure 4.30: Example of the combined binary image used to estimate length scales in the plume 


\section{Chapter 5}

\section{Results}

The primary motivation for this work was to develop a novel, economical, diagnostic technique that has the capacity to measure VOC emissions from LSTs. To quantify the performance of the proposed system, concentration measurement sensitivity, accuracy, and time response were determined in a variety of experiments as presented below.

\subsection{VOC Sensor Response}

\subsubsection{Transient Response}

The transient delay of each detector was measured for a step input of the laser power of 100 to $0 \%$ and $0 \%$ to $100 \%$, by directly segmenting the laser beam circuit. The dominant transient delay is expected from the amplification electronics both from the OP amp and the analog low pass filter added to impede $\mathrm{AC}(60 \mathrm{~Hz})$ noise. Rise time tests were performed at the gain settings of $4 \mathrm{~K}$ and $5 \mathrm{~K}$, with a sampling frequency of $10 \mathrm{kHz}$. The average rise times for both detector assemblies were found to be 0.0013 seconds, implying an inherent cut-off frequency of $\sim 770 \mathrm{~Hz}$. From this it was determined that the maximum resolvable vertical velocity fluctuation for the experimental beam spacing of $39.1 \mathrm{~mm}$ was $\sim 30 \mathrm{~m} / \mathrm{s}$, and the optimal sampling frequency was $1000 \mathrm{~Hz}$. The average transient response of each detector is tabulated in Table 5.1, and a test sample can be seen Figure 5.1.

Table 5.1: Detector Assemblies transient response characteristics

\begin{tabular}{|c|c|c|c|}
\hline Detector & $\begin{array}{c}\text { Rise } \\
\text { Time (s) }\end{array}$ & $\begin{array}{c}\text { Settling } \\
\text { Time (s) }\end{array}$ & $\begin{array}{c}\text { Overshoot } \\
\text { (\%) }\end{array}$ \\
\hline Top & 0.0014 & 0.0025 & 0.0316 \\
\hline Bottom & 0.0013 & 0.0028 & 0.0335 \\
\hline
\end{tabular}




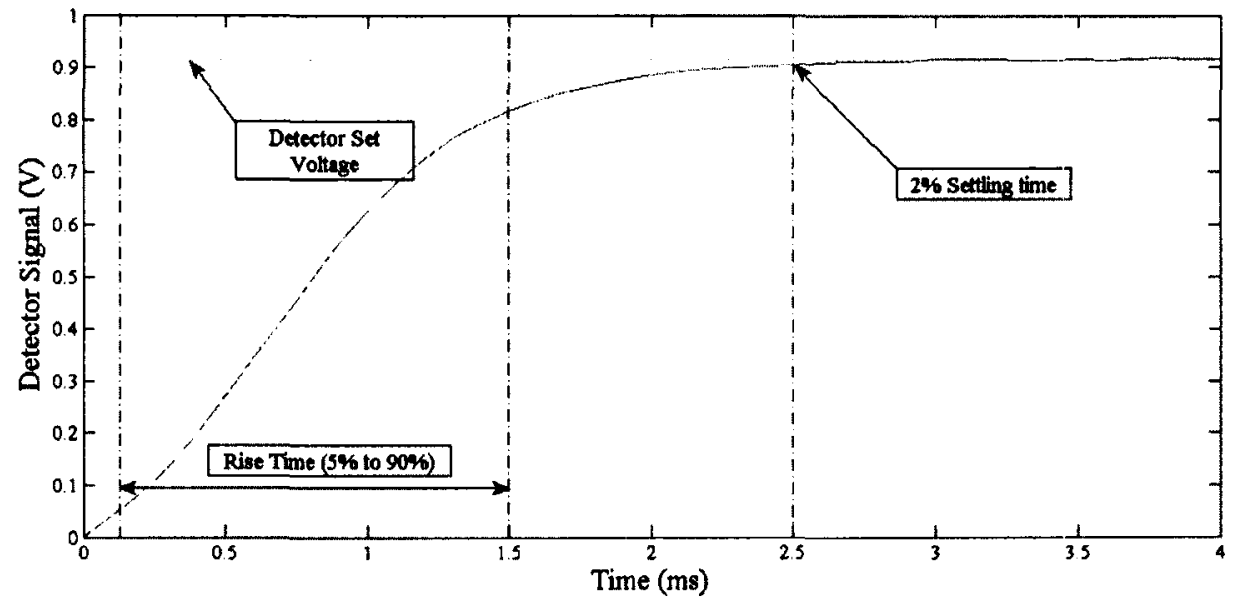

Figure 5.1: Detector response to a step change in incident laser radiation from 0 to $100 \%$ of the laser power

\subsubsection{Random Noise Distribution}

The frequency response (i.e. power spectrum) of the detector signal, sampled at $1000 \mathrm{~Hz}$ is shown in Figure 5.2, which shows that the VOC measurement system is still plagued by $1 / \mathrm{f}$ noise. However, the figure shows that the magnitude of the combined (laserdetector-amplifier) noise profiles are $\sim 4$ orders of magnitude lower that the DC power signal available for absorption. The normalized average noise values using the laser reference detector for the $1^{\text {st }}$ amplification stage were found to be $0.1 \%$ and $0.095 \%$ for the two laser-detector pairs. The $2^{\text {nd }}$ and $3^{\text {rd }}$ stages of the custom amplifier were designed to segregate the DC signal and provide $\mathrm{AC}$ amplification with the intent of providing a higher signal to sample range ratio (of the acquisition electronics) for the alternating component of the absorption signal. While the $2^{\text {nd }}$ and $3^{\text {rd }}$ stages satisfy their primary purpose, they do not provide a higher SNR than the $1^{\text {st }}$ stage. The normalized average noise values using the laser reference detector were found to be $0.11 \%$ and $0.097 \%$ for the $2^{\text {nd }}$ stage, and $0.13 \%$ and $0.099 \%$ for the $3^{\text {rd }}$ stage. Additionally, the power spectrum of the detector frequency response illustrates that the shielding implemented on the electronics design does not fully prevent the introduction of $\mathrm{AC}(60 \mathrm{~Hz})$ noise and its $n$ order harmonics (i.e. $120 \mathrm{~Hz}, 180 \mathrm{~Hz}, 240 \mathrm{~Hz}$ etc.). This is seen as small spikes in power spectrum at the aforementioned frequencies highlighted with dashed red boxes in Figure 5.2. From these results, it can be seen that additional optimization could be attempted to reduce the $1 / \mathrm{f}$ noise and $\mathrm{AC}$ noise contributors. 

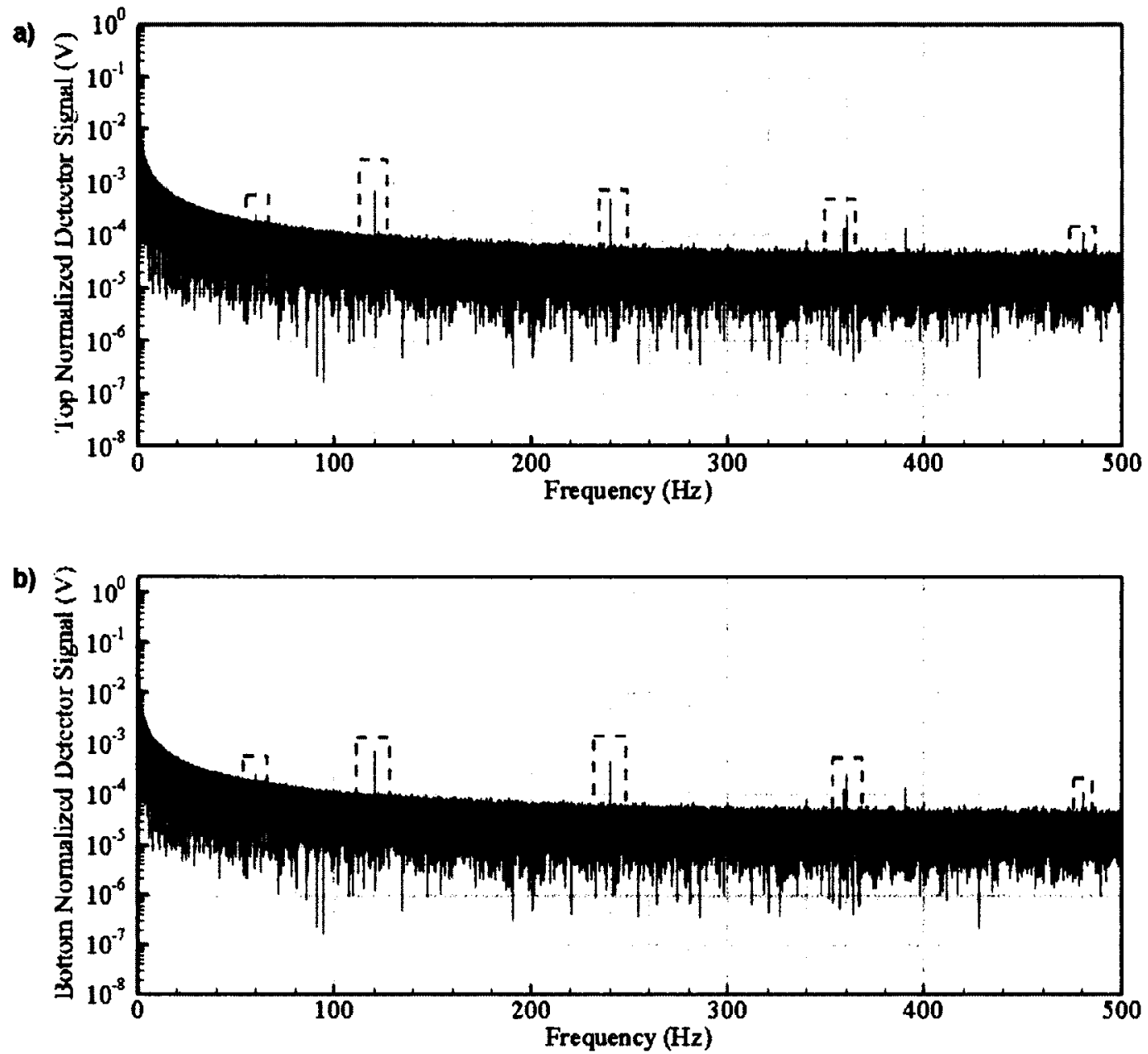

Figure 5.2: Power spectrum distribution of the raw signals of (a) top detector and

(b) bottom detector; The AC $(60 \mathrm{~Hz})$ noise and its $n$ order harmonics are highlighted in dashed red boxes

From further investigation, it was determined that the $\mathrm{AC}$ noise contributions, were inherent to the laser emission signal, as seen in Figure 5.3. Here the detector's power spectrum is presented both using and not using the reference detector's signal for additional conditioning. The reference detector was initially implemented due to the laser noise specifications of $\pm 5 \%$, however, from experimentation it was found that after reaching steady state operation $(\sim 2-4$ hours), the laser noise was significantly lower across the DC $-500 \mathrm{~Hz}$ bandwidth and was only seen at the $n$ harmonics of $60 \mathrm{~Hz}$. The low noise behaviour of the HeNe laser makes it a viable lasing source for the full scale VOC sensor. 
Additionally, from the same technique it was determined that the $1 / \mathrm{f}$ noise source contributor is either the detector and/or the amplifier assembly; however, quantification of the exact contributor to the $1 / \mathrm{f}$ noise would require additional design and benchmarking with secondary components (i.e. commercial amplifiers with detailed noise characteristics). It should be noted that the use of the specified detectors and the custom amplifier is still warranted under the achievable cost-effective sensitivity, discussed in Section 5.2, since the measured noise $(0.1 \%)$ yields sufficient concentration sensitivity.

a)

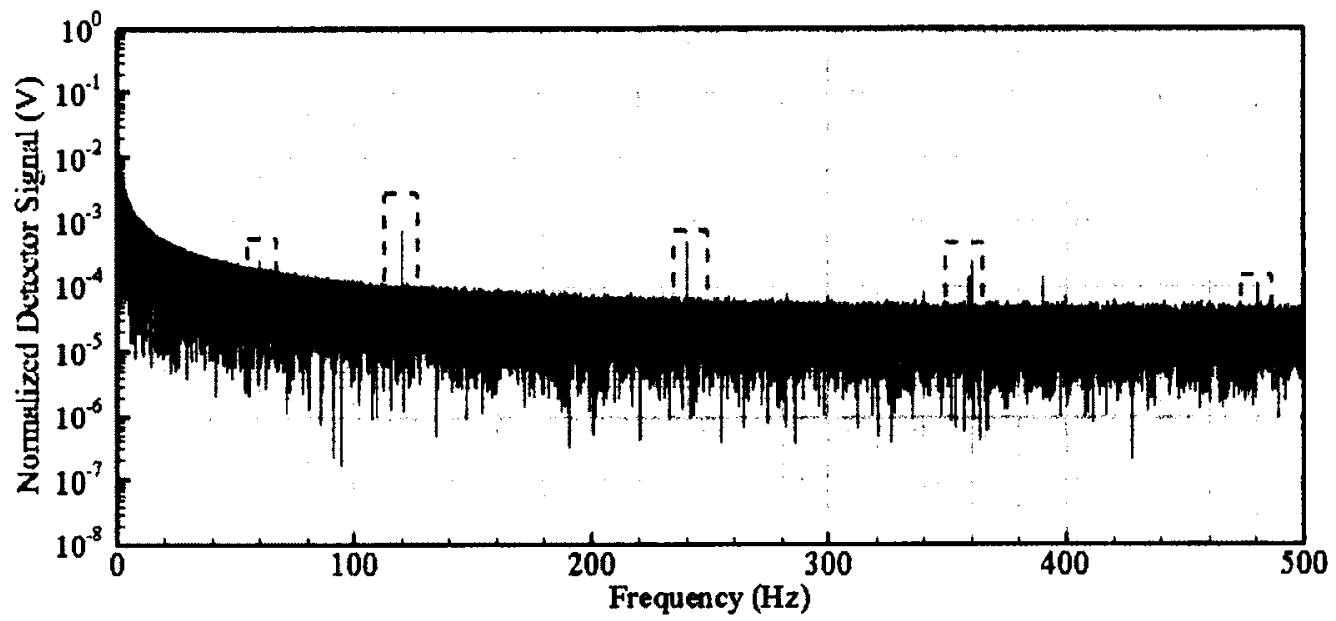

b)

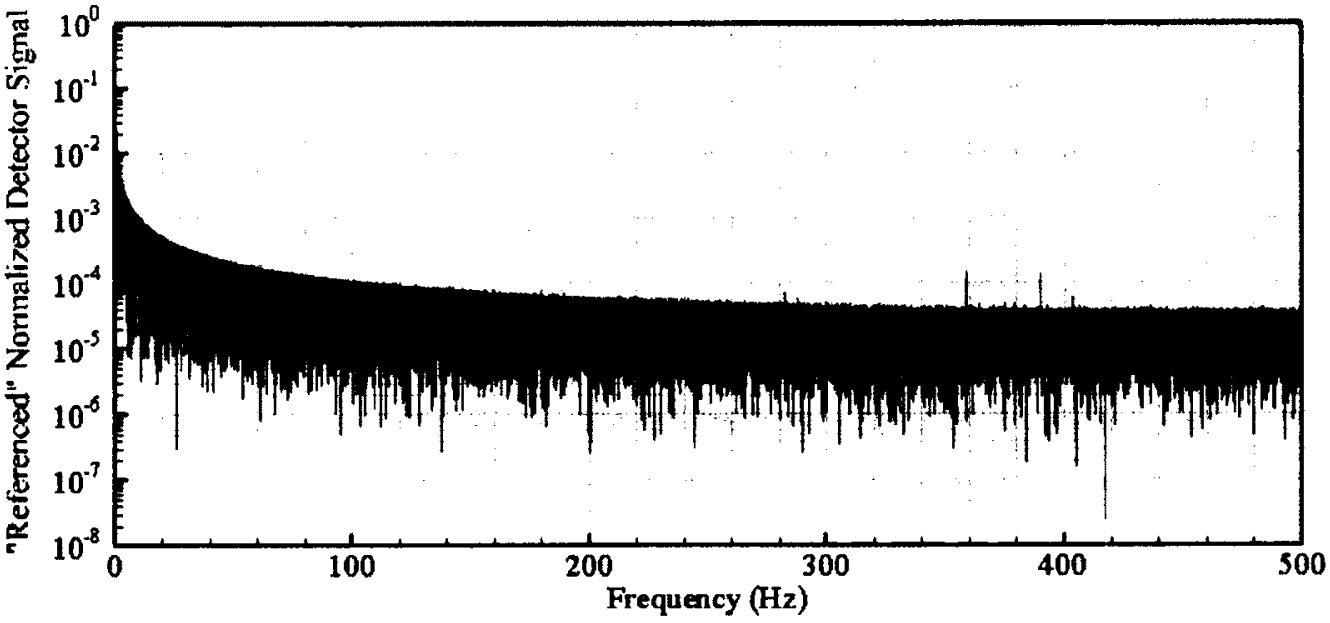

Figure 5.3: (a) Power spectrum of the detector signal; (b) Power spectrum of the normalized detector signal. The AC $(60 \mathrm{~Hz})$ noise and its $n$ order harmonics are highlighted in dashed red boxes 


\subsection{Concentration Measurement Sensitivity}

To test the concentration sensitivity of the in-lab VOC measurement system, experimental data were gathered from a variety of concentration mixtures of air- $\mathrm{CH}_{4}$ using the flow-through cell version of the experimental apparatus, as described in Section 4.1. The transmissivity was calculated by measuring the base line power (no absorption) and the power attenuation for each test concentration. Combined with the measured gas conditions, i.e. barometric pressure and temperature, the volumetric fraction of methane $\left(\chi_{\mathrm{CH}_{4}}\right)$ was inferred using the Beer-Lambert law, as presented in Eq. 4.15.

Figure 5.4 shows the measured $\chi_{\mathrm{CH}_{4}}$ for two step-input tests. In total, 20 step input concentration tests were conducted. By comparing the transient response of the detector and the transient delay of the concentration measurements, the transient delays observed in Figure $5.4 \mathrm{a}, \mathrm{b}$ are (99.98\%) due to the transient response of the MFCs. The tests showed good concentration sensitivities with very small error $(0.5 \%$ noise). The path-integrated volume fraction sensitivity in $\mathrm{ppm} \cdot \mathrm{m}$ for each test was calculated based on the $10 \mathrm{~mm}$ path length of the flow through cell. The path length integrated sensitivity is useful to compare measurements conducted across different path lengths especially considering that the future VOC sensor laser path lengths could be in the range of 5$30 \mathrm{~m}$. The time averaged sensitivities for a $300 \mathrm{ppm} \cdot \mathrm{m}$ test are tabulated in Table 5.2 . Due to the accuracy limits of the MFCs, tests conducted at lower concentrations than $250 \mathrm{ppm} \cdot \mathrm{m}(0.025 \%$ within the flow through cell) were found to incur greater random error in the measurements.

Table 5.2: Time averaged concentration sensitivity

\begin{tabular}{|c|c|}
\hline Time average Span (s) & Random Error (\%) \\
\hline-- & 0.5 \\
\hline 1 & 0.11 \\
\hline 10 & 0.08 \\
\hline 15 & 0.039 \\
\hline
\end{tabular}



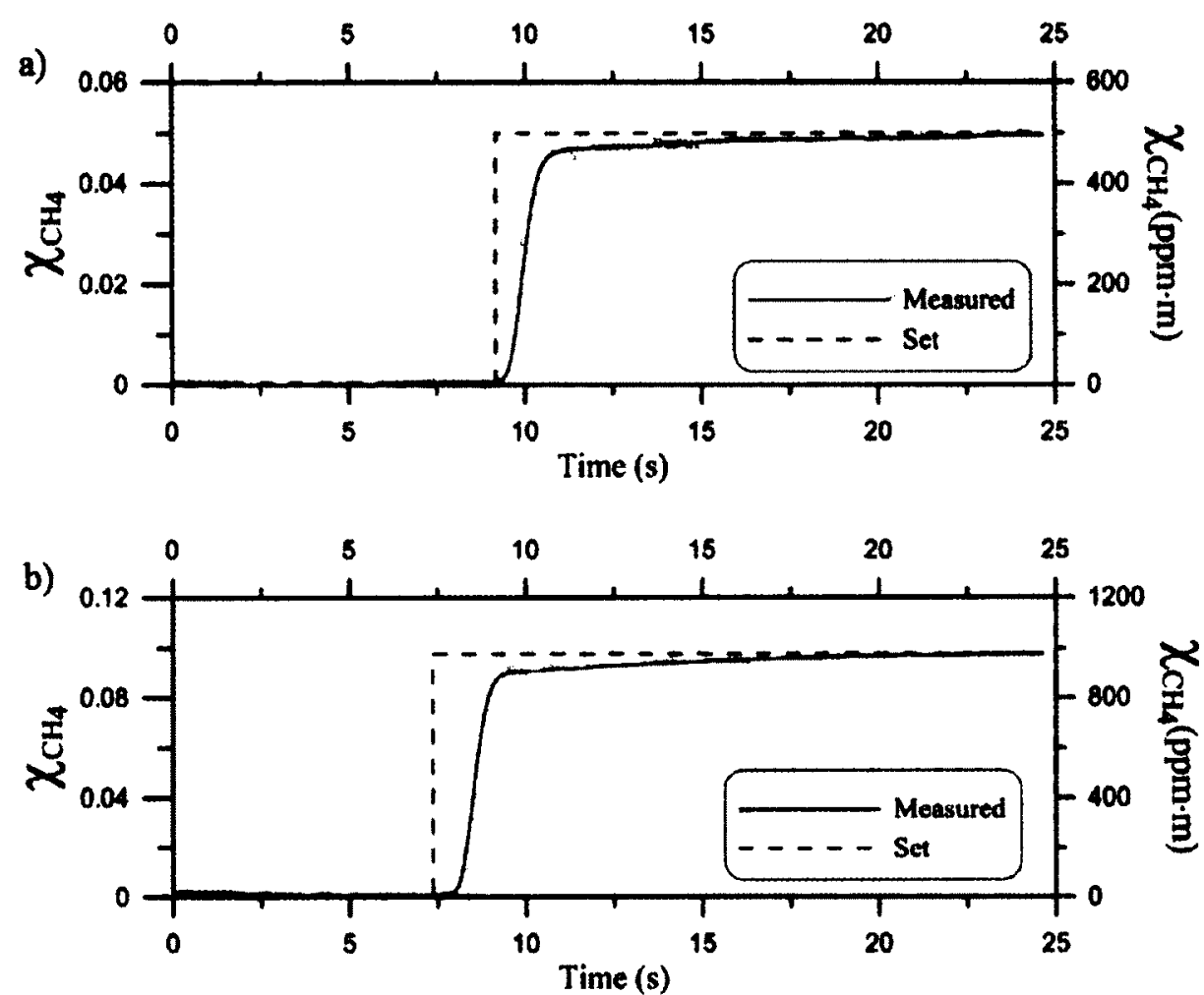

Figure 5.4: Measurements of the $\chi_{\mathrm{CH}_{4}}$ for step inputs of 0 to (a) 0.05 and (b) 0.1

Figure 5.5 shows the measured $\chi_{\mathrm{CH}_{4}}$ for a prolonged multi-step input test from 0.05 to 0.1 . From the plot, the VOC sensor's concentration tracking was found to be quite accurate with minimal (random) error of approximately $0.5 \%$ or less. Taking this into account including the drift noise measured from prolonged $(1 \mathrm{hr}) 0 \%$ methane tests, which found that the average deviation due to drift was $1.12 \%$, the combined noise (added in quadrature) was $1.27 \%$. This yields an overall SNR of 81.5 and a theoretical lower detection limit $(\mathrm{SNR}=1)$ of $2.22 \mathrm{ppm} \cdot \mathrm{m}$. As mentioned in Section 2.3.1, from the measurements conducted by Chambers (2004), in-field concentrations are expected to be approximately 2-3 times the ambient concentrations of methane. Assuming a path length range of 5 to $90 \mathrm{~m}$ (corresponding to the EFRT tank diameter) and equally distributed concentration of methane (among other hydrocarbons), the theoretical concentration detectivity limit would range from $0.44 \mathrm{ppm}$ to $0.02 \mathrm{ppm}$. Considering, the minimum concentration of methane in air is at background levels $(1.7 \mathrm{ppm})$ the lab prototype was found to have satisfactory sensitivity for a future field application. 


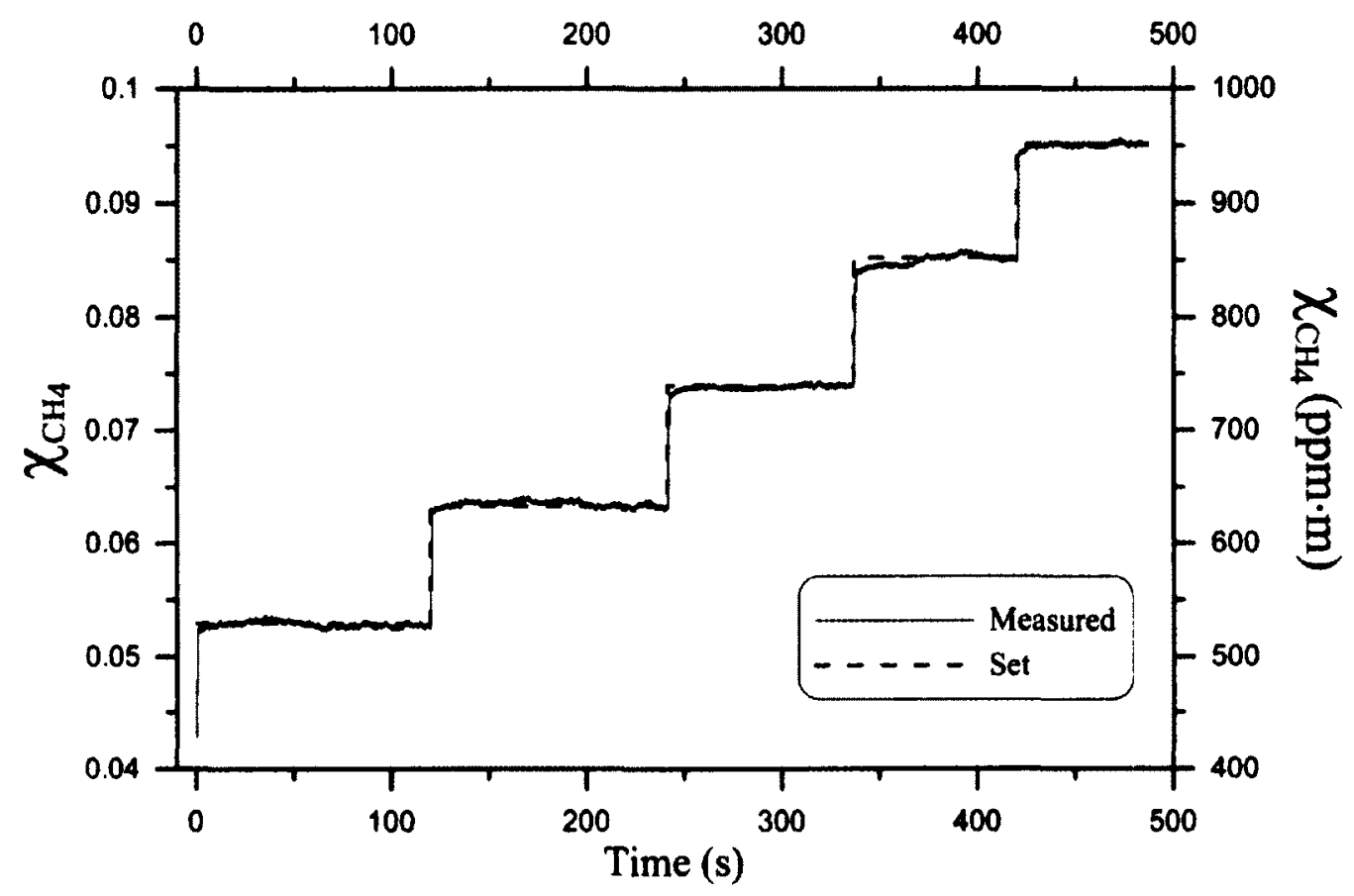

Figure 5.5: Measurements of the $\chi_{\mathrm{CH}_{4}}$ of a Multi-Step concentration tests from 0.05 $-0.95$

The uncertainty of the measured $\chi_{\mathrm{CH}_{4}}$ presented in Figure 5.6, was calculated from the ASTM TSM method outlined in Eq. 4.17, which combines estimated bias and measured precision uncertainties of the various measurements (temperature, pressure, etc.) in quadrature (Coleman \& Steele, 2009). For each concentration set point, the uncertainty was calculated using a sample set of 60,000 and a t-value of 1.96 (corresponding to the $95 \%$ confidence interval) to calculate the precision uncertainty of the fractional transmission $\tau$ and the temperature as seen in Eq. 4.5. The first 500010000 test samples were omitted (before recording the required 60,000 samples), due to the transient delays of the MFCs. Relative contributions to the total uncertainty are tabulated in Table 5.3, where the majority of the measured $\chi_{\mathrm{CH}_{4}}$ uncertainty comes from the absorption coefficient (determined though in-lab calibration). Figure 5.7 shows that the measured $\chi_{\mathrm{CH}_{4}}$ uncertainty displayed negligible growth as the set concentration of methane was lowered, which infers good $(\sim 1.35 \%)$ precision uncertainties at lower concentrations. 


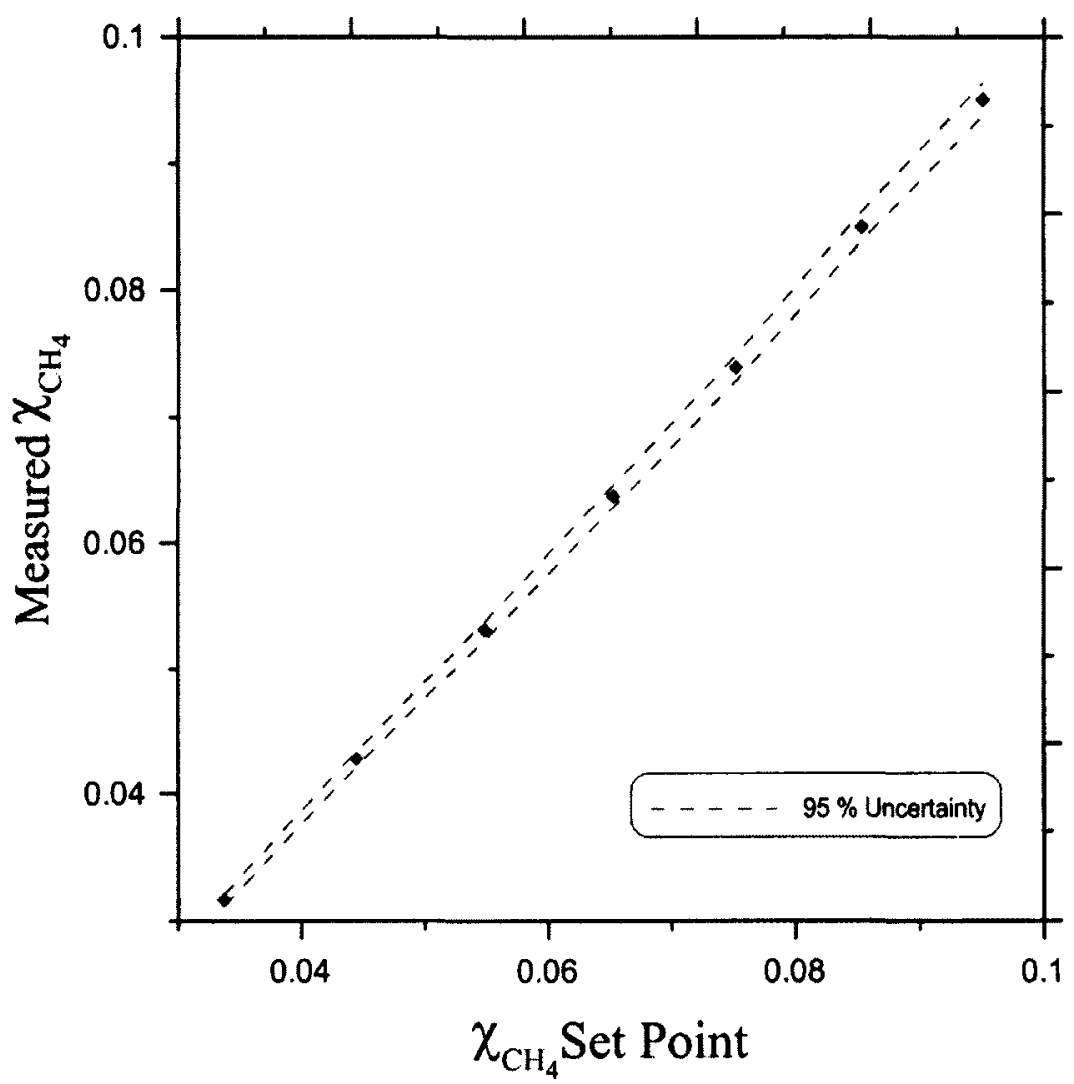

Figure 5.6: Measured vs. Set $\chi_{\mathrm{CH}_{4}}$ along with the associated measurement uncertainty

Table 5.3: Relative uncertainties of the measured/calculated parameters

\begin{tabular}{|c|c|}
\hline Constituent & Relative Uncertainty \\
\hline Temperature & $0.06 \%$ \\
\hline Pressure & $0.20 \%$ \\
\hline Path length & $0.10 \%$ \\
\hline Absorption coefficient & $1.30 \%$ \\
\hline Fractional transmission of laser light & $0.17 \%$ \\
\hline Volumetric fraction & $1.35 \%$ \\
\hline
\end{tabular}




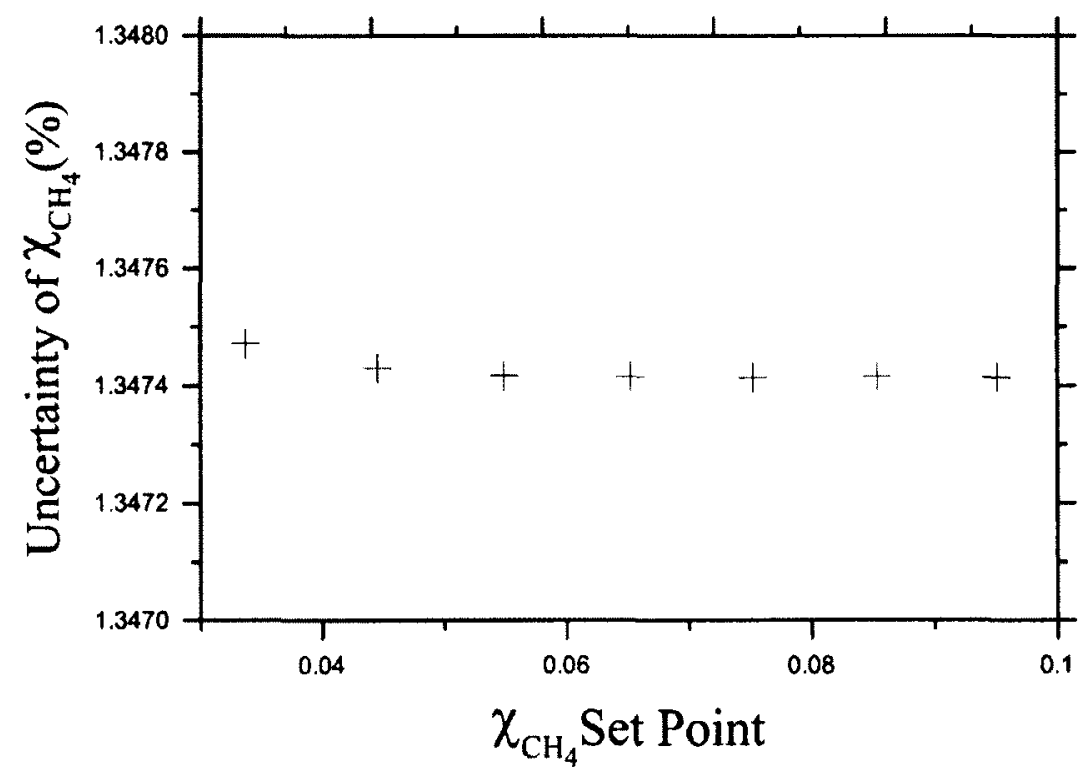

Figure 5.7: Relative measured uncertainty as a function of set $\chi_{\mathrm{CH}_{4}}$

\subsection{CCV and Methane Flux Measurements}

The cross-correlation velocimetry technique was tested on two sets of mixtures, air- $\mathrm{CH}_{4}$ and helium- $\mathrm{CH}_{4}$ as summarized in Table 5.4. The objective of these experiments was to utilize cross-correlation of the two vertically displaced path length integrated concentration measurements to estimate the local line flux of a diluted methane plume. These experiments were conceived as proof-of-concept tests of a potential future multiline grid-based VOC sensor to evaluate its ability to measure the mass flux of a methane plume traversing through a laser grid.

Table 5.4: CCV Experimental Study Breakdown

\begin{tabular}{|c|c|c|}
\hline Diluting Gas & Diluting Gas (SLPM) & Methane (SLPM) \\
\hline Air & 1.5 & $\left.\begin{array}{lllllll}0.1 & 0.2 & 0.3 & 0.4 & 0.5\end{array}\right]$ \\
\hline Air & 1.0 & {$\left[\begin{array}{llllll}0.1 & 0.2 & 0.3 & 0.4 & 0.5\end{array}\right]$} \\
\hline Air & 0.5 & {$\left[\begin{array}{lllll}0.1 & 0.2 & 0.3 & 0.4 & 0.5\end{array}\right]$} \\
\hline Helium & 1.5 & {$\left[\begin{array}{lllll}0.1 & 0.2 & 0.3 & 0.4 & 0.5\end{array}\right]$} \\
\hline Helium & 3.0 & {$\left[\begin{array}{llllll}0.1 & 0.2 & 0.3 & 0.4 & 0.5\end{array}\right]$} \\
\hline Helium & 4.5 & {$\left[\begin{array}{lllll}0.1 & 0.2 & 0.3 & 0.4 & 0.5\end{array}\right]$} \\
\hline
\end{tabular}


Considering the range of experimental flow rates an diameter of the plume generator, Froude numbers (calculated using Eq. 5.1) for the plume should range between 0.01-0.04 (for the helium- $\mathrm{CH}_{4}$ mixtures) and 10-28 (for the helium- $\mathrm{CH}_{4}$ mixtures). This infers that the helium- $\mathrm{CH}_{4}$ plume motion will be buoyancy dominated and have a high fluctuation (or pulsation) frequency ( $\propto \mathrm{VD} / \mathrm{Fr}^{38}$ ), while the Air- $\mathrm{CH}_{4}$ plume motion will be less dominated by buoyant effects and will have lower fluctuation frequencies in the flow (Mell et al., 1996).

$$
F r=\frac{v}{\left(\frac{\Delta \rho}{\rho_{0}} g \cdot D\right)^{1 / 2}}
$$

where:

$\mathrm{V}$ is the exit velocity $[\mathrm{m}]$,

$\Delta \rho$ is the density difference to the surroundings $\left[\mathrm{kg} / \mathrm{m}^{3}\right]$,

$\rho_{o}$ is the reference density $\left[\mathrm{kg} / \mathrm{m}^{3}\right]$,

$g$ is acceleration due to gravity $\left[\mathrm{m} / \mathrm{s}^{2}\right]$, and

$\mathrm{D}$ is the exit diameter of the plume generator [m].

\subsubsection{Path integrated concentration measurements}

Figure 5.8 shows the mean path intergrated concentration $(C L)$ calculated by Eq. 4.18 from the fractional transmission of laser light through the diluted methane mixtures. The $95 \%$ uncertainties of the mean $C L$ measurements were calculated (as seen in Eq. 4.5) using a sample set of 60000 and with a student distribution t-value of 1.96. The uncertainty values are tabulated in Table 5.5. The plot of the mean path integrated concentration shows good linear agreement with the set methane mass flow; however, the slope is inconsistent between the two flow mixtures (air \& helium) for the same set methane mass flow rate. This is attributed to the fluctuation magnitude and frequency of the fluctuations of both mixtures. The air- $\mathrm{CH}_{4}$ flows induce high magnitude, lower frequency fluctuations in the $C L$ measurements, whereas the helium-methane mixtures induce smaller magnitude, higher frequency eddies. 
Table 5.5: The relative uncertainties of the mean path integrated concentration of methane

\begin{tabular}{|c|c|c|c|c|c|c|}
\hline \multirow{2}{*}{$\begin{array}{c}\text { Methane } \\
\text { Flow rate } \\
\text { (SLPM) }\end{array}$} & \multicolumn{2}{|c|}{ Air Dilution Flow rate } & \multicolumn{3}{c|}{ Helium Dilution Flow rate } \\
\cline { 2 - 7 } & $\begin{array}{c}\mathbf{0 . 5} \\
\text { SLPM }\end{array}$ & $\begin{array}{c}\mathbf{1 . 0} \\
\text { SLPM }\end{array}$ & $\mathbf{1 . 5}$ SLPM & $\begin{array}{c}\mathbf{1 . 5} \\
\text { SLPM }\end{array}$ & $\mathbf{3 . 0}$ SLPM & $\begin{array}{c}4.5 \\
\text { SLPM }\end{array}$ \\
\hline 0.1 & $0.55 \%$ & $0.39 \%$ & $0.42 \%$ & $0.49 \%$ & $0.42 \%$ & $0.46 \%$ \\
\hline 0.2 & $0.51 \%$ & $0.58 \%$ & $0.42 \%$ & $0.47 \%$ & $0.43 \%$ & $0.45 \%$ \\
\hline 0.3 & $0.49 \%$ & $0.50 \%$ & $0.44 \%$ & $0.43 \%$ & $0.53 \%$ & $0.50 \%$ \\
\hline 0.4 & $0.56 \%$ & $0.45 \%$ & $0.41 \%$ & $0.51 \%$ & $0.47 \%$ & $0.44 \%$ \\
\hline 0.5 & $0.51 \%$ & $0.47 \%$ & $0.43 \%$ & $0.46 \%$ & $0.42 \%$ & $0.40 \%$ \\
\hline
\end{tabular}

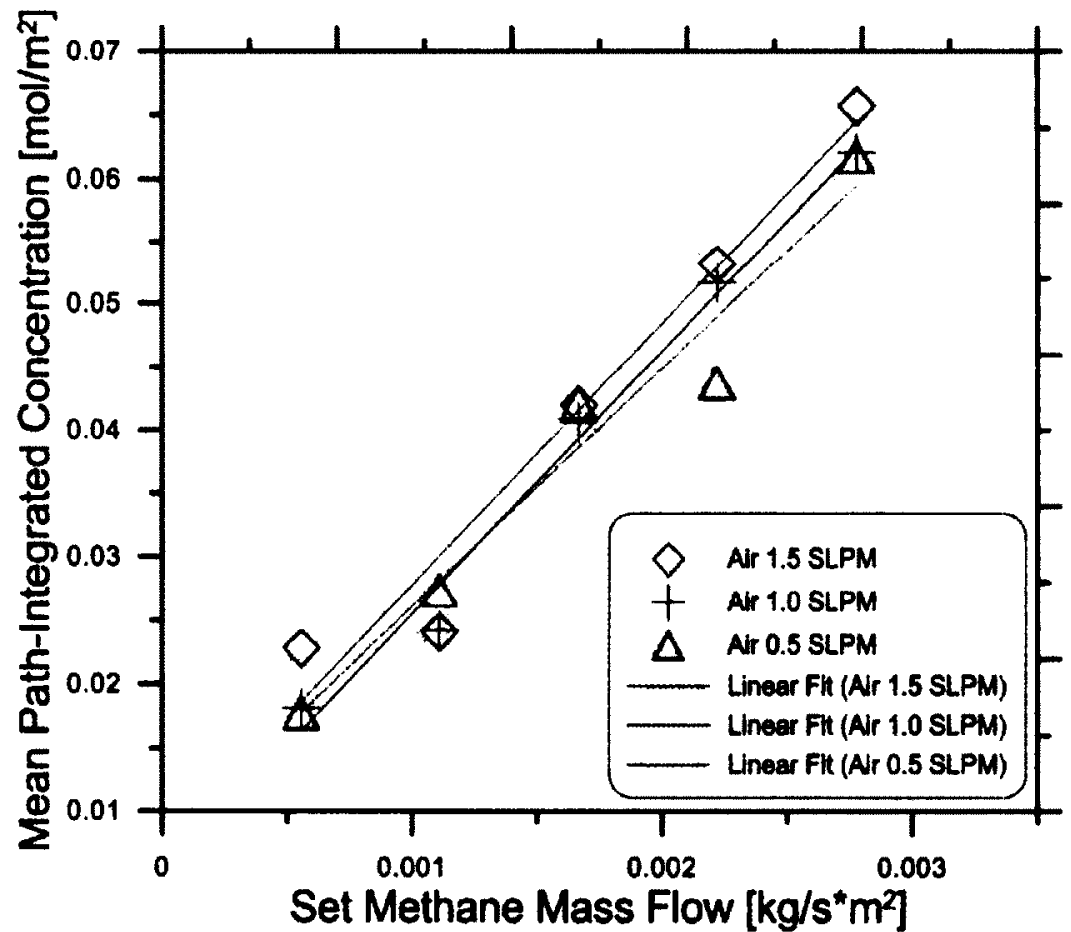

Figure 5.8: Measured path integrated concentration of methane for $\mathrm{Air}-\mathrm{CH}_{4}$ case study 


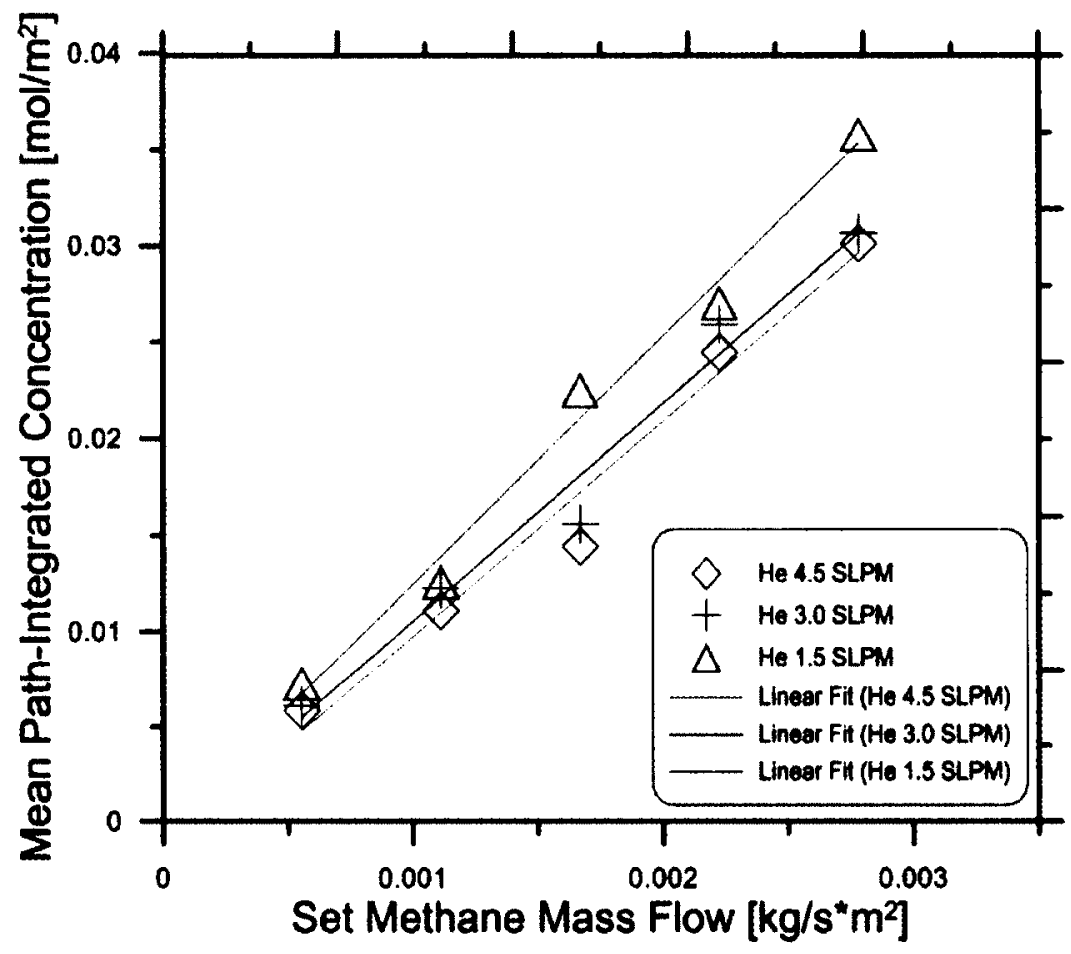

Figure 5.9: Measured path integrated concentration of methane for the Helium- $\mathrm{CH}_{4}$ case study

\subsubsection{CCV}

As stated in Section 3.1.2, the main parameters that affect the cross correlation function are: turbulent eddy behaviour, laser beam separation, measurement noise, sampling frequency and sampling period. The turbulent eddy behaviour of the plume was an uncontrolled function of the buoyant properties of the set mixture flow. The laser beam separation was kept to a minimum of $39.1 \mathrm{~mm}$, based on the minimal achievable spacing between commercially available rigid Mid-IR optical components with protective enclosures. The measurement noise parameters were determined by the economical amplifier design as presented in Section 5.1.2. The sampling frequency was selected based on the transient response behaviour of the detector and amplifiers, presented in Section 5.1 where the cut-off frequency was determined. The cut-off frequency determines the optimal point where a lower frequency results in a loss of information and a higher frequency results in no gain of information for the additional resources required (i.e. buffer memory, computational effort etc..). 


\subsubsection{CCV Sample period}

The sample period, (i.e. the number of samples of each signal that is used to calculate the $R_{x y}$ coefficient), required tuning. In thermocouple-based $\mathrm{CCV}$ in a turbulent fire, Rockwell (2009) demonstrated that the standard deviation of the velocity measured exponentially decayed after the critical sample period was reached. Therefore, the standard deviation of the measured velocity was utilized in tuning the sample period for the DAS CCV.

Figure 5.10 shows the exponential decay of the standard deviation of measured velocity for the maximum (Figure 5.10a) and minimum (Figure 5.10b) experimental flow range of the air- $\mathrm{CH}_{4}$ test cases. The general exponential decay is observed in the higher methane flow set point ( 0.5 SLPM). The lower methane set point (0.1 SLPM) shows more unsteady decay behaviour after $\sim 6000$ samples. This behaviour is attributed to bi-modal fluctuations generally seen in the lower flow regions as discussed in Section 5.3.2.2. For the air- $-\mathrm{CH}_{4}$ mixtures, the sample period of 5000 samples was selected as the optimal point due to behaviour of the standard deviation of error and due to the diminishing improvement for the additional computational effort. This also implies that the VOC sensor could update the velocity measurement at $0.2 \mathrm{~Hz}$. The plot also shows that the overall standard deviation of the error is inversely related to the methane flow rate, such that higher methane flow rates result in a lower variation of the measured velocity. This trend was expected due to the higher absorption from the gas plume. Since the CCV algorithm relies on the turbulent fluctuations to infer the velocity of the plume, the higher methane mole fraction results in a higher fluctuation signal from the combination of larger buoyant eddies and higher absorption. This trend is also discernible between high methane set point (0.5 SLPM) for the two (2) different air set points of (a) 0.5 SLPM and (b) 1.5 SLPM. While the eddy size for the two flows are roughly the same (see section 5.4.1 for further illustration), the absorption signal is higher for the lower set point of air ( 0.5 SLPM) because the volumetric fraction of methane is higher. 


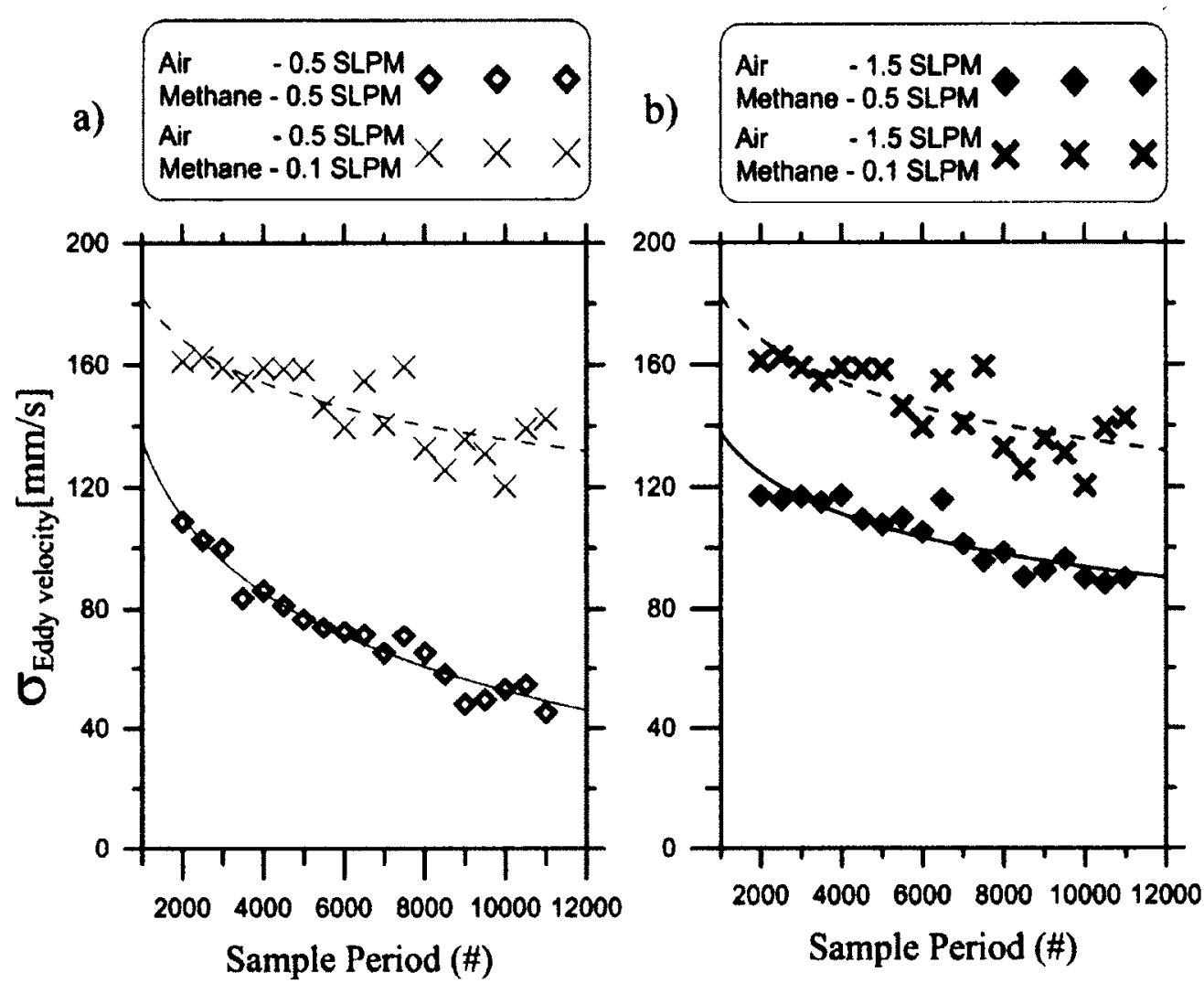

Figure 5.10: Standard deviation of the measured velocity vs. the CCV sample period for Air- $\mathrm{CH}_{4}$ mixtures at the (a) minimum and (b) maximum of experimental air flow range

Figure 5.11 shows the exponential decay of the standard deviation of $\mathrm{CCV}$ measured velocity for the minimum (Figure 5.11a) and maximum (Figure 5.11b) range of helium test cases. The general exponential decay and (slightly) unsteady behaviour can be seen at the low helium flows (1.5 SLPM); however, the higher helium flows (4.5 SLPM) shows greater unsteady settling behaviour, which can be attributed to the higher frequencies observed in the concentration measurements. Unlike the air- $\mathrm{CH}_{4}$ data, the helium data, displayed high-frequency fluctuations and also showed more repetitive behaviour, yielding relatively fewer "unique" fluctuations by which to cross correlate. Additionally, the high diffusivity of helium, discussed in Section 5.4.1, further degrades the velocity measurement, yielding unstable decay behaviour. Therefore, the longer sample runs incurred greater error from misidentification (in the statistical sense) of corresponding fluctuations between the two concentration measurements. To avoid incurring greater error from these misidentifications while maintaining a reasonable 
update frequency, a sample period of 4000 samples was selected. This corresponds to an update rate of $0.25 \mathrm{~Hz}$.

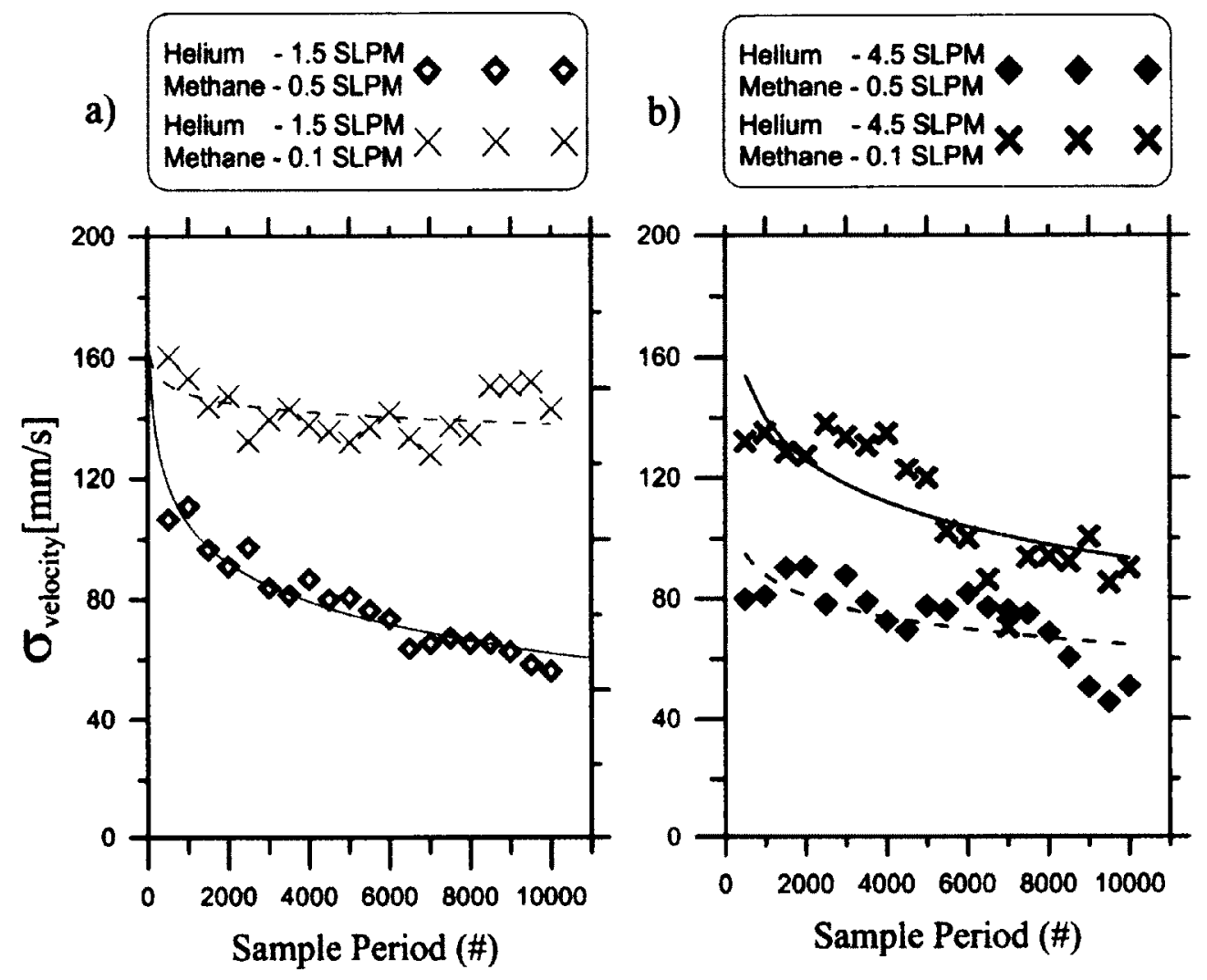

Figure 5.11: Standard deviation of the measured velocity vs. the CCV sample period for Helium- $\mathrm{CH}_{4}$ flows at the (a) minimum and (b) maximum of experimental helium flow range

\subsubsection{Bi-modal flow behaviour}

From the Schlieren images and the CCV data, it was discovered that the buoyant plume had two main modes of fluctuation behaviour. The first was the plume eddy (vertical) mode in which eddies moved along the plume axis; the second was the "flapping" (radial) mode where the plume body itself moved bi-directionally, perpendicular to the main (vertical) flow direction. The radial mode is associated with change in the plume diameter (corresponding to a change in the plume-laser path length), which is attributed both to the effects of entrainment (of the surrounding air) and to minor cross flow disturbances. These effects cause the plume to sway, resulting in a slower "secondary" time delay in the concentration measurements. While the secondary mode generally has 
a lower cross-correlation value (i.e. lower value of $R_{x y}$ ), post-processing was required to avoid incorrect time delay measurements at the lower flow rates. This was done by extracting the measured data corresponding to the first mode, which was made apparent from histogram plots of all the measured time delays for a given test set point, as seen in Figure 5.12.

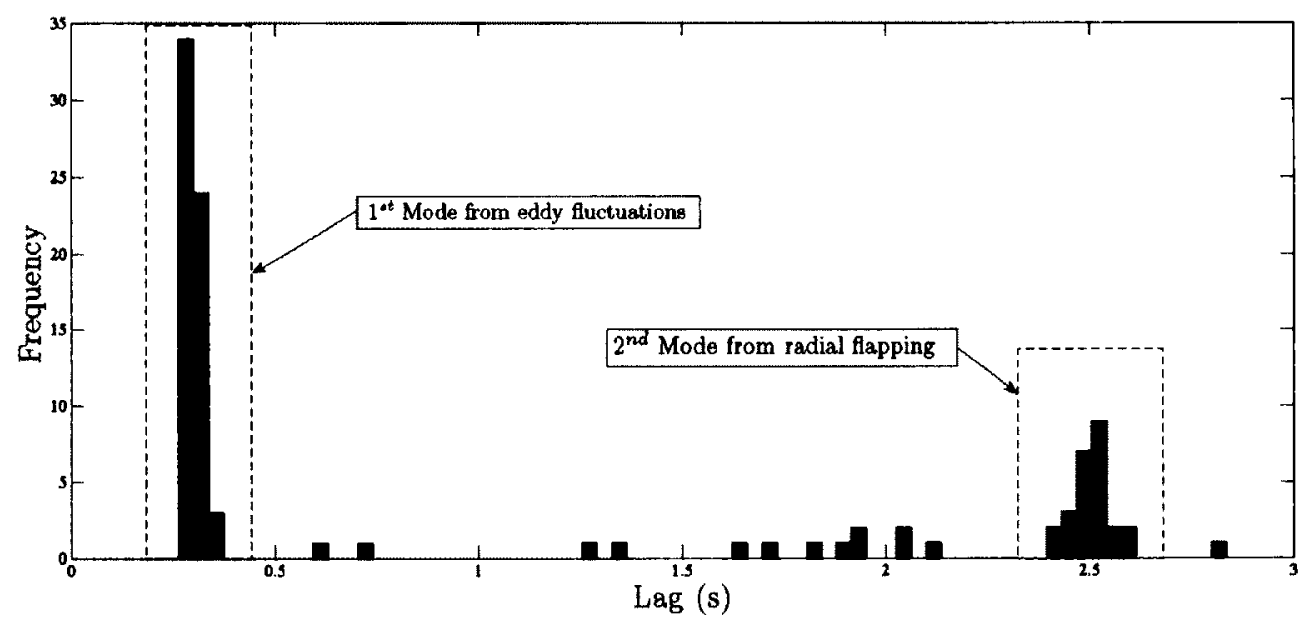

Figure 5.12: Example of a histogram of $\mathrm{CCV}$ extracted time delay for a test point.

Figure 5.13 shows the cross correlation parameter $R_{x y}$ distribution as a function of time lag. The plot shows two main peaks, the dominant one, maximized at $\sim 0.29$ seconds and the minor one maximized at $\sim 2.90$ seconds.

a)

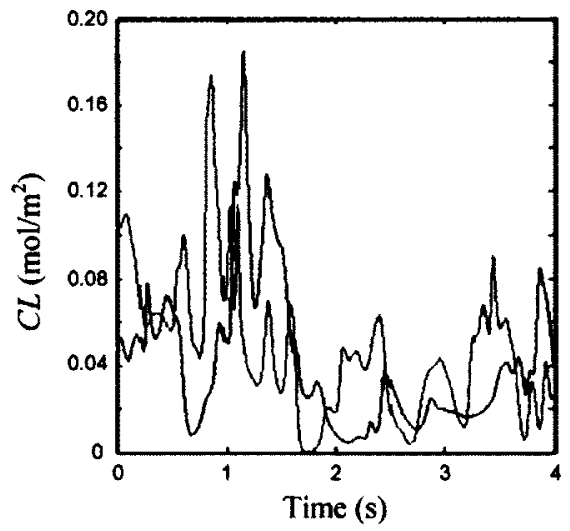

b)

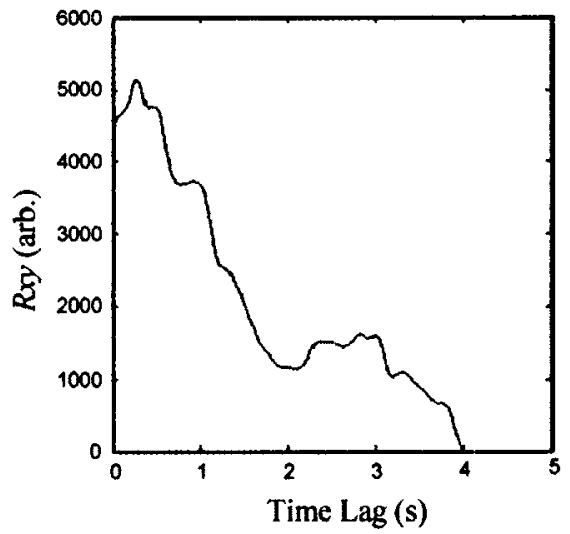

Figure 5.13: (Left) the path integrated concentration of the (red) lower and (blue) upper detectors and the (Right) $\boldsymbol{R}_{x y}$ time lag distribution for the corresponding 4000 samples for a Helium-Methane (1.5/0.1 SLPM) test run 


\subsubsection{Eddy velocity measurements}

From the $\mathrm{CCV}$ time delay measurements, the characteristic velocity of the observed eddies was calculated using Equation 4.19, seen below. The 95\% uncertainty of the time delay $\left(U_{\Delta t_{C C V}}\right)$ was determined using a sample set of 100 and with a student distribution tvalue of 1.985 (95\% confidence level) as outlined in Eq. 4.5. The total precision uncertainty of the measured velocity was calculated using the ASME TSM method.

$$
\begin{gathered}
V_{C C V}=\frac{D_{\text {beam }}}{\Delta t_{C C V}} \\
U_{V_{C C V}}=\sqrt{\left(\frac{1}{\Delta t_{c C V}}\right)^{2}\left(U_{D_{\text {beam }}}\right)^{2}+\left(\frac{D_{\text {beam }}}{\Delta t_{c c V^{2}}}\right)^{2}\left(U_{\Delta t_{C C V}}\right)^{2}}
\end{gathered}
$$

Figure 5.14 and Figure 5.15 shows the measured eddy velocities and their corresponding uncertainties calculated by Eq. 4.20. The air- $\mathrm{CH}_{4}$ mixture depicts the expected linear trend as the methane flow is increased. This is due to the fact that the eddies in the flow are driven by buoyant (and viscous) forces, which scale linearly to the volumetric fraction of the methane since the diluting flow of air has no relative buoyant properties when compared to the ambient surroundings (air). The helium- $\mathrm{CH}_{4}$ eddy velocities on the other hand scale proportionally to the volumetric flow of both the helium and methane flow rates as seen in Figure 5.16. Additionally, the high diffusivity of helium leads to greater variation in the captured velocity as further discussed in Section 5.4.1. 


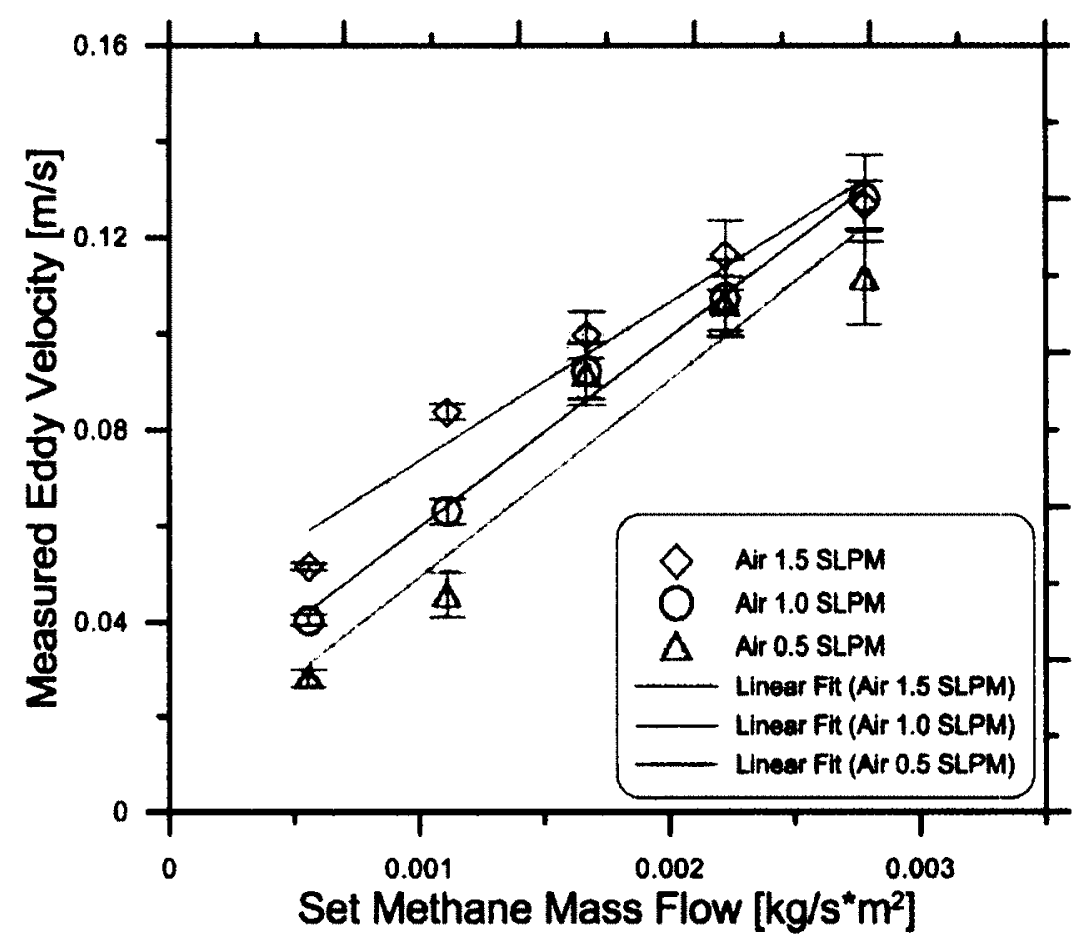

Figure 5.14: Measured eddy velocities of the Air- $\mathrm{CH}_{4}$ plume as a function of methane mass flux

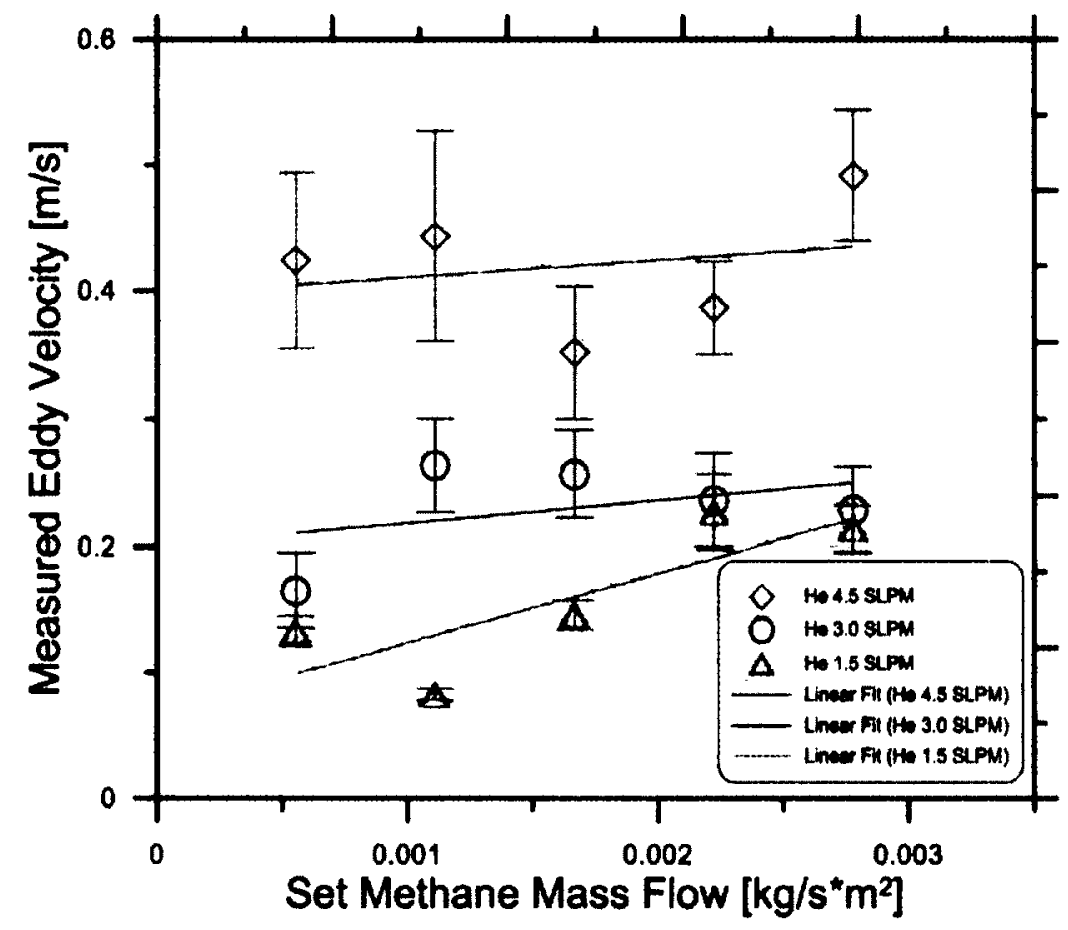

Figure 5.15: Measured eddy velocities for the $\mathrm{Helium}-\mathrm{CH}_{4}$ plume as a function of the methane mass flux 
5.4.1), which increase linearly with the methane flow rate (air- $\mathrm{CH}_{4}$ data) and the dilution flow rate (mainly for the helium- $\mathrm{CH}_{4}$ data).

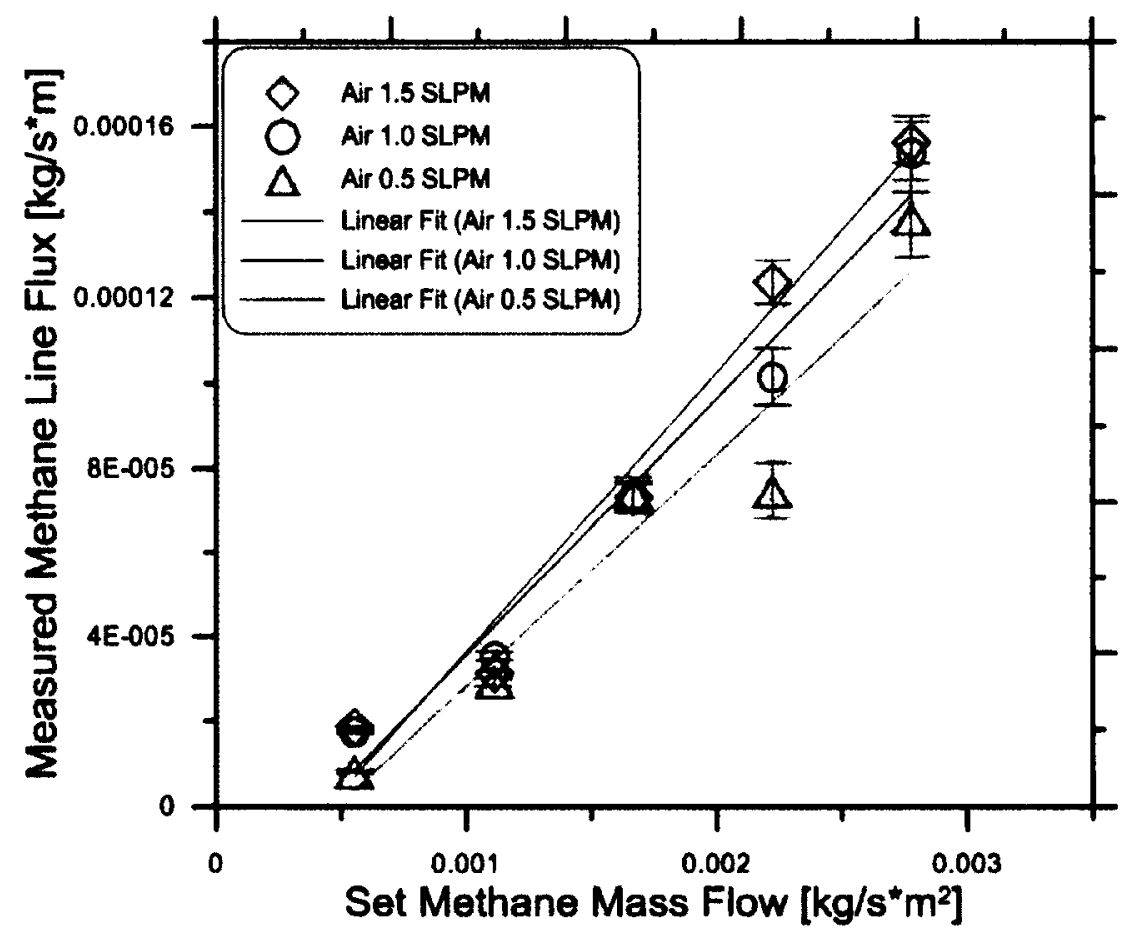

Figure 5.17: Measured Line Flux of Methane for the Air- $\mathrm{CH}_{4}$ mixtures.

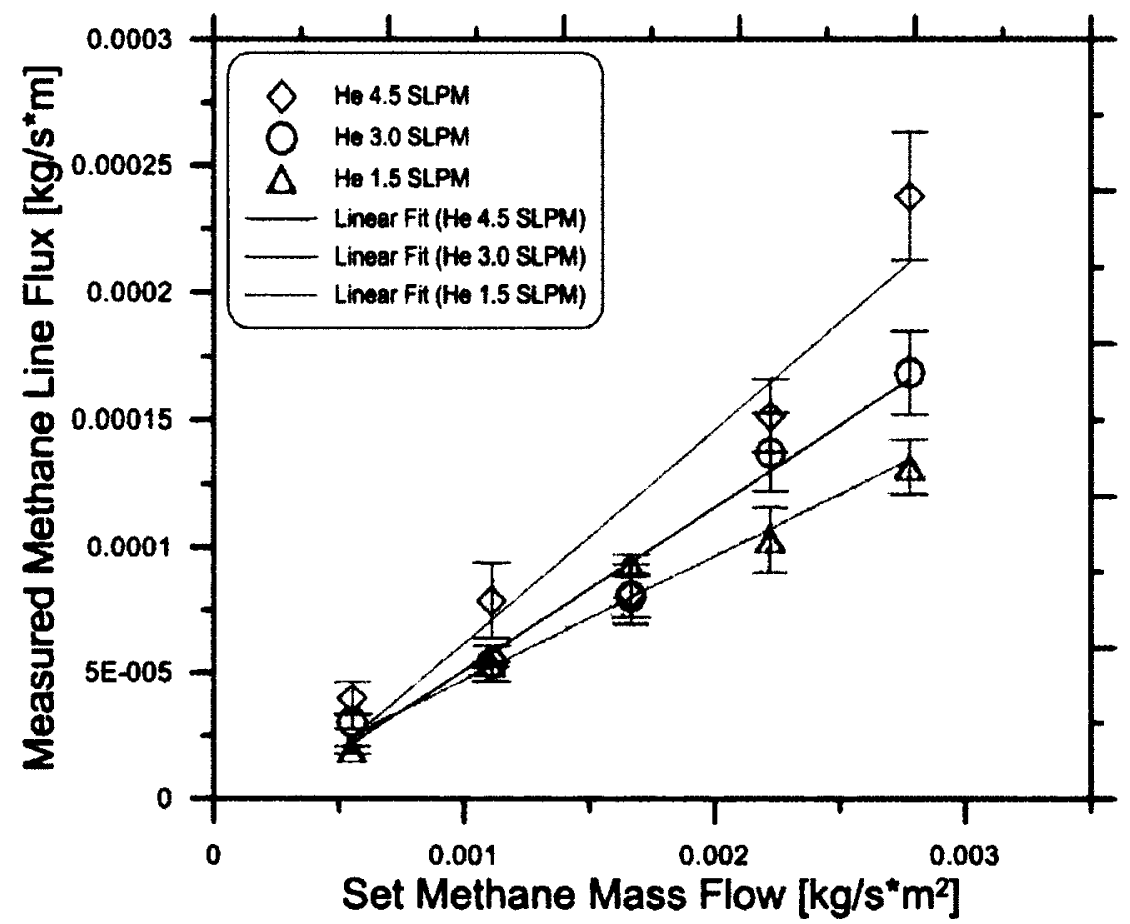

Figure 5.18: Measured Line Flux of Methane for the Helium- $\mathrm{CH}_{4}$ mixtures. 
5.4.1), which increase linearly with the methane flow rate (air- $\mathrm{CH}_{4}$ data) and the dilution flow rate (mainly for the helium- $\mathrm{CH}_{4}$ data).

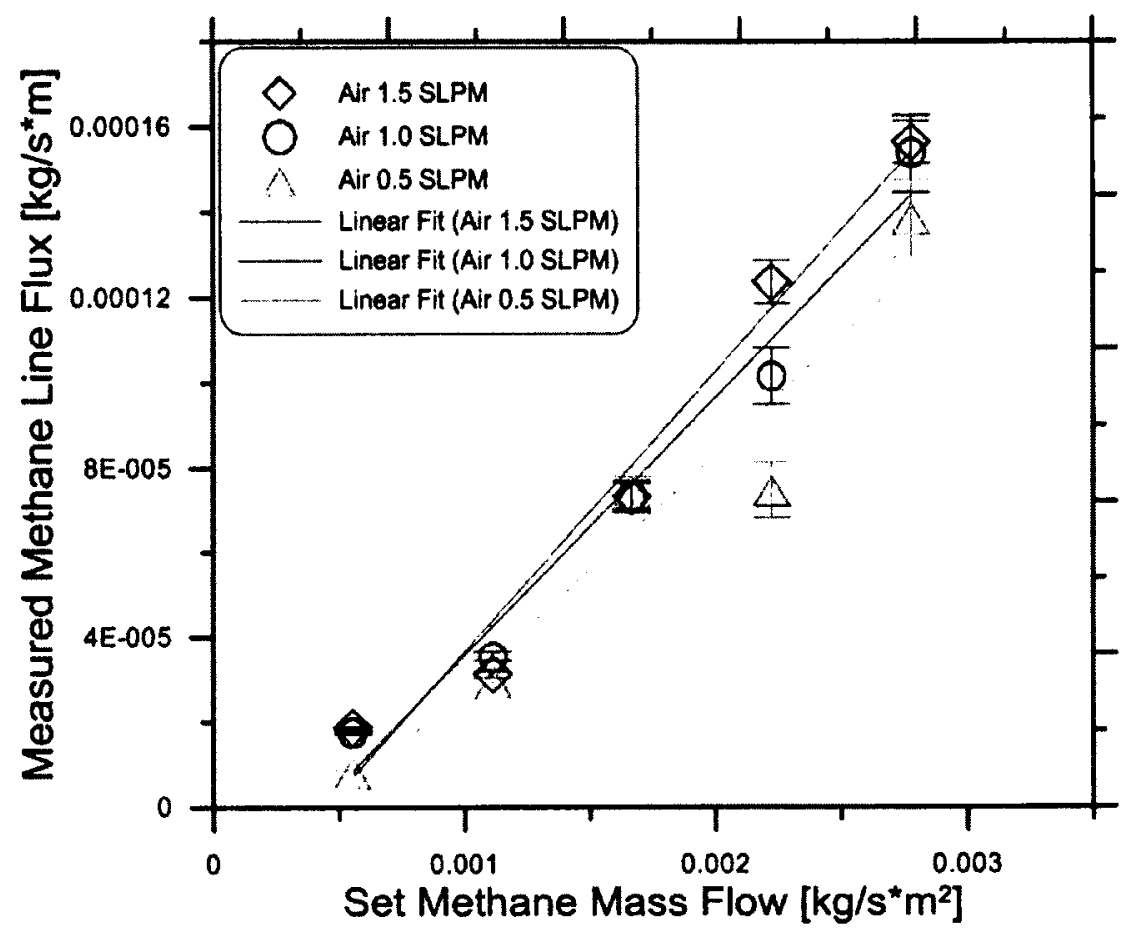

Figure 5.17: Measured Line Flux of Methane for the Air- $\mathrm{CH}_{4}$ mixtures.

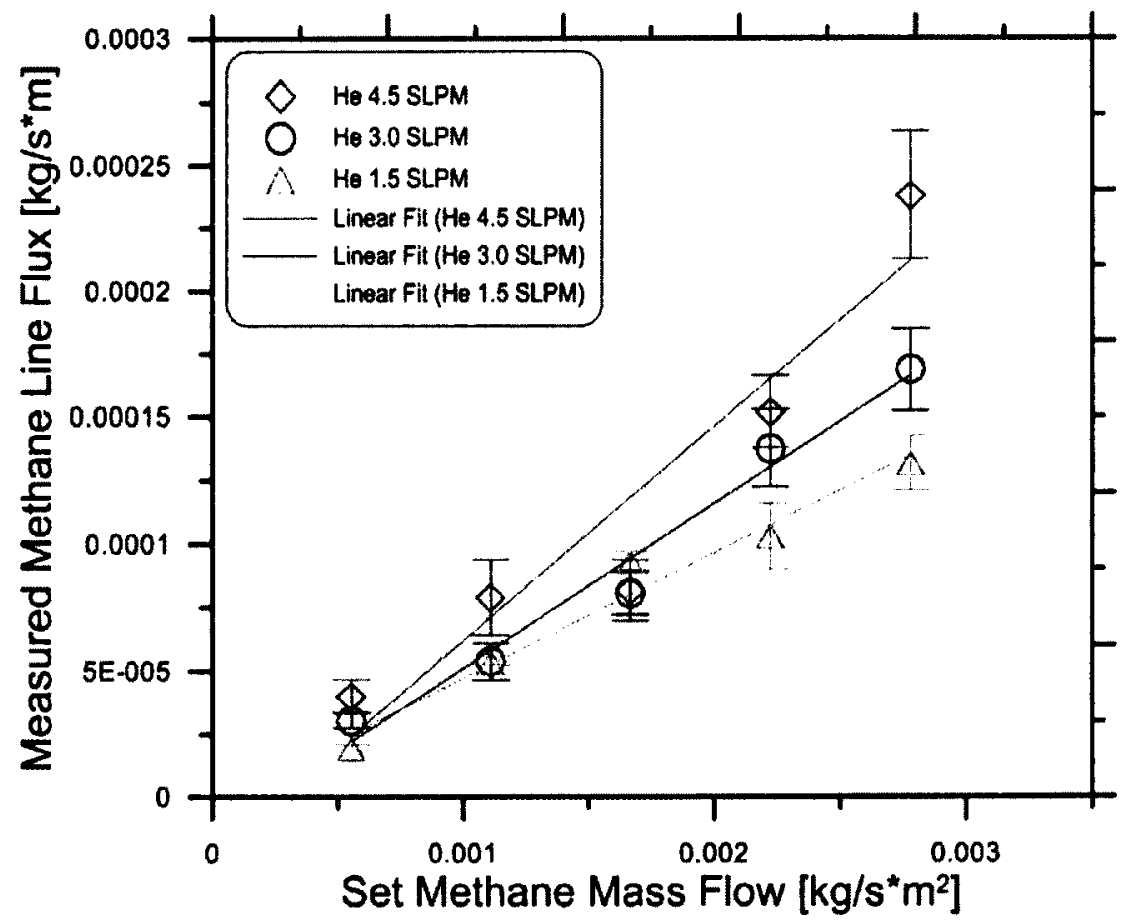

Figure 5.18: Measured Line Flux of Methane for the Helium- $\mathrm{CH}_{4}$ mixtures. 
Table 5.6: The relative uncertainties of the measured line flux of $\mathrm{CH}_{4}$

\begin{tabular}{|c|c|c|c|c|c|c|}
\hline $\begin{array}{c}\text { Methane } \\
\text { Flow rate } \\
\text { (SLPM) }\end{array}$ & \multicolumn{2}{|c|}{ Air Dilution Flow rate } & \multicolumn{3}{c|}{ Helium Dilution Flow rate } \\
\cline { 2 - 7 } & $\mathbf{0 . 5}$ SLPM & $\begin{array}{c}\mathbf{1 . 0} \\
\text { SLPM }\end{array}$ & $\begin{array}{c}\mathbf{1 . 5} \\
\text { SLPM }\end{array}$ & $\begin{array}{c}\mathbf{1 . 5} \\
\text { SLPM }\end{array}$ & $\mathbf{3 . 0}$ SLPM & $\begin{array}{c}\mathbf{4 . 5} \\
\text { SLPM }\end{array}$ \\
\hline 0.1 & $2.68 \%$ & $1.86 \%$ & $3.78 \%$ & $7.21 \%$ & $9.59 \%$ & $16.36 \%$ \\
\hline 0.2 & $2.46 \%$ & $2.88 \%$ & $5.79 \%$ & $1.75 \%$ & $13.42 \%$ & $18.73 \%$ \\
\hline 0.3 & $4.44 \%$ & $5.16 \%$ & $6.02 \%$ & $4.69 \%$ & $10.61 \%$ & $14.71 \%$ \\
\hline 0.4 & $6.86 \%$ & $6.51 \%$ & $3.89 \%$ & $12.55 \%$ & $11.06 \%$ & $9.42 \%$ \\
\hline 0.5 & $3.56 \%$ & $5.87 \%$ & $6.58 \%$ & $8.21 \%$ & $9.73 \%$ & $10.57 \%$ \\
\hline
\end{tabular}

\subsubsection{Mass flow measurements from traverse tests}

A single air- $\mathrm{CH}_{4}$ flow rate was tested to validate the DAS-CCV technique and assess the viability of a multiline grid DAS-CCV sensor. The experiment was conducted using a plume with $1.5 \mathrm{SLPM}$ of air and $0.2 \mathrm{SLPM}$ of $\mathrm{CH}_{4}$. The spatially resolved measurements of the path averaged concentrations of the air- $\mathrm{CH}_{4}$ plume can be seen in Figure 5.19. Measurements were taken at twenty-four traverse positions with $1 \mathrm{~cm}$ spacing, and for each traverse point 60,000 samples were recorded (@1000 Hz). The concentration measurements taken at different traverse positions show the expected parabolic trend over the width of the plume. The plume measurements showed an increased path length in the top laser line (plume) measurements. The traverse test also demonstrated that the concentration measurements were not conducted at the maximum of the concentration profile, possibly resulting in the inconsistency between the path integrated concentration measurements of different dilution flow rates. 


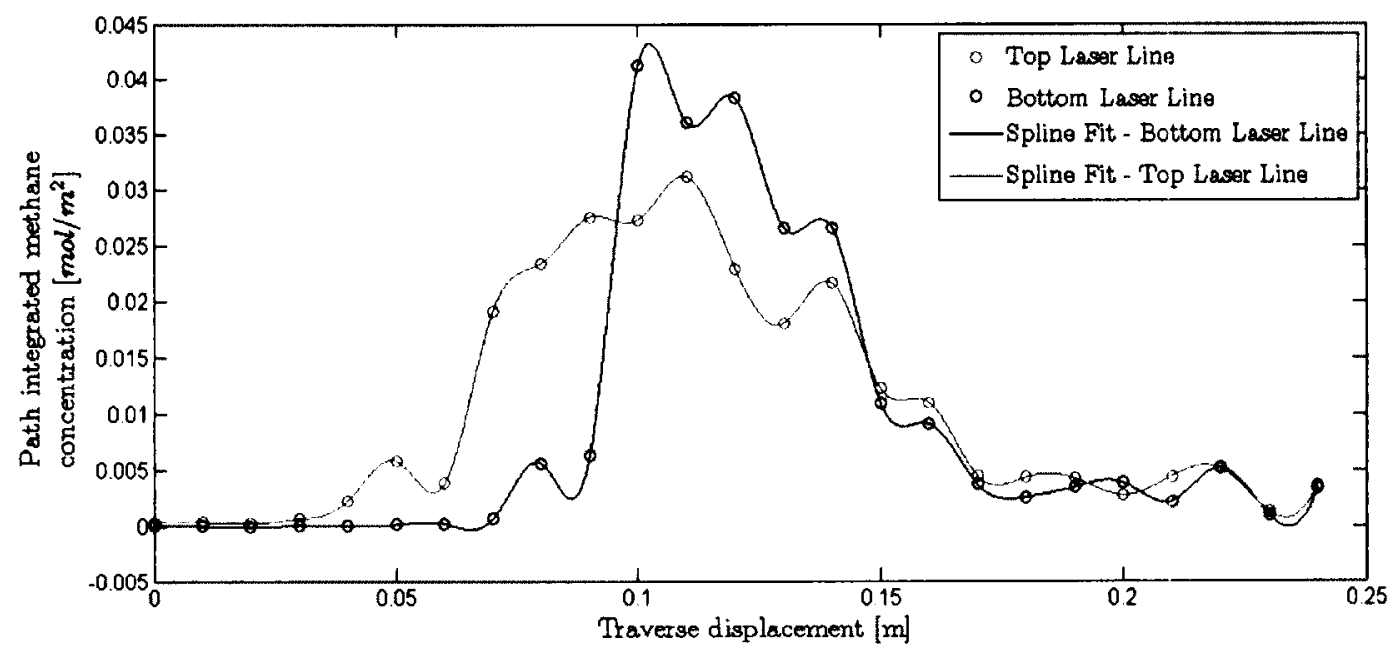

Figure 5.19: Path integrated concentration measurements across the traverse path length

Figure 5.20 shows the eddy velocity measured using DAS-CCV technique at different traverse positions. The distribution of the velocity profile of the plume was found to slightly deviate away from the expected Gaussian profile of an open plume jet, however, the general behaviour and magnitude range falls within the expected values. Using both the path integrated concentration and the velocity distribution, the total $\mathrm{CH}_{4}$ mass flow was calculated by Eq. 4.23. These results are tabulated in Table 5.7 for both the upper and lower plane across the hydrocarbon plume. The measured mass flow measurements showed good agreement to the set mass flow and in the author's opinion validates the progression onto the multiline DAS-CCV phase of experimentation. 


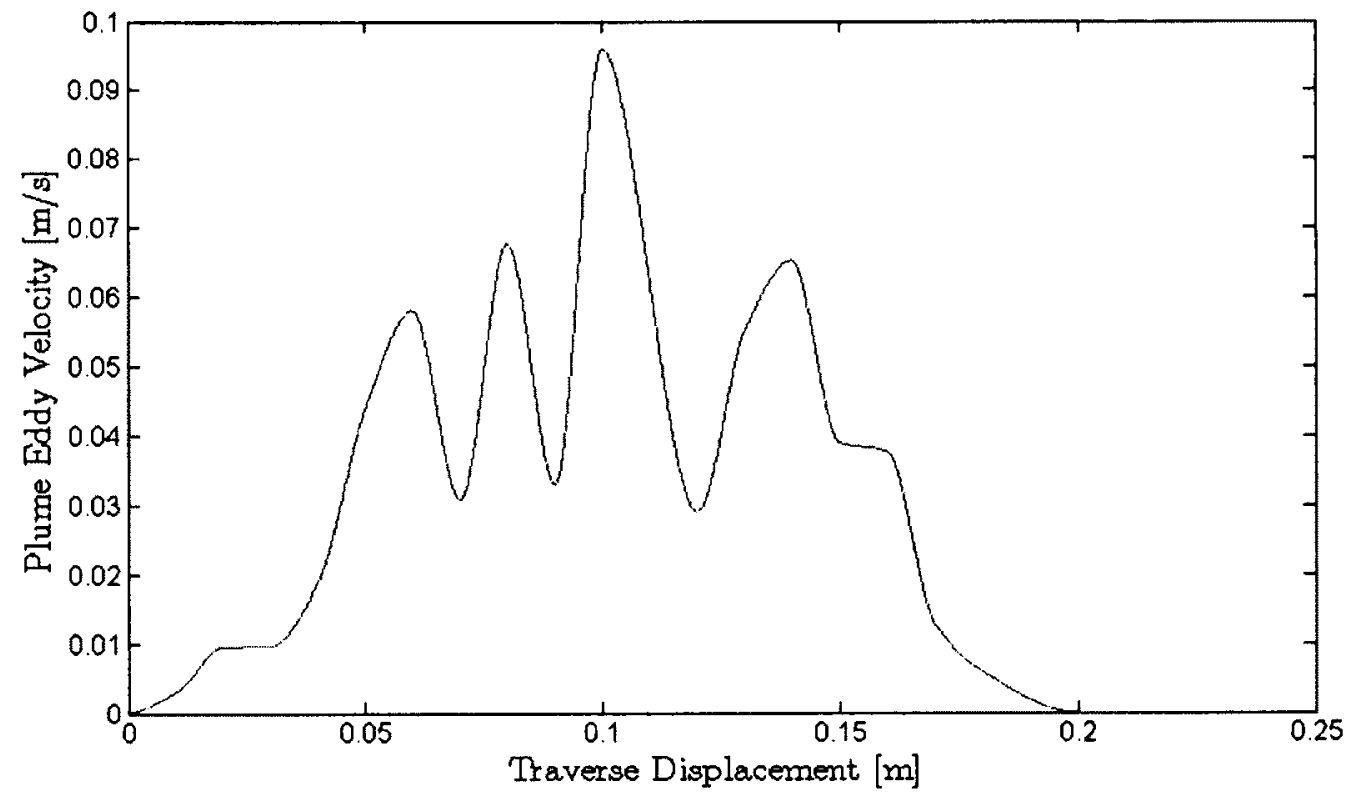

Figure 5.20: Plume eddy velocity measurements across the traverse path length

Table 5.7: Methane mass flow measurements using a traverse

\begin{tabular}{|c|c|c|c|}
\hline Location & $\begin{array}{c}\text { Measured Mass } \\
\text { Flow }[\mathrm{kg} / \mathbf{s}]\end{array}$ & $\begin{array}{c}\text { Set Mass Flow } \\
{[\mathrm{kg} / \mathbf{s}]}\end{array}$ & Error (\%) \\
\hline Top plane & $2.0724 \mathrm{e}-006$ & \multirow{2}{*}{$2.167 \mathrm{e}-006$} & 8.05 \\
\cline { 1 - 2 } Bottom plane & $1.9925 \mathrm{e}-006$ & & 4.37 \\
\hline
\end{tabular}

\subsection{Schlieren Imaging}

The Schlieren imaging apparatus was implemented for two main reasons; to ascertain qualitative information of the plume and to measure the approximate laser-plume path length.

\subsubsection{Buoyant eddy behaviour}

To visualize the plume's behaviour, the Schlieren images were scrutinized with low thresholds to produce images that portrayed the boundaries of the lower density (Methane/Helium) gas. However, since low thresholds were used, a significant amount of the noise produced by the high gain of the acquisition camera remained after the binary 
threshold was implemented. To rectify this, a Gaussian low pass filter was implemented to clean up the images, however, some noise artefacts still remained. The plume's eddies and denser regions (and noisy regions) were highlighted using MatLab's image boundary function to ascertain qualitative information of the plume's behaviour.

From the plots shown below, it was determined that eddy length scales decreased as the dilution flow rate increased, especially with the helium- $\mathrm{CH}_{4}$ mixtures, where the flow range was three times that of the air- $\mathrm{CH}_{4}$ set flow rates. From Rockwell's (2009) experiments using $\mathrm{CCV}$ (with thermocouples), it was determined that the optimal beam spacing satisfied the "frozen eddy" condition. The frozen eddy condition is satisfied when the measured eddy's size and shape remains unaltered as the eddy progresses pass both measurement devices. If the (thermocouples) sensors were placed too close to each other, small errors in time delay measurements yielded great errors in velocity, and the "fluctuations" measured by the downstream sensor would be affected by the wake of the upstream sensor. Conversely, large sensor separation yielded error due to eddy dissipation or generation.

Figure 5.21 and Figure 5.22 illustrate that the eddy length scales for the air- $\mathrm{CH}_{4}$ mixtures are sizable compared to the beam spacing (highlighted in red), which was demonstrated by the relatively narrow $95 \%$ confidence limits of the air- $\mathrm{CH}_{4}$ measured velocities. However, as evident in Figure 5.23 and Figure 5.24, the helium- $\mathrm{CH}_{4}$ eddy length scales were found to be approximately half of the beam spacing. In addition, the helium- $\mathrm{CH}_{4}$ eddies were observed to change as they passed through the laser lines. Figure 5.23 shows two small eddies combining to form another eddy, while Figure 5.24 shows an eddy dissipating as it traverses the two laser beams. The transient eddy behaviour and its length scales are undoubtedly the reason for the large variation of velocities (i.e. error) in the helium- $\mathrm{CH}_{4}$ measured velocities where the beam spacing (per "eddy length") was too large to satisfy the "frozen eddy" condition.

Unlike thermocouple CCV data presented by Rockwell (2009), the nature of DAS is non-intrusive (i.e. the laser does not affect the flow on the macroscopic level), and it stands to reason that the accuracy of the velocity measurement can be improved by lowering the spacing of the two vertically stacked laser beams ensuring that a traversing 
eddy satisfies the "frozen" eddy condition. It should be noted that this claim assumes an ample (A/D) sampling rate to ensure enough transient resolution by which to crosscorrelate the small time delays, and sufficient diversity of the two signals by which a maxima can be found within the $R_{x y}$ parameter.

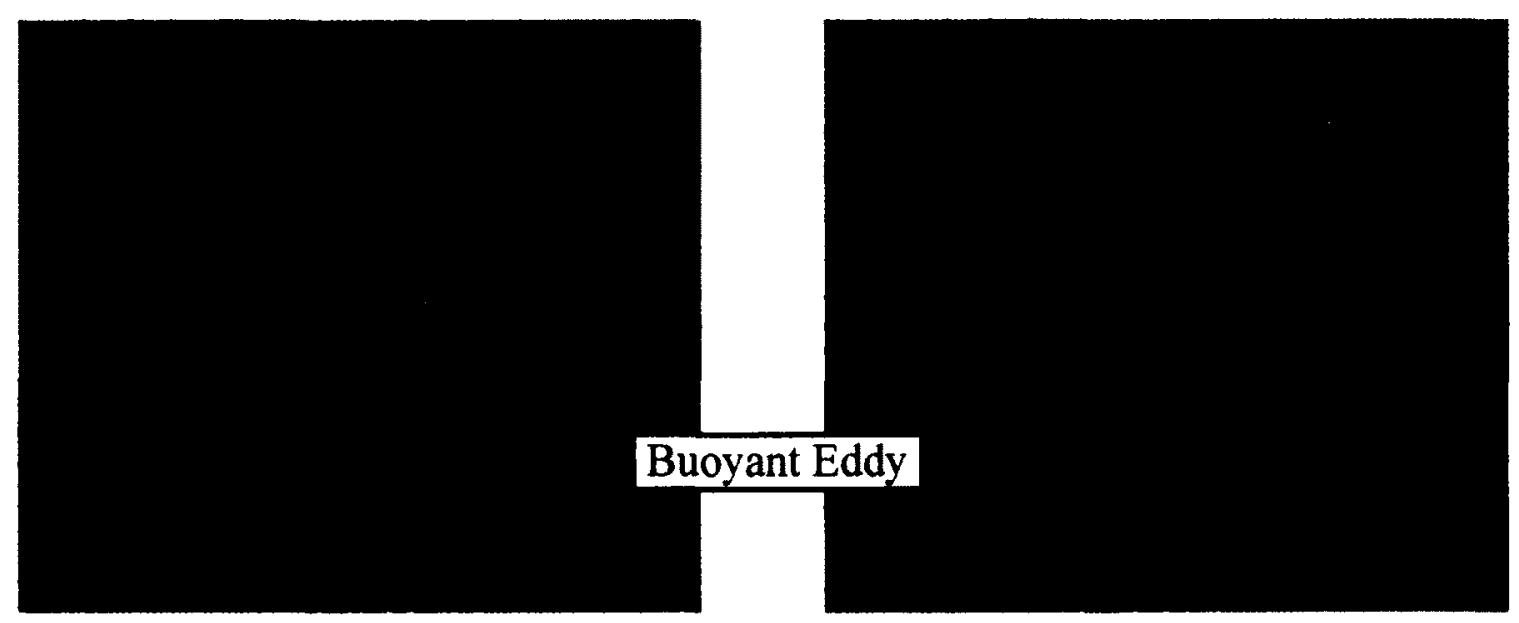

Figure 5.21: Time lapsed images ( $\Delta \mathrm{t}=0.1 \mathrm{~s})$ of air (at $0.5 \mathrm{SLPM}$ ) and $\mathrm{CH}_{4}$ (at 0.5 SLPM)

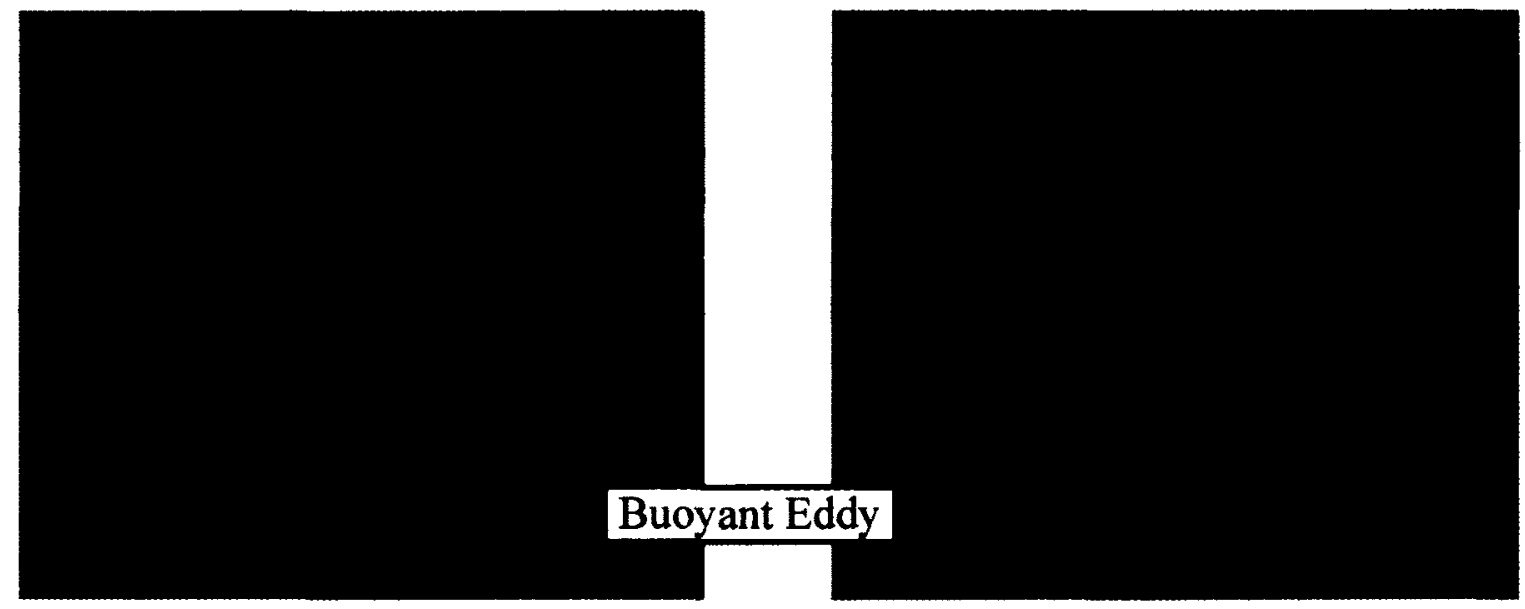

Figure 5.22: Time lapsed images ( $\Delta t=0.1 \mathrm{~s})$ of air (at 1.5 SLPM) and $\mathrm{CH}_{4}$ (at 0.5 SLPM) 


\section{Buoyant Eddy}

Figure 5.23: Time lapsed images ( $\Delta \mathrm{t}=0.1 \mathrm{~s})$ of helium (at 1.5 SLPM) and $\mathrm{CH}_{4}$ (at 0.5 SLPM)

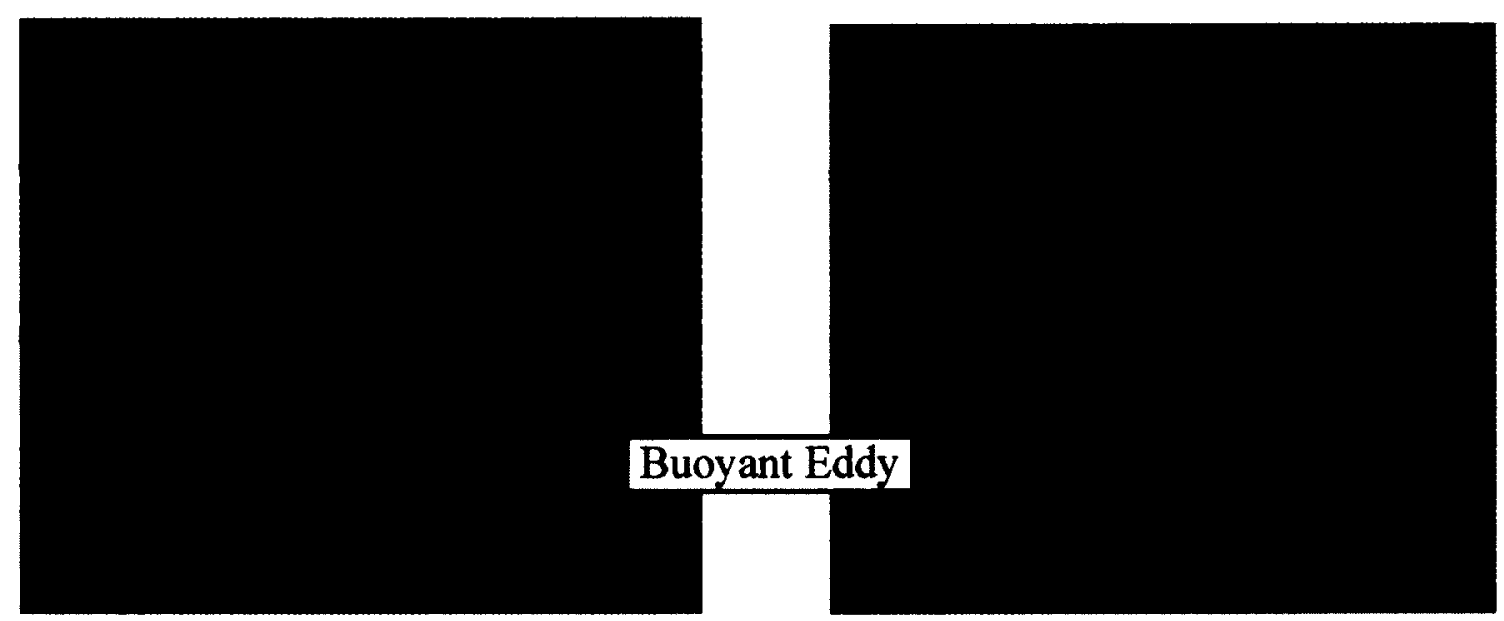

Figure 5.24 : Time lapsed images $(\Delta t=0.1 \mathrm{~s})$ of helium (at 4.5 SLPM) and $\mathrm{CH}_{4}$ (at 0.5 SLPM)

\subsubsection{Plume path length}

To validate the VOC sensor's performance, the approximate plume path length was extracted from the Schlieren images for each test case, yielding qualitative information on the plume behaviour and its influence on the line flux measurements presented in Section 5.3.3. To achieve this task, samples of 100 Schlieren images were scrutinized 
with high (binary) thresholds and averaged to yield a contrast image of the plume body. The plume path length was measured from the resultant image by locating the intensity edges corresponding to the plume diameter. Figure 5.25 shows a plot of the pixel row $(y=155 \mathrm{px}$ from image origin) that corresponds to the approximate location of the average plume-laser path length located between the two laser beams. The average pixel path length was converted into real-world coordinates by utilizing the pixel-world calibration factor $(0.35 \mathrm{~mm} / \mathrm{pixel})$. The average plume path lengths for each test mixtures are tabulated in Table 5.8 in metres.

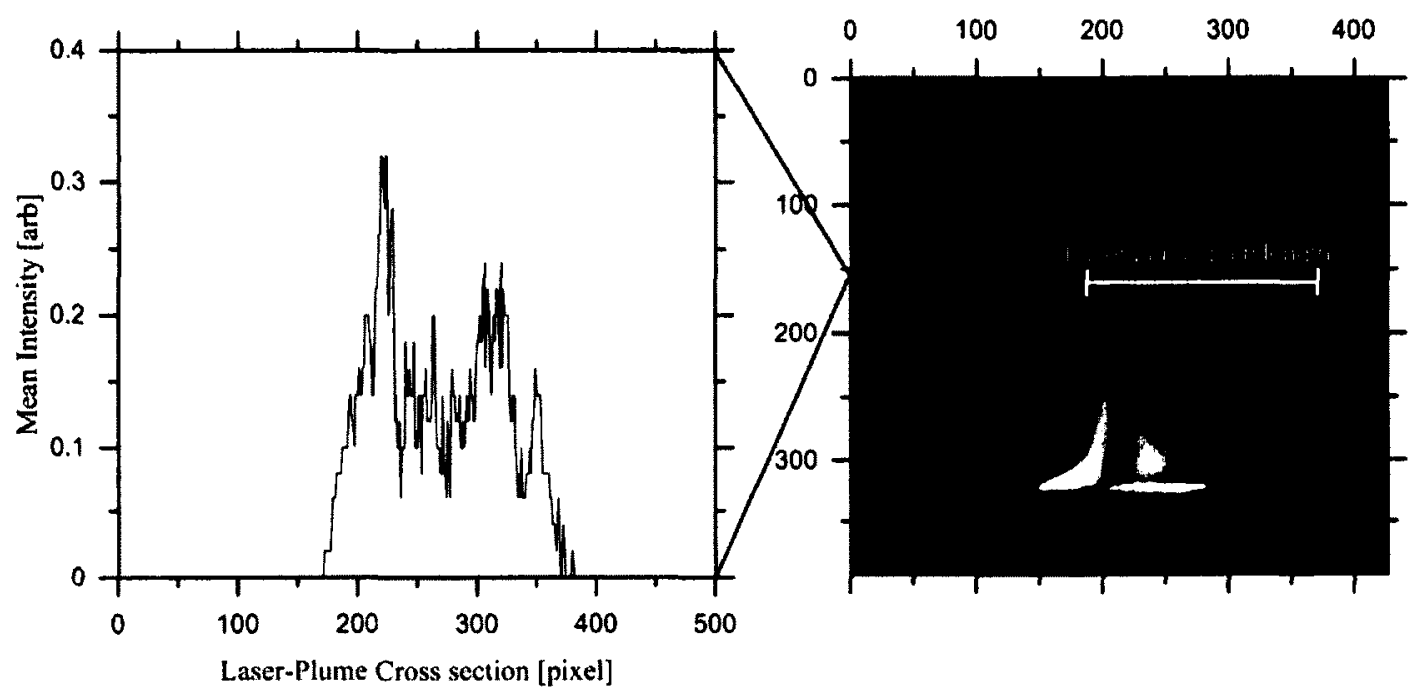

Figure 5.25: Plume-Laser path length measurement of helium (at 4.5 SLPM) and $\mathrm{CH}_{4}$ (at 0.5 SLPM); (Left) the intensity distribution across the laser beam spacing mid-point ( $y=155, x=0$ - 424); (Right) Average of a 100 post-processed Schlieren images

Table 5.8: The average plume path length measured from the post-processed Schlieren images (Units are in metres)

\begin{tabular}{|c|c|c|c|c|c|c|}
\hline $\begin{array}{c}\text { Methane } \\
\text { Flow rate } \\
\text { (SLPM) }\end{array}$ & \multicolumn{2}{|c|}{ Air Dilution Flow rate } & \multicolumn{3}{c|}{ Helium Dilution Flow rate } \\
\cline { 2 - 7 } & $\mathbf{0 . 5}$ SLPM & $\begin{array}{c}\mathbf{1 . 0} \\
\text { SLPM }\end{array}$ & $\begin{array}{c}\mathbf{1 . 5} \\
\text { SLPM }\end{array}$ & $\begin{array}{c}\mathbf{1 . 5} \\
\text { SLPM }\end{array}$ & $\mathbf{3 . 0}$ SLPM & $\begin{array}{c}\text { 4.5 } \\
\text { SLPM }\end{array}$ \\
\hline 0.1 & 0.0220 & 0.0227 & 0.0275 & 0.0417 & 0.0555 & 0.0685 \\
\hline 0.2 & 0.0273 & 0.0287 & 0.0294 & 0.0424 & 0.0599 & 0.0699 \\
\hline 0.3 & 0.0370 & 0.0406 & 0.0367 & 0.0450 & 0.0542 & 0.0692 \\
\hline 0.4 & 0.0427 & 0.0427 & 0.0452 & 0.0431 & 0.0597 & 0.0713 \\
\hline 0.5 & 0.0470 & 0.0530 & 0.0546 & 0.0448 & 0.0613 & 0.0747 \\
\hline
\end{tabular}


For the air- $\mathrm{CH}_{4}$ line flux measurements, the path length was found to increase by a factor $\sim 2$, for an increase of methane from 0.1 SLPM to 0.5 SLPM, which in turn causes the intercept of the fitted lines (as $x \rightarrow 0$ ) to deviate from the expected $y=a x$ trend. However, the lower diffusivity of air (in air) yielded minimal path length change as the air flow rate increased from 0.5 SLPM to 1.5 SLPM, resulting in minimal change in the overall slope between the line flux curves for the different air flow rates, as seen in Figure 5.17. The helium- $\mathrm{CH}_{4}$ data, was found to behave in the opposite fashion, where the path length increased slightly for an increase in the methane flow rate, but significantly increased for increases in the helium flow rate (attributable to the high diffusivity of helium in air, $\sim 4.5$ times higher than that of methane in air) and the higher helium flow rates. This resulted in minimal deviation in the lower tail of the curve (from the expected $y=a x$ trend) but a more pronounced increase in the overall slope of the curve for the different helium- $\mathrm{CH}_{4}$ flux curves. Despite these complications, the proof of concept dual-line CCV experiments demonstrate the potential utility of this approach and issues of varying path-length through the plume should be readily resolved using a multiline, gridded laser beam system capable of resolving the full plume cross-section.

\subsection{Cost-effective critique}

To quantify the cost benefit of the VOC sensor, the total monetary investment was scrutinized under the dollar per laser line criteria. The dollar per laser line is primary controlled by the detector and its associated Mid-IR optical components, both on the laser and detector side. This is due to the ample available power of the HeNe laser source which allows the creation of multiple laser lines per source. Taking this into consideration along with the fact that the future VOC sensor's acquisition electronics would likely be implemented on the microcontroller level, the expected dollar per laser line of the novel VOC sensor is $\sim \$ 2300$, yielding a monetary investment of $\$ 73,600$ for a 32 line VOC sensor. A complete breakdown of the experimental apparatus and its associated costs are shown in Table 5.9. 
Table 5.9: Detailed Cost Breakdown of the Developed Sensor System

\begin{tabular}{|c|c|c|c|c|}
\hline Item No. & Description & Price (\$) & Qty & $\begin{array}{c}\text { Price }{ }^{\star} \text { Qty } \\
\text { (S) }\end{array}$ \\
\hline CP06 & Cage Plate with Double Bore & 16.75 & 3 & 50.25 \\
\hline $\begin{array}{c}\text { CM1- } \\
\text { BP145B } \\
4\end{array}$ & $\begin{array}{l}\text { Cube-Mounted Pellicle Beamsplitter, 45:55, 3- } \\
\text { 5um }\end{array}$ & 250.00 & 2 & 500.00 \\
\hline $\begin{array}{l}\text { CM1- } \\
\text { M01 }\end{array}$ & Cube-Mounted Protected Gold Turning Mirror & 146.00 & 3 & 438.00 \\
\hline $\mathrm{CM} 1-\mathrm{CC}$ & Cage Cube Connector for CM1-4E Series & 45.00 & 1 & 45.00 \\
\hline ER2 & Cage Assembly Rod, 2"Long, $\emptyset 6 \mathrm{~mm}$ & 5.90 & 4 & 23.60 \\
\hline ER05 & Cage Assembly Rod, $1 / 2$ " Long, $06 \mathrm{~mm}$ & 5.00 & 4 & 20.00 \\
\hline ER3 & Cage Assembly Rod, 3" Long, $06 \mathrm{~mm}$ & 6.60 & 4 & 26.60 \\
\hline ER4 & Cage Assembly Rod, 4" Long, $\emptyset 6 \mathrm{~mm}$ & 7.10 & 8 & 56.80 \\
\hline RA90 & $\begin{array}{l}\text { Right Angle Post Clamp, Fixed } 90 \text { Degree } \\
\text { Adapter }\end{array}$ & 9.93 & 2 & 19.86 \\
\hline TRI & PG POST, $\sin X 1$ in $8 / 321 / 420$ & 4.74 & 2 & 9.48 \\
\hline TR2 & PG POST, $\operatorname{Sin} X 2$ in $8 / 321 / 420$ & 5.19 & 1 & 5.19 \\
\hline TR3 & $\begin{array}{c}\text { Post 0.499" Dia x 3" 8-32 Stud 1/4-20 Tapped } \\
\text { Hole }\end{array}$ & 5.42 & 1 & 5.42 \\
\hline TR4 & $\begin{array}{c}\text { Post 0.499" Dia x 4" 8-32 Stud 1/4-20 Tapped } \\
\text { Hole }\end{array}$ & 5.87 & 1 & 5.87 \\
\hline TR6 & $\begin{array}{c}\text { Post 0.499" Dia x 6" } 8.32 \text { Stud 1/4-20 Tapped } \\
\text { Hole }\end{array}$ & 6.77 & 1 & 6.77 \\
\hline PH3 & $\begin{array}{l}\text { Post Holder with Spring-Loaded Thumbscrew, } \\
\mathrm{L}=3 \text { " }\end{array}$ & 8.27 & 2 & 16.54 \\
\hline BA2T2 & Adjustable Mounting Base & 19.20 & 4 & 76.80 \\
\hline $\mathrm{CP} 02 \mathrm{~B}$ & Cage Plate Mounting Bracket & 13.70 & 3 & 41.10 \\
\hline $\begin{array}{l}\text { LA5370- } \\
\mathrm{E}\end{array}$ & $\begin{array}{c}\text { CaF2 Plano-Convex Lens, } f=40.0 \mathrm{~mm} \text {, } \\
\text { DIA }=25.4 \mathrm{~mm}, \text { ARC: } 3-5 \mathrm{um}\end{array}$ & 175.00 & 3 & 525.00 \\
\hline ARV1 & Rotatable V-Clamp Cage Mount & 93.90 & 3 & 281.70 \\
\hline MB810 & $\begin{array}{l}\text { Aluminum Breadboard 8" } \times 10^{\prime \prime} \times 1 / 2, " 1 / 4-20 \\
\text { Thread }\end{array}$ & 143.00 & 1 & 143.00 \\
\hline $\begin{array}{l}30545 / 32 \\
882\end{array}$ & HeNe Laser, 3.39um, $2.0 \mathrm{~mW}$, Polarized 500:1 & 3035.00 & 1 & 3035.00 \\
\hline 64353 & Filter ND LWIR 0.30D $25 \mathrm{~mm}$ & 295.00 & 1 & 295.00 \\
\hline $\mathrm{C} 1502$ & Post V-Clamp Mount & 92.00 & 2 & 184.00 \\
\hline $\begin{array}{c}\text { PVI-3.4- } \\
1 \times 1\end{array}$ & $\begin{array}{l}\text { IR Photovoltaic Uncooled Detector with Optical } \\
\text { Immersion }\end{array}$ & 778.00 & 3 & 2332.64 \\
\hline $\begin{array}{l}\text { ON-930- } \\
44004\end{array}$ & O-Series Non-linear Thermistors & 40.00 & 3 & 120.00 \\
\hline & Electronics costs (for the amplifiers) & $\sim 500.00$ & & 500.00 \\
\hline \multicolumn{4}{|c|}{ Total Estimated Price Excl. Tax } & $\$ 8,763.62$ \\
\hline
\end{tabular}




\section{Chapter 6}

\section{Conclusions and Future Work}

\subsection{Conclusions}

This research project was the first investigation of DAS-CCV motivated by an application to develop an economical VOC sensor that could measure hydrocarbon emission rates from LSTs. The sensitivity and the uncertainty of the proposed DAS-CCV technique were evaluated in proof-of-concept experiments to measure a line flux of a hydrocarbon plume under lab conditions.

Sensor performance was evaluated in two main categories: transient response and noise behavior. From 100 percent step input tests it was determined that detector/amplifier had an average response time of $1.3 \mathrm{~ms}$, inferring a cut-off frequency of $770 \mathrm{~Hz}$. Additionally, the step input tests showed very little overshoot $(\sim 0.03 \%)$ in the detector/amplifier response, resulting in very minimal transients (fluctuations) that could cause erroneous cross correlations. The noise behaviour of the referenced signal showed slight degradation from $1 / \mathrm{f}$ noise. However, the overall magnitude was found to be below $0.1 \%$, yielding good signal sensitivity.

The concentration sensitivity and uncertainty of the VOC sensor was determined in experiments to measure the volumetric fraction of various methane-air mixtures in a flow-through a spectroscopic cell. The steady state measurements demonstrated good concentration sensitivities, with random error magnitudes (without averaging) of $1.27 \%$, inferring a detection limit of $2.22 \mathrm{ppm} \cdot \mathrm{m}$. The uncertainty of the concentration measurements determined using the ASTM TSM method, was found to be very good at $\sim 1.35 \%$. 
The line flux sensitivity and uncertainty were determined by experimentally measuring the path integrated concentration and the velocity of the open flow hydrocarbon plume. The velocity was measured using the DAS-CCV technique (to extract the time delay corresponding to the maximum correlation between the measured signals for the two beams). The air-methane data showed good linear agreement between the measured eddy velocity and the methane mass flux of the open flow plume. The velocity measurement uncertainty for the air-methane data was found to lie between 2 to $7.5 \%$ for a sample set of 100 . The path integrated concentration measurements of the airmethane plumes also showed good linear agreement, with uncertainties ranging between 0.4 to $0.6 \%$. Combining the measured eddy velocity and the path integrated concentrations, the calculated air-methane line flux data showed good linear agreement with the input mass flux with total uncertainties ranging between 2.5 to $6.9 \%$.

The helium-methane data allowed tests at higher flow rates while still maintaining sufficient density differences between the plume and the surrounding air for the Schlieren imaging system. However, the measured helium-methane eddy velocities deviated from the input methane mass flow to the plume, as the helium dilution flow increased. This is due to the eddy behaviour being determined by both the methane and helium mass flow, since both gases contribute to the buoyant nature of the plume. For the helium-methane mixtures, the velocity uncertainty was found to lie between 5 to $16.5 \%$ for a sample set of 100. Path integrated concentration measurements of the helium-methane plumes were achieved with uncertainties ranging between 0.4 to $0.55 \%$. From the measured eddy velocity and the path integrated concentrations, the calculated helium-methane line flux depicted good linear agreement with the input flow rate to the plume generator, with higher uncertainties ranging between 2 to $19 \%$. This was attributable to the relative difference between the beam spacing and the characteristic eddy size of the plume, as seen through the Schlieren imaging system, indicating that the beam spacing needed to be reduced to satisfy the frozen eddy condition. Schlieren imaging confirmed this was not a significant issue for the air-methane plumes 
Setting aside these minor issues, from the traverse tests, the dual beam, proof-ofconcept system demonstrated that the DAS-CCV technique is a viable approach that could potentially be utilized in a full scale, multi-beam, gridded sensor to measure the emissions released by LSTs with good accuracy and for a relatively low monetary investment.

\subsection{Future Work}

The four main issues that require further research to move the DAS-CCV sensor to the field are the improvement of the detector-amplifier noise performance, control of the CCV beam spacing, more rigorous consideration of the hydrocarbon speciation problem, and the implementation of the multi-line grid DAS-CCV sensor.

\subsubsection{Detector-Amplifier Noise Performance}

From the noise performance tests, it was determined that the highest noise contributor was either the PVI-3.4 detector or the $1^{\text {st }}$ stage of the custom amplifier (or both). The $1 / \mathrm{f}$ noise, commonly seen with use of semi-conductors, was found to be the highest noise source in the system response. To reduce the $1 / \mathrm{f}$ noise at the detector, the amplifier requires a redesign to allow the detector to be connected with a zero voltage bias (i.e. connected to ground). According to the manufacturer, this will reduce the $1 / \mathrm{f}$ noise produced by the detector. The amplifier requires a detailed analysis and if need be, a redesign to remove any possible $1 / \mathrm{f}$ noise contributors (if any exist).

\subsubsection{Optimal Beam spacing}

As stated previously, the reason for higher error in the velocity measurements of the helium case study was the due to the fact that the beam spacing between the laser-beams was too large, and could not satisfy the "frozen eddy" condition. The beam spacing could thus be better optimized and it would be advisable to design a field system where this might be easily varied using custom-made beam splitting optics. 


\subsubsection{Hydrocarbon speciation}

To move the VOC sensor to field, the hydrocarbon speciation problem must be addressed while maintaining the cost effective design requirements. The hydrocarbon speciation problem lies in the fact that the emissions from LSTs are a multi-component mixture that has high variation in composition, and while these hydrocarbon molecules all have good absorption strengths in the mid-IR band, determining the individual concentration of each gas is impossible from DAS unless the composition was known a priori. Therefore, to make the VOC sensor viable as a fully quantitative tool, further research and design for the speciation problem must be conducted. Likely solutions include secondary extractive gas sampling and analysis (e.g. via gas chromatography or combined gas chromatography mass spectrometry) or secondary near-IR DAS. Extractive gas sampling could potentially be conducted semi-continuously using a field deployable micro-gas chromatograph to determine relative species concentrations and allow for quantitative calibration of the DAS signal. Alternatively, future research could consider the use of one or two near-IR DAS (or WMS) laser lines within the mid-IR multi-line laser grid to use the overtones of the fundamental absorption bands to differentiate between the multicomponent mixtures. A point of interest that will most likely limit (or prevent) the application of a secondary near-IR sensor is the location of all the VOC absorption peaks across the near-IR (wavelength) bandwidth and the strong interference of $\mathrm{H}_{2} \mathrm{O}$ absorption. While the near-IR absorption peaks associated with hydrocarbon are sizably more spread out over the light spectrum (for a singular species) than their mid-IR counterparts, research conducted by Chung (2007) indicated that near-IR absorption of crude oil and gasoline vapours proved difficult due to the overlapping of absorption features corresponding to all of the hydrocarbon species that comprise crude oil and gasoline. For example, gasoline has $\sim 150$ individual species from $C_{4}$ to $C_{9}$ (hydrocarbons) which correspond to several hundred overlapping absorption features, preventing selective measurement of each species. 


\subsubsection{Multi-line CCV}

To meet the ultimate goal of developing a viable means for measuring emissions from external floating roof tanks for which no practical alternatives exist, a critical next step in the development of the proof-of-concept apparatus will be to design and test a costeffective multi-beam system. Related research into tomographic data processing would also be beneficial. 


\section{References}

AIST, N. I. (2010). Spectral Database for Organic Compounds SDBS. Retrieved Febuary 20, 2010, from http://riodb01.ibase.aist.go.jp/sdbs/cgi-bin/direct_frame_top.cgi

Amstrong, I., Johnstone, W., Duffin, K., Lengden, M., Chakraborty, A., \& Ruxton, K. (2010, May 15). Detection of CH4 in the Mid-IR Using Difference Frequency Generation With Tunable Diode Laser Spectroscopy. Journal of Ligthwave Technology, 28(10).

Atcheson, B. (2007). Schlieren-Based Flow Imaging. M.A.Sc Thesis, University of British Columbia, Computer Science.

Bosch Jr., J., \& Logan, T. (2006). VOC Fugitive Losses: New Monitors, Emision Losses, and Potential Policy Gaps. U.S. Environmental Protection Agency.

Chambers, A. K. (2004). Optical Measurement Technology for Fugitive Emissions from Upstream Oil and Gas Facilities. Carbon and Energy Management, Alberta Reseach Council Inc.

Chambers, A., Strosher, M., Wootton, T., Moncrieff, J., \& McCready, P. (2006). DIAL Measurements of Fugitive Emissions from Natural Gas Plants and the Comparison with Emission Factor Estimates. Alberta Research Council.

Cheng, L., Jeffries, J., \& Hanson, R. (2010). Mass Flux Sensing via Tunable Diode Laser Absorption of Water Vapor. AIAA Journal, 48(11).

Chu, T., \& Hogg, D. (1966, Febuary). The Attenuation of 3.392 um He-NE Laser RAdiation by Methane in the Atmosphere. The Bell System Techincal Journal.

Cockburn, J. (2006). Mid-infrared Quantum Cascade Lasers. In Mid-infrared Semiconductor Optoelectronics (Vol. 118). Springer Series in Optical Sciences.

Coleman, H. (2009). Experimentaiton, validation and uncertainty analysis for engineers (3rd Edition ed.). Hoboken, New Jersey: John Wiley \& Sons.

Cox, G., \& Chitty, R. (1980). A Study of the Deterministic Properties of Unbounded Fire Plumes. Combustion and Flame, 39, 191-209.

Daun, K. (2010). Infrared species limited data tomography through Tikhonov reconstruction. Journal of Qualitative Spectroscopy and Radiative Transfer, 111.

Dereniak, E., \& Boreman, G. (1996). Infrared Detectors and Systems. New York: John Wiley \& Sons Inc. 
Engineering Science, I. (1977). Hydrocarbon Emissions from Fixed-Roof Petroleum Tanks.

Env. Can., E. C. (2008). Canada's Greenhouse Gas Emissions: Understanding the Trends. Retrieved September 29, 2009, from http://www.ec.gc.ca/Publications/75805F15-908C-4FB1-A2856D437FF04C93/CanadasGreenhouseGasEmissionsUnderstandingTheTrends 1990 2006.pdf

EPA, E. P. (1995). AP 42: Compilation of Air Pollutant Emission Factors, Volume I: Stationary Point and Area Sources.

Ferry, R. (2002). Emissions from Aboveground Storage Tanks. (TGB partneship) Retrieved Febuary 20, 2011, from http://www.tgbpartnership.com/linked_data_files/tanks_3_0.pdf

Gieseler, H., Kessler, W., Finson, M., Davis, S., Mulhall, P., Bons, V., et al. (2007, July). Evaluation of Tunable Diode Laser Absorption Spectroscopy for In-Process Water Vapor Mass Flux Measurements During Freeze Drying. JOURNAL OF PHARMACEUTICAL SCIENCES, 96(7).

Grant, W. (1986, March 1). He-Ne and cw CO2 laser long-path systems for gas detection. Apllied Optics, 25(5).

Herrmann, K., Renneberg, A., \& Kreuzer, H. (1987). Precision velocimetry with digital cross-correlation for flow measurements during thrombus growth. Microvascular research, 34(1), 13-28.

Hindle, F., Carey, S., Winterbone, D., Clough, E., \& McCann, H. (2001). Measurement of gaseous hydrocarbon distribution by a near-infrared absorption tomography system. Journal of Electronic Imaging, 10(3).

HITRAN, H.-S. C. (2008). The HITRAN 2008 molecular spectroscopic database. Retrieved December 1, 2009, from http://cfa-www.Harvard.edu/HITRAN

Hollas, J. (2004). Modern Spectroscopy. John Wiley \& Sons, Inc.

IMPEL Network. (2000). Diffuse VOC Emisions. Brussels.

Klingbeil, A., Jeffries, J., \& Hanson, R. (2006, June 12). Temperature- and pressuredependent absorption cross sections of gaseous hydrocarbons at $3.39 \mathrm{um}$. Measurement Science and Technology, 1950-1957.

Kllingbeil, A. (2007). MID-IR Laser Absortion Diagnostics for Hydrocarbon Sensing in Harsh Enviroments. Ph.D. Thesis, Stanford University, Department of Mechanical Engineering, Stanford. 
Kosterev, A., Wysocki, G., Bakhirin, Y., So, S., Lewicki, R., Fraser, M., et al. (2008). Application of Quatum Cascade Lasers to Trace Gas Analysis. Applied Physics B, 90, 165-176.

Kuhnemann, F., Muller, F., G.V., B., Halmer, D., Popp, A., Schiller, S., et al. (2004). $\mathrm{CW}$-OPO based cavity-leak-out spectrometer for ultra-sensitive and selective mid infrared trace gas analysis. Proceedings of the SPIE, 5337, 117-127.

Lachish, U., Rotter, S., Adier, E., \& El-Hanany, U. (1987, June). Tunable diode laser based spectroscopic system for ammonia detection in human respiration. Review of Scientific Instuments, 58(6).

Land and Marine Project Engineering Ltd. (n.d.). Internal Floating Roof. Retrieved Febuary 20, 2011, from

http://www.landandmarine.com/TankServProducts/InternalFloatingRoof.aspx

Long, B., \& Garner, B. (2004). Guide to Storage Tanks and Equipment. John Wiley and Sons.

Lubken, F., Dingler, F., Lucke, H., Anders, J., Riedel, W., \& Wolf, H. (1999, September 1). MASERATI: a rocketborne tunable diode laserabsorption spectrometer. Apllied Optics, 38(25).

Lyle, K. (2005). Developement of a Real-Time Diode -Laser Mass flux Sensor for simultaneous Measurement of Density and Velocity of Oxygen. TSD Report, Stanford University, High Temperature Gasdynamics Laboratory, Mechanical Engineering Department, Stanford.

Mell, W., McGrattan, K., \& Baum, H., (1996). Numerical simulation of combustion in fire plumes. 26th Symposium on Combustion/The combustion Institute.

Mellqvist, J., Samuelsson, J., Galle, B., \& Kihlman, M. (2006). The Solar Occultation Flux Method, a new techniue to quantify fugitive VOC emissions. CEM 2006. 7th International Conference on Emission Monitoring". Paros.

Mellqvist, J., Samuelsson, J., Offerle, B., Salberg, H., Johansson, J., \& Jaakkola, S. (2009). Emission Measurements of Volatile Organic Compounds with the SOF method in the Rotterdam Harbor 2008. Goterborg: FluxSense.

Mielenz, K., \& Nefflen, K. (1965). Gas Mixtures and Pressures for Optimum Output Power of Rf-Excited Helium-Neon Gas Lasers at $632.8 \mathrm{~nm}$. Applied Optics, $4(5)$.

Mohamed, A., \& Lefebvre, M. (2009). Laser Absorption Spectroscopy to probe chemically reacting flows. The onera journal Aerospace Lab(1). 
Morgan, M., \& Bowles, K. (1968, September 13). Cross-Correlation and Cross-Spectral Methods for Drift Velocity Measurements. Science, New Series, 161(3846), 11391142 .

Myers, P. (1997). Aboveground Storage Tanks. New York: McGraw-Hill .

Pal, S., Ozanyan, K., \& McCAnn. (2008). A computational study of tomographic measurement of carbon monoxide at minor concentrations. Measurement Science and Technology, 19(9).

Pashotta, R. (2008). Encyclopedia of Laser Physic and Technology, Vols.b I \& II". Weinheim: Wiley-VCH Verlag GmBH \& Co.

Pasley, H., \& Clark, C. (2000). Computational fluid dynamics study of flow around a floating-roof oil storage tanks. Journal of Wind Engineering and Industrial Aerodynamics, 86.

Philippe, L., \& Hanson, R. (1991, December 15). Laser-absorption mass flux sensor for high-speed airflows. Optics Letters, 16(24).

Picard, D. (2009). Estimating Average and Instantaneous Emmssions Rates from Hydrocarbon Liquid Storage Taniks. Clearstone Engineering Ltd.

Piotrowski, J., \& Rogalsk, A. (2007). High Operating Temperature Infrared Photodetectors. Washington: SPIE.

Pouchert, C. (1981). The Aldrich libraryof infrared spectra. Milwaukee: Aldrich Chemical Co.

Rieker, G. (2009). Wavelength-Modulation Spectroscopy for Measurements of Gas Temperature and Concentraiton in Harsh Enviroments. $\mathrm{PhD}$ Thesis, Stanford University, Mechanical Engineering.

Rockwell, S., Rangwala, A., \& Klein, A. (2009). An investigation into the use of Cross Correlation Velocimetry. MSc Thesis, Worcester Polytechnic Institute.

Settles, G.S. (2001). Schlieren and shadowgraph techniques: visualizing phenomena in transparent media. New York: Springer-Verlag.

Shorter, J. H., Nelson, D. D., Milton, D. K., Sama, S., \& Zahniser, M. S. (2010). Clinical Study Of Multiple Biomarkers In The Breath Of Asthma And COPD Patients Using A Fully Automated Quantum Cascade Laser Spectrometer. American Journal of Respiratory andCritical Care Medicine.

Skeldon, K., McMillan, L., Wyse, C., Monk, S., Gibson, G., Patterson, C., et al. (2006). Application of laser spectroscopy for measurement of exhaled ethane in patients with lung cancer. Respiratory Medicine, 100, 300-306. 
So, S., Sani, A., Zhong, L., Tittel, F., \& Wysocki, G. (2009, April 16). Laser Spectroscopic Trace-Gas Sensor Networks for Atmospheric Monitoring Applications. Workshop on Sensor Networks for Earth and Space Science Applications (ESSA).

Spectrasyne Ltd. (2010). Spectrasyne, Enviromental Surveying. Retrieved October 20, 2010

Springholz, G., Schwarzl, T., \& Heiss, W. (2006). Mid-infrared Vertical Cavity Surface Emitting Lasers based on the Lead Salt Compounds. In Mid-infrared Semiconductor Optoelectronics (Vol. 118). Springer Series in Optical Sciences.

Svensson, T., Andersson, M., Rippe, L., Svanberg, S., Andersson-Engels, S., Johansson, J., et al. (2008). VCSEL-based oxygen spectroscopy for structural analysis of pharmaceutical solids. Applied Physics B: Laser and Optics , 345-3554.

Thorlabs Ltd. (2011). Retrieved Febuary 10, 2011, from Available: http://www.thorlabs.com/

Tittel, F. K., Richter, D., \& Fried, A. (2003). Mid-Infrared Laser Applications in Spectroscopy. In I. T. Sorokina, \& K. L. Vodopyanov (Eds.), Solid-State MidInfrared Laser Sources (Vol. 89, pp. 445-510). Springer-Verlag.

Tittel, F., Lancaster, D., Richter, D., Golberg, L., \& Koplow, D. (1999). Compact MidInfrared Trace Gas Sensor Based on Difference Frequency Mixing. SPIE, 3758.

Topfer, T., Petrov, K., Mine, Y., Jundt, D., Curl, R., \& Tittel, F. (1997, October 20). Room-temperature mid-infrared laser sensor for trace gas detection. Applied Optics, 36(30).

Weber, M. (1999). Handbook of Laser Wavelengths. CRC Press LLC.

Wofsy, S. (2011). HIAPER Pole-to-Pole Observations (HIPPO) fine-grained, globalscale measurements of climatically important atmospheric gases and aerosols. Philosophical Transactions of The Royal Soceity A: Mathematical Physical \& Engineering Sciences, 369, 2073-2086.

Wright., P., Garcia-Stewart, C., Carey, S., Hindle, F., Pegrum, S., Colburne, S., et al. (2005, November 1). Toward in-cylinder absorption tomography in a production engine. Applied Optics, 44(31). 


\section{Appendix A}

\section{Mechanisms of Evaporative Losses from Liquid Storage Tanks}

Evaporative losses from liquid storage tanks can be generalized into three main categories: standing losses, working loses, and flashing losses. The relative contributions of the various modes of emissions differ with tank design.

\section{A.1 Working Losses of Liquid Storage Tanks}

Working losses occur during the emptying-filling cycles as the liquid level changes. For fixed roof tanks and horizontal tanks, working losses are comprised of the hydrocarbon saturated air that is displaced when the liquid level rises (i.e. during the filling cycle)(Myers, 1997; EPA, 1995). For EFRTs, working losses mostly are due to "clingage losses", which occur during the withdrawal phase (i.e. the emptying cycle) when the residual organic liquid on the side walls is exposed to the atmosphere allowing for convective evaporation. While clingage does occur in IFRTs, the fixed roof (above the internal floating roof) shields the crude oil clingage from convective mass transfer (due to cross wind flows) only allowing diffusion/evaporative mass transfer. However, EPA (1995) does not distinguish between the two modes of mass transfer and simply states that it is proportional to the exposed surface area and annual throughput (flow rate) (Myers, 1997).

\section{A.2 Standing Losses of Liquid Storage Tanks}

Standing storage losses are directly dependent on the storage tank design due to the fact that they encompass all the operational losses from all the operational fittings and seals of LSTs. Standing emission losses are defined as the evaporative losses that occur independent of liquid-level change in the tank (EPA, 1995). They are the losses that are 
driven by changes in temperature and pressure. For EFRTs, standing losses also include any evaporative losses driven by crosswind flow (Myers, 1997; EPA, 1995). These driving forces result in a differential pressure across the various fittings which promote losses through either the pressure valves or through seal permeation (Myers, 1997).

\section{A.2.1 Standing Losses of Floating Roof Storage Tanks}

The standing losses from floating roof tanks are further sub-divided into standing losses associated with the floating roof's perimeter seal (known as deck rim seal loses) and the standing losses associated with all the combined losses from operational fittings that penetrate the floating roof (known as deck fitting losses).

\section{Deck rim seal losses}

Deck rim seal losses occur due to many complex mechanisms that vary with the different deck rim seal designs. However, it has been documented for EFRTs that the bulk of the deck rim seal losses are wind-induced (Myers, 1997). While the fixed outer roof of the IFRT minimizes the effect of cross-wind flow, crosswinds can still promote evacuation of the emissions from the head space of the IFRT (between the fixed roof and internal floating roof). Other non-dominant mechanisms that have been noted to promote losses through the deck rim seals are variations in the operating temperature and pressure. Various designs of the EFRT deck rim seals can be seen in Figure A.1(Myers, 1997; EPA, 1995) 

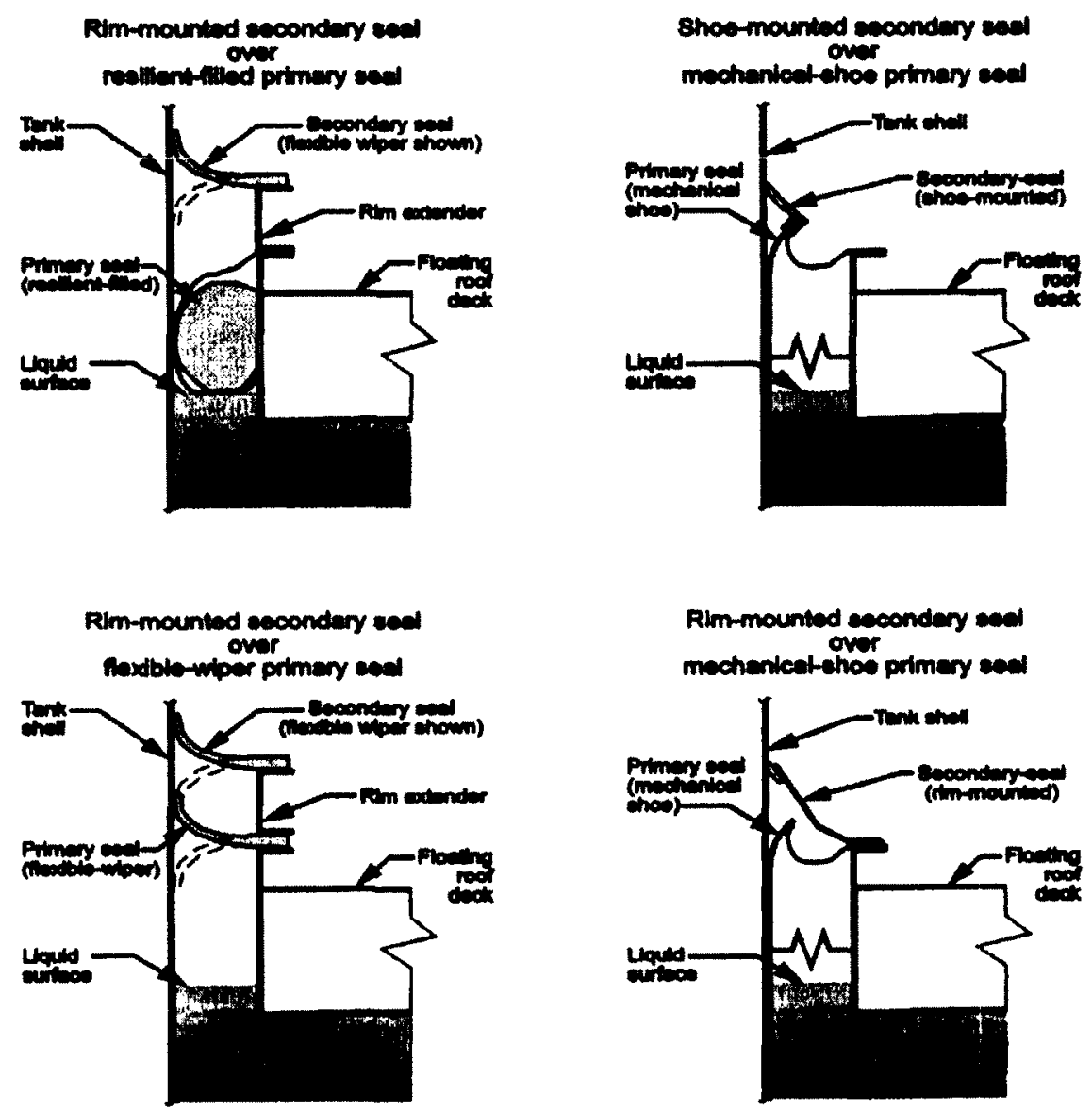

Figure A.1: Deck rim seals (EPA, 1995)

The deck rim seals, seen in Figure A.1, are broken down into two main sections; the primary and secondary seals. The primary seal encloses the exposed organic liquid in the annular space between the tank shell and the floating roof, therefore inhibiting the emissions to the atmosphere (EPA, 1995). The three main types of primary seals are: resilient core seal, mechanical shoe, and wiper seal (only IFRT). Resilient core seals are constructed of an annular bladder filled with either liquid (petroleum distillate) or foam that is held above organic liquid by an elastomeric coated fabric envelope (EPA, 1995). The resilient core's ability to deform allows the seal to adapt itself to minor imperfections in the tank wall, providing a better overall seal. The resilient core can be mounted in two ways: liquid mounted (seen in Figure A.1 - top left) and vapour mounted. The liquid mounted resilient core seals are mounted directly on the organic liquid preventing the 
formation of a rim vapour space (volume of air exposed to the organic liquid). The vapour mounted resilient seal which does allow a rim vapour space is documented by EPA (1995) to allow the highest rim losses (for EFRT), roughly 9-40 times that of the liquid mounted rim for a wind speed of $5-15 \mathrm{mph}$ respectively. The mechanical shoe seal is comprised of a metal plate held in compression against the tank wall with a flexible fabric cover to enclose the rim vapour space. EPA (1995) reports that this seal allows roughly double the fugitive emissions when compared to the liquid mounted resilient seal for ERFTs. Primary wiper seals (only IFRT) allow emission loses on par with vapourmounted resilient seals (EPA, 1995).

The secondary seals are a continuous seal mounted on the upper-most part of the rim and act as a barrier against emissions that would come from the small openings or tears in primary seal. The secondary seals are generally manufactured from flexible polymeric materials. While this allows the seal to be held in compression against the tank wall providing a robust barrier, they deteriorate due to debris and exposure to the elements (Myers, 1997). To prolong the life of the secondary seals, weather shields can be incorporated to provide added protection. The weather shields are constructed of thin overlapping steel sheets that are pivoted to ride the tank wall at an inclined angle (EPA 1995).

Myers (1997) states that the deck rim seals used for IFRT generally incorporate either a resilient foam-filled seal or wiper seal, similar to those of EFRT, as the primary seal. Since IFRTs are not significantly affected by the surrounding conditions (more specifically crosswind flow), the deck rim seal is mainly designed to compensate for small irregularities in the tank wall (EPA, 1995). The secondary seal is achieved through the implementation of a foam or wiper seal above the primary seal. While secondary seals for IFRTs may be used to provide added protection against emission losses, they are not commonly used unless specifically stated by the EPA's pollution control standards determined by the organic liquid being stored (EPA, 1995). 


\section{Deck fitting losses}

Other documented sources of evaporative losses are the various deck fittings that penetrate the floating roof allowing for vapour exchange between the organic liquid and the surroundings. The most common fittings that contribute to evaporative losses are:

- Access hatches

- Gauge float wells

- Gauge hatch/Sample ports

- Rim vents
- Deck drains

- Slotted unslotted guide poles

- Ladder Wells

- Vacuum Breaker

The design of each type of deck fitting can be seen in Figure A.2, Figure A.3 and Figure A.4. The access hatch is an opening in the floating roof deck with a peripheral well that penetrates the floating roof. They are typical sized to allow access to workers or required materials during construction and maintenance of the floating roof tank. These openings are closed using a removable cover that can either be left resting on the opening or bolted and/or gasketed to reduce evaporative losses from the vapour head space, located in the well (Myers, 1997; EPA, 1995)

A gauge float is used to determine the liquid level in the storage tank. It is a buoyant float that lies on the liquid surface and is connected to an indicator on the exterior of the tank via a cable and guide system. The gauge float well (where the gauge is housed) employs a sliding cover that moves with the floating roof and employs a bushing to allow dynamic penetration of the cable through the sliding cover. Emissions from the gauge float well can be reduced by bolting and/or gasketing the cover to the well wall (Myers, 1997; EPA, 1995).

Gauge hatch/sample ports consist of a pipe sleeve that penetrates the floating roof allowing access to the organic liquid being stored within the tank. The top of the pipe sleeve exposed to the surrounding is enclosed using a self-closing gasketed cover. The gauge sample ports are sized to allow hand sampling or sampling from the gauger's platform (Myers, 1997; EPA, 1995).

The evaporative losses that occur from these fittings are determined by the ability (or more specifically the inability) of the sealing technology implemented to prevent 
interaction between the atmosphere and the vapour space created by the deck fitting (located within the well). This is also true for fixed-roof support column wells used for IFRTs; however the bulk of the losses from support columns come for clingage losses described in Section A.1(EPA, 1995).

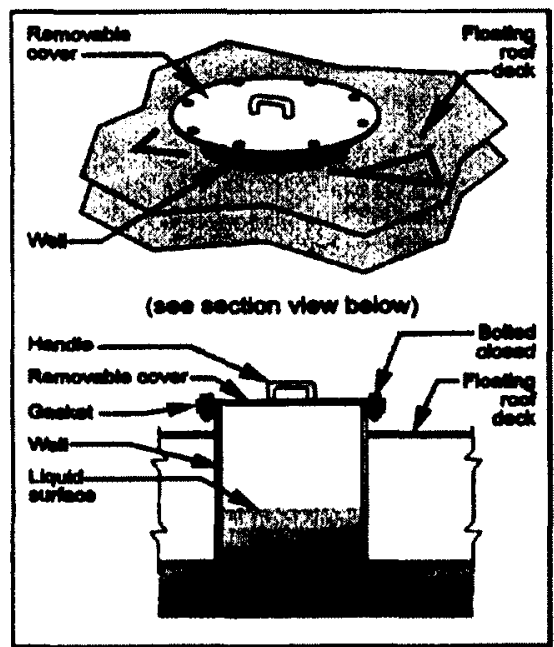

Acose Heloh

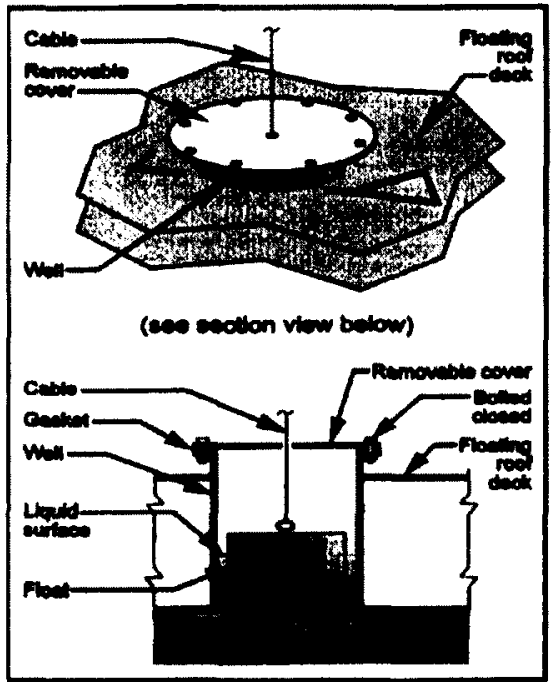

Couge fion

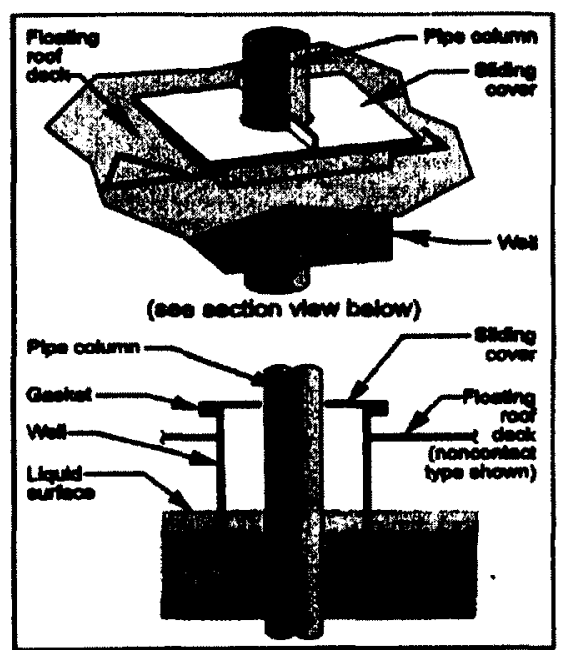

Fbad-Root Eupport Column

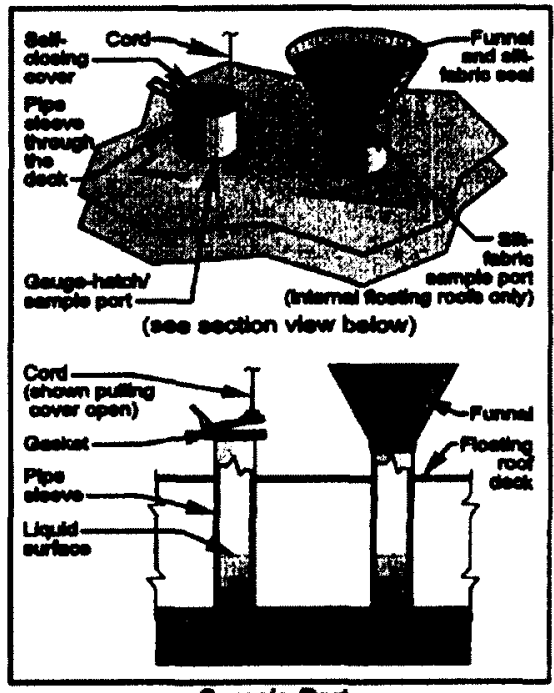

Qenple Port

Figure A.2: Typical Floating Roof Deck Fittings (EPA, 1995)

Vacuum breakers, in Figure A.3, are employed to equalize the pressure of the vapour space by allowing the exchange of vapour and air during the filling and emptying of the storage tank. A typical vacuum break consists of a well that penetrates the deck to the liquid level with a cover. The cover is attached to an adjustable leg of sufficient 
length, which comes into contact with the tank floor as the floating roof approaches the minimum set height, opening the cover of the vacuum breaker creating a opening for free exchange between the newly created vapour space (under the roof) and the surroundings. This configuration is known as a "landed roof". While this prevents drastic pressure variations within the tank, it contributes to the secondary form of standing storage evaporative loss called landing losses (Myers, 1997; EPA, 1995).

Deck drains, seen in Figure A.3, are essentially used to remove precipitation (rainwater, snow runoff etc.) from the surface of the floating roof. There are two main types of drainage systems, open and closed systems. The closed system carries the precipitation from the surface of the tank, via a deck drain fitting, to the outside of a tank via a flexible piping system that extends through the storage liquid space. Due to the separated nature of the closed drainage system, these deck fittings are documented to have almost no interaction with the stored product and therefore are not a source of evaporative losses (EPA, 1995). The open drainage system consisting of an open pipe that extends from the top of the floating roof into the organic liquid product space. The two most common types of open drainage systems are the flush drain and the overflow drain, seen in Figure A.3. Overflow drains are sometimes used as an emergency drainage system to prevent the accumulated precipitation from reaching unsafe levels. The open drainage systems can modified by placing an insert to mitigate evaporative losses from the organic liquid being stored (Myers, 1997; EPA, 1995).

Deck legs, as seen in Figure A.3, are used to prevent damage to the fittings present beneath the deck. They are comprised of steel poles that are held perpendicular to the deck via a deck sleeve. This also allows the roof to be suspended over tank floor, allowing for maintenance underneath the floating roof. The deck legs are shorter than the vacuum breaker legs so that the latter can trigger the vacuum breakers to open mechanically when the roof is "landed" (EPA, 1995). 


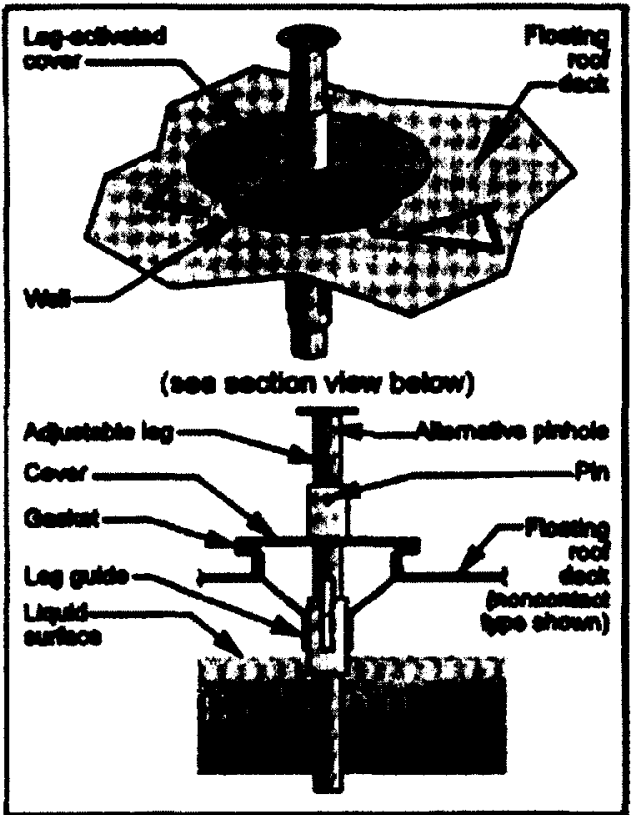

Vouum Breaker

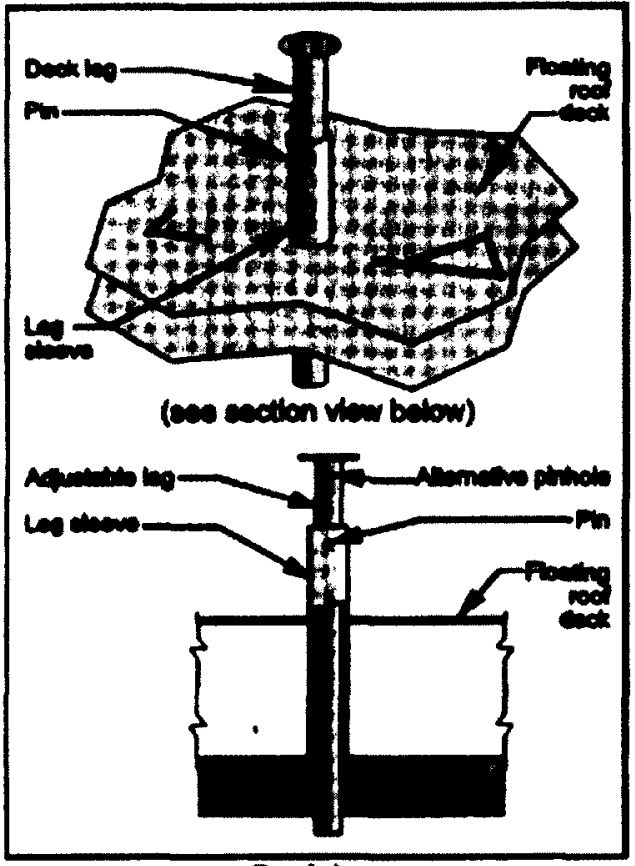

Deck LeO

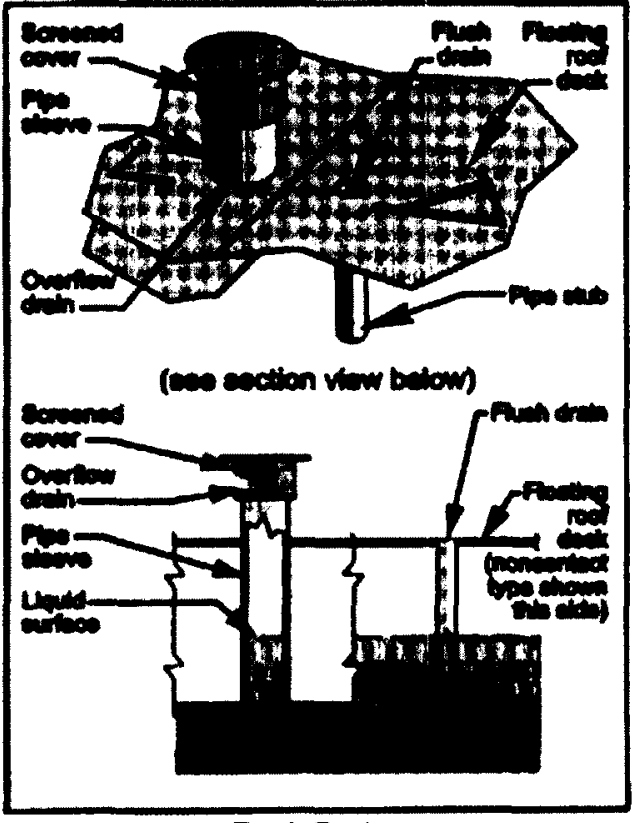

Deck Drine

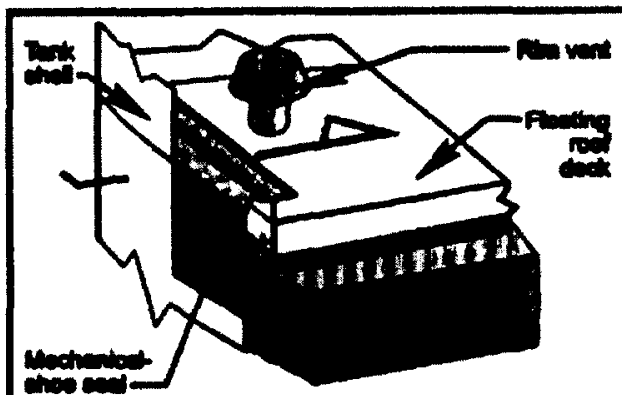

(cese cection viow below)

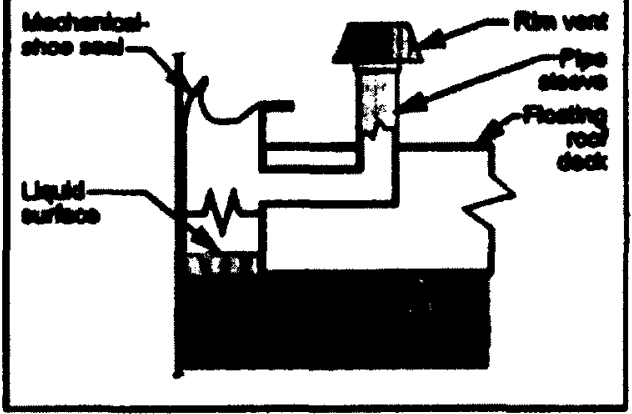

Rin Vent

Figure A.3: Typical Floating Roof Deck Fittings (EPA, 1995)

Anti-rotational deck fittings, known as guide poles are comprised of a vertical column that are held fixed at the base of the tank. These guide poles apply anti-rotational torques to the floating roof via the guide pole well assembly. The two main types are 
unslotted and slotted guide poles. The makeup of the unslotted guide pole can be seen in Figure A.4. The guide pole well assembly is comprised of a well cover and a roller assembly that ride the outside surface of the guide pole. In addition to the well cover, a sliding cover is used to allow very limited radial travel (perpendicular to rotation direction of the roof). To reduce evaporative losses from the guide pole assemble, the guide pole cover and the float within the guide pole may be equipped with gaskets, ensuring a better seal. To mitigate evaporative losses from the guide pole assembly, a pole sleeve that extends from the cover to below the liquid level can be inserted. This prevents any vapour exchange between the well's vapour space and the internal space of the slotted guide pole (Myers, 1997; EPA, 1995)

The evaporative losses from these fittings, which are further amplified by operational wear and meteorological conditions, make fugitive emissions from floating roof tanks a significant pollutant contributor in the oil and gas industry (Chambers et al., 2006).

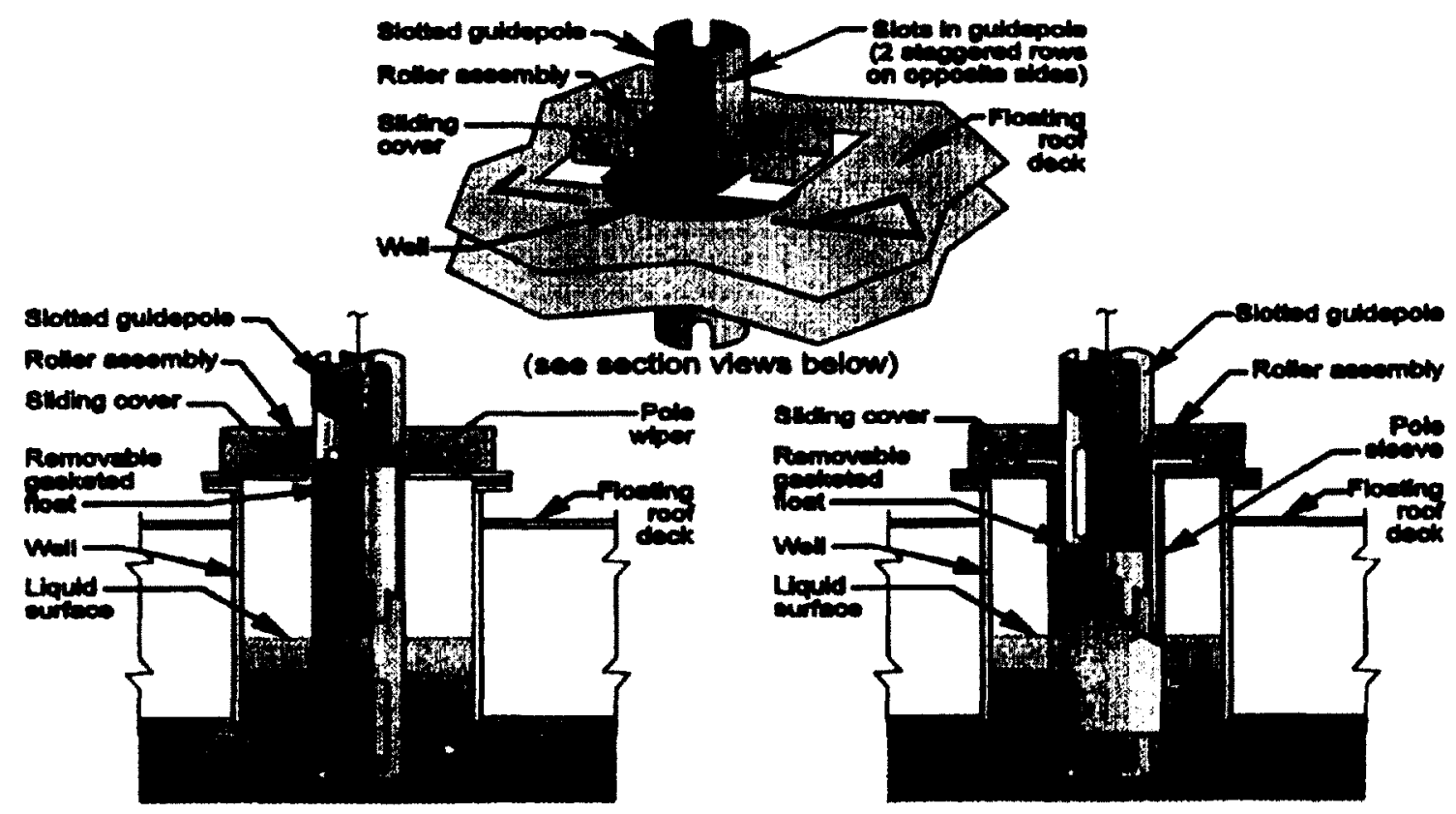

Figure A.4: Slotted guide pole deck fitting (EPA, 1995) 


\section{Standing Losses for Landed Floating Roofs}

As stated earlier landing losses occur after the floating roof reaches its minimum height and the vacuum breakers are mechanically engaged to equalize the pressure under the floating roof as the liquid continues to drop, leading to landing losses. These losses are amplified in EFRTs by crosswind flow which continually drives evaporative losses (EPA, 1995). The three main categories of a landed roof can be seen in Figure A.5. The breathing losses from a landed roof will remain dominant so long as there exists, wetted areas across the floor of the tank. Furthermore, during the initial filling of the tank (when the roof is landed), the addition of more liquid stock will displace the "heavier" (nonbuoyant) hydrocarbon vapours present under the deck further promoting evaporative losses (Myers, 1997; EPA, 1995).

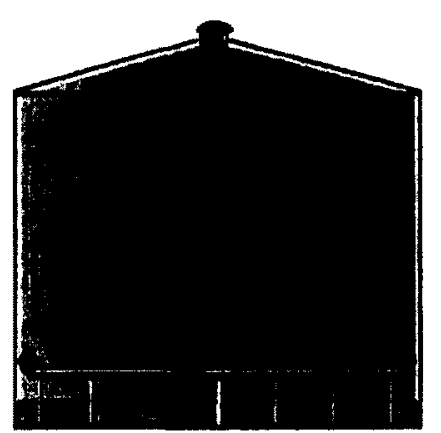

Full Liquid Heel

(standing liquid across the entire bottom)

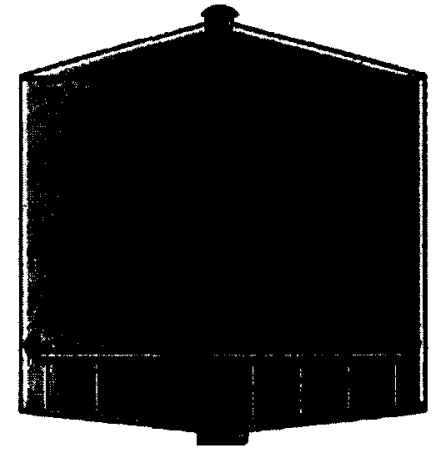

Partial Liquid Heel (standing liquid only in or near a sump; clingage elscwhere)

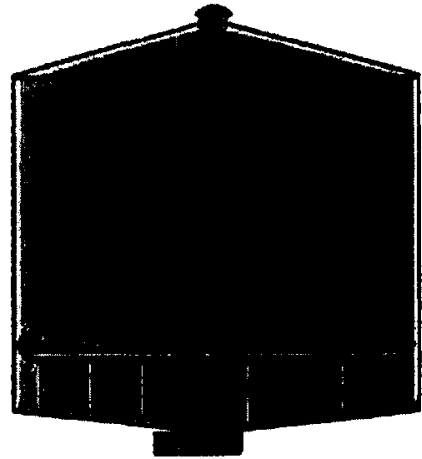

Drain Dry (no standing liquid, only liquid is clingage)

Figure A.5: Different configurations of a "landed" internal roof (EPA, 1995)

\section{A.2.2 Standing Losses of Fixed Roof Tanks and Horizontal Tanks}

The standing losses for fixed roof and horizontal tanks are limited to sample ports, roof access holes, and breather vents. Myers (1997) indicates that generally most breather vents are/or have been modified to include pressure/vacuum valves, but if the breather vent is simply left open to atmosphere, all other standing losses are negligible in comparison. The sample port and access hole fittings for fixed roof tanks and 
aboveground tanks are similar to the EFRT counterparts and are sealed in similar fashions (bolted/gasket) and are thereby susceptible to the same atmospheric losses caused by wind flow and operational wear. Standing losses for underground horizontal tank are solely comprised of venting losses if the vent is not fitted with a pressure/vacuum fitting. Standing losses for underground tank fittings are nearly nonexistent due to the waterproof seals in place on the opening of passageways to the access holes/ fuel line/ fill pile, seen in Figure A.6. Additionally, EPA (1995) suggests that the diurnal temperature variations that cause pressure changes (leading to pressure/vacuum venting losses), are almost non-existent due to the isothermal behaviour of the surrounding earth. However, it is worth noting that recent field measurements led by Clearstone Engineering Ltd. in collaboration with Carleton University, suggest that this may not actually be true if entrained air is significantly cooler than the ground temperature (Clearstone Engineering Ltd., 2011).

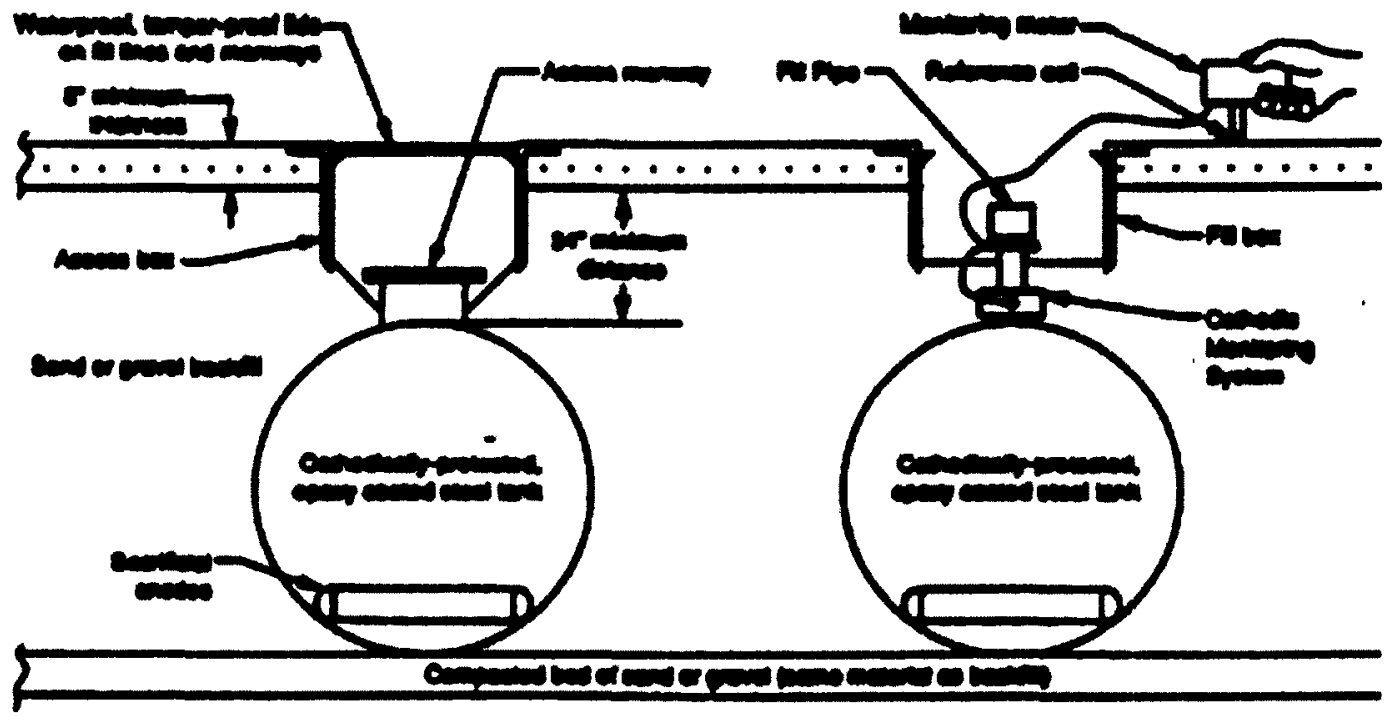

Figure A.6: Section view of a typical underground horizontal storage tank (EPA, 1995) 


\section{Appendix B}

\section{Current Models for Estimating Emissions from Liquid Storage Tanks}

Current models for estimating evaporative losses from liquid storage tanks are based on a series of semi-empirical algorithms developed through work cooperatively sponsored by the American Petroleum Institute (API) and the U.S. Environmental Protection Agency (EPA), and are detailed in Chapter 7.1 of AP-42 (EPA, 1995). Through the effort of EPA's office of air quality and standards in technology transfer networking, the algorithms have been published as a freeware emission-calculating program known as TANKS (current version is 4.09) (EPA, 1995).

EPA TANKS is a Windows based computer program that allows for the user to estimate the annual average emissions when the tank characteristics, turnover per year, average vapour pressure, and average ambient temperature are specified. The flashing losses described in Chapter 1 are not taken into account in AP-42 emission estimates. This is because the estimates were designed to represent the storage of stable liquids (i.e. all liquid) and therefore does not account for trapped hydrocarbon gasses within the organic liquid that "flash" out during the filling cycle (EPA, 1995).

\section{B.1 Emission Factors of Floating Roof Tanks}

The current emission estimating algorithms for floating roof tanks are presented here in three parts: standing losses, working losses and landed roof losses. For floating roof tanks, the working losses are solely comprised of the clingage losses (for EFRT) or filling losses (for IFRT) due to change of the liquid level of the tank. For an EFRT, as the liquid level is reduced, the floating roof descends and exposes the tank's inner "wetted" surface to the atmosphere, allowing for ("clingage") evaporative losses. For an IFRT, a change in 
the level of the internal floating roof induces an exchange of gases between the vapour space above the floating roof (within the tank) and the surroundings, since the fixed external roof does not sustain a pressure difference and must exchange gas with the ambient environment to regulate the internal pressure. During the filling cycle of an IFRT, any hydrocarbon vapours above the internal floating roof are displaced through vents in the fixed outer roof into the atmosphere.

The standing losses (also known as breathing losses) are broken down into two main groups, the losses from the deck rim seal and those of the multiple deck fittings that penetrate the deck. The fugitive emission estimates from standing losses do not take into to account long term transients such as wear and destruction of gasket seals or fittings, which would contribute to higher wind induced emissions (EPA, 1995).

The total annual evaporative losses are estimated by the relation presented in Equation B.1. The evaporative losses that are generated when the floating roof is landed are separately considered, since those losses are estimated on a per episode basis and differ depending on the landed roof scenarios (EPA, 1995).

$$
L_{T}=L_{R}+L_{W D}+L_{F}+L_{D}
$$

where:

$L_{T}$ is the total evaporative losses $[\mathrm{lb} / \mathrm{yr}]$,

$L_{R}$ is the rim seal evaporative losses [ $[\mathrm{b} / \mathrm{yr}]$,

$L_{F}$ is the deck fitting evaporative losses [lb/yr],

$L_{W D}$ is the evaporative loss due to withdrawal [lb/yr], and

$L_{D}$ is the deck seam losses (IFRT's only) [lb/yr]

\section{B.1.1 Standing loses of Floating Roof Tanks}

Rim seal losses can be estimated via Equation B.2 (EPA, 1995):

$$
L_{R}=\left(K_{R a}+K_{R b} \cdot v^{n}\right) D \cdot P^{*} \cdot M_{V} \cdot K_{C}
$$

where:

$K_{R a}$ is the zero wind speed rim seal loss factor [lb-mole/ft-yr], 
$K_{R b}$ is the wind dependent rim seal loss factor [lb-mole/(mph $\left.)^{\mathrm{n}}-\mathrm{ft}-\mathrm{yr}\right]$, $v$ is the average ambient wind speed at tank site (is zero for IFRT) [mph],

$n$ is the seal related wind speed exponent fitting evaporative loss [-],

$D$ is the storage tank diameter [ $\mathrm{ft}$ ],

$M_{V}$ is the average molecular weight of the liquid stock [lb/lb-mole],

$K_{C}$ is the product factor [-] ( 0.4 for crude oils; 1 for all other organic liquids), and

$P^{*}$ is the vapour pressure function [-] (see Equation B.3 for further details).

The wind dependent behaviour of Equation B.2 was determined through experimental testing at equilibrium conditions and at fixed wind speeds (from 2.2 to $13.1 \mathrm{mph}$ ) (EPA, 1995). In these tests, hydrocarbon measurements were conducted downstream at steady state conditions to determine the release rates for each test condition and based on these data, the annual emission rate was determined assuming an annual average wind speed. However, since the wind speed is exponentially related to the standing losses, the error in the estimate also grows in a similar fashion, as determined by a sensitivity analysis conducted by EPA (1995).

From the same sensitivity analysis it was determined that the error introduced by using an average wind speed rather than averaging the standing loses over the annual period that uses the annual wind speed distribution, is dependent on the dimensionless wind speed exponent (as seen in Equation B.2) and the coefficient of variation (i.e. the ratio between standard deviation to the mean) of the wind speed (EPA, 1995). It was found that for a coefficient of variation of 0.45 to 0.6 , the error induced for wind speed exponents of 0.4 and 1.5 were between 2 to $5 \%$ and 7 to $11 \%$ respectively. However for a wind speed exponent of 2.6, the error induced for the same coefficient of variation range, was found to be 30 to $45 \%$ (EPA, 1995). For wind speed exponents greater than the 1.5, EPA (1995) recommended that a detailed wind speed distribution be taken into account. See Table B.1, for additional details on $K_{R a}$ and $K_{R b}$ factors (EPA, 1995). 
Table B.1: Rim seal loss factors (EPA, 1995)

\begin{tabular}{|c|c|c|c|}
\hline \multirow{2}{*}{$\begin{array}{l}\text { Tank Construction And } \\
\text { Rim-Scal System }\end{array}$} & \multicolumn{3}{|c|}{ Average-Fitting Seals } \\
\hline & $\begin{array}{c}K_{R A} \\
\text { (lb-mole/ft-yr) }\end{array}$ & $\underset{\left[l b-m o l e /(m p h)^{n}-n t-y r\right]}{K_{R b}}$ & $\stackrel{n}{n}$ \\
\hline \multicolumn{4}{|l|}{ Welded Tanks } \\
\hline \multicolumn{4}{|l|}{ Mechanical-shoe seal } \\
\hline Shoc-mounted secondary & 1.6 & 0.3 & 1.6 \\
\hline Rim-mounted secondary & 0.6 & 0.4 & 1.0 \\
\hline \multicolumn{4}{|l|}{ Liquid-mounted seal } \\
\hline Primary only & 1.6 & 0.3 & 1.5 \\
\hline Weather shield & 0.7 & 0.3 & 1.2 \\
\hline Rim-mounted secondary & 0.3 & 0.6 & 0.3 \\
\hline \multicolumn{4}{|l|}{ Vapor-mounted seal } \\
\hline Primary only & 6.7 & 0.2 & 3.0 \\
\hline Weather shield & 3.3 & 0.1 & 3.0 \\
\hline \multirow[t]{2}{*}{ Rim-mounted secondary } & 2.2 & 0.003 & 4.3 \\
\hline & Riveled Ta & nks & \\
\hline \multicolumn{4}{|l|}{ Mechanical-shoe seal } \\
\hline Primary only & 10.8 & 0.4 & 2.0 \\
\hline Shoe-mounted secondary & 9.2 & 0.2 & 1.9 \\
\hline Rim-mounted secondary & 1.1 & 0.3 & 1.5 \\
\hline
\end{tabular}

Empirical data showed that standing losses have a reasonably linear relationship to the tank diameter, i.e. the diameter exponent parameter varied between 0.9 and 1.2 (EPA, 1995). However, EPA (1995) utilizes an exponent of one, which introduces additional uncertainty of the tank diameter exponent. The uncertainty of the calculated evaporative losses grows substantially as the tank diameter increases which in turn results in sizable variation in the calculated emissions for large sized tanks (EPA, 1995). For example, a $100 \mathrm{ft}$ tank yields an uncertainty band of around 25 to 250 percent of the estimated standing loss while the uncertainty error of the tank diameter exponent is minimal $(\sim 1-5 \%)$. On the other hand, the uncertainty of the tank diameter exponent is substantially large for the smaller storage tanks (up to $20 \%$ percent deviation in a particular some cases), but leading to small uncertainties in the calculated losses (up to 10 percent in a particular case) (EPA, 1995).

The vapour pressure dimensionless parameter can be calculated via Equation B.3.

$$
P^{*}=\frac{P_{V A} / P_{A}}{\left[1+\left(1-\frac{P_{V A}}{P_{A}}\right)^{.5}\right]^{2}}
$$


where:

$P_{V A}$ is the vapour pressure at daily average liquid surface temperature [psia], and $P_{A}$ is the atmospheric pressure [psia].

The vapour pressure is highly dependent on the operating temperature and molecular composition of the crude stock. The operating temperature is subject to continual changes through heat transfer from the surroundings. Likewise, the molecular composition of the (unrefined) crude stock is subject to change for every oil well tapped. Consequently, these variations in temperature and molecular composition cause the evaporative losses to vary in a similar fashion. For example, a $\pm 20^{\circ} \mathrm{F}$ deviation in temperature will result in a standing loss variation of 40 percent (for $-20^{\circ} \mathrm{F}$ deviation) or 65 percent (for $+20^{\circ} \mathrm{F}$ deviation) for some cases as documented by EPA (1995). Since the average annual operating temperature is used to estimate the evaporative losses, the deviations in the evaporative losses estimate are said to average out over time (without definite proof) (EPA 1995).

To calculate the evaporative losses, if the operating temperature of the crude oil is unknown (i.e. not measured), AP-42 suggests a bias temperature correction to the ambient temperature based on the estimated heat transfer to the tank from the surroundings. While this procedure allows the user to determine the stored liquid's temperature for the purposes of calculating the standing losses of the storage tank, care should be taken due to the increase uncertainty of the estimate (EPA, 1995).

The standing losses from all the deck fittings can be estimated by the following equation (EPA, 1995).

$$
L_{F}=F_{F} \cdot P^{*} \cdot M_{V} \cdot K_{C}
$$

where:

$F_{F}$ is the total deck fitting loss factor [lb/yr] (see Eq. B.5 for further detail),

$P^{*}$ is the vapour pressure function [-] (see Eq. B.3 for further detail),

$M_{V}$ is the average molecular weight of the liquid stock [lb/lb-mole], and

$K_{C}$ is the product factor [-] ( 0.4 for crude oils; 1 for all other organic liquids). 
The total deck fitting loss factor is essentially a summation of all the deck fittings individual contributions which can be seen in the equation below. For the compilation of all the different types of deck fittings and their corresponding loss factors, refer to Table 3.1-12 of chapter 7.1 of AP-42 (EPA, 1995).

$$
F_{F}=\sum_{i=0}^{n_{f}}\left(N_{F i} \cdot K_{F i}\right)
$$

where:

$i$ is the number of a particular type of deck fitting $\left(i=0,1,2, \ldots, n_{f}\right)$,

$n_{f}$ is total number of different types of fittings,

$N_{F i}$ is the number of deck fittings of type $i\left(i=0,1,2, \ldots, n_{f}\right)$, and

$K_{F i}$ is deck fitting loss factor for the deck fitting of type $i\left(i=0,1,2, \ldots, n_{f}\right)$ [lb-mole/yr].

The deck fitting loss factor can be estimated from the following equation (EPA, 1995).

$$
K_{F i}=K_{F a_{i}}+K_{F b_{i}} \cdot\left(K_{V} \cdot v\right)^{m_{i}}
$$

where:

$K_{F a_{i}}$ is the zero wind loss factor for a particular type of fitting $i$ [1b-mole/yr],

$K_{F b_{i}}$ is the wind dependent loss factor for a particular type of fitting $i$

$$
\text { [lb-mole/(mph })^{\mathrm{m}} \text {-ft-yr], }
$$

$K_{V}$ is the fitting wind speed correction factor [-],

$v$ is the average ambient wind speed (zero for IFRT)[mph], and

$m_{i}$ is the loss factor for a particular type of fitting $i[-]$.

The determination of the fitting loss factors were developed by EPA (1995) through control wind tunnel testing of each type of deck fitting mounted on a product reservoir, at constant wind speeds of $0,4.3,8.5$ and $11.9 \mathrm{mph}$. Except for the deck leg and deck drain fittings, all of the deck fittings were tested with and without an additional gasket seals. The wind speed emission coefficients $K_{F a_{i}}, K_{F b_{l}}$ were determined by two main techniques. The first utilized a least squares iterative algorithm to force fit the 
nonlinear relation to the measured emissions and more importantly, to force fit the relation to the zero wind speed measurement $\left(K_{F a_{i}}\right)$ by assigning it a significantly larger weight (EPA, 1995). The second method, which provided similar estimates but is noted by EPA (1995) to have better precision, subtracts the zero wind speed reading from all other data points, thereby removing the bias (non-wind dependent) emissions from the wind dependent losses. The uncertainty of the losses calculated utilizing the deck fitting factors were determined to be on the same order of magnitude as the losses themselves (EPA, 1995). The high uncertainty is documented to be a possible effect of the small sample size or the inherent variability in the emissions generated by deck fittings. Factors such as wind direction and non-symmetric fitting geometry are noted to lead to sizable variability of the calculated loses (EPA, 1995).

When comparing the ambient wind speeds to the local wind speeds around the deck fitting, the fitting wind speed correction factor (of 0.7 ) for an external floating roof tanks is used to represent the shielding effect caused by the tank walls. The speed correction factor $K_{V}$, was developed by EPA (1995) through extensive wind tunnel testing, where the horizontal wind speed component across the tank was measured for the different roof height to tank height ratios $(R / H)$. By conducting studies on both the nominal operating time spent in each $\mathrm{R} / \mathrm{H}$ ratio condition and the associated speed zones across the deck, the weighted average speed correction factor was determined to be 0.45 for the outer annular area and 0.53 for the inner area of the deck. However, these factors were developed without accounting for the vertical component of the local wind (EPA, 1995). This was made apparent when the effect of vertical wind speeds on the deck fitting's evaporative loses was brought into question by a wind speed sensitivity analysis conducted by EPA (1995). To compensate for this oversight, EPA (1995) used field wind data, direct vector addition of the Cartesian components ( $x$ and $y$ ) and the weighted averages of the deck wind zones to develop a new wind speed correction factor of 0.7 , which is the current wind speed correction factor recommended by API. A key point of interest is that the accuracy of the field data has been mentioned by EPA (1995) as "questionable", due to low sample size and the variation of the deck wind speed 
measurements, some found to be 10 times greater that the ambient wind speed raising concerns of the noise/error of the measurement devices. The error statistics of these devices were not reported to EPA (1995).

The deck seam losses, only present for IFRTs with bolted decks are estimated by the following relation.

$$
L_{D}=K_{D} \cdot S_{D} \cdot D^{2} \cdot P^{*} \cdot M_{V} \cdot K_{C}
$$

where:

$K_{D}$ is the deck seam loss per unit seam length factor[lb-mole/ft-yr] ( 0 for welded decks; 0.14 for bolted decks), and

$S_{D}$ is the deck seam factor (total length of seams / area of the deck) $\left[\mathrm{ft} / \mathrm{ft}^{2}\right]$.

While the uncertainty of the evaporative losses in regards to the deck seam factors $\left(K_{D}, S_{D}\right)$ have not been documented by EPA (1995), the uncertainty caused by $P^{*}$, $M_{V}$ and $K_{C}$ behave in a similar fashion to those presented in Equation B.2 \& B.3, i.e. temperature and organic molecular variation (EPA,1995).

\section{B.1.2 Working losses of Floating Roof Tanks}

The working evaporative losses (more specifically the withdrawal losses) can be estimated via Equation B.8. For further details on clingage factors, see Table B.2, which summarizes the effect on withdrawal losses from the inner tank shell surface roughness and the organic composition of the stored liquid (EPA, 1995).

$$
L_{W D}=\left(\frac{0.943 \cdot Q \cdot C_{S} \cdot W_{L}}{D}\right)\left[1+\frac{N_{C} \cdot F_{C}}{D}\right]
$$

where:

$Q$ is the annual throughput [barrels/yr],

$C_{S}$ is the tank shell clingage factor [barrels $/ 1000 \mathrm{ft}^{2}$ ],

$W_{L}$ is the average organic liquid density [lb/gal],

0.943 is the empirical constant for withdrawal losses $\left[1000 \mathrm{ft}^{2}\right.$-gal/barrels $\left.{ }^{2}\right]$, $N_{C}$ is the number of fixed roof support columns (for IRFT, 0 for EFRT) [-], and 
$F_{C}$ is the effective column diameter [ft].

Table B.2: Clingage factors (EPA, 1995)

\begin{tabular}{|c|c|c|c|}
\hline \multirow{2}{*}{ Product Stored } & \multicolumn{3}{|c|}{ Shell Surface Condition } \\
\cline { 2 - 4 } & Light Rust & Dense Rust & Gunite Lining \\
\hline Gasoline & 0.0015 & 0.0075 & 0.15 \\
\hline $\begin{array}{c}\text { Single-Component } \\
\text { Stocks }\end{array}$ & 0.0015 & 0.0075 & 0.15 \\
\hline Crude oil & 0.0060 & 0.03 & 0.60 \\
\hline
\end{tabular}

From Table B.2, it can be seen that the surface roughness (i.e. shell condition) of the inner tank wall plays a sizable role in the amount of emissions released. The working losses are seen to vary over time due to weather induced degradation of the tank wall, continually increasing over the lifespan of the tank (shell). Additionally, the annual throughput $(\mathrm{Q})$ is not a full representation of the frequency at which the external floating roof moves vertically. More specifically, while some tanks a have high throughput, the roof height is maintained relatively constant, or vice versa. These cases should be dealt with accordingly, by calculating the emissions based on the average change in liquid height, or by using default turnover rates that are representative of the tank's behaviour (EPA, 1995)

\section{B.1.3 Fugitive Emission Factors of "Landed" Floating Roof Tanks}

The evaporative losses of a landed floating roof are determined by the liquid level of the floating roof (i.e. full liquid heel or drain dry) and are comprised of two main parts, standing landed losses and filling losses.

The standing losses are primarily estimated as wind induced breathing losses from the deck seals (rim and fitting) and the open pathways created by the vacuum breakers that allow for pressure equalization during the "landed" operating condition of the floating roof. The three driving mechanism are: breathing losses, wind losses and clingage losses.

The filling losses are essentially driven by the displacement of the vapour under the floating roof by incoming organic liquid during the filling process. Since the total 
time per annum the roof is said to be in the "landed" configuration is highly variable, the estimates can only be generated on a per episode basis. In addition, the liquid level per episode is subject to change and by that effect will change the standing loss relations that need to be utilized in order to properly represent the emissions release per landing episode (EPA, 1995).

The total evaporative loss from floating roof tanks during a landing episode is given by Eq. B.9, and is essentially a summation of the standing landed losses and the filling losses (EPA, 1995).

$$
L_{T L}=L_{S L}+L_{F L}
$$

where:

$L_{T L}$ is the total losses during roof landing, [lb per episode], $L_{S L}$ is the standing losses during roof landing, [lb per episode], and $L_{F L}$ is the filling losses during roof landing, [lb per episode].

As previously stated, standing losses during a roof landing are determined by the amount of organic liquid available (i.e. either liquid heel or drain-dry) for evaporation. For a liquid heel landed roof, the primary mechanisms are broken down into: breathing losses, wind losses and clingage losses. For landed IFRTs, the breathing losses are identical to those seen with fixed roof tanks. The stored organic liquid evaporates directly into the vapour headspace (between the internal floating roof and the fixed roof) which is contained by the breather vent. However, temperature and barometric variations cause pressure variations that exceed the breather vent's relief pressure (typically $0.3 \mathrm{psi}$ ), resulting in breathing losses to the surroundings (EPA, 1995). Alternatively for external floating roof tanks, temperature and pressure changes have little contribution to the total evaporative losses when compared to the wind driven evaporative losses. The open configuration caused by the vacuum breakers allows the wind to evacuate vapour directly above the liquid surface (below the floating roof). The evaporative losses of landed IFRTs and ERFTs with the liquid heel configuration can be seen in Equation B.10 and B.11 respectively (EPA, 1995). 


$$
\begin{gathered}
L_{S L-L H-I F R T}=n_{d} \cdot W_{V} \cdot V_{V} \cdot K_{E} \cdot K_{S} \\
L_{S L-L H-E F R T}=0.57 \cdot n_{d} \cdot D \cdot P^{*} * M_{V}
\end{gathered}
$$

where:

$L_{S L-L H}$ is the daily standing evaporative losses due to wind for a landed roof with a liquid heel [lb],

$n_{d}$ is the number of days that floating roof remains landed with a liquid heel [days],

$W_{V}$ is the stock vapour density $\left[\mathrm{lb} / \mathrm{ft}^{3}\right]$,

$V_{V}$ is the volume of the vapour headspace $\left[\mathrm{ft}^{3}\right]$,

$K_{E}$ is the stock vapour expansion factor (see Equation B.12) [-],

$K_{S}$ is the saturation factor [-],

$D$ is the tank diameter [ $\mathrm{ft}$ ],

$P^{*}$ is the vapour pressure function [-] (see Eq. B.3 for further detail), and

$M_{V}$ is the average molecular weight of the liquid stock [lb/lb-mole].

$$
K_{E}=\left(\frac{\Delta T}{T}\right) \cdot\left(1+\frac{0.5 \cdot B \cdot P}{T \cdot\left(P_{A}-P\right)}\right)
$$

where:

$\Delta T$ is the daily temperature operating range $\left[{ }^{\circ} \mathrm{R}\right]$,

$T$ is the average temperature of the vapour and the organic liquid $\left[{ }^{\circ} \mathrm{R}\right]$,

$B$ is the empirical constant of the vapour pressure equation $\left[{ }^{\circ} \mathrm{R}\right]$,

$P$ is the true vapour pressure of the organic liquid [psia], and

$P_{A}$ is the average atmospheric pressure at the storage tank's location [psia].

The evaporative losses experienced by drain-dry landed storage tanks is said to behave in a similar fashion to clingage losses (EPA, 1995). However, the losses are estimated as a one-time event rather than a daily event because once the storage tank is emptied, evaporation is limited to the amount of liquid still clinging to the tank floor. Therefore, the losses are solely estimated by the evaporation of the "wetted" areas of the 
tank floor and thereby are proportional to the clingage factor as seen in B.8. The equation below is used to estimate the standing losses for a drain-dry EFRT (EPA, 1995).

$$
L_{S L-D D-E R F T}=42 \cdot C_{S} \cdot W_{l} \cdot \text { Area }
$$

where:

$L_{S L-D D}$ is the evaporative losses due to wind for a drain-dry landed roof [lb],

42 is the conversion factor [gal/bbl],

$C_{s}$ is the clingage factor $\left[\mathrm{bbl} / 1000 \mathrm{ft}^{2}\right]$,

$W_{l}$ is the density of liquid [lb/gal], and

Area is the surface area of the tank floor $\left[\mathrm{ft}^{2}\right]$.

The filling loses of FRTs are determined by the amount of "heavy" vapour still present within the vapour space underneath the floating roof. The filling losses are determined by two main things, the volume of vapour that is be displaced by the incoming liquid to the point the roof is no longer considered "landed" and the percent of the vapour that is saturated with vaporized hydrocarbons (EPA, 1995). The filling losses that are associated with volume displacement are represented by $C_{s f}$ correction factor presented in Equation B.15. The percent of air that is saturated with hydrocarbons is determined by the liquid stock available for evaporation i.e. a full liquid heel has the highest potential for evaporation and a drain-dry tank has the lowest. To account for this EPA (1995) utilizes the saturation factor $(S)$. Since IFRT breather vents limit the evaporative losses to the atmosphere, the filling losses incurred by a drain-dry tank are said to be limited by maximum saturation possible, that is seen for filling losses with a full liquid heel, i.e. S equals 0.6 (see Equation B.14)(EPA, 1995). While this provides an upper limit, the uncertainties for this estimation have not been presented to prove the applicability of this relation.

The equation below is used to estimate the filling losses for both EFRT and IFRT (EPA, 1995).

$$
L_{F L}=\left(\frac{P \cdot V_{V}}{R \cdot T}\right) \cdot M_{V} \cdot C_{s f} \cdot S
$$


where:

$L_{F L}$ is the daily standing filling losses for a landed roof with a liquid heel [lb],

$P$ is the true vapour pressure of the organic liquid [psia],

$V_{V}$ is the volume occupied by the vapour space above the liquid heel $\left[\mathrm{ft}^{3}\right]$,

$R$ is the ideal gas constant $\left[10.731 \mathrm{psia}^{\mathrm{f}} \mathrm{ft}^{3} / \mathrm{lb}-\right.$ mole- $\left.{ }^{\circ} \mathrm{R}\right]$,

$T$ is the average temperature of the vapour and liquid below the floating roof $\left[{ }^{\circ} \mathrm{R}\right]$,

$M_{V}$ is the average molecular weight of the liquid stock [lb/lb-mole],

$S$ is the filling saturation factor [-];

0.6 for full liquid heel,

0.5 for partial liquid heel,

0.15 for drain-dry storage tank, and

$C_{s f}$ is the correction factor [-]; $C_{s f}=1$ for IFRT and drain dry EFRT.

The $C_{s f}$ factor is essentially a combination of standing loss relations presented earlier, used to quantify the decrease in hydrocarbon saturation of the displaced vapour caused by previous wind induced standing losses (EPA, 1995).

$$
C_{s f}=\left(\frac{\left(0.57 \cdot n_{d} \cdot D \cdot P^{*} * M_{V}\right)-\left(n_{d} \cdot K_{E} \cdot\left(\frac{P \cdot V_{V}}{R \cdot T}\right) \cdot M_{V} \cdot K_{S}\right)}{\left(n_{d} \cdot K_{E} \cdot\left(\frac{P \cdot V_{V}}{R \cdot T}\right) \cdot M_{V} \cdot K_{S}\right)+\left(s \cdot M_{V} \cdot\left(\frac{P \cdot V_{V}}{R \cdot T}\right)\right)}\right)
$$

While EPA (1995) outlines a general methodology of calculating evaporative losses during the landed roof operating condition, detailed sensitivity analysis or comparisons to experimentally measured losses, to the author's knowledge have not been presented. This is in part due to the lack of documentation on the effects of critical parameters on landed loses, more specifically the effects of wind accelerated evaporative losses during a landed floating roof with a liquid heel configuration. Ferry (2002) indicated that the data from wind studies on EFRTs and the "open circuit" interaction of crude stock with the environment indicates that the sizable emission release during a roof landing episode have yet to be fully quantified. 


\section{B.2 Emission Factors of Fixed Roof Tanks and Horizontal Tanks}

The types of evaporative losses from fixed roof tanks and horizontal tanks are also categorized under either standing losses or working losses. Standing losses for fixed roof tanks (with pressure-breather vents) are determined by diurnal temperature and barometric variations (EPA, 1995). These changes cause vapour expansion or contraction which proportionally elevates or lowers the internal pressure (with no change in liquid level) of the tank causing differential pressure across the (pressure) breather vent. Once the relief set point is reached (typically $\pm 0.03 \mathrm{psig}$ as indicated by EPA (1995)), the breather vent allows vapour release to the surroundings to alleviate the pressure build up. Fixed roof tanks that utilize open-breather vents are also affected by crosswind flows that promote convective evaporative losses. Working losses as mentioned previously, account for the losses induced by change in the level of the stored organic liquid i.e. during the filling and emptying cycle. The total losses are the sum of the aforementioned components.

$$
L_{T}=L_{S}+L_{W}
$$

where:

$L_{T}$ is the total evaporative losses [lb/yr],

$L_{S}$ is the standing evaporative losses [lb/yr], and

$L_{W}$ is the working evaporative losses[lb/yr].

\section{B.2.1 Standing losses of Fixed Roof and Horizontal Tanks}

The fixed roof tank standing losses can be estimated from the following equation.

$$
L_{S}=365 \cdot\left(\frac{\pi}{4} \cdot D^{2}\right) \cdot H_{V O} \cdot W_{V} \cdot K_{E} \cdot K_{S}
$$

where:

$L_{S}$ is the standing evaporative loss [lb/yr]; 0 for underground horizontal tanks,

$D$ is the tank diameter [ $\mathrm{ft}$; for horizontal tanks the effective diameter is given by

$$
D=\sqrt{\left(\frac{4}{\pi} \cdot L \cdot D_{H}\right)}
$$

$L$ is the length of the horizontal tank [ft],

$D_{H}$ is the diameter of the horizontal tank[ft], 
$H_{V O}$ is the vapour space outage $[\mathrm{ft}] ;\left[\frac{\pi D_{H}}{8}\right]$ for horizontal tanks,

$W_{V}$ is the vapour space density $\left[\mathrm{lb} / \mathrm{ft}^{3}\right]$,

$K_{E}$ is the vapour space expansion factor [-], and

$K_{S}$ is the vented vapour saturation factor [-].

The vapour expansion factor is determined by Equation B.19 for organic liquids with true vapour pressure less than $0.1 \mathrm{psi}$ and breather vents with \pm 0.03 psi relief pressure settings (EPA, 1995).

$$
K_{E}=0.001296\left(T_{A X}-T_{A N}\right)+0.000504 \cdot \alpha \cdot I
$$

where:

$T_{A X}$ is the daily maximum ambient temperature $\left[{ }^{\circ} \mathrm{R}\right]$,

$T_{A N}$ is the daily minimum ambient temperature $\left[{ }^{\circ} \mathrm{R}\right]$,

$\alpha$ is the tank paint solar absorptance [Btu/ $\mathrm{ft}^{2}$-day], and

$\mathrm{I}$ is the daily total solar insulation on the horizontal surface $\left[{ }^{\circ} \mathrm{R}-\mathrm{ft}^{2}\right.$-day/Btu].

For organic liquids with true vapour pressure above $0.1 \mathrm{psi}$, the relation presented in Eq. B.20, is used to calculate a more accurate estimate of the vapour expansion factor $\left(K_{E}\right)$. It should be noted that for any given input condition that results in a negative $K_{E}$ value, the standing losses are said to be zero (EPA, 1995). The uncertainties for Equation B.19 \& B.20 follow suit as Eq. B.3, where the bulk of uncertainties lay with the determination of vapour pressure for crude organic stocks that having varying compositions. For example, testing at a specific tank site presented by EPA (1995) found variations of $1.5 \mathrm{psig}$ in vapour pressure for the stored crude oil, which results in an approximate difference of $158 \mathrm{lb} /$ day in the calculated standing losses.

$$
K_{E}=\frac{\Delta T_{V}}{T_{L A}}+\frac{\Delta P_{V}-\Delta P_{B}}{P_{A}-P_{V A}}>0
$$

where:

$\Delta T_{V}$ is the daily vapour temperature range $\left[{ }^{\circ} \mathrm{R}\right]$,

$T_{L A}$ is the daily average liquid surface temperature [ $\left.{ }^{\circ} \mathrm{R}\right]$, 
$\Delta P_{V}$ is the vapour pressure range [psi],

$\Delta P_{B}$ is the breather vent pressure setting range [psi]; for typical \pm 0.03 vents is

$0.06 \mathrm{psi}$,

$P_{A}$ is the atmospheric pressure [psi], and

$P_{V A}$ is the vapour pressure at daily average liquid surface temperature [psi].

The vented vapour saturation factor $K_{S}$ is determined by the following relation (EPA, 1995).

$$
K s=\frac{1}{1+0.053 \cdot P_{V A} \cdot H_{V O}}
$$

where:

$P_{V A}$ is the vapour pressure at daily average liquid surface temperature [psi], and $H_{V O}$ is the vapour space outage height [ft].

The vapour space outage height is the measure of the equivalent internal height of the storage tank available for evaporation, which generally, is not a readily available quantity. EPA (1995) recommends that half the tank height be used to estimate the standing evaporative losses, however, it was found that $H_{V O}$ can be found to vary by \pm 15 $\mathrm{ft}$ (for a $40 \mathrm{ft}$ tank) which results in approximately $+150 \mathrm{lb} /$ day to $-40 \mathrm{lb} /$ day variations in the standing evaporative losses (Engineering Science, 1977).

\section{B.2.2 Working loses of Fixed Roof and Horizontal Tanks}

As stated previously, working losses for fixed roof and horizontal tanks occurs during the filling cycle of the tank, where the hydrocarbon saturated air vapour is displaced out of the storage tank by incoming organic liquid. The annular working losses for fixed roof and horizontal tanks can be estimated by Equation B.22 (EPA, 1995)

$$
L_{W}=N \cdot H_{L X} \cdot\left(\frac{\pi}{4}\right) \cdot D^{2} \cdot K_{N} \cdot K_{P} \cdot W_{V} \cdot K_{B}
$$

where:

$N$ is the number of turnovers per year $\left[\right.$ year $\left.^{-1}\right]$,

$H_{L X}$ is the maximum liquid height $\left[{ }^{\circ} \mathrm{R}\right]$, 
$D$ is the tank diameter [ft],

$K_{N}$ is the working loss turnover factor [-],

$$
\begin{aligned}
& \text { for } \mathrm{N}>36, K_{N}=(180+\mathrm{N}) / 6 \mathrm{~N} \\
& \text { for } \mathrm{N} \leq 36, K_{N}=1
\end{aligned}
$$

$K_{P}$ is the working loss product factor [-],

for crude oil, $K_{P}=0.75$

for all other organic liquids, $K_{P}=1$

$W_{V}$ is vapour density $\left[\mathrm{lb} / \mathrm{ft}^{3}\right]$, and

$K_{B}$ is the vent setting correction factor [-] ;

for open vents and typical pressure vents (up to $\pm 0.03 \mathrm{psi}$ ), $K_{B}=1$.

When the breather vent pressure relief settings are greater than typical values (of $\pm 0.03 \mathrm{psi}$ ) and the inequality (presented in Eq. B.23) is satisfied, then the vent correction factor $K_{B}$ can be determined by Eq. B.24. If the inequality cannot be satisfied, this implies the vent setting correction factor is 1 because the internal operating pressure is greater than the breather vent relief pressure setting, allowing for open vent losses. The uncertainties for the working losses are said to follow suit with the uncertainties of the vapour pressures (EPA 1995).

$$
\begin{array}{r}
K_{N}\left[\frac{P_{B P}+P_{A}}{P_{l}+P_{A}}\right]>1.0 \\
K_{B}=\left[\frac{\frac{P_{l}+P_{A}}{K_{N}}-P_{V A}}{P_{B P}+P_{A}-P_{V A}}\right]
\end{array}
$$

where:

$P_{l}$ is the actual gauge pressure of the vapour space [psi],

$P_{B P}$ is the breather vent pressure setting (upper limit) [psi],

$P_{A}$ is the atmospheric pressure [psi],

$P_{V A}$ is the vapour pressure at the daily average [psi], and

$K_{N}$ is the working loss turnover factor [-]; presented in Eq. B.22. 


\section{Appendix C Amplifier PCB Design}

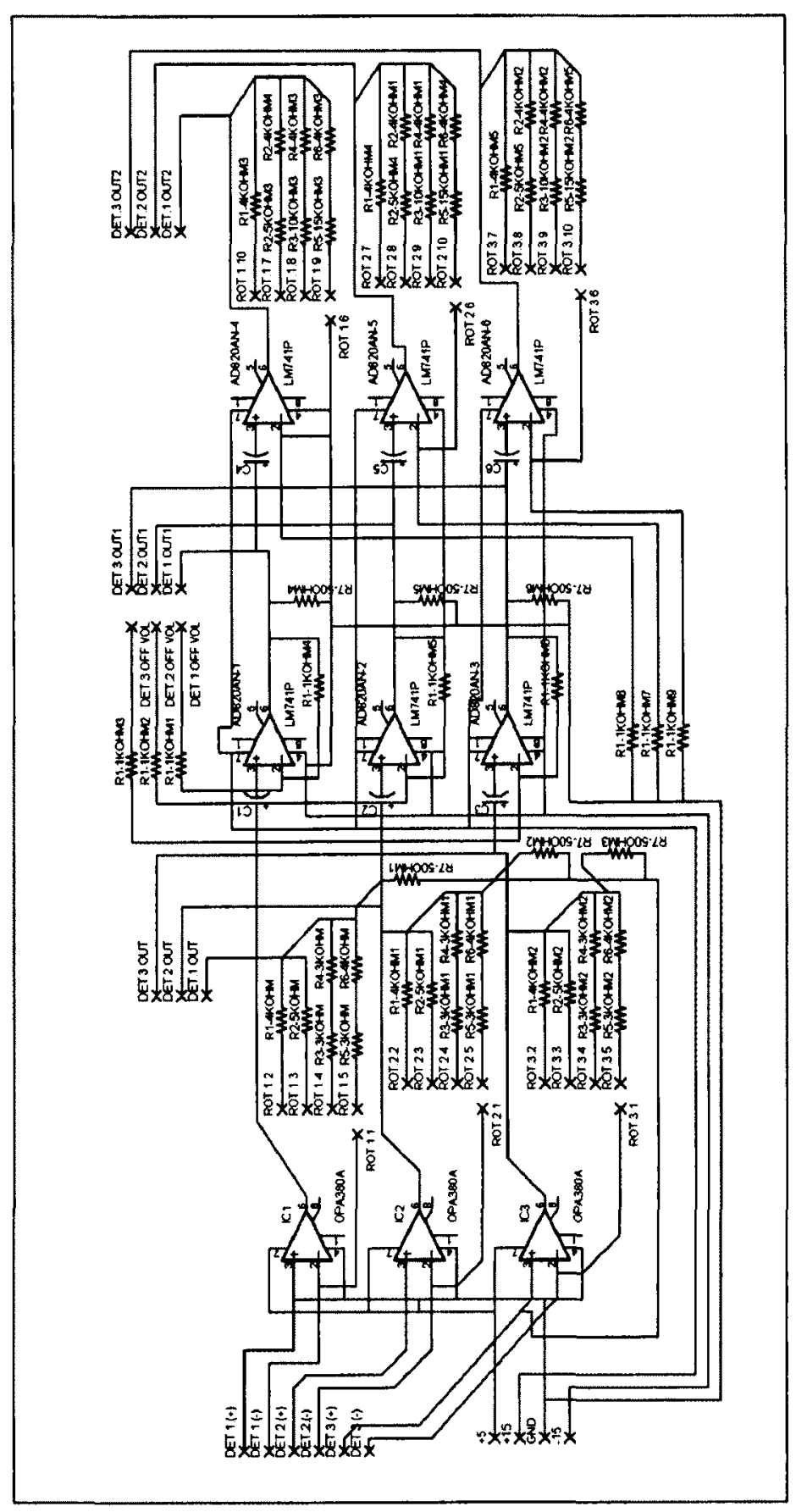

Figure C.1: PCB Schematic 


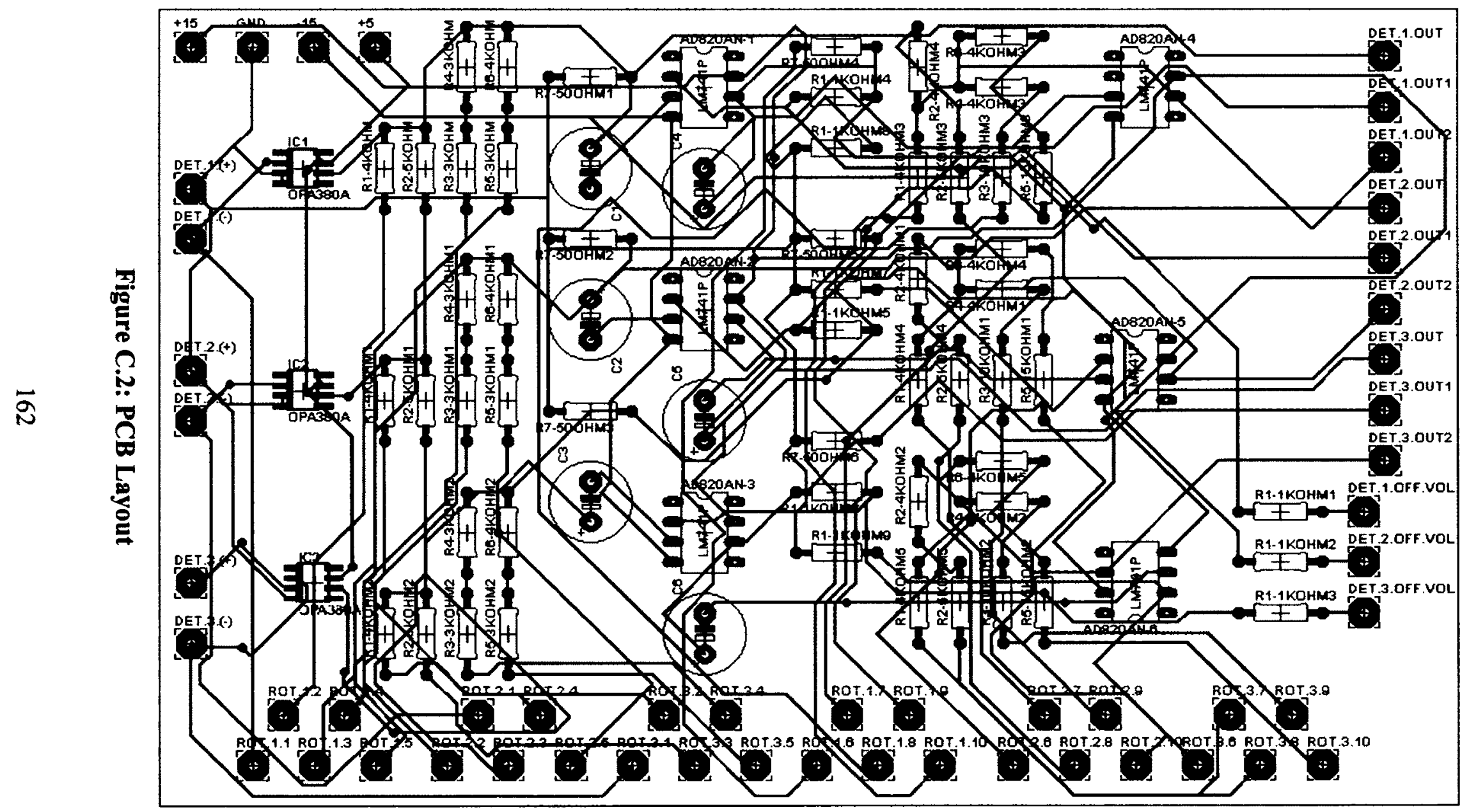




\section{Appendix D Beam Steering Tests}

In an optical system, beam steering occurs when light transverses a medium with a different refractive index than the surrounding gas or liquid. As indicated in the Schlieren imaging system, this phenomenon was utilized to visualize the hydrocarbon plume. However, if the hydrocarbon plume induces relatively large beam steering, this would result in power loss due to misalignment of the laser beam and the receiving optical components (i.e. focusing lens and mid-IR detector). Generally, beam steering due to density gradients is found to be very minimum as noted by Settlers (2001), but considering the large measurement path lengths of the full scale sensor $(\sim 90 \mathrm{~m}$ max $)$, further investigation was required.

To quantify this unknown value, an experiment was conducted using a visible

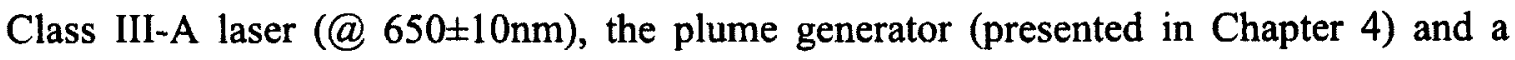
machine vision system (also presented in Chapter 4). The schematic of the experimental beam steering test apparatus is shown in Figure D.1 below.

Top View

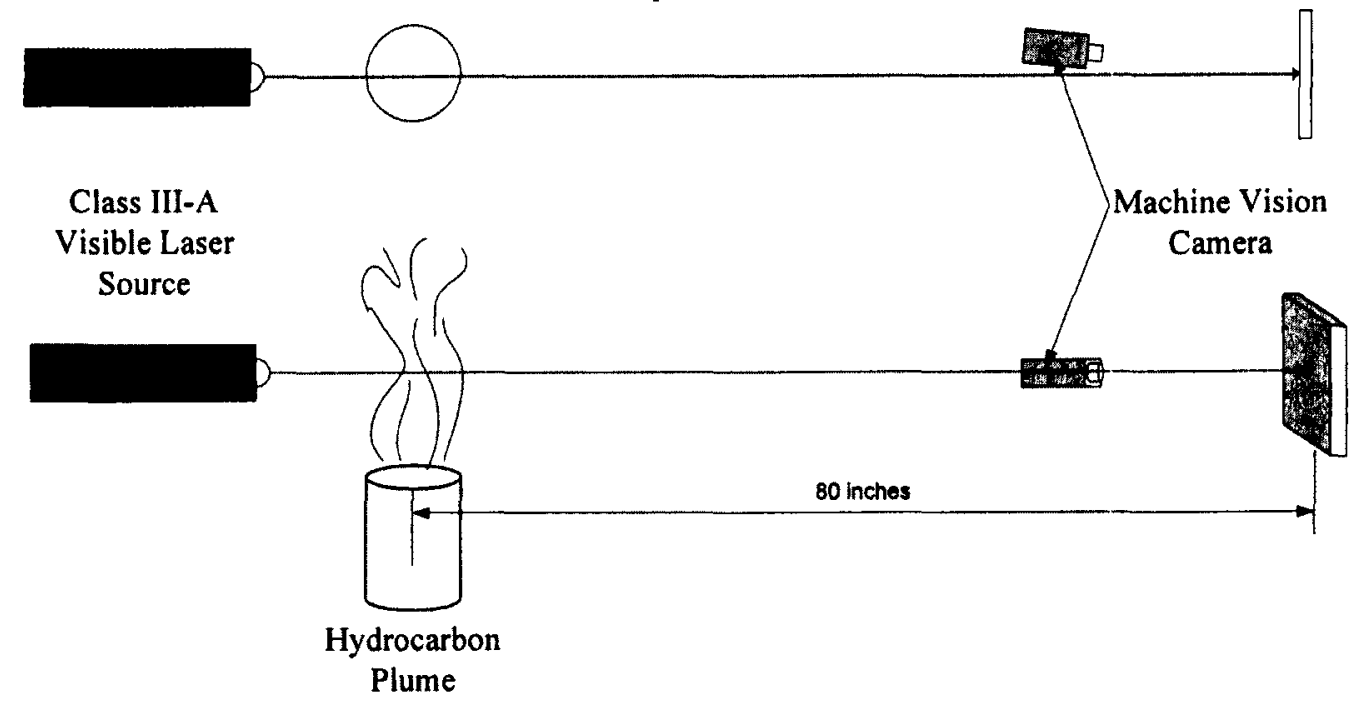

Figure D.1: Schematic of the beam steering experiment 
The visible laser was focused onto a calibration grid, which was placed 80 inches after the plume generator; a path length that was 3.5 times the in-lab VOC sensor. The 80 inch path length was selected because smaller distances yielded no measurable beam deviation using a machine vision camera.

The general methodology of the beam steering experiment was to acquire images (at $5 \mathrm{~Hz}$ ) of the visible laser termination (spot) on the $9 \mathrm{~mm} \times 9 \mathrm{~mm}$ grid board. Then using LabVIEW's image processing functions the centre of the laser spot was recorded and converted into real world coordinates using the pixel $/ \mathrm{mm}$ calibration factor of $0.257 \mathrm{~mm} / \mathrm{px}$ (taking in account the angle between the laser beam and the camera's optical axis). From the laser spot's coordinates, the radial distance from the "no plume" location was measured to quantify the displacement caused beam steering for a variety diluted hydrocarbon plumes. The experimental tests were conducted on hydrocarbon plumes of $0,0.15,0.3,0.45,0.6,0.75$, and 0.9 SLPM of methane in 3 SLPM of air, seen below.

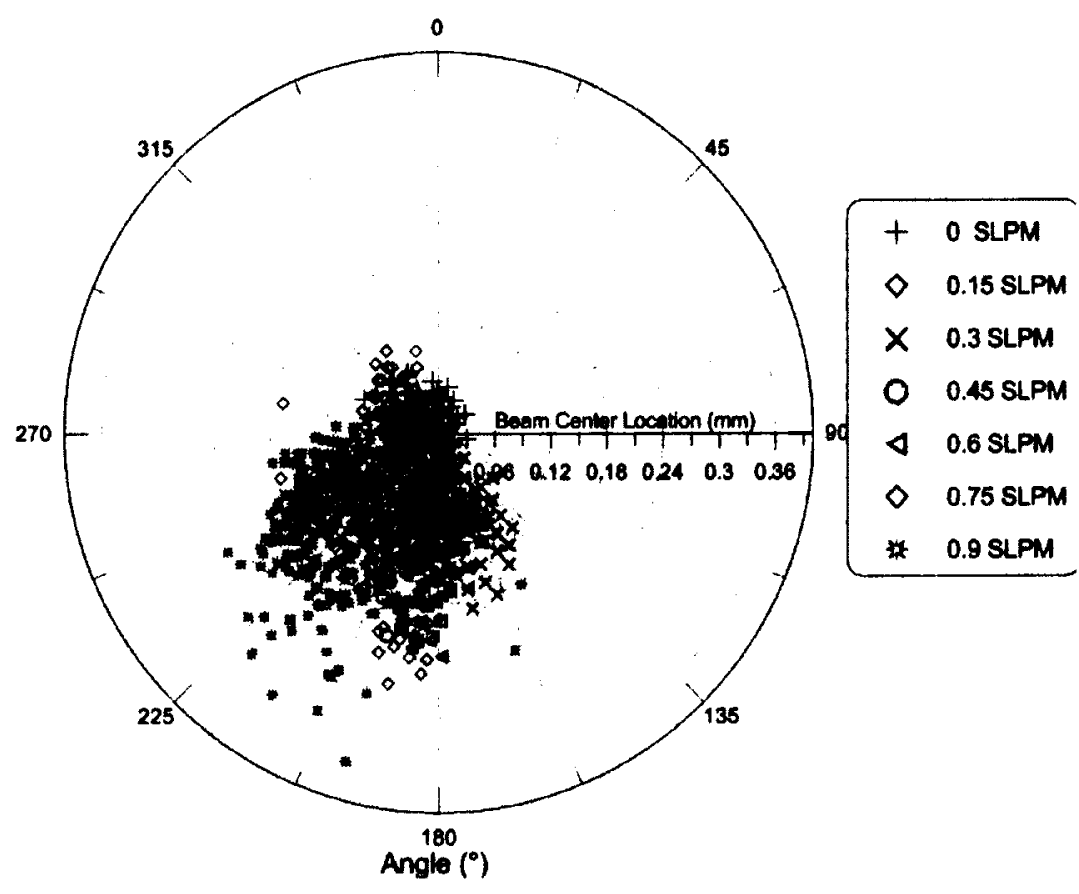

Figure D.2: Radial displacement of a visible laser traversing a hydrocarbon plume of 3.0 SLPM of air and $0,0.15,0.3,0.45,0.6,0.75$ and 0.9 SLPM of methane. 
As shown in Figure D.2, the average beam displacement measured shows minimal beam steering caused by the density gradient of the hydrocarbon plume. The highest methane set point shows that the average displacement is $0.135 \mathrm{~mm}$ for an 80 inch path length, yields a beam steering angle of $6.6781 \mathrm{e}-005$ radians $\left(0.0038^{\circ}\right)$. Assuming a $135 \mathrm{ppm}$ of methane over a $90 \mathrm{~m}$ path length (the maximum expected tank diameter), which is the equivalent path integrated concentration of the maximum experimental set point of $23.5 \%$ of methane over a $\sim 50.4 \mathrm{~mm}$ path length, the expected radial beam deflection is $\sim 6 \mathrm{~mm}$. Considering that the expected field concentration of methane is expected on the order of $3 \mathrm{ppm}$ level, it can be satisfactorily stated that beam steering should not have any adverse effects on the full scale VOC sensor, and the in-lab optical components (specifically, the $f=40 \mathrm{~mm} \mathrm{CaFl}_{2}$ focusing lens) are viable for the full scale sensor.

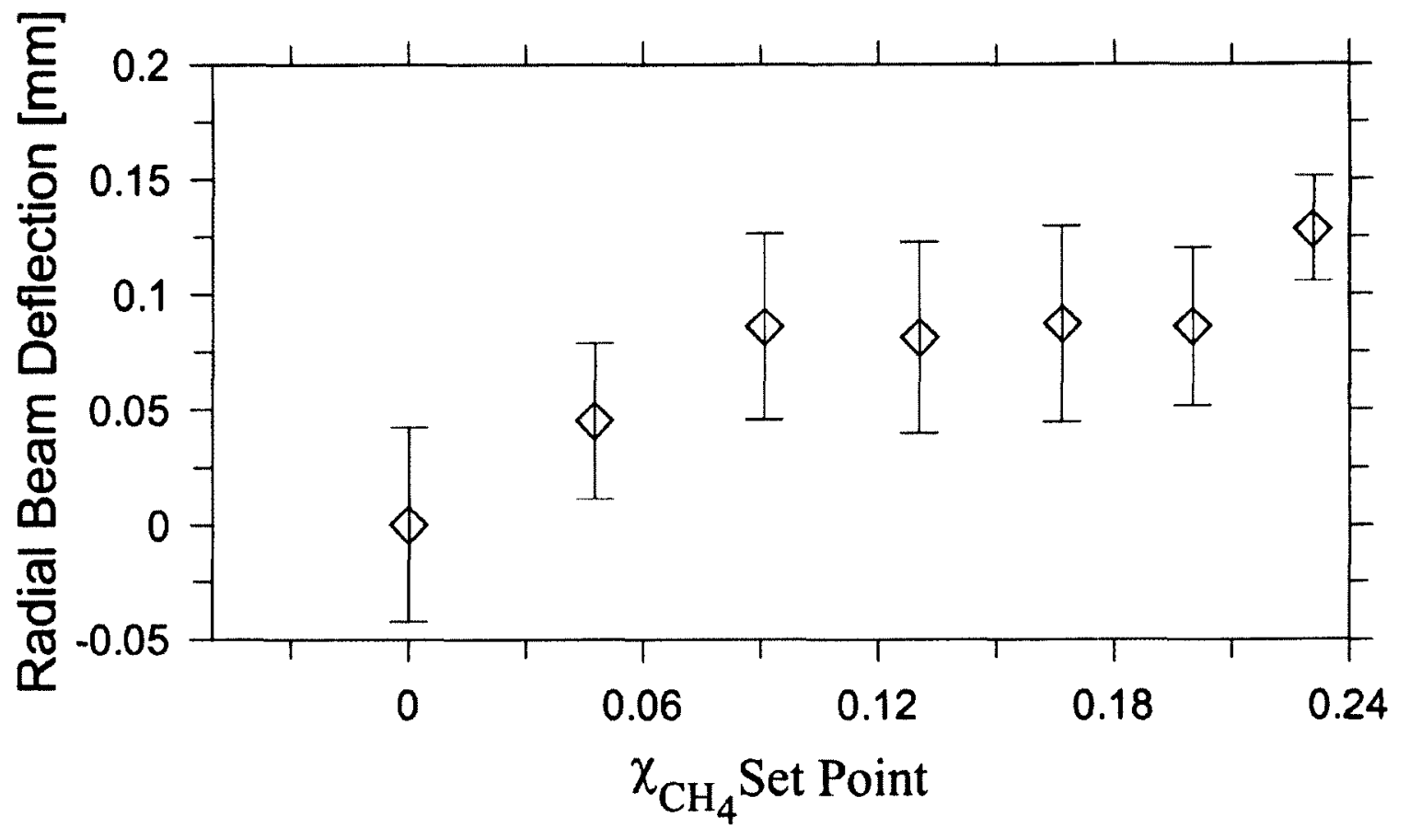

Figure D.3: Average radial beam deflection against the volumetric fraction of air$\mathrm{CH}_{4}$ plume. 